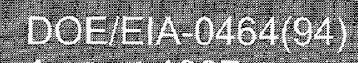

Augus 1907

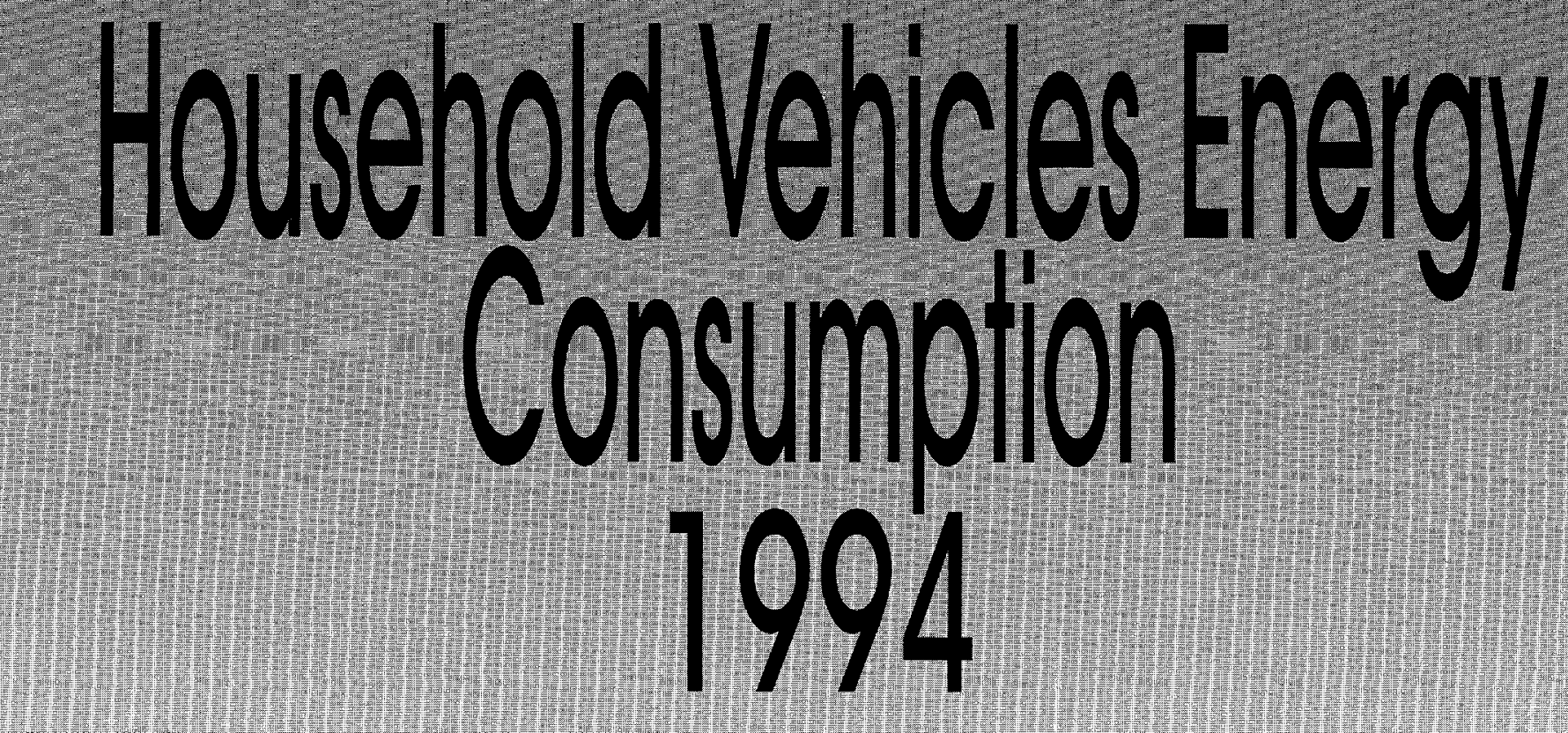

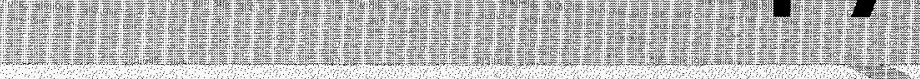

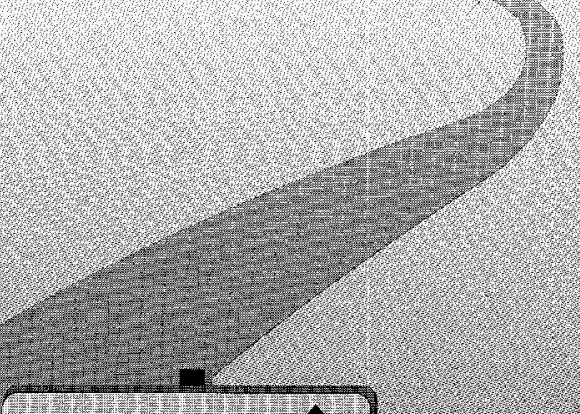

Information

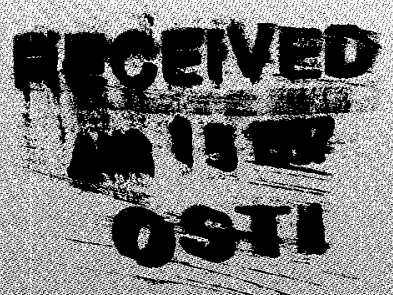
Highway

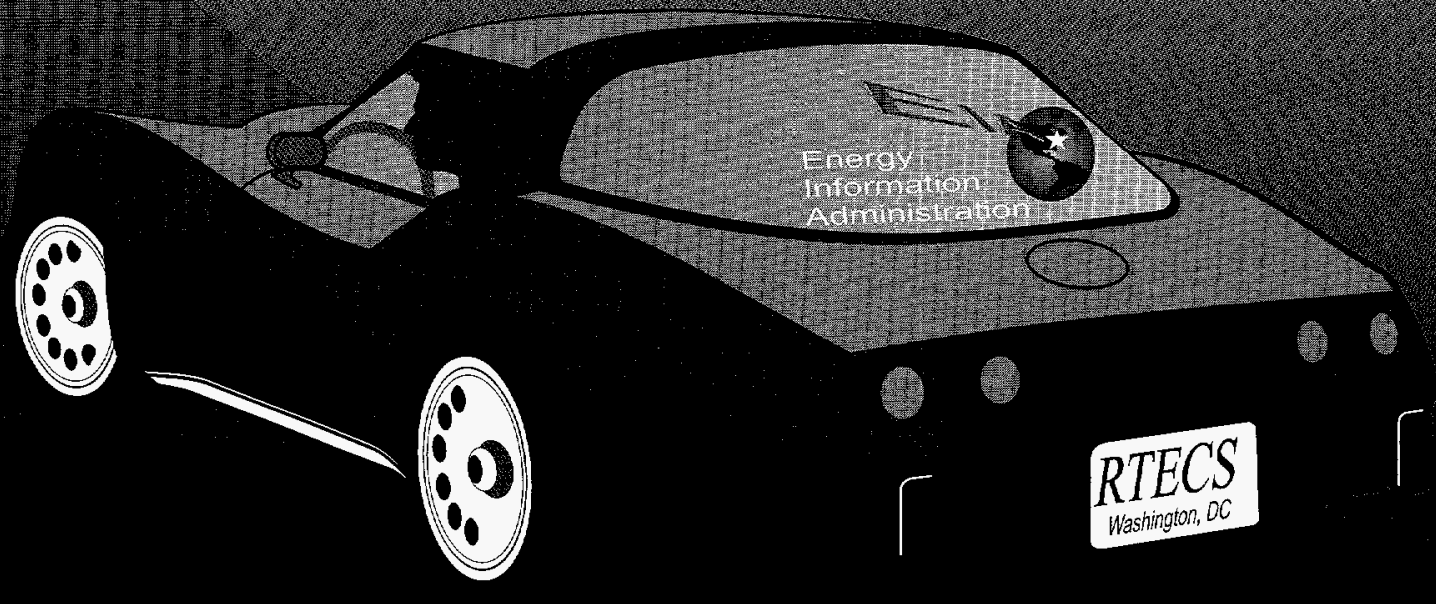




\section{HOW TO OBTAIN EIA PRODUCTS AND SERVICES}

For further information on any of the following services, or for answers to energy information questions, please contact EIA's National Energy Information Center:
National Energy Information Center (NEIC)
(202) 586-8800
Energy Information Administration
(202) 586-0727 (fax)
Forrestal Building, Room 1F-048
TTY: (202) 586-1181
Washington, DC 20585
E-mail: infoctr@eia.doe.gov

\section{Electronic Products and Services}

EIA's Internet Site Services offer nearly all EIA publications. Users can view and download selected pages or entire reports, search for information, download EIA data and analysis applications, and find out about new EIA information products and services.

World Wide Web: http://www.eia.doe.gov

Gopher: gopher://gopher.eia.doe.gov

FTP: ftp://ftp.eia.doe.gov

EIA also offers a listserve service for EIA press releases and other short documents. Sign up on the EIA World Wide Web site.

EIA's CD-ROM, Energy InfoDisc, contains most EIA publications, several databases, and an energy forecasting model. The Energy InfoDisc, produced quarterly, is available for a fee from STAT-USA, Department of Commerce, 1-800-STAT-USA.

The Comprehensive Oil and Gas Information Source (COGIS), a bulletin board service, contains data files from most of EIA's oil- and gas-related reports. It is available for a fee from STAT-USA, on 1-800-STAT-USA.

EIA's Electronic Publishing System (EPUB) bulletin board contains data files, directories, and forecasts from most EIA reports. It can be accessed free of charge by dialing (202) 586-2557.

Many of EIA's data files and modeling programs are available for sale on diskette, tape, or cartridge, through either the National Technical Information Service or the Office of Scientific and Technical Information, Department of Energy. Contact NEIC for information on specific products, sources, and media, and ordering instructions.

\section{Printed Publications}

EIA directories are available free of charge from NEIC. Recent EIA periodicals and other publications are available from the Government Printing Office. Older reports are available from the National Technical Information Service:

Superintendent of Documents

U.S. Government Printing Office

P.O. Box 371954

Pittsburgh, PA 15250-7954

(202) 512-1800; (202)-512-2250 (fax)
National Technical Information Service

U.S. Department of Commerce

5285 Port Royal Road

Springfield, VA 22161

(703) 487-4650; (703) 321-8547 (fax)

Released for Printing: July 24, 1997

Printed with soy ink on recycled paper

GPO Stock No: 061-003-01001-6 
DOE/EIA-0464(94)

Distribution Category UC-950

\title{
Household Vehicles Energy Consumption 1994
}

\author{
August 1997
}

\section{Energy Information Administration}

Office of Energy Markets and End Use

U.S. Department of Energy

Washington, DC 20585

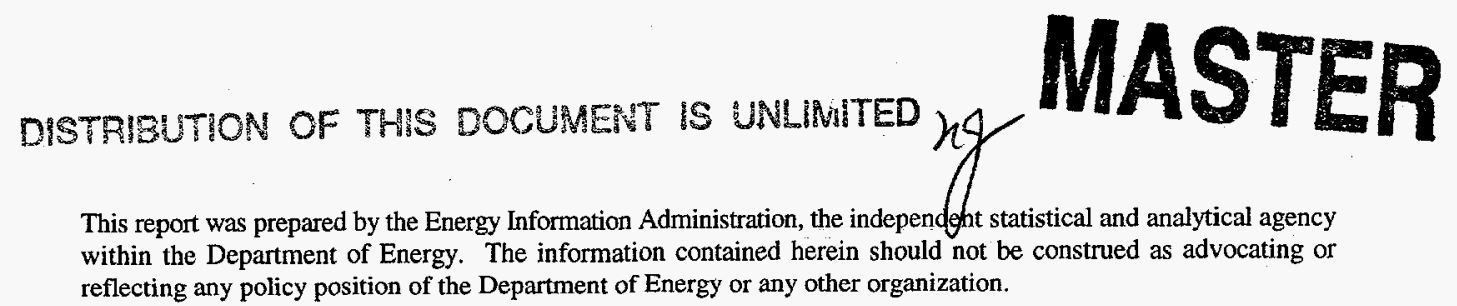





\section{DISCLAMIER}

Portions of this document may be illegible in electronic image products. Images are produced from the best available original document. 



\section{Contacts}

This publication was prepared by the Energy Information Administration under the general direction of W. Calvin Kilgore, Director of the Office of Energy Markets and End Use (202-586-1617). The project was directed by Dwight $K$. French, Chief of the Transportation and Industrial Branch in the Energy End Use and Integrated Statistics Division (EEUISD) (202-586-1126). Specific technical information may be obtained from the Residential Transportation Energy Consumption Survey (RTECS) Manager, Ron Lambrecht (202-586-4962). The fax number for all EEUISD personnel is $202-586-0018$.

Detailed technical questions on the topics indicated may be referred to the following members of the EEUISD:

\begin{tabular}{|c|c|c|c|}
\hline RTECS Manager & Ron Lambrecht & $202-586-4962$ & rlambrec@eia.doe.gov \\
\hline Vehicle Characteristics & $\begin{array}{l}\text { Ron Lambrecht } \\
\text { Barbara Fichman }\end{array}$ & $\begin{array}{l}202-586-4962 \\
202-586-5737\end{array}$ & $\begin{array}{l}\text { rlambrec@eia.doe.gov } \\
\text { bfichman@eia.doe.gov }\end{array}$ \\
\hline Vehicle-Miles Traveled & $\begin{array}{l}\text { Ron Lambrecht } \\
\text { Barbara Fichman }\end{array}$ & $\begin{array}{l}202-586-4962 \\
202-586-5737\end{array}$ & $\begin{array}{l}\text { rlambrec@eia.doe.gov } \\
\text { bfichman@eia.doe.gov }\end{array}$ \\
\hline $\begin{array}{l}\text { Fuel Economy, Consumption, } \\
\text { and Expenditures }\end{array}$ & $\begin{array}{l}\text { Ron Lambrecht } \\
\text { Barbara Fichman }\end{array}$ & $\begin{array}{l}202-586-4962 \\
202-586-5737\end{array}$ & $\begin{array}{l}\text { rlambrec@eia.doe.gov } \\
\text { bfichman@eia.doe.gov }\end{array}$ \\
\hline Report Editing and Production & $\begin{array}{l}\text { Ron Lambrecht } \\
\text { Barbara Fichman } \\
\text { Hattie Ramseur } \\
\text { Christy Hall }\end{array}$ & $\begin{array}{l}202-586-4962 \\
202-586-5737 \\
202-586-1124 \\
202-586-1068\end{array}$ & $\begin{array}{l}\text { rlambrec@eia.doe.gov } \\
\text { bfichman@eia.doe.gov } \\
\text { hramseur@eia.doe.gov } \\
\text { chall@eia.doe.gov }\end{array}$ \\
\hline $\begin{array}{l}\text { Public Use Data, Computer } \\
\text { Systems Design }\end{array}$ & Nanno Smith & $202-586-5841$ & nsmith@eia.doe.gov \\
\hline Detailed Statistical Tables & $\begin{array}{l}\text { Vicky Moorhead } \\
\text { Ron Lambrecht }\end{array}$ & $\begin{array}{l}202-586-1133 \\
202-586-4962\end{array}$ & $\begin{array}{l}\text { vmoorhea@eia.doe.gov } \\
\text { rlambrec@eia.doe.gov }\end{array}$ \\
\hline
\end{tabular}

EEUISD would like to thank Bethany Dickerson for verifying data in this report. 


\section{A Note to the Reader}

The Residential Transportation Energy Consumption Survey (RTECS) was fielded for the last time in 1994. This final RTECS report emphasizes changes in residential transportation from 1988, the earliest year for which consistent data are available, through 1994. During the 1988-to-1994 period, minivans and sport-utility vehicles became much more prevalent in the residential fleet and accounted for an increasing share of vehicle-miles traveled. The first section of this report illustrates those and other changes in residential transportation during the period. Chapters 1 through 4 explore trends in residential transportation and Chapter 5 presents detailed tables.

\section{For More Information}

As part of the Energy Information Administration's (EIA) mission to provide meaningful data from end-use energy surveys, EIA conducts ongoing user needs efforts to ascertain users' data requirements. Although the RTECS will not be fielded again, EIA is exploring alternative sources of residential vehicle data. If you have questions or suggestions about residential vehicle data, please contact Ron Lambrecht, RTECS Manager, at 202-586-4962 or at the address below.

If you have any data or report-related requirements or suggestions regarding any of the other ElA consumption surveys, please contact the appropriate survey manager directly, or use the address below.

Commercial Buildings Energy Consumption Survey (CBECS): Martha Johnson, Survey Manager, at 202-586-1135.

Manufacturing Energy Consumption Survey (MECS): Mark Schipper, Survey Manager, at 202-586-1136.

Residential Energy Consumption Survey (RECS): Robert Latta, Survey Manager, at 202-586-1385.

You are encouraged to provide your comments to the survey managers. Your feedback is important to us.

El-63, Mail Stop 2G-090

1000 Independence Avenue, SW

Washington, DC 20585

Fax: 202-586-0018 


\section{Contents}

Residential Transportation At a Glance, $1988-1994 \ldots \ldots \ldots \ldots \ldots \ldots \ldots \ldots \ldots \ldots \ldots \ldots \ldots$

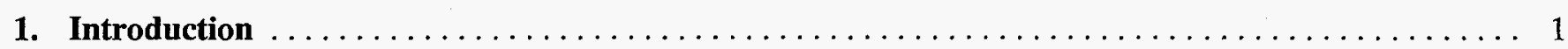

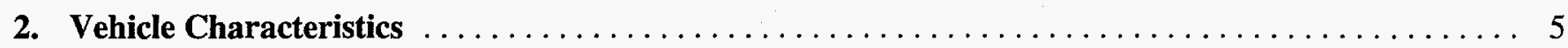

Changes in the Composition of the Residential Fleet $\ldots \ldots \ldots \ldots \ldots \ldots \ldots \ldots \ldots \ldots \ldots \ldots \ldots$

Residential Vehicles at the Household Level $\ldots \ldots \ldots \ldots \ldots \ldots \ldots \ldots \ldots \ldots \ldots \ldots \ldots \ldots \ldots \ldots$

Changes in Residential Vehicle Technology $\ldots \ldots \ldots \ldots \ldots \ldots \ldots \ldots \ldots \ldots \ldots \ldots \ldots \ldots \ldots \ldots$

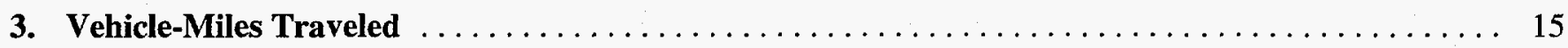

The Number of Vehicle-Miles Traveled Continued To Rise $\ldots \ldots \ldots \ldots \ldots \ldots \ldots \ldots \ldots \ldots \ldots \ldots \ldots$

Inside the Average U.S. Household: Who Drives the Most? $\ldots \ldots \ldots \ldots \ldots \ldots \ldots \ldots \ldots \ldots \ldots \ldots \ldots 18$

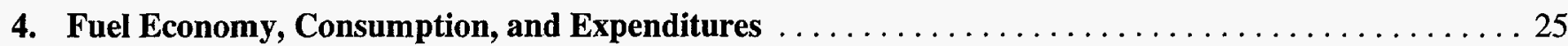

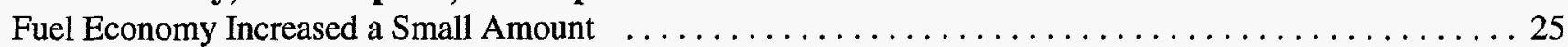

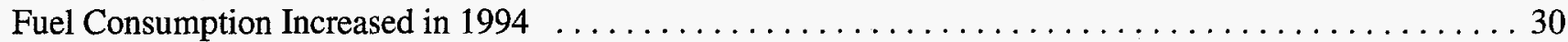

Fuel Expenditures Varied by Household Characteristics $\ldots \ldots \ldots \ldots \ldots \ldots \ldots \ldots \ldots \ldots \ldots \ldots \ldots \ldots$

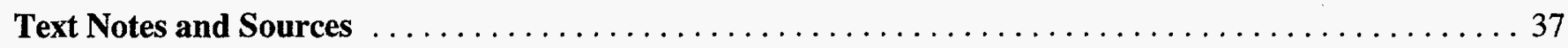

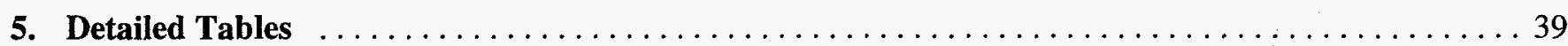

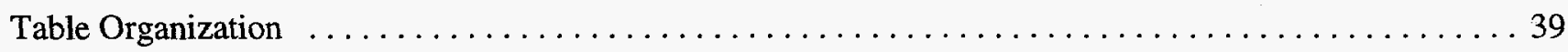

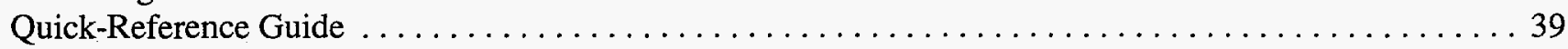

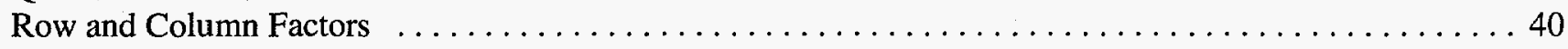

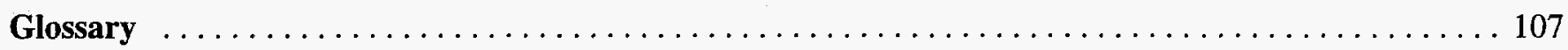




\section{Tables}

\section{Chapter 1}

1.1. Household Characteristics, Vehicle-Miles Traveled, Fuel Consumption, and Fuel Expenditures per Household, 1994 3

\section{Chapter 5}

5.1. U.S. Number of Vehicles, Vehicle Miles, Motor Fuel Consumption and Expenditures, $1994 \quad \ldots$. . . . 43

5.2. U.S. per Household Vehicle-Miles Traveled, Vehicle Fuel Consumption and Expenditures, $1994 \quad \ldots .47$

5.3. U.S. per Vehicle Miles Traveled, Vehicle Fuel Consumption and Expenditures, 1994 . . . . . . . . . 49

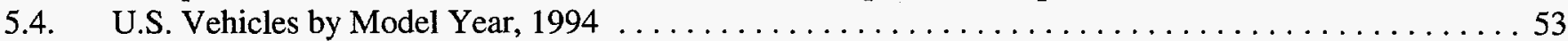

5.5. U.S. Vehicle Fuel Efficiency by Model Year, $1994 \ldots \ldots \ldots \ldots \ldots \ldots \ldots \ldots \ldots \ldots \ldots \ldots \ldots \ldots \ldots . \ldots$

5.6. U.S. Average Vehicle Fuel Consumption by Model Year, $1994 \ldots \ldots \ldots \ldots \ldots \ldots \ldots \ldots \ldots \ldots$

5.7. U.S. Vehicle-Miles Traveled by Family Income, $1994 \ldots \ldots \ldots \ldots \ldots \ldots \ldots \ldots \ldots \ldots \ldots \ldots \ldots$

5.8. U.S. Vehicle Fuel Consumption by Family Income, $1994 \ldots \ldots \ldots \ldots \ldots \ldots \ldots \ldots \ldots \ldots \ldots \ldots 6$

5.9. U.S. Average Vehicle-Miles Traveled by Family Income, $1994 \ldots \ldots \ldots \ldots \ldots \ldots \ldots \ldots \ldots$

5.10. U.S. Average Vehicle Fuel Consumption by Family Income, $1994 \ldots \ldots \ldots \ldots \ldots \ldots \ldots \ldots$

5.11. U.S. Vehicles by Household Composition, $1994 \ldots \ldots \ldots \ldots \ldots \ldots \ldots \ldots \ldots \ldots \ldots \ldots \ldots \ldots$

5.12. U.S. Average Vehicle-Miles Traveled by Household Composition, $1994 \ldots \ldots \ldots \ldots \ldots \ldots$. . . . . . 81

5.13. U.S. Average Vehicle-Miles Traveled by Vehicle Fuel Efficiency Category, $1994 \ldots \ldots \ldots \ldots$. . . . . 83

5.14. U.S. Vehicle Fuel Consumption by Vehicle Type, $1994 \ldots \ldots \ldots \ldots \ldots \ldots \ldots \ldots \ldots \ldots \ldots \ldots \ldots 7$

5.15. U.S. Average Vehicle-Miles Traveled by Vehicle Type, $1994 \ldots \ldots \ldots \ldots \ldots \ldots \ldots \ldots \ldots \ldots \ldots$

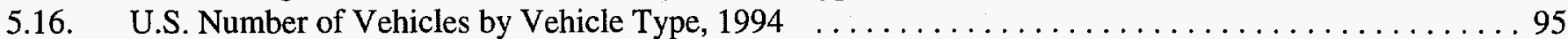

5.17. U.S. Number of Households by Vehicle Fuel Expenditures, 1994 . . . . . . . . . . . . . . . . . . . . . 99

5.18. U.S. Average Household and Vehicle Energy Expenditures, $1994 \ldots \ldots \ldots \ldots$. . . . . . . . . . . . . 102 


\section{Figures}

\section{Chapter 2}

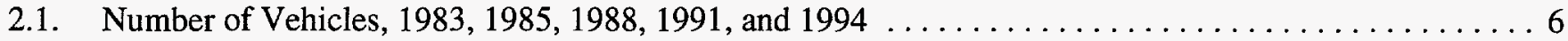

2.2. Number of Residential Vehicles by Type, 1988 and $1994 \ldots \ldots \ldots \ldots \ldots \ldots \ldots \ldots \ldots \ldots \ldots \ldots$

2.3. Change in Number of Residential Vehicles by Type, 1988 to $1994 \ldots \ldots \ldots \ldots \ldots \ldots \ldots \ldots \ldots$. . . . . . 7

2.4. Sales of New Passenger Cars and New Light Trucks for Model Years $1980-1995 \ldots \ldots \ldots$. . . . . . . 8

2.5. Number of Pre-1983 Residential Vehicles by Type, 1988, 1991, and $1994 \ldots \ldots \ldots \ldots \ldots \ldots \ldots$

2.6. Light Trucks' Share of Each Region's Residential Fleet, $1994 \ldots \ldots \ldots \ldots \ldots \ldots \ldots \ldots \ldots \ldots \ldots$

2.7. Average Engine Size for Residential Vehicles for Model Years 1980 - 1995 . . . . . . . . . . . . . . 12

2.8. Number of Cylinders in Residential Vehicle Engines, 1988 and $1994 \ldots \ldots \ldots \ldots \ldots \ldots \ldots \ldots \ldots$

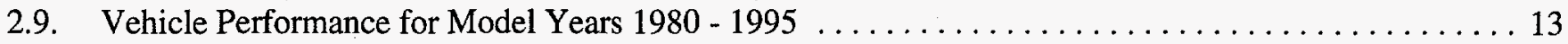

\section{Chapter 3}

3.1. Residential Vehicle-Miles Traveled by Type of Vehicle, 1988, 1991, and $1994 \ldots \ldots \ldots \ldots$

3.2. Residential Vehicle-Miles Traveled per Household and per Vehicle by Survey Year,

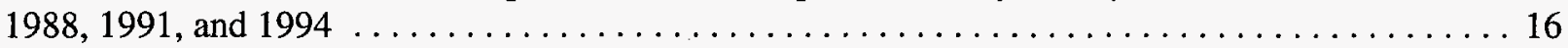

3.3. Residential Vehicle-Miles Traveled per Vehicle by Region, $1994 \ldots \ldots \ldots \ldots \ldots \ldots \ldots$

3.4. Residential Vehicle-Miles Traveled per Vehicle by Vehicle Age, 1994 . . . . . . . . . . . . . . . . 19

3.5. Residential Vehicle-Miles Traveled per Household by Household Size and Survey Year, 1988 and

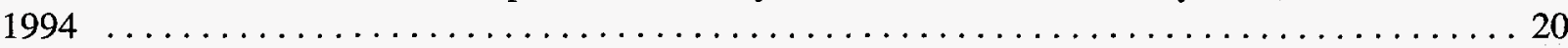

3.6. Residential Vehicle-Miles Traveled per Household by Number of Drivers and Survey Year, 1988 and 1994

3.7. Residential Vehicle-Miles Traveled per Household by Household Composition and Survey Year, 1988
and $1994 \ldots \ldots \ldots \ldots \ldots \ldots \ldots \ldots \ldots \ldots \ldots \ldots \ldots \ldots \ldots \ldots \ldots \ldots \ldots \ldots \ldots \ldots \ldots \ldots \ldots \ldots \ldots \ldots \ldots$
3.8. Residential Vehicle-Miles Traveled per Vehicle by Age of Primary Driver, $1994 \ldots \ldots \ldots \ldots \ldots \ldots$

3.7. Residential Vehicle-Miles Traveled per Household by Household Composition and Survey Year, 1988
and $1994 \ldots \ldots \ldots \ldots \ldots \ldots \ldots \ldots \ldots \ldots \ldots \ldots \ldots \ldots \ldots \ldots \ldots \ldots \ldots \ldots \ldots \ldots \ldots \ldots \ldots \ldots \ldots \ldots$
3.8. Residential Vehicle-Miles Traveled per Vehicle by Age of Primary Driver, $1994 \ldots \ldots \ldots \ldots \ldots \ldots$

3.9. Residential Vehicle-Miles Traveled per Household by Annual Household Income, $1994 \ldots \ldots \ldots \ldots 23$

3.10. Average Number of Vehicles per Household by Household Income, $1994 \ldots \ldots \ldots \ldots \ldots \ldots$

\section{Chapter 4}

4.1. Average Fuel Economy of Residential Vehicles for Model Years Through 1995 . . . . . . . . . . . . 26

4.2. Average Fuel Economy of Residential Vehicles by Type of Vehicle, 1988 and 1994 . . . . . . . . . . 27

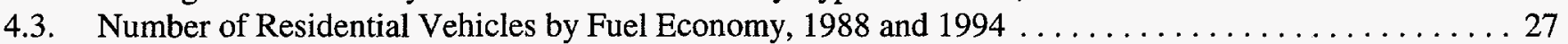

4.4. Average Fuel Economy of Residential Vehicles by Household Composition, $1994 \ldots \ldots \ldots$. . . . . . 29

4.5. Average Fuel Economy of Residential Vehicles by Annual Household Income, 1994 . . . . . . . . . . 29

4.6. Total Residential Vehicle Fuel Consumption, 1983, 1985, 1988, 1991, and $1994 \ldots \ldots \ldots \ldots . \ldots 30$

4.7. Total Residential Vehicle Fuel Consumption by Type of Vehicle, 1988 and $1994 \ldots \ldots \ldots \ldots$. . . . . 31

4.8. Average Residential Vehicle Fuel Consumption by Type of Vehicle, 1988 and $1994 \ldots \ldots \ldots \ldots 32$

4.9. Average Residential Vehicle Fuel Consumption per Vehicle for Model Years Through $1995 \ldots \ldots$. . 32

4.10. Average Annual Residential Vehicle Fuel Consumption by Region, $1994 \ldots \ldots \ldots \ldots \ldots \ldots$

4.11. Average Fuel Expenditures per Residential Vehicle by Survey Year, 1980, 1983,

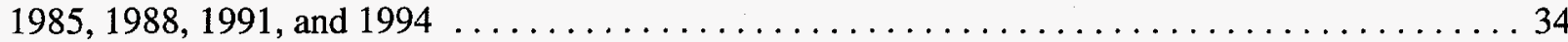

4.12. Average Vehicle Fuel Expenditures per Household, $1994 \ldots \ldots \ldots \ldots \ldots \ldots \ldots \ldots \ldots \ldots \ldots \ldots$

4.13. Average Vehicle Fuel Expenditures and Household Energy Expenditures by Household Income, 199435 



\section{Information}

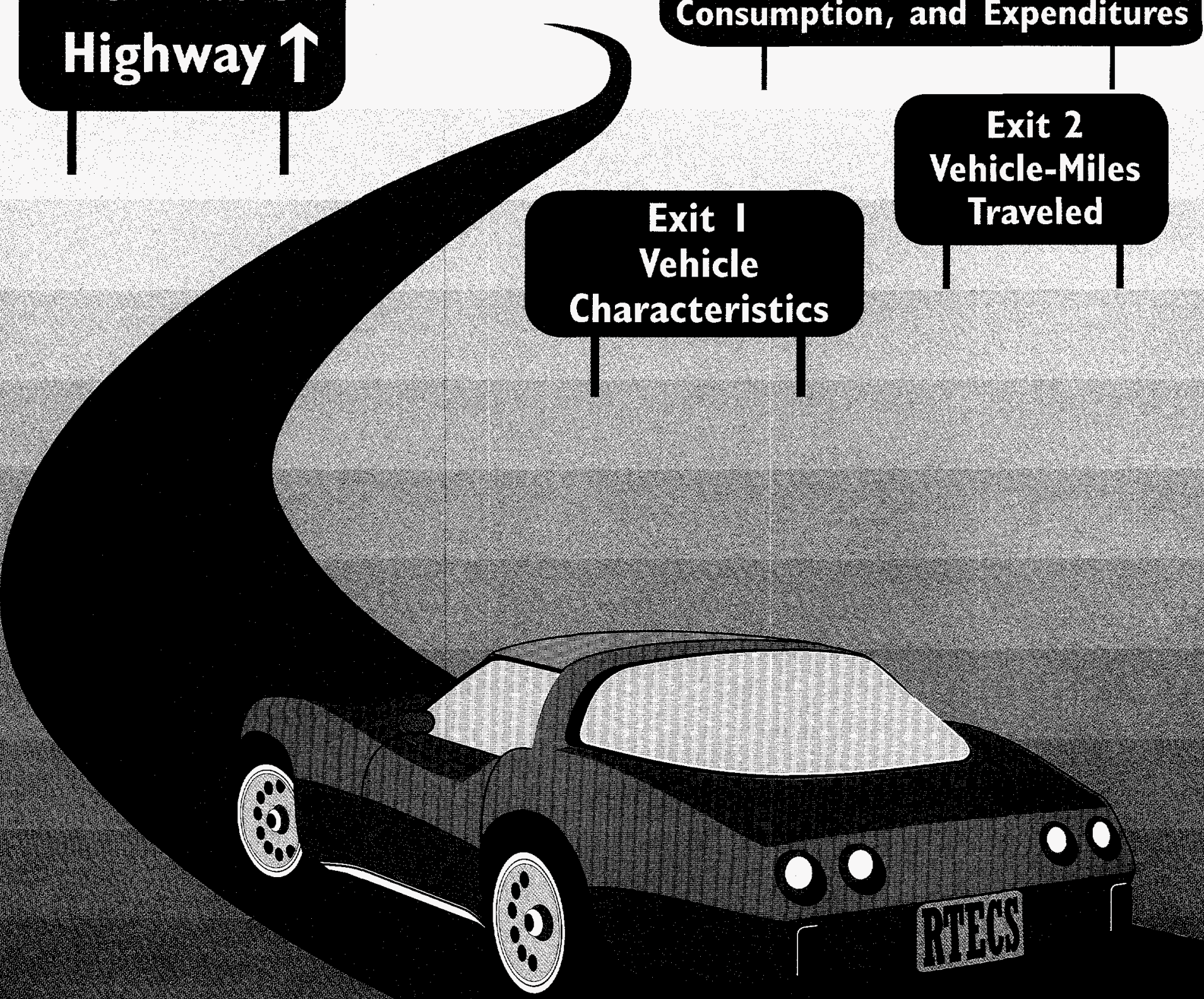

Household Vehicles Energy Consumption 1994 presents statistics about energy-related characteristics of highway vehicles available for personal use by members of U.S. households. The data were collected in the 1994 Residential Transportation Energy Consumption Survey, the final cycle in a series of nationwide energy consumption surveys conducted during the 1980 's and 1990's by the Energy Information Administration. 
Minivans and Sport-Utility Vehieles Becane Vore Popular.

Percent Distribution of Total

Residential Vehicle Fleet by

Vehicle Type, 1988 and 1994

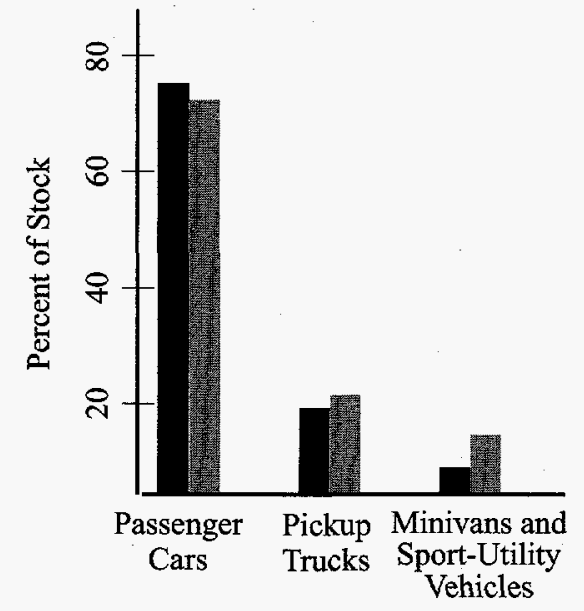

Percent Distribution of Newer Vehicles ( 2 model years old or less) by Vehicle Type, 1988 and 1994

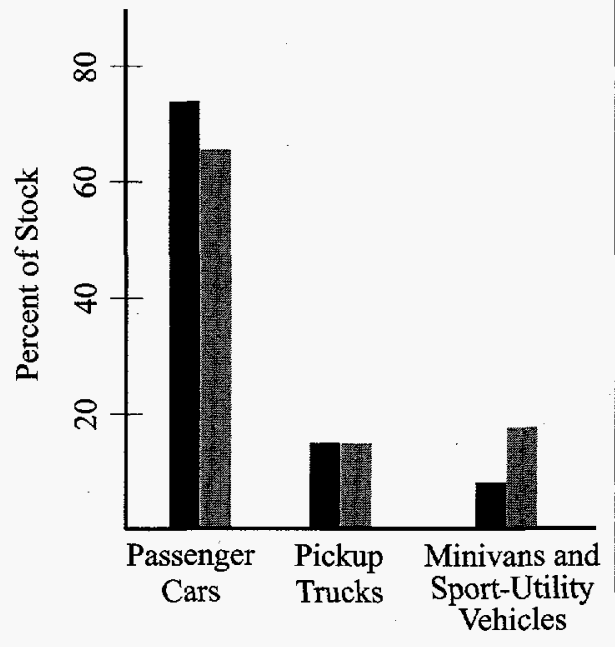

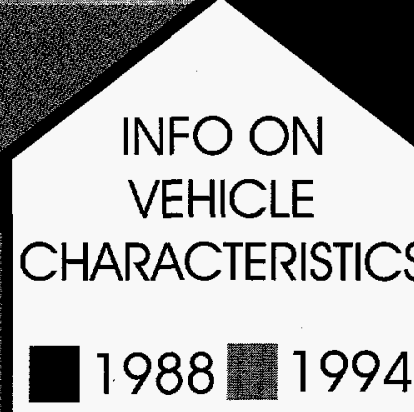
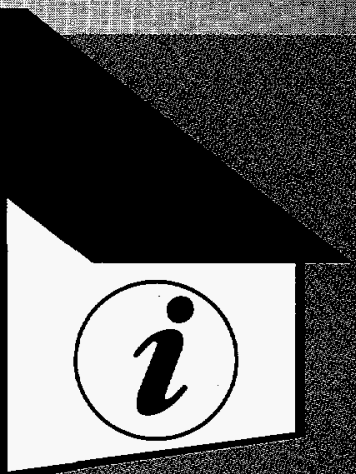


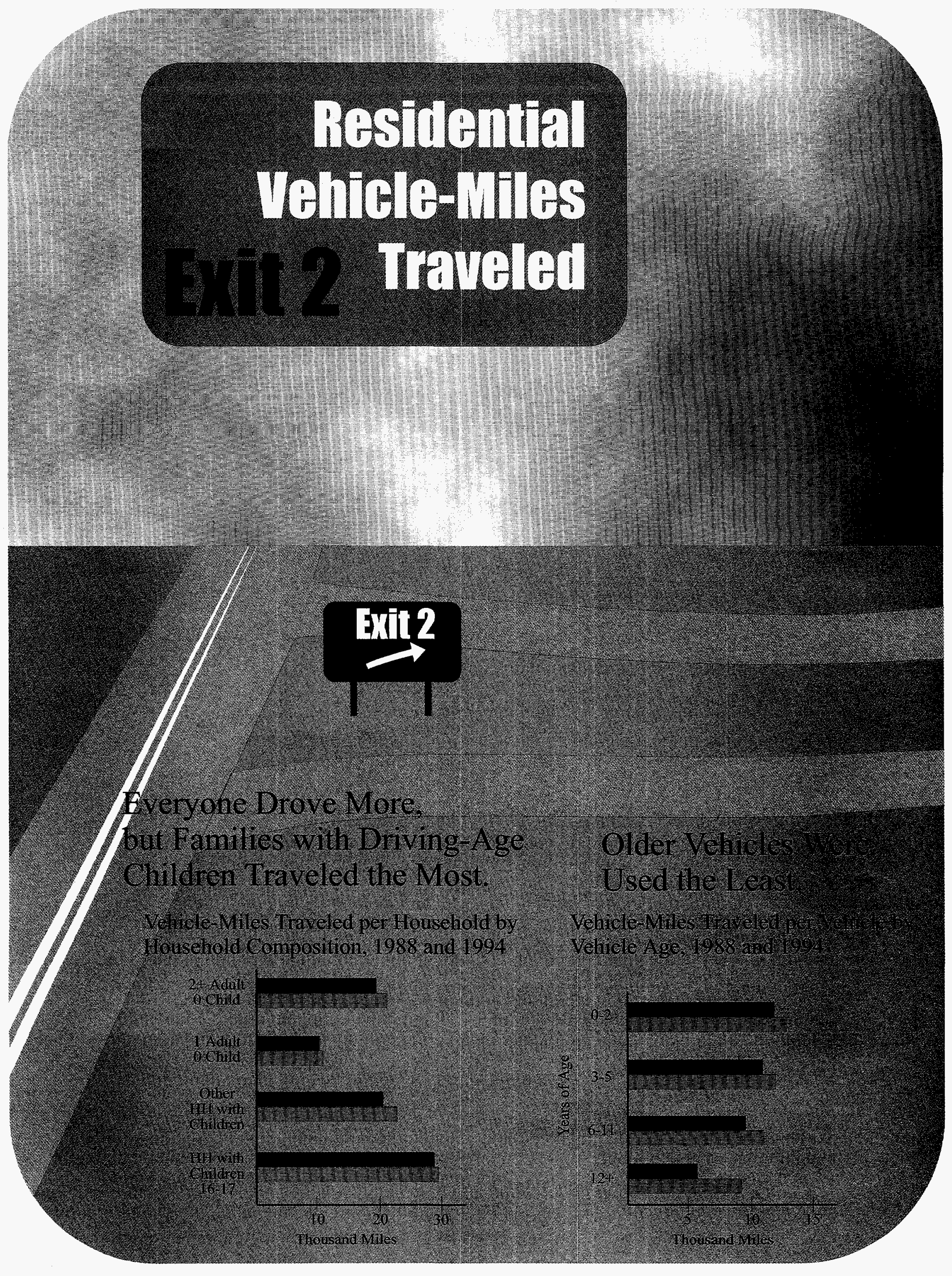


Vehicles per Household Remained Steady, While Driving Increased

Vehicles per Household and Vehicle-Miles

Traveled per Household, 1988 and 1994

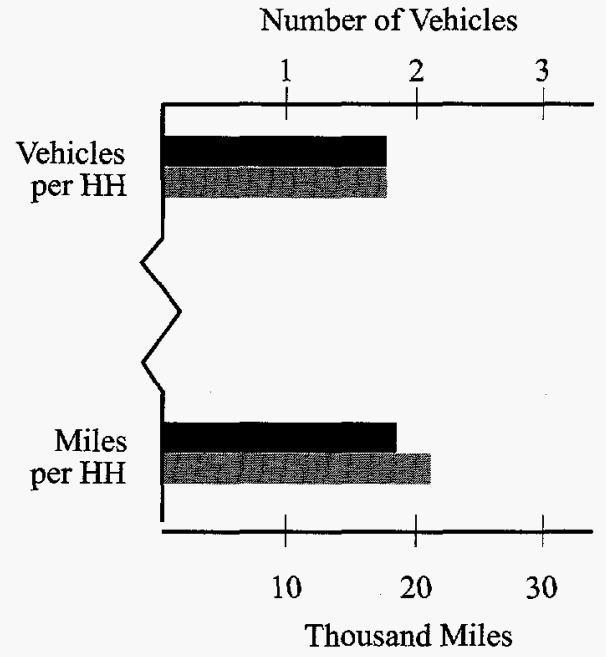

\section{Especially Outside}

\section{of the Suburibs.}

Vehicle-Miles Traveled per Household by Urban Status, 1988 and 1994

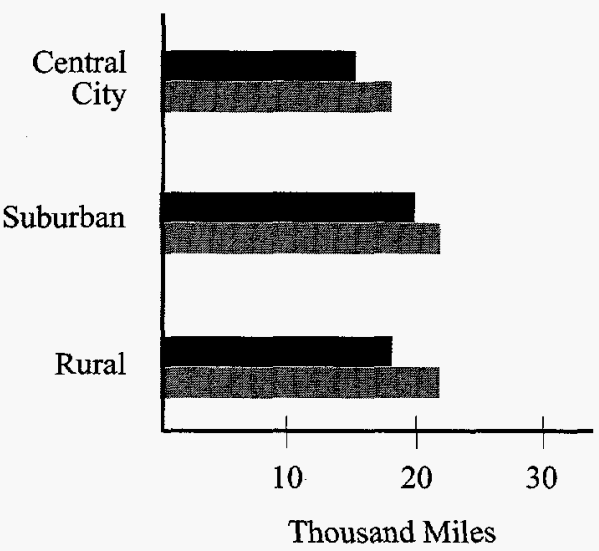

\section{Households in the "Wide-Open West". But Higher-Inconne}

Did Not Drive More Than Others . Howseholds Did.

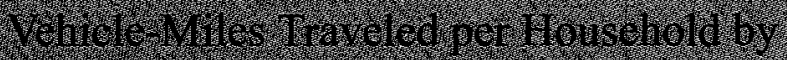

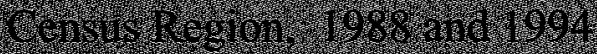

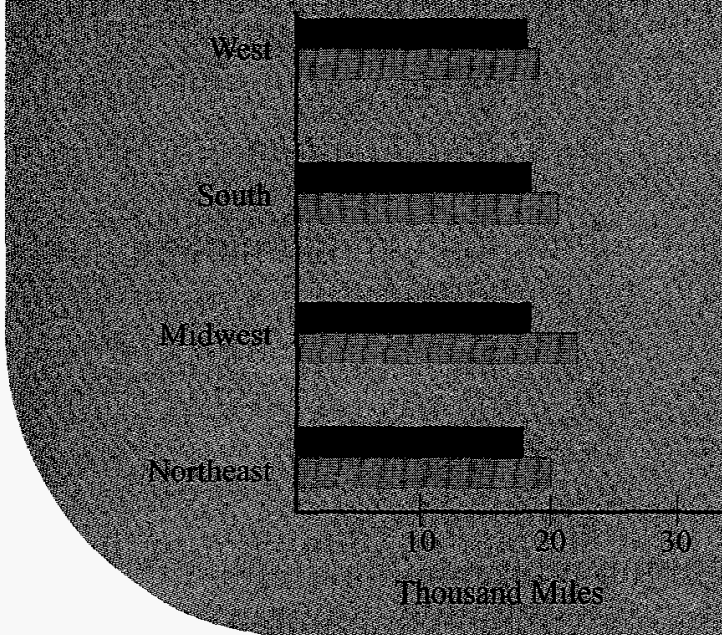

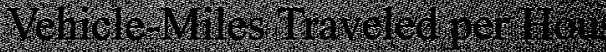

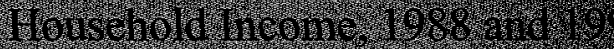




\section{Besidential Vohiche Fucl Economy, Bonsinmption, and Expendilities}

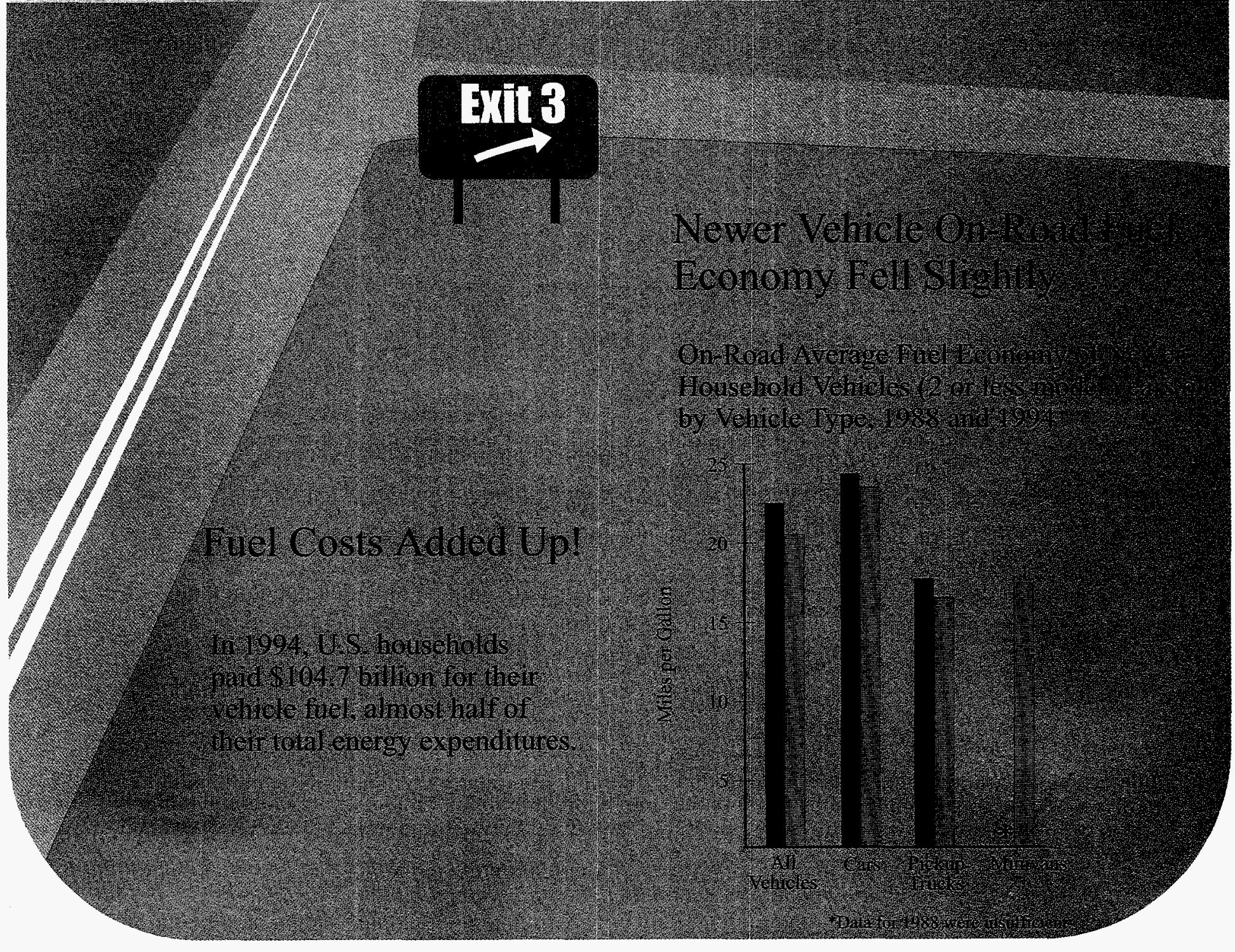


Uouseholds Paid More For Vehicle Fuel in 1994 than They Did in 1988.

Final Average Expenditures per Household for Vehicle Fuel, 1988 and 1994

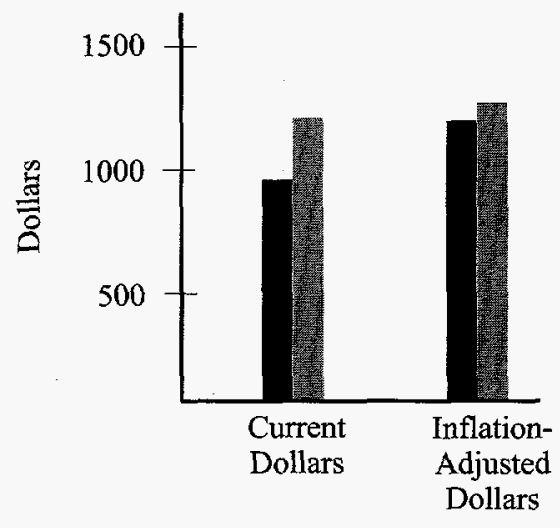

\section{But the Fuel Econony of the Total} Fleet Contimued To Rise Slowly:

On-Road Average Tiel Ecoinomy of Househohd Vehiche Fleet by Vehicle type, 1988 and 1994
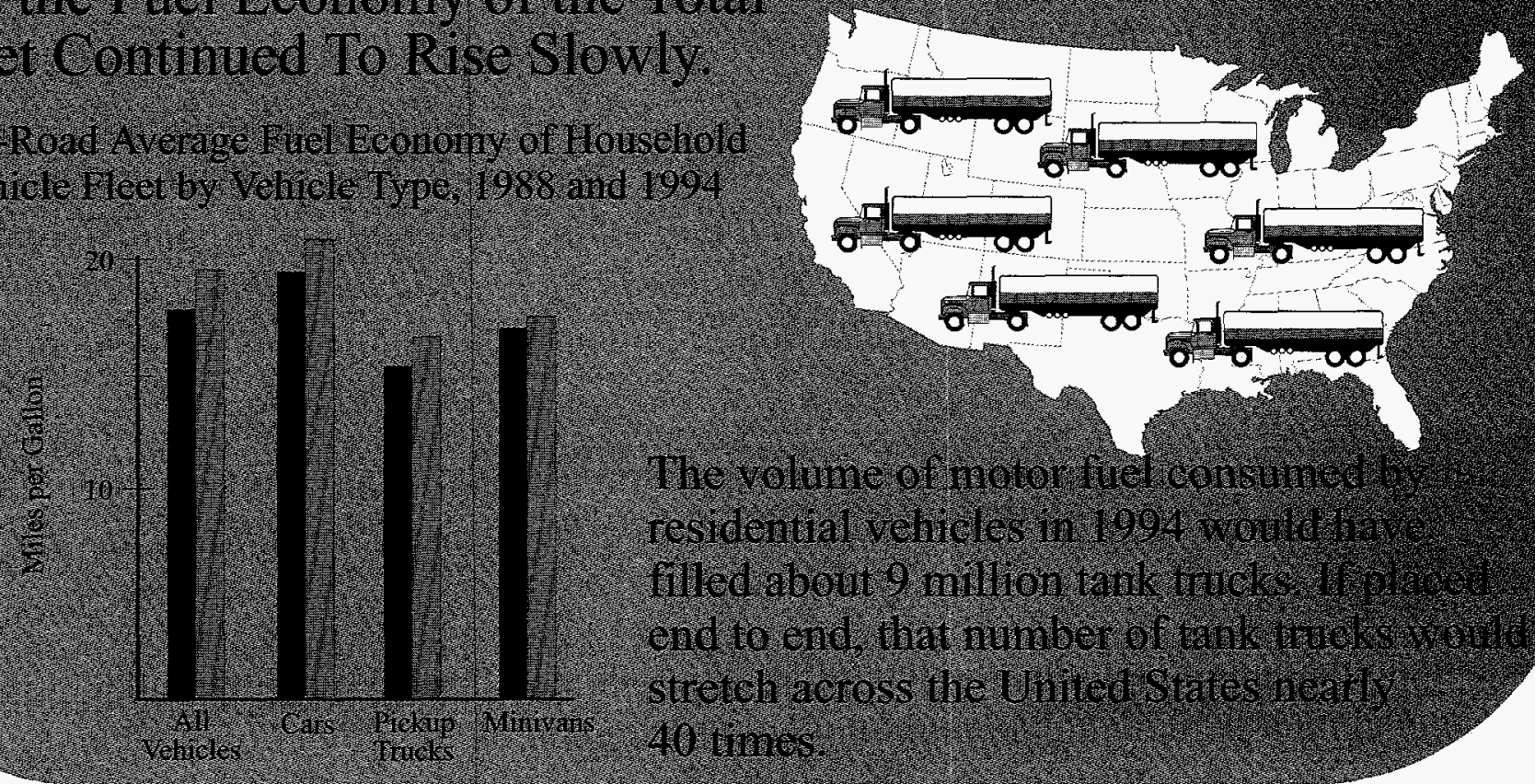

The volume of motor fiel consumer

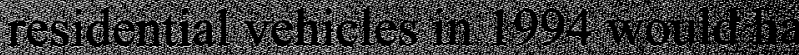
filled sbout 9 million tank tircl. end to end that number of rank ituek stretch across the United states nearly 40. imines: 


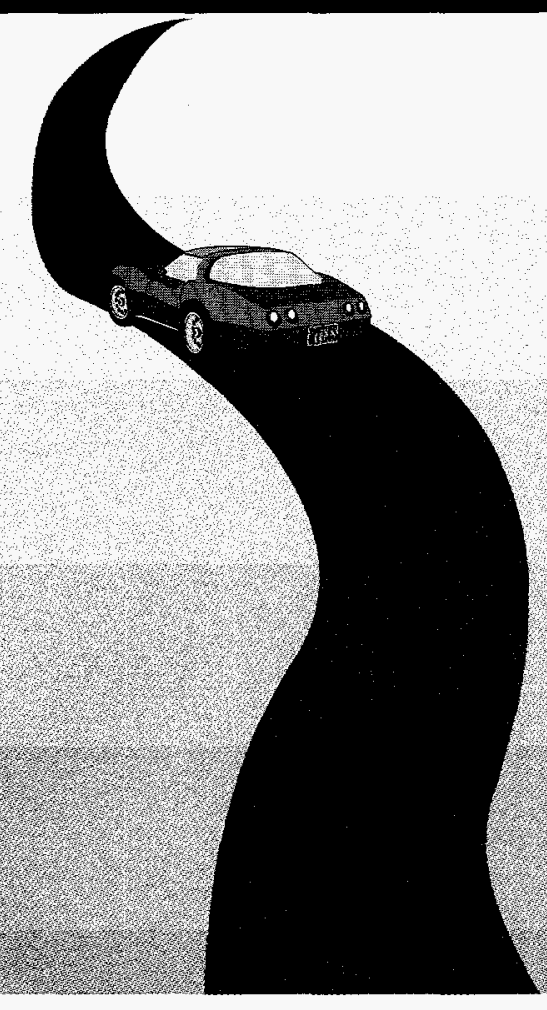

From 1988

Higher nominal prices per gallon,

$\$ 0.984$ versus

$\$ 1.156$

led to higher nominal expenditures per vehicle,

$\$ 550$

$\$ 998$

and per household,

1.8 vehicles

10.2 thousand

$18.3 \mathrm{mpg}$

559 gallons

$$
\text { versus }
$$

$\$ 668$,

$\$ 1,234$, and fuel economy improved from

The total number of residential vehicles increased from

to

156.8 million,

while the number of vehicles per vehicle-operating household remained constant at

and

1.8 vehicles.

Average miles traveled per vehicle jumped from

to

11.4 thousand,

to

$19.8 \mathrm{mpg}$,

so that fuel use per vehicle remained fairly constant at and but, when adjusted for inflation, expenditures changed little:

$\$ 1,218$ versus

$\$ 1,234$.

Together with the ongoing growth of the country, these trends caused the higher national demand for residential vehicle fuel to rise from
82.4 billion gallons
to
90.6 billion gallons,

and corresponding fuel costs to rise substantially, from

\$81.1 billion to

\$104.7 billion,

although the inflation-adjusted total cost was 


\section{Chapter 1. Introduction}

Household Vehicles Energy Consumption 1994 reports on the results of the 1994 Residential Transportation Energy Consumption Survey (RTECS). The RTECS is a national sample survey that has been conducted every 3 years since 1985. For the 1994 survey, more than 3,000 households that own or use some 6,000 vehicles provided information to describe vehicle stock, vehicle-miles traveled, energy end-use consumption, and energy expenditures for personal vehicles. The survey results represent the characteristics of the 84.9 million households that used or had access to vehicles in 1994 nationwide. (An additional 12 million households neither owned nor had access to vehicles during the survey year.) To be included in the RTECS survey, vehicles must be either owned or used by household members on a regular basis for personal transportation, or owned by a company rather than a household, but kept at home, regularly available for the use of household members. Most vehicles included in the RTECS are classified as "lightduty vehicles" (weighing less than 8,500 pounds). However, the RTECS also includes a very small number of "other" vehicles, such as motor homes and larger trucks that are available for personal use.

\section{What Are the Major Findings of the Survey?}

Members of U.S. households drove more miles and consumed more fuel in 1994 than in 1988 . Vehicle-miles traveled per household increased by 13 percent and the average fuel consumption per household increased by 5 percent. Household expenditures for motor gasoline, when adjusted for inflation, rose by 6 percent between 1988 and 1994. Fuel economy, as measured in miles per gallon (mpg), increased by 8 percent over the 6 -year period.

The average number of vehicles per household remained constant between 1988 and 1994 at approximately 1.8 , while the number of households with vehicles reported in the 1988 survey was 81.3 million, compared with 84.9 million in 1994. The total number of vehicles nationwide rose from 148 million to 157 million, an increase of 6 percent.

The composition of the vehicle stock continued its shift away from passenger cars toward minivans and sport-utility vehicles. In 1988, passenger cars comprised nearly three-quarters of the total vehicle stock, but by 1994, that share had dropped to 68 percent. The number of minivans and sport-utility vehicles grew by 42 percent, increasing their share from 5 percent of the total vehicle stock in 1988 to 11 percent in 1994. Pickup trucks made up 18 percent of the vehicle stock in 1994, the same as in 1998. Aside from passenger cars, only large vans decreased in both number and share of total stock, dropping from 3 percent of the total vehicle stock to 2 percent over the 6-year period.

\section{What is the Significance of the Findings?}

The survey results have implications for the overall fuel economy in the United States and the amount of motor gasoline consumed. The increase in the number of minivans, sport-utility vehicles, and pickup trucks may depress overall fleet fuel economy, because these vehicles are subject to the fuel economy standards for light trucks and consume more fuel per mile traveled. Fuel economy is increased, however, by the retirement of older vehicles that are less fuel efficient than newer models. Passenger cars built after 1979 showed a dramatic increase in fuel economy, which rose 3 miles per gallon between 1979 and 1980 and then increased steadily—though less dramatically—throughout the $1980{ }^{\prime}$ s. $^{1}$ Fuel economy has leveled off in the 1990's. In 1991, 35 million vehicles, or 23 percent of the total vehicle stock, were from model year 1979 or earlier. By 1994, that number had dropped to 20 million, or 13 percent of the vehicle stock. Those older vehicles tend to be driven fewer miles than the new vehicles that replaced them, according to the survey data. Therefore, although older vehicles tend to consume more fuel per mile, their effect on the fleet average is mitigated by the fact that they are driven fewer miles. 
Household Vehicles Energy Consumption 1994 examines the effects of household size, household income, age of primary driver, vehicle characteristics, and Census region on household vehicle stock, miles traveled, fuel economy, and fuel consumption (Table 1.1). In general, the size of the household, composition of the household, and household income had a large effect on those variables.

\section{What is the Purpose of the Survey Report?}

The purpose of this report is to provide information on the use of energy in residential vehicles in the 50 States and the District of Columbia. Included are data about the number and types of vehicles in the residential sector, the characteristics of those vehicles, the total annual vehicle-miles traveled, per-household and per-vehicle vehicle-miles traveled, vehicle fuel consumption and expenditures, and vehicle-fuel economy.

The Energy Information Administration (EIA) is mandated by Congress to collect, analyze, and disseminate impartial, comprehensive data about energy: how much is produced, who uses it, and the purposes for which it is used. To comply with this mandate, EIA collects energy data from a variety of sources covering a wide range of topics. ${ }^{2}$

\section{How Was the Survey Conducted?}

The data for this report are based on personal interviews and telephone interviews with householders from the 1993 Residential Energy Consumption Survey (RECS) and the 1994 RTECS, conducted from 1993 through early 1995. The 1994 RTECS represents 96.6 million households, of which 84.9 million owned or had access to 156.8 million household motor vehicles in the 50 States and the District of Columbia.

The beginning-of-year data collection for the RTECS was combined with the 1993 RECS personal interviews in the fall of 1993. In 1994 and 1995, further data about the vehicle stock and vehicle-miles traveled were collected by telephone interviews. Mail questionnaires were sent to households that could not be contacted by telephone. Mid-year data collection during 1994 was conducted to identify vehicles acquired or disposed of during the first half of the year and to obtain estimated beginning or final odometer readings on those vehicles. The end-of-year data collection was conducted in the first 4 months of 1995. At that time final odometer readings and changes in vehicle stock were collected.

The survey was used to collect data on actual vehicle-miles traveled for each vehicle in a household by obtaining the odometer reading at two points in time. Vehicle characteristic information (type of vehicle, engine size, number of cylinders, type of fuel system, etc.) was collected directly from respondents and from decoded Vehicle Identification Numbers. Vehicle fuel consumption and expenditures were estimated using vehicle fuel economies as calculated (and adjusted) by the Environmental Protection Agency and presented in miles per gallon and by motor fuel prices collected by the Bureau of Labor Statistics.

\section{Are the Survey Results Statistically Significant?}

Because the statistics published in this report are based on a sample of all residential housing units in the 50 States and the District of Columbia as of November 1993, the values are estimates rather than exact measures for the population. Certain estimates in these tables are suppressed due to large error levels or few sample observations. (See table footnotes for explanation.) Each table in the "Detailed Tables" section includes row and column relative standard error (RSE) factors to be used in calculating RSEs for individual table entries.

Unless stated otherwise, all comparisons reported in the text are statistically significant, based on a standard test made at the 0.05 significance level. These tests were made using the actual RSE's computed by EIA. No adjustments were made for simultaneous inference. 


\begin{tabular}{|c|c|c|c|c|}
\hline \multicolumn{5}{|c|}{$\begin{array}{l}\text { Table 1.1. Household Characteristics, Vehicle-Miles Traveled, Fuel } \\
\text { Consumption, and Fuel Expenditures per Household, } 1994\end{array}$} \\
\hline $\begin{array}{l}\text { Household } \\
\text { Characteristics }\end{array}$ & $\begin{array}{l}\text { Average } \\
\text { Number of } \\
\text { Vehicles per } \\
\text { Household }\end{array}$ & $\begin{array}{l}\text { Average } \\
\text { Vehicle-Miles } \\
\text { Traveled per } \\
\text { Household } \\
\text { (thousands) }\end{array}$ & $\begin{array}{l}\text { Molor Fuel } \\
\text { Consumption } \\
\text { per Household } \\
\text { (gallons) }\end{array}$ & $\begin{array}{l}\text { Motor Fuel } \\
\text { Expenditures } \\
\text { per Household } \\
\text { (dollars) }\end{array}$ \\
\hline \multicolumn{5}{|l|}{ Household Size } \\
\hline \multicolumn{5}{|l|}{1 person } \\
\hline \multicolumn{5}{|l|}{2 persons } \\
\hline \multicolumn{5}{|l|}{3 persons } \\
\hline \multicolumn{5}{|l|}{4 persons } \\
\hline \multicolumn{5}{|l|}{5 persons } \\
\hline \multicolumn{5}{|l|}{6 or more persons } \\
\hline \multicolumn{5}{|l|}{$\begin{array}{l}\text { Household } \\
\text { Composition }\end{array}$} \\
\hline \multicolumn{5}{|l|}{$\begin{array}{l}\text { Households with } \\
\text { Children }\end{array}$} \\
\hline \multicolumn{5}{|l|}{$\begin{array}{l}\text { Households with } \\
\text { No Children }\end{array}$} \\
\hline \multicolumn{5}{|l|}{ Family Income } \\
\hline \multicolumn{5}{|l|}{ Less than $\$ 5,000$} \\
\hline \multicolumn{5}{|l|}{$\$ 5,000-\$ 9,999$} \\
\hline \multicolumn{5}{|l|}{$\$ 10,000-14,999$} \\
\hline \multicolumn{5}{|l|}{$\$ 15,000-19,000$} \\
\hline \multicolumn{5}{|l|}{$\$ 20,000-24,999$} \\
\hline \multicolumn{5}{|l|}{$\$ 25,000-34,999$} \\
\hline \multicolumn{5}{|l|}{$\$ 35,000-49,999$} \\
\hline \multicolumn{5}{|l|}{$\$ 50,000-74,999$} \\
\hline$\$ 75,000$ or more & 2.3 & 28.5 & 1,443 & 1,692 \\
\hline
\end{tabular}

Source: Table 5.2 in this report. 


\section{How Is This Report Organized?}

A discussion of the highlights of survey findings, featuring tables and figures that present information of special interest or that provide a finer analysis than is contained in the detailed tables, follows this section. The "Detailed Tables" section that follows the main text contains extensive cross-tabulations of household characteristics, vehicle characteristics, and vehicle fuel consumption and expenditures. Definitions of the terms used in this report are located in the Glossary.

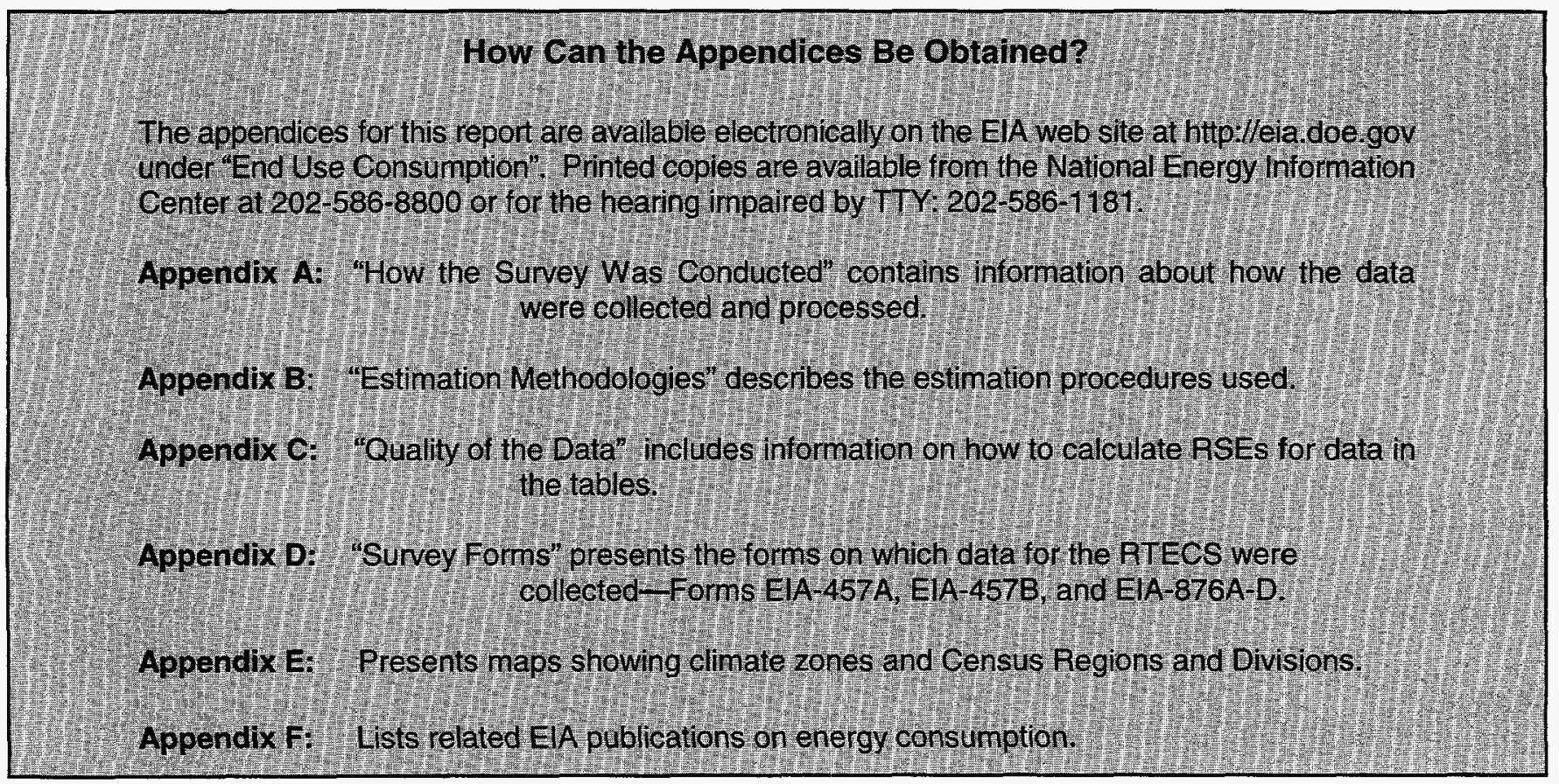

\section{Thank You ...}

EIA gratefully acknowledges the cooperation of the respondents in supplying the information used to produce the estimates presented here. 


\section{Chapter 2. Vehicle Characteristics}

U.S. households used a fleet of nearly 157 million vehicles in 1994. Despite remarkable growth in the number of minivans and sport-utility vehicles, passenger cars continued to predominate in the residential vehicle fleet. This chapter looks at changes in the composition of the residential fleet in 1994 compared with earlier years and reviews the effect of technological changes on fuel efficiency (how efficiently a vehicle engine processes motor fuel) and fuel economy (how far a vehicle travels on a given amount of fuel). Using data unique to the Residential Transportation Energy Consumption Survey, it also explores the relationship between residential vehicle use and family income.

\section{Changes in the Composition of the Residential Fleet}

The number of vehicles in U.S. households rose from 148 million in 1988 to 157 million in 1994, an increase of 9 million vehicles. During the same period, the U.S. population grew by almost 15 million persons to 260 million. ${ }^{3}$ The average annual growth rates of both residential vehicles and population equaled 1.0 percent. The number of licensed drivers grew ${ }^{4}$ at an average annual rate of 1.2 percent, and, in 1994, there were 1.1 licensed drivers per residential vehicle, slightly more than in 1988.

Residential vehicles continued to account for the majority of all U.S. vehicles. Not surprisingly, the rate of increase in vehicles available for use in households was approximately the same as the rate of increase for the U.S. total vehicle stock (Figure 2.1).

\section{A Growing Number of Passenger Cars Were Replaced by Light Trucks}

The number of passenger cars in U.S. households was 106 million in 1994 (Figure 2.2). At a 68-percent share, passenger cars continued to dominate the residential fleet, but not to the same extent as in 1988, when their share was 74 percent.

In contrast, there was remarkable growth in the number of household vehicles categorized as light trucks, particularly minivans. From 1988 through 1994, the number of light trucks in the residential fleet increased by an estimated 12 million, which more than compensated for the apparent decline in the number of passenger cars.

The number of minivans grew dramatically. It rose from 2.2 million in 1988 to 8.1 million in 1994, an increase of 268 percent (Figure 2.3). Minivans, introduced into the market in the mid-1980's, accounted for nearly 1 in 20 residential vehicles in 1994.

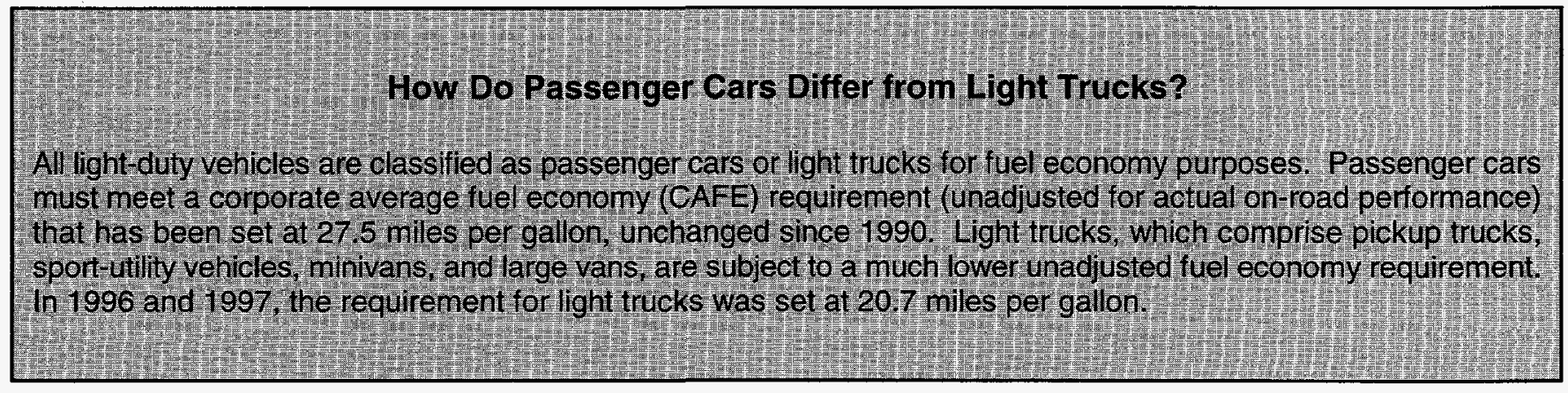


Figure 2.1 Number of Vehicles, 1983, 1985, 1988, 1991, and 1994

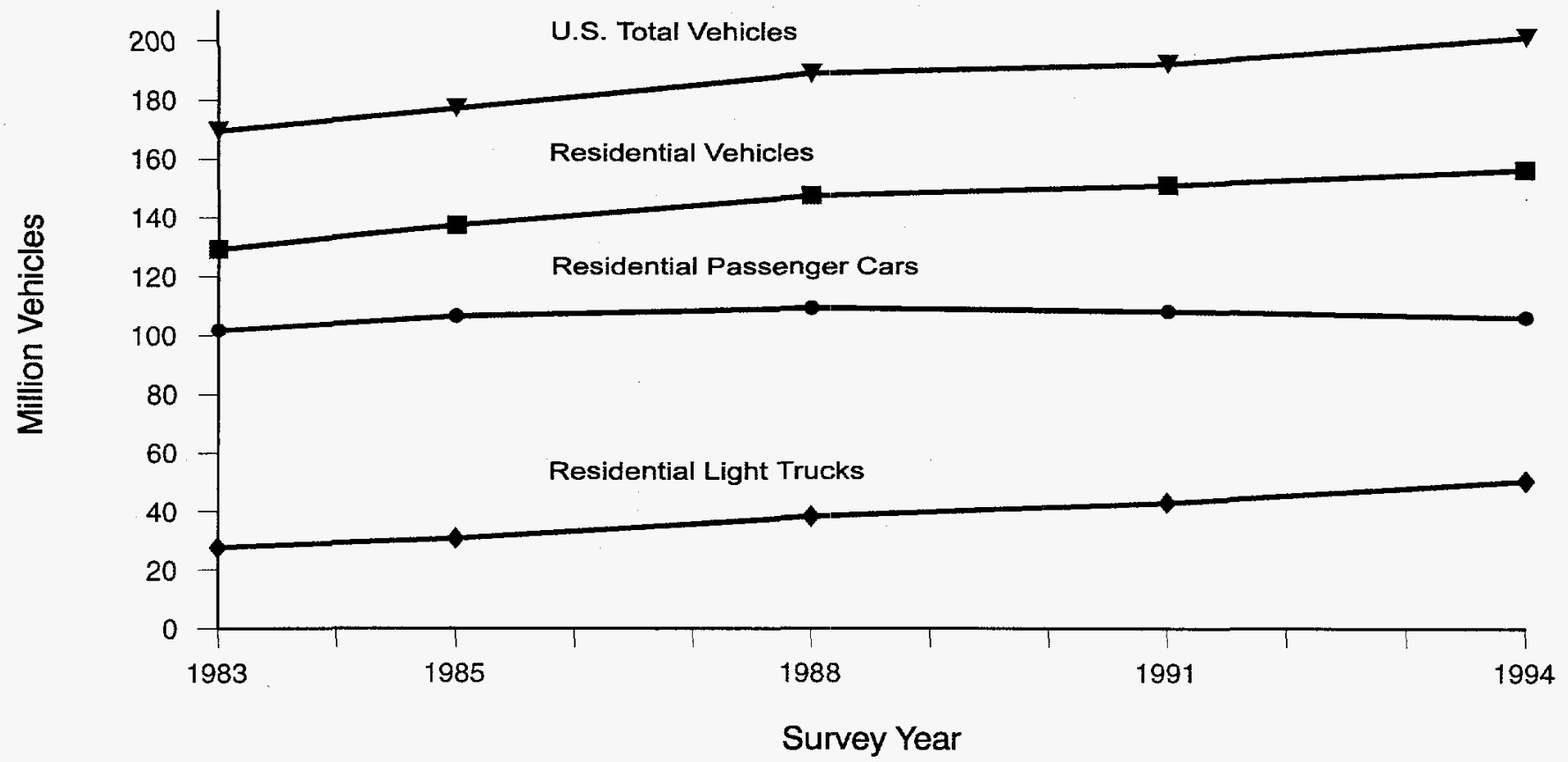

Note: U.S. total vehicles include motorcycles and buses, as well as other nonresidential vehicles, which are excluded from the Residential Transportation Energy Consumption Survey.

Sources: U.S. Total Vehicles: Energy Information Administration (EIA), Annual Energy Review 1995, DOE/EIA-0384(95) (Washington, DC, July 1996), Table 2.16. Residential Vehicles: - 1983-EIA, Consumption Patterns of Household Vehicles 1983, DOEJEIA-0464(83) (Washington, DC, January 1985), Table 11. • 1985-EIA, Consumption Patterns of Household Vehicles 1985, DOE/EIA-0464(85) (Washington, DC, April 1987), Table 8. - 1988-EIA, Household Vehicles Energy Consumption 1988, DOE/EIA-0464(88) (Washington, DC, February 1990), Table 6. • 1991-EIA, Household Vehicles Energy Consumption 1991, DOE/EIA-0464(91) (Washington, DC, December 1993), Table 8. $\bullet 1994-T a b l e ~ 5.1$ in this report.

Note: Totals may not equal sum of components due to independent rounding.

Figure 2.2 Number of Residential Vehicles by Type, 1988 and 1994

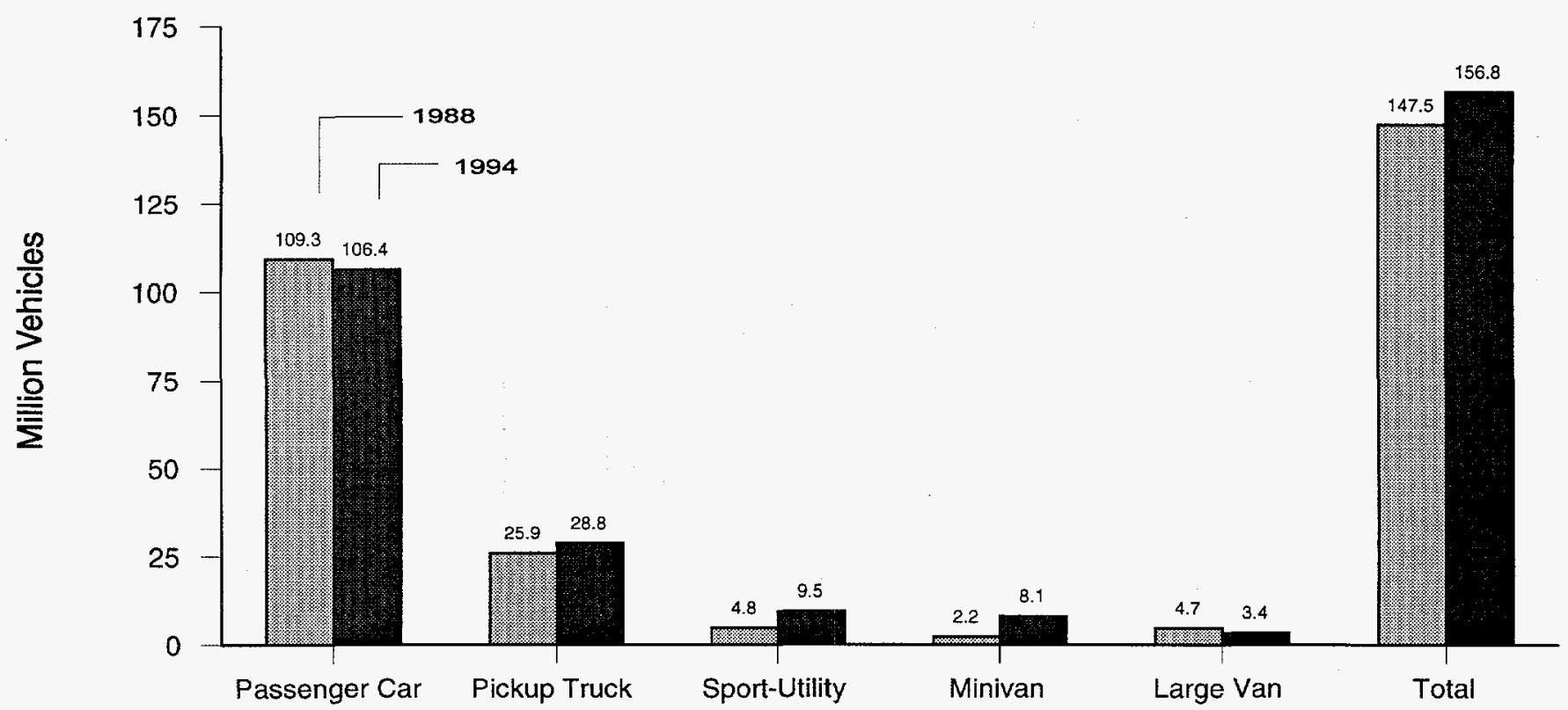

Vehicle Type

Sources: - 1988-Energy Information Administration, Household Vehicles Energy Consumption 1988, DOE/EIA-0464(88) (Washington, DC, February 1990), Table 6. 1994-Table 5.1 in this report. 


\section{Figure 2.3 Change in Number of Residential Vehicles by Type, 1988 to 1994}

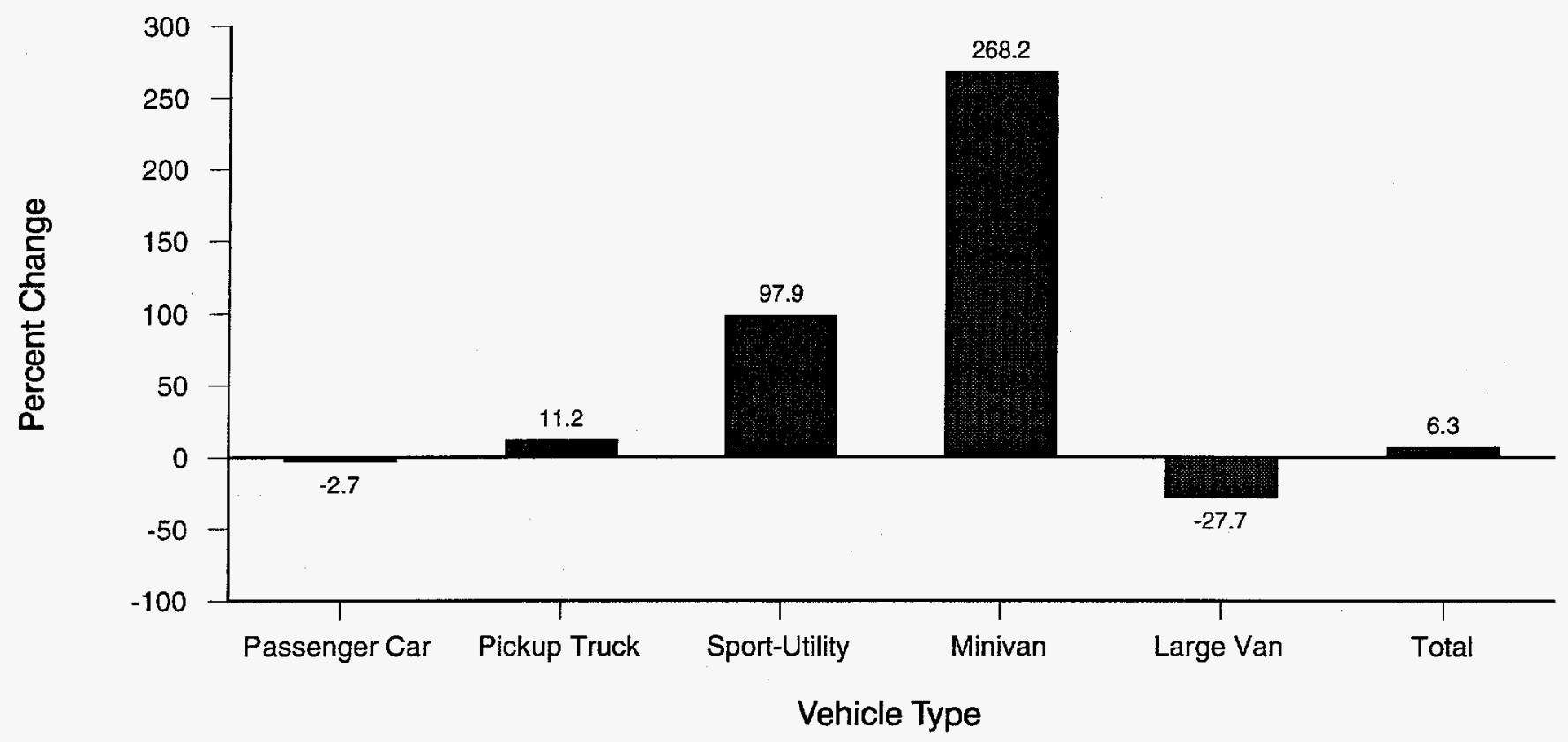

Sources: • 1988-Energy Information Administration, Household Vehicles Energy Consumption 1988, DOE/EIA-0464(88) (Washington, DC, February 1990), Table 6. • 1994-Table 5.1 in this report.

Sport-utility vehicles, termed "jeep-like" vehicles in the 1988 survey, also increased in number at a remarkable rate. From 1988 through 1994, they rose 98 percent to 9.5 million.

Pickup trucks were the most prevalent of the light trucks on the road. Almost 1 in 5 residential vehicles in 1994 was a pickup truck. In 1994, the number of pickup trucks totaled 29 million.

The only type of light truck estimated to have declined over the period was the large van. The large van share of the residential fleet fell from 3 percent in 1988 to 2 percent in 1994 when large vans numbered 3.4 million.

Throughout the 7-year period of 1988 through 1994, inflation-adjusted prices of motor fuel (motor gasoline and diesel fuel) were well below the peak prices of the early 1980's. For example, at its peak in 1981, the adjusted price (in chained [1992] dollars ${ }^{5}$ and including taxes) of unleaded regular motor gasoline was $\$ 2.09$ per gallon, whereas in 1994 the comparable price was $\$ 1.06$. $^{6}$

When motor fuel prices are relatively low, consumers have less incentive for choosing vehicles with higher fuel economy ratings. And, in fact, consumer preference for light trucks, which have significantly lower fuel economy ratings than do passenger cars, was one factor leading to the decline in the number of passenger cars. A second factor contributing to the increase in light trucks' share of the residential fleet was the continuing tendency of householders to keep older light trucks in operation while retiring older passenger cars. Those factors had their greatest effect in the West, where light trucks make up a higher percentage of the residential vehicle fleet than in other regions.

\section{Consumer Preference for Light Trucks Increased}

Total sales of new light trucks to all sectors rose during the 1980's and 1990's (Figure 2.4). For model year 1995, 5.7 million light trucks were sold, more than ever before and nearly three times the number of sales for model year 1980 . Although sales of new passenger cars fluctuated and rose as high as 11 million for model year 1986, for model year 


\section{Figure 2.4 Sales of New Passenger Cars and New Light Trucks for Model Years $1980-1995$}

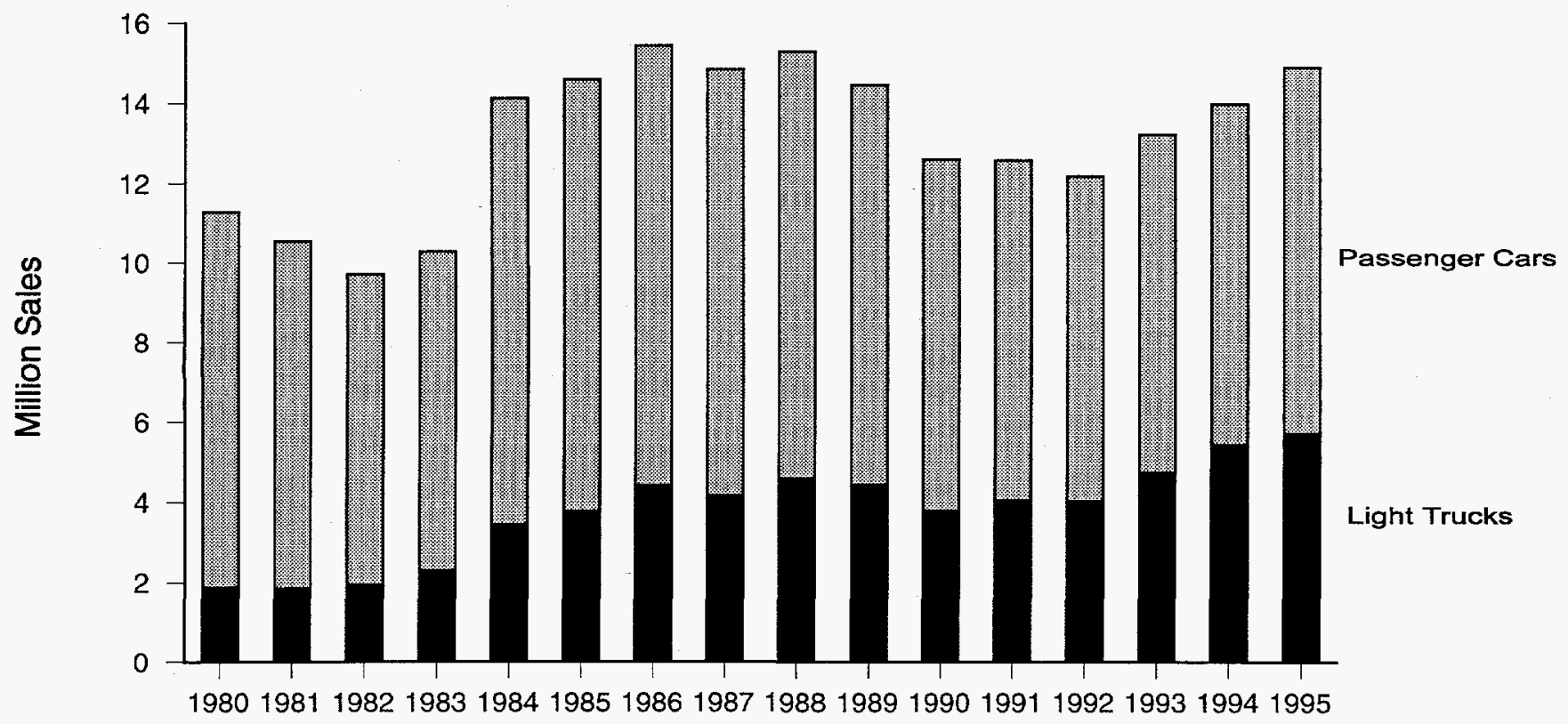

Source: Federal Highway Administration, Summary of Fuel Economy Performance (Washington, DC, April 1996), p. 3.

1995 sales of 9.2 million were about the same as they had been for model year 1980 (9.4 million). For model year 1995 , therefore, light trucks accounted for slightly more than one-third of total sales.

Sales data disaggregated by sector are not available, but changes in the composition of the residential fleet, as noted above, clearly reflect householders' growing preference for light trucks. That preference restrained the increase in the fuel economy of the residential fleet as a whole (see Chapter 4).

\section{Light Trucks Were Kept in Operation Longer}

Although the number of old passenger cars in the residential fleet declined rapidly from 1988 through 1994, old light trucks, particularly pickup trucks and large vans, were taken out of service at a slower rate. For example, the number of passenger cars of model year 1982 or earlier fell from 57 million in 1988 to 20 million in 1994, meaning that about two-thirds of those older passenger cars were retired from the residential fleet over the 7-year period (Figure 2.5). By comparison, the number of pre-1983 light trucks fell from 21 million to 11 million, meaning that only about one-half were retired.

The tendency to retain old light trucks led to a difference in the average age of light trucks and passenger cars. In the 1994 fleet, the average age of passengers cars was 8.1 years. Light trucks as a group averaged 8.5 years, but there was wide variation in the average age of different types of light trucks. For example, pickup trucks and large vans were, on average, 9.9 years old, whereas sport-utility vehicles averaged 6.6 years and minivans averaged 4.8 years.

\section{Light Trucks Were Most Prevalent in the West}

In 1994, the western fleet had a high ratio of light trucks to passenger cars. Light trucks made up 37 percent of the residential fleet in the West, compared with 33 percent in the South, 30 percent in the Midwest, and 26 percent in the Northeast (Figure 2.6). 
Figure 2.5 Number of Pre-1983 Residential Vehicles by Type, 1988, 1991, and 1994

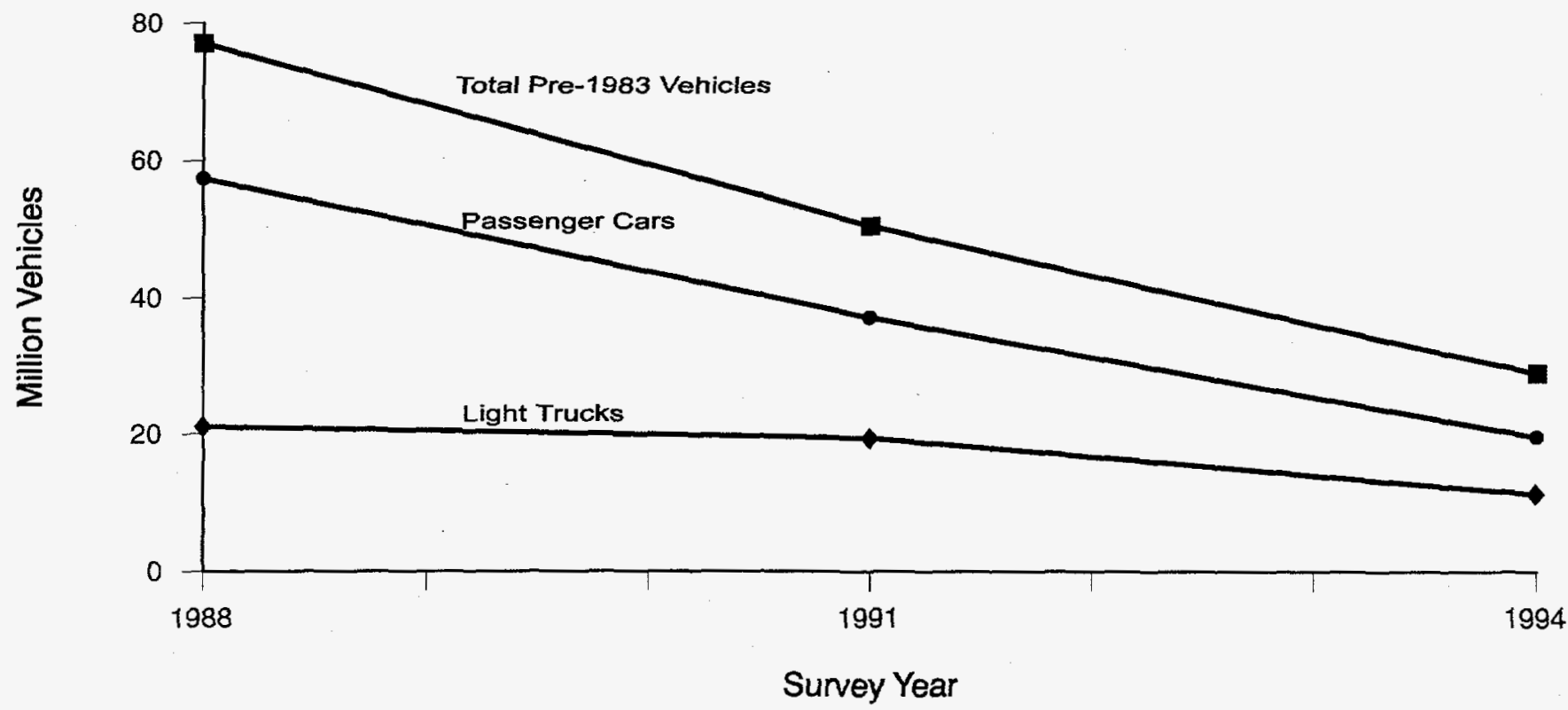

Sources: - 1988-Energy Information Administration (EIA), Household Vehicles Energy Consumption 1988, DOE/EIA-0464(88) (Washington, DC, February 1990), Table 9. - 1991-EIA, Household Vehicles Energy Consumption 1991, DOE/EIA-0464(91) (Washington, DC, December 1993), Table 11. $1994-$ Table 5.4 in this report.

Figure 2.6 Light Trucks' Share of Each Region's Residential Fleet, 1994 (Percent)
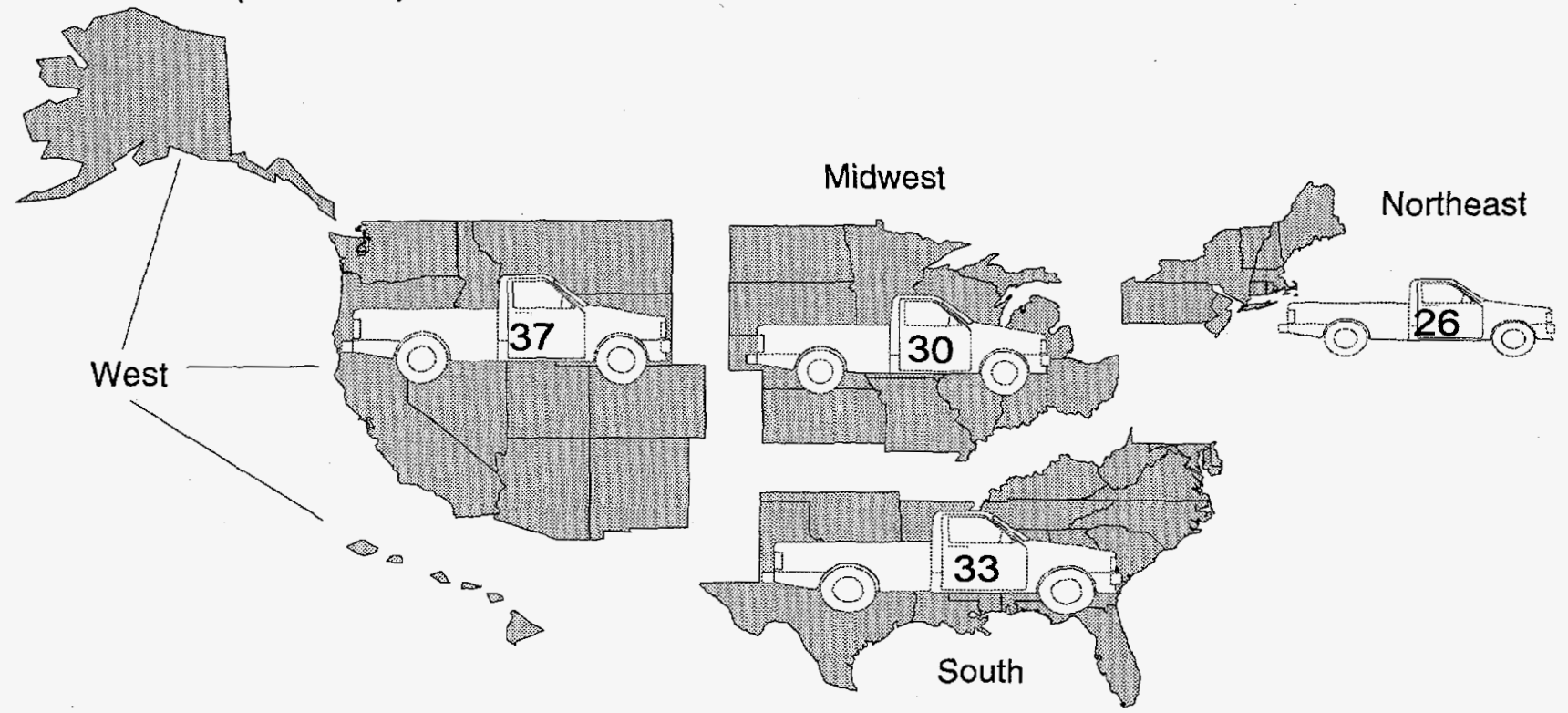

Source: Table 5.16 in this report. 
The national tendency to keep old trucks in service longer than old cars meant that in the West old residential vehicles as a whole were taken out of service at a slower rate than in the other regions of the country. For example, vehicles of model year 1988 or earlier accounted for a 64-percent share of all of the residential vehicles in the West. In the other regions of the country, the shares were lower: 59 percent in the Midwest and 57 percent each in the South and Northeast.

The rural nature of much of the West may have contributed to the prevalence of light trucks in the region. In general, rural areas had a higher ratio of light trucks to passenger cars than did urban areas. Even California, the most populous State in the West, had a ratio above the national average. In California, 1 of every 3 residential vehicles was a light truck. In contrast, in New York fewer than 1 in 4 was.

\section{Residential Vehicles at the Household Level}

In 1994, the 156.8 million residential vehicles were divided among the 84.9 million households that had at least one vehicle, meaning that each of those households had an average of about 1.8 vehicles. Despite the increase in the total number of vehicles in the residential fleet from 1988 to 1994, the average number of vehicles per household remained at 1.8 because the growth in the number of households kept pace with the growth in the number of vehicles. An additional $\mathbf{1 2 . 5}$ million households, including many of the poorest households, had no vehicles.

\section{Lower-Income Households Had Fewer Vehicles in 1994}

Lower-income households - those with annual family incomes below $\$ 25,000$-had, on average, 1.5 vehicles in 1994 . Not surprisingly, households with annual family incomes of $\$ 25,000$ or above had more vehicles -2.1 , on average.

\section{More Households in 1994 Had No Vehicles}

In 1988 and 1991, the number of households without vehicles remained at about 10 million, even though the total number of households rose 3.0 million from 1988 to 1991 . In 1991, the share of households without vehicles was 10.6 percent.

In 1994 , by contrast, the share of households without vehicles jumped to 12.7 percent. The total number of households rose 2.7 million from 1991 to 1994, while the number of households without vehicles rose 2.5 million.

The lowest-income households accounted for 83 percent of the total increase in the number of households without vehicles. (The lowest-income households are those categorized as eligible for Federal assistance because their income is lower than 150 percent of the national poverty line or 60 percent of statewide median income, whichever is higher. ${ }^{7}$ ) In 1994, 30 percent of the Nation's lowest-income households were without vehicles, compared with a national average for all households of 13 percent.

\section{Changes in Residential Vehicle Technology}

The transportation sector relies almost entirely on petroleum. Because it also accounts for about two-thirds of U.S. total petroleum demand, ${ }^{8}$ passenger cars and light trucks became a focus of efforts to use petroleum more efficiently, thereby restraining demand.

The Energy Policy and Conservation Act of 1975 required passenger car and light truck manufacturers to meet corporate average fuel economy (CAFE) standards applied on a fleet-wide basis for each manufacturer. ${ }^{9}$ The CAFE standards, higher fuel prices in the 1970's and 1980's, and environmental quality initiatives such as the Clean Air Act Amendments of 1990 all contributed to increasing the demand for more fuel-efficient vehicles. 
In turn, the demand for more fuel-efficient vehicles spurred improvements in existing technologies and the development of new technologies. For example, using lighter-weight materials and reducing the size of vehicles led to lighter vehicles that consumed less fuel per mile.

Many improvements occurred in engine technology. Increasing the number of valves per cylinder resulted in increased performance from smaller, more fuel-efficient engines. Increasing the number of gears in manual and automatic transmissions allowed engines to operate at peak efficiency more of the time. Similarly, using lockup torque converters to allow direct drive under some driving conditions also increased fuel economy.

One of the most far-reaching improvements in engine technology concerned fuel injection, which was available but not widely used prior to the mid-1980's. Valued for its greater fuel economy, as well as for its ability to control carbon monoxide emissions and to improve engine performance, fuel injection technology began to penetrate the fleet during the 1980's, and it was improved to such an extent that after 1990 virtually all new light-duty vehicles were equipped with fuel injection instead of carburetors. By 1994, the trend toward the use of fuel injection rather than carburetors for fuel metering had brought the share of residential vehicles using fuel injection to nearly half of the total residential fleet. That share will increase rapidly in future years as older vehicles are retired.

\section{Engine Size Shrank and Then Increased Slowly}

For model year 1975 (the year in which CAFE standards were first mandated), residential vehicle engine size averaged 293 cubic inches of displacement. ${ }^{10}$ Twelve years later, the engines were much smaller. Model year 1987 engines averaged 175 cubic inches (Figure 2.7). Thereafter, engine size trended upward, reaching an average of 198 cubic inches of displacement for model year 1995.

As is generally the case, changes in the residential vehicle fleet as a whole lagged the changes by model year. Of the 3 years for which fleet data are available, 1991 had the lowest average engine size (despite an upward trend in the 4 preceding model years). In 1994, the fleet average was 227 cubic inches, while the 1994 model year average was 196.

\section{Engines Averaged Fewer Cylinders}

Meanwhile, the average number of cylinders in residential vehicle engines was decreasing. In 1988, 55 million vehicles, over one-third of the total residential fleet, had 8-cylinder engines. In 1994, 8-cylinder engines were found in only about 42 million vehicles, just over one-fourth of the total (Figure 2.8).

The trend over the 7-year period was toward more 4- and 6-cylinder engines. In 1994, 4-cylinder engines were the most common (62 million out of a total of 157 million), but the 52 million 6-cylinder engines represented the greatest increase ( 45 percent) relative to the 1988 level.

\section{Power and Performance Improved but Fuel Economy Stagnated}

The improvements in residential vehicle technology allowed for improvements in performance despite shrinking or relatively stable engine size. However, improved performance came at the expense of improvements in fuel economy. For example, technical improvement in the efficiency of engines can be used to provide increased acceleration or smaller, lighter engines with better fuel economy.

The effect of the improvements was particularly noticeable over the 1988-to-1994 period, when engine size stayed at or below 198 cubic inches of displacement but power and performance rose markedly. For example, the average horsepower of new vehicles rose from 123 in 1988 to 159 in 1994, an increase of 29 percent (Figure 2.9). Vehicles manufactured in 1994, with an average of 159 horsepower, had the highest horsepower rating in at least 20 years, a rating markedly higher than the 20-year low-point of 102 horsepower in 1981. 
Figure 2.7 Average Engine Size for Residential Vehicles for Model $1980-1995$

Years

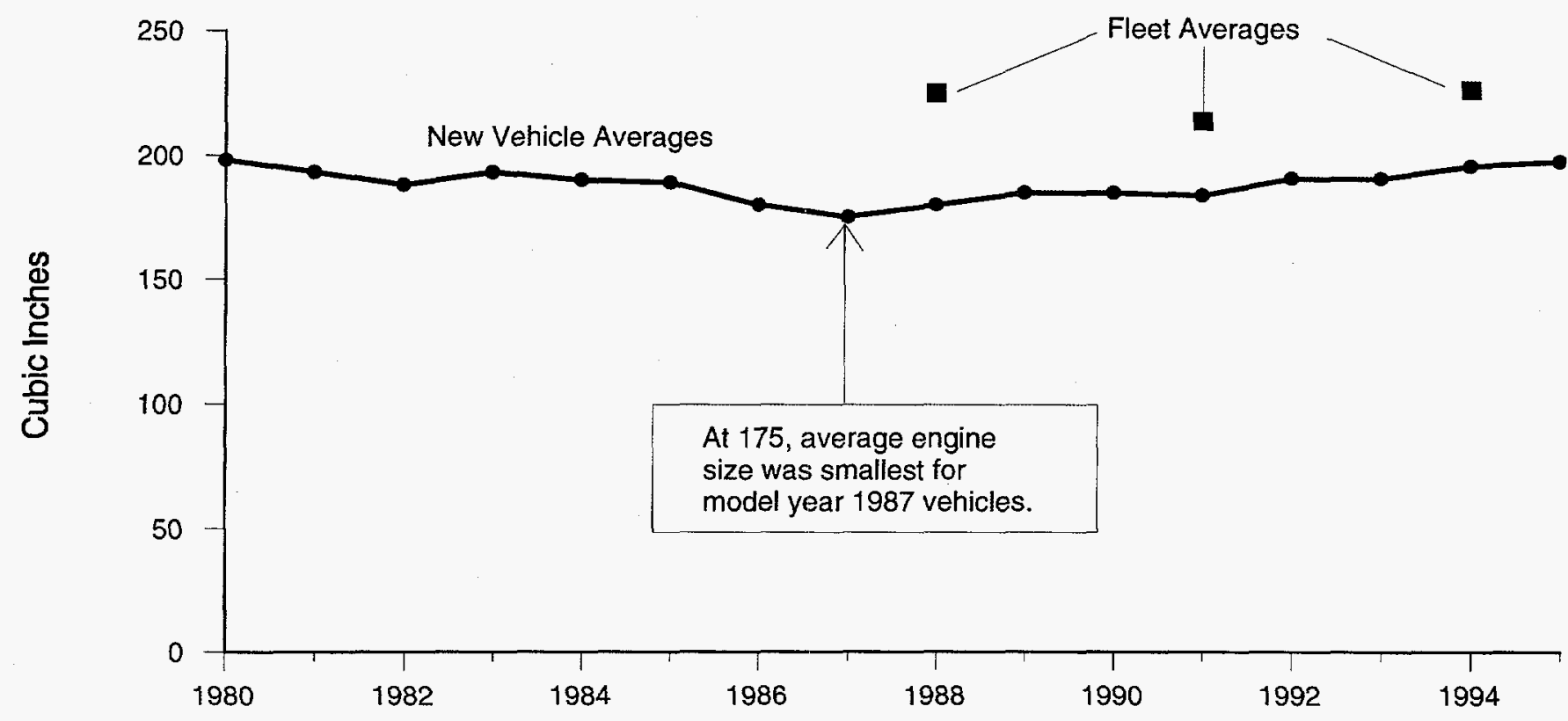

Sources: New Vehicle Averages: Environmental Protection Agency, Light-Duty Automotive Technology and Fuel Economy Trends Through 1996, EPA/AATDSG/96-01 (Washington, DC, August 1996), Table 1. Fleet Averages: Energy Information Administration calculations made on the basis of data from the Residential Transportation Energy Consumption Survey 1988, 1991, and 1994, March 17, 1997.

Figure 2.8 Number of Cylinders in Residential Vehicle Engines, 1988 and 1994

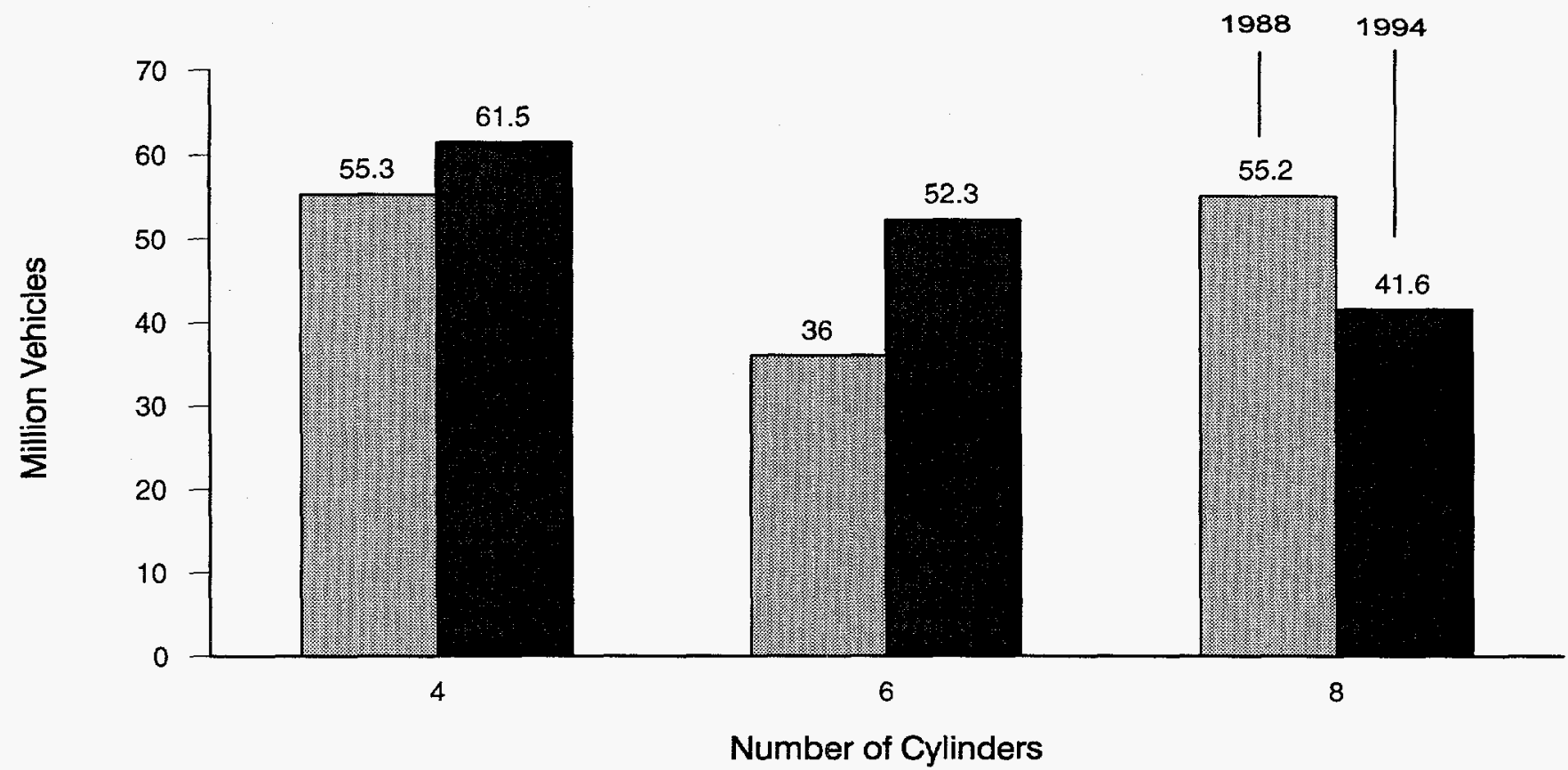

Sources: - 1988-Energy Information Administration, Household Vehicles Energy Consumption 1988, DOE/EIA-0464(88) (Washington, DC, February 1990), Table 6.

- 1994-Table 5.1 in this report. 
Similarly, the time required for the average residential vehicle to accelerate from 0 miles per hour to 60 miles per hour was reduced by 1.1 seconds, from 12.8 seconds in 1988 to 11.7 seconds in 1994 . The 11.7 seconds time was the fastest in at least 20 years. ${ }^{11}$

Over the same 7 years, however, the average fuel economy of new vehicles stagnated. Nevertheless, the average fuel economy of the residential fleet rose somewhat, as new, more fuel-economic vehicles replaced older, less fuel-economic vehicles (see Chapter 4).

Figure 2.9 Vehicle Performance for Model Years 1980 - 1995

$$
\text { (Index, } 1980=100)
$$

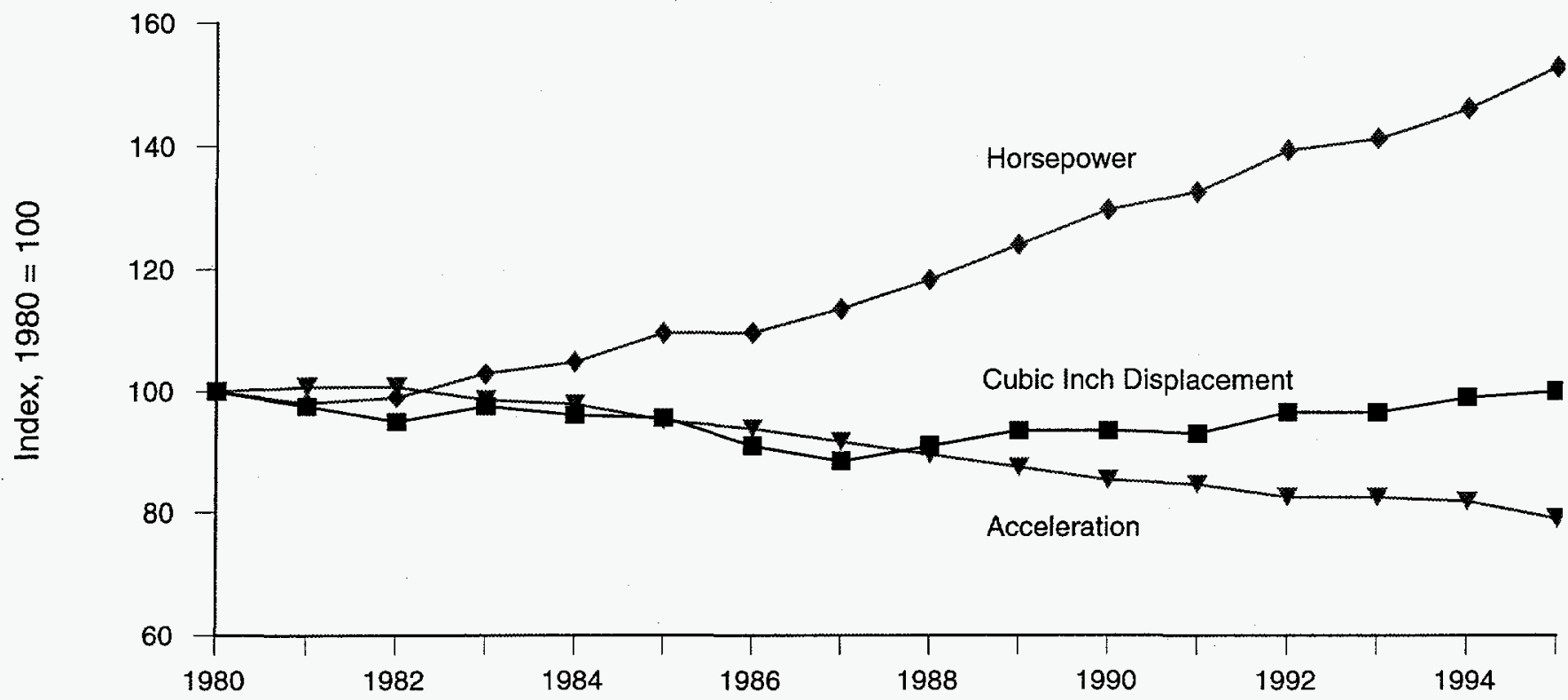

Source: Environmental Protection Agency, Light-Duty Automotive Technology and Fuel Economy Trends Through 1996, EPAJAATDSG/96-01 (Washington, DC, August 1996), Table 1. 



\section{Chapter 3. Vehicle-Miles Traveled}

Vehicle-miles traveled-the number of miles that residential vehicles are driven-is probably the most important information collected by the Residential Transportation Energy Consumption Survey. Using the data on vehicle-miles traveled allows analysts to answer such questions as:

- "Are minivans driven more than passenger cars?"

- "Do people in the West drive more than people elsewhere?"

- "Do people conserve their new cars by driving them less?"

- "Who drives more--people in households with children, or other people?"

- "At what ages do people drive the most?"

- "How does growing income affect the amount of driving?"

In addition to answering those kinds of questions, analysts also use the number of vehicle-miles traveled to compute estimated, on-road vehicle fuel consumption, economy, and expenditures, all of which have important implications for U.S. energy policy and national security (see Chapter 4).

\section{The Number of Vehicle-Miles Traveled Continued To Rise}

In 1994, U.S. residential vehicles traveled 1,793 billion miles (Figure 3.1), a distance equal to more than 70 million trips around the world. The amount of travel in 1994 was 282 billion miles more than in 1988. From 1988 through 1994, the average annual growth in the number of miles traveled was 2.9 percent, almost 3 times the rate of growth in the number of residential vehicles during that period.

An average vehicle, therefore, traveled farther in 1994 than in 1988: 11,400 miles per year compared with 10,200 miles per year (Figure 3.2). Because the number of vehicles per household remained steady at about 1.8 from 1988 through 1994, per-vehicle and per-household mileage grew at about same rate. The per-household average rose from 18,600 miles in 1988 to 21,100 miles in 1994.

But while the number of miles traveled and the number of residential vehicles increased, the total amount of paved and unpaved roadway remained at about 3.9 million miles. ${ }^{12}$ Most construction was aimed at improving, rather than extending, the existing roadway system, about 40 percent of which was unpaved as of 1994 . In 1988, the average mile of roadway was traveled by residential vehicles 1,066 times per day. In 1994, the number was 1,257, about 18 percent higher. (Because residential vehicles make up only about four-fifths of the U.S. total fleet, there was, in fact, substantially more total travel per mile of roadway.) These figures provide some indication of the increase in traffic congestion over the period. 
Figure 3.1 Residential Vehicle-Miles Traveled by Type of Vehicle, 1988, 1991, and 1994

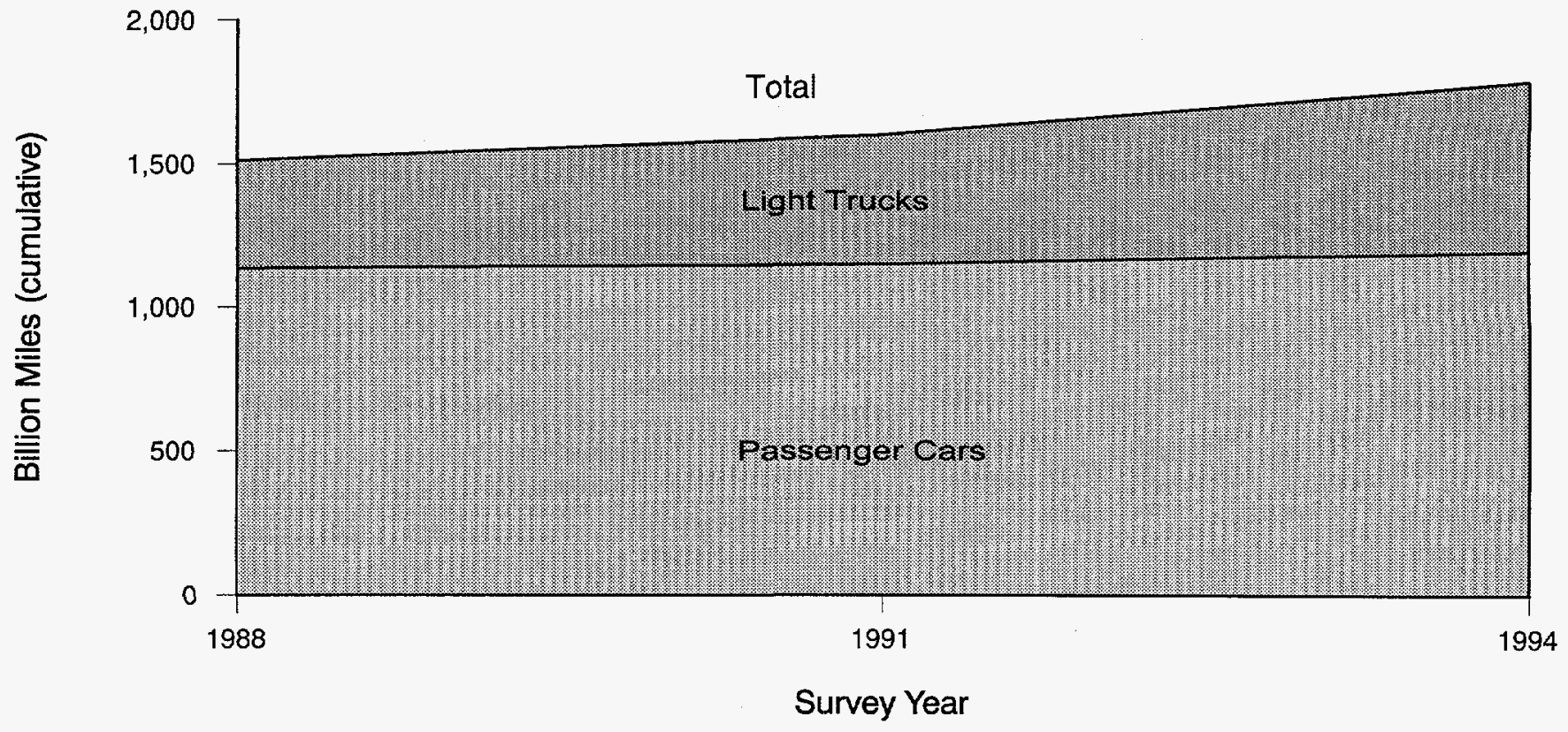

Sources: • 1988-Energy Information Administration (EIA), Household Vehicles Energy Consumption 1988, DOE/EIA-0464(88) (Washington, DC, February 1990), Table $12 \bullet 1991$-EIA, Household Vehicles Energy Consumption 1991, DOE/EIA-0464(91) (Washington, DC, December 1993), Table 14. $\bullet 1994-T a b l e ~ 5.7$ in this report.

Figure 3.2 Residential Vehicle-Miles Traveled per Household and per Vehicle by Survey Year, 1988, 1991, and 1994

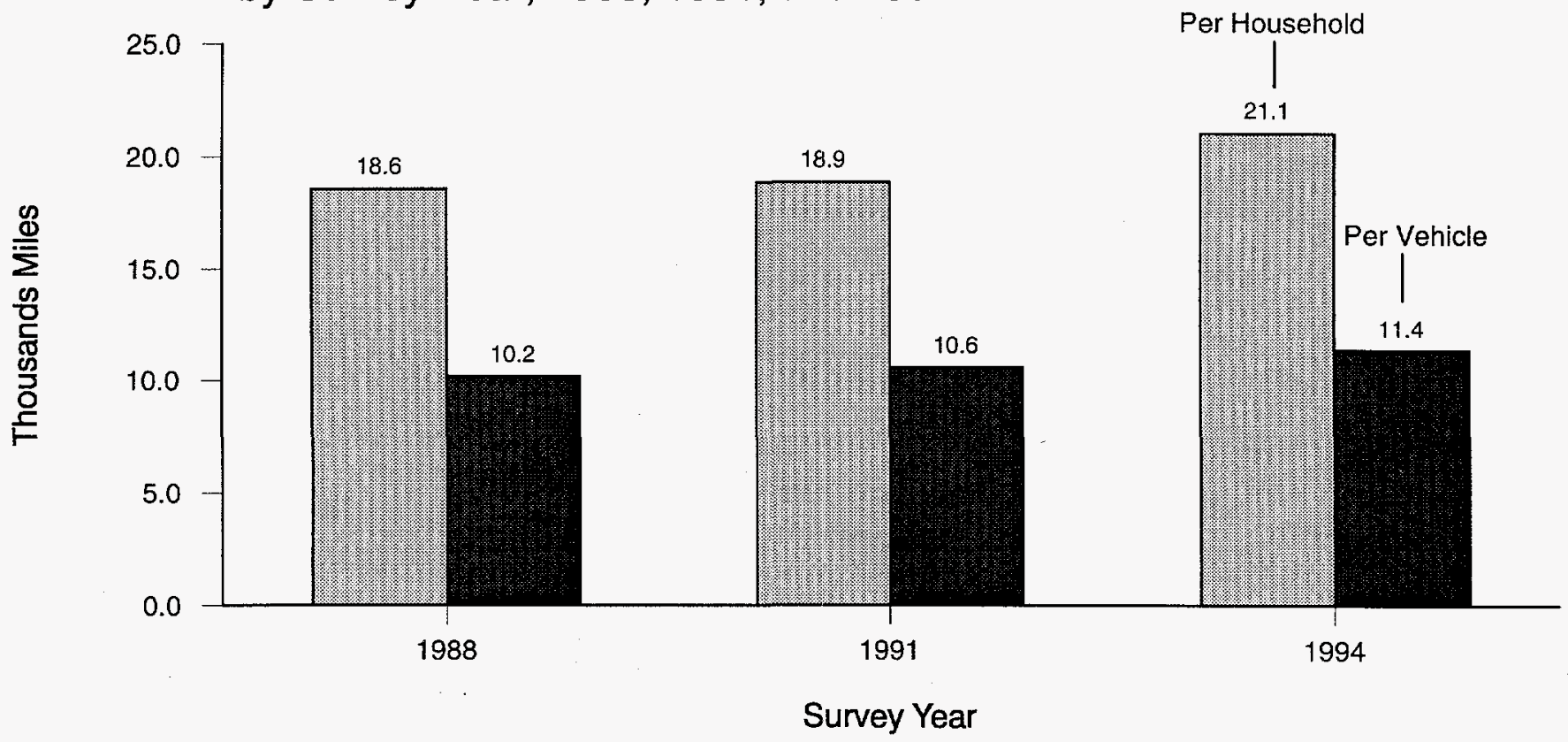

Sources: - 1988-Energy Information Administration, Household Vehicles Energy Consumption 1988, DOE/EIA-0464(88) (Washington, DC, February 1990), Tables 14 and 18. 1991 -EIA, Household Vehicles Energy Consumption 1991, DOE/EIA0464(91) (Washington, DC, December 1993), Tables 16 and 20. $1994-$ Tables 5.9 and 5.15 in this report. 


\section{How Are Vehicle-Miles Traveled Measured?}

The number of vehicle-miles traveled per year for each vehicle in the Residential Transportation Energy Consumption survey was obtained in one of two ways.

1. Calculations based on odometer readings. For each vehicle in the sample, the survey collected a beginningof-year and an end-of-year odometer reading. The number of vehicle-miles traveled annually is equal to the difference between the two readings, adjusted to reflect a 365-day year. The mileage for vehicles that were in the household less than a full year was adjusted to reflect the amount of time the vehicle was in the household.

2. Imputations using a regression estimate. For vehicles for which one or both odometer readings were missing, a regression estimate was used to estimate the annual mileage. As was done for the odometer reading calculations, the mileage for vehicles that were in the household less than a full year was adjusted to reflect the amount of time the vehicle was in the household.

The total number of vehicle-miles traveled, representing the number of miles traveled nationally by all residential vehicles, is equal to the weighted sum of the number of vehicle-miles traveled by each vehicle in the survey sample. (Each vehicle in the sample represents several thousand vehicles in the national residential fleet.)

\section{Miles Traveled by Light Trucks Increased the Fastest}

Largely because light trucks' share of the residential fleet increased from 1988 to 1994, the number of miles traveled by light trucks, rather than passenger cars, accounted for most of the increase in residential vehicle-miles traveled. Light trucks traveled 56 percent more miles in 1994 than they had in 1988, attaining an average growth rate of 7.8 percent per year. By comparison, the number of vehicle-miles traveled by passenger cars did not show a statistically significant change.

Light trucks' share of total vehicle-miles traveled rose from one-fourth in 1988 to one-third in 1994 . The increase was the result of two factors. Light trucks comprised a larger share of the residential fleet in 1994. In addition, two segments of the light truck fleet—minivans and sport utility-vehicles—were driven more miles per year per vehicle than were passenger cars.

\section{Minivans Were Driven the Most}

Minivans, which are owned primarily by families with children, tend to be driven more than other types of vehicles. In 1994, the average minivan was driven 13,400 miles, substantially more than the number of miles traveled, on average, by passenger cars, pickup trucks, and large vans. In general, households with children reported a higher number of vehicle-miles traveled than did other households (see section on "Inside the Average U.S. Household: Who Drives Most?").

Similarly, of the two types of passenger cars, station wagons were used more on average than were sedans. The average station wagon covered 12,100 miles in 1994 , compared with 11,200 miles traveled by the average sedan.

\section{Vehicles in the West Were Not Driven More Than in Other Regions}

It seems reasonable that people living in the "wide-open spaces" of the West would drive more than people living in urban areas of the West and in other regions of the country. However, the average number of miles traveled per vehicle in the West, 10,900 miles, was comparable to the average for the rest of the United States (Figure 3.3). That 
average was slightly lower than the averages in the South and the Midwest, but not statistically different from the average in the Northeast. The national average was 11,400 miles per vehicle in 1994.

However, the vehicle-miles-traveled averages may have masked significant variations in State and local areas, such as the less populous States located in the West. Other Energy Information Administration data sources, most notably, State-level motor gasoline consumption estimates, suggest wide variability in fuel use per capita. For example, motor gasoline consumption in the most fuel-intensive State (Wyoming) is more than twice that of consumption in the leastintensive State (New York). However, even in sparsely populated States such as Wyoming, most of the population lives in urban areas, so that a large part of personal travel could be local. Why then would motor gasoline use per capita vary so widely?

There are good reasons why per capita motor gasoline consumption may not be directly correlated with per capita vehicle-miles traveled. Estimates of total motor gasoline consumption could be greatly influenced by tourists visiting the State in numbers that are many times the size of the resident population. In addition, some western States with small populations have major cross-country interstate highways passing through. Vehicles traveling on such highways consume motor gasoline that is then counted in that State's consumption. Tourists and through traffic, largely unassociated with travel by State residents, would have the greatest relative effect on per capita ratios in States with large geographic areas and small populations. As an example, in 1994 over 7 million people visited Wyoming with its population of 476 thousand, according to the Wyoming Department of Tourism. In order for tourism to have had the same relative effect in California, almost everyone in the United States would have had to have visited California nearly two times in 1994.

\section{New Vehicles Are Driven More than Older Vehicles}

Vehicle age is closely correlated with the number of miles traveled: the newer the vehicle, the more miles it is driven, on average (Figure 3.4). In 1994, the newest vehicles (model years 1994 and 1995 $5^{13}$ ) were driven 14,300 miles, about 1.7 times as much as were the oldest vehicles (model years earlier than 1980, that is, vehicles at least 14 years old in 1994).

However, the difference between the number of miles traveled by the oldest and newest vehicles became much less pronounced in 1994 than it had been in 1988. In 1988, the newest vehicles were driven 12,900 miles, more than twice as much as were the oldest vehicles (those more than 14 years old in 1988). The likely explanation for this trend is that the oldest vehicles remaining in the residential fleet in 1994 were relatively newer, more fuel efficient, and probably more reliable than were the oldest vehicles in the 1988 fleet.

\section{Inside the Average U.S. Household: Who Drives the Most?}

People in the average U.S. household in 1994 drove their vehicles 21,100 miles, far enough to travel from New York City $^{14}$ to San Francisco seven times. That number, however, represents the average of about 85 million U.S. households with vehicles in 1994, and the average masks significant variation. For example, typical householder A, an older person whose children had left home, drove only 8,600 miles in 1994 . That same year, people in typical household B, which included teenagers of driving age, drove 29,900 miles. And people in household C, which also included teenagers of driving age and which had an income of $\$ 50,000$ or more, drove 40,200 miles.

By comparing data on vehicle-miles traveled per household with details about household size, composition, and income ${ }^{15}$ analysts can correlate the number of miles driven per household with factors such as how many people make up the household, how many children live in the household, and how much money the household earns. The Residential Transportation Energy Consumption Survey is the only source of that information. 
Figure 3.3 Residential Vehicle-Miles Traveled per Vehicle by Region, 1994 (Miles in Thousands)

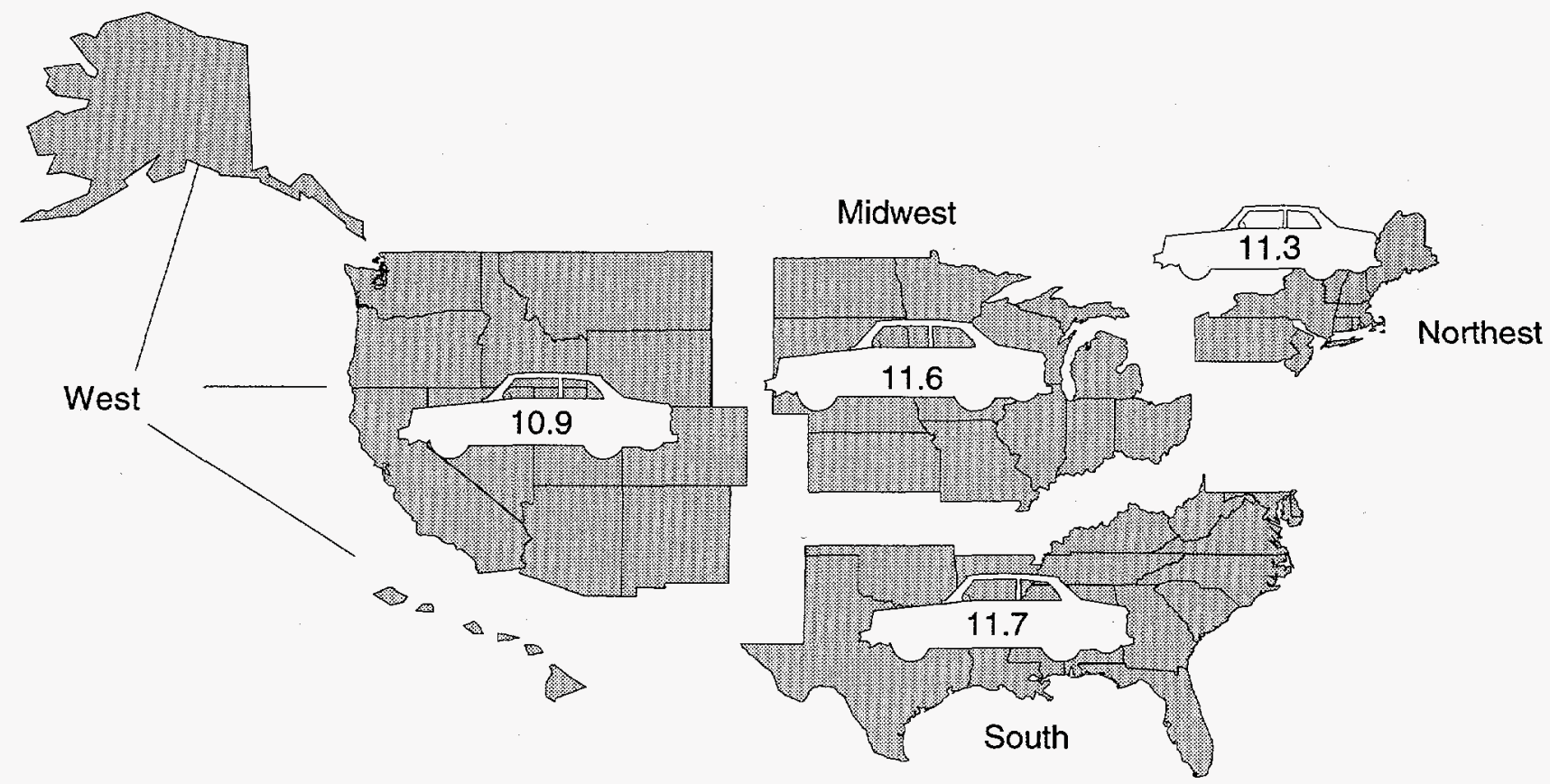

Source: Table 5.15 in this report.

Figure 3.4 Residential Vehicle-Miles Traveled per Vehicle by Vehicle Age, 1994

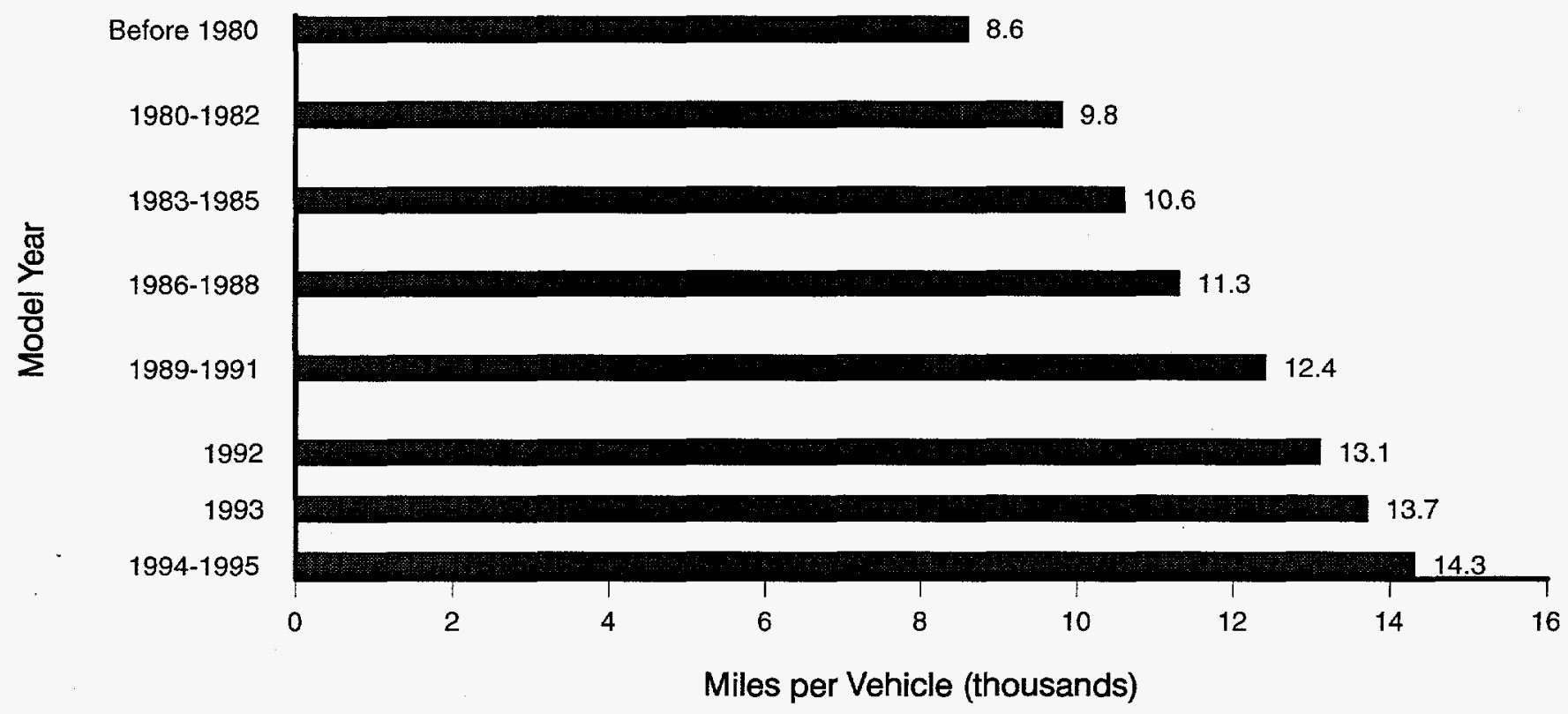

Source: Table 5.15 in this report. 


\section{Miles Driven Per Household Increased}

As explained in the previous section, in 1994, the number of vehicles per household, 1.8, was about the same as it had been in 1988, so that per-household mileage increased at the same rate as per-vehicle mileage. In 1988, the perhousehold average was 18,595 miles, compared with the 21,100 miles averaged in 1994.

People in households of all sizes drove more in 1994 than in 1988 (Figure 3.5). The difference between miles traveled in 1988 versus 1994 was dwarfed by the difference between miles traveled in the smallest households versus the largest. Travel in single-person households averaged 11,600 miles in 1994, about 800 miles more than in 1988. Travel in the typical household of four averaged 26,600 , about 3,300 miles more than in 1988 but 15,000 more than the smallest households.

As would be expected, the number of drivers in the household was an even more important influence on the number of miles traveled than was on the size of the household (Figure 3.6). Having three people in the household raised the number of vehicle-miles traveled by a factor of 2.2. Having three drivers in the household raised the number by a factor of 2.7. People in the average household with three drivers covered 33,100 vehicle-miles in 1994 .

\section{Teenagers in the Household Boosted Miles Traveled}

In general, people in households with children drive more, and those with driving-age children traveled the most vehicle-miles of any category in 1994: 29,900 miles (Figure 3.7). First, the presence of children of driving age tends to increase the number of drivers in the household and, therefore, to increase the number of vehicle-miles traveled. Secondly, older children may create additional travel demands than do younger children. In households with younger children, the number of vehicle-miles driven is similar to the number of miles driven in households with two or more adults with no children and in which the householder is younger than 60 . In fact, households with two adults or more registered the greatest 1988-to-1994 increase in the number of vehicle-miles traveled.

\section{Figure 3.5 Residential Vehicle-Miles Traveled per Household by Household Size and Survey Year, 1988 and 1994}

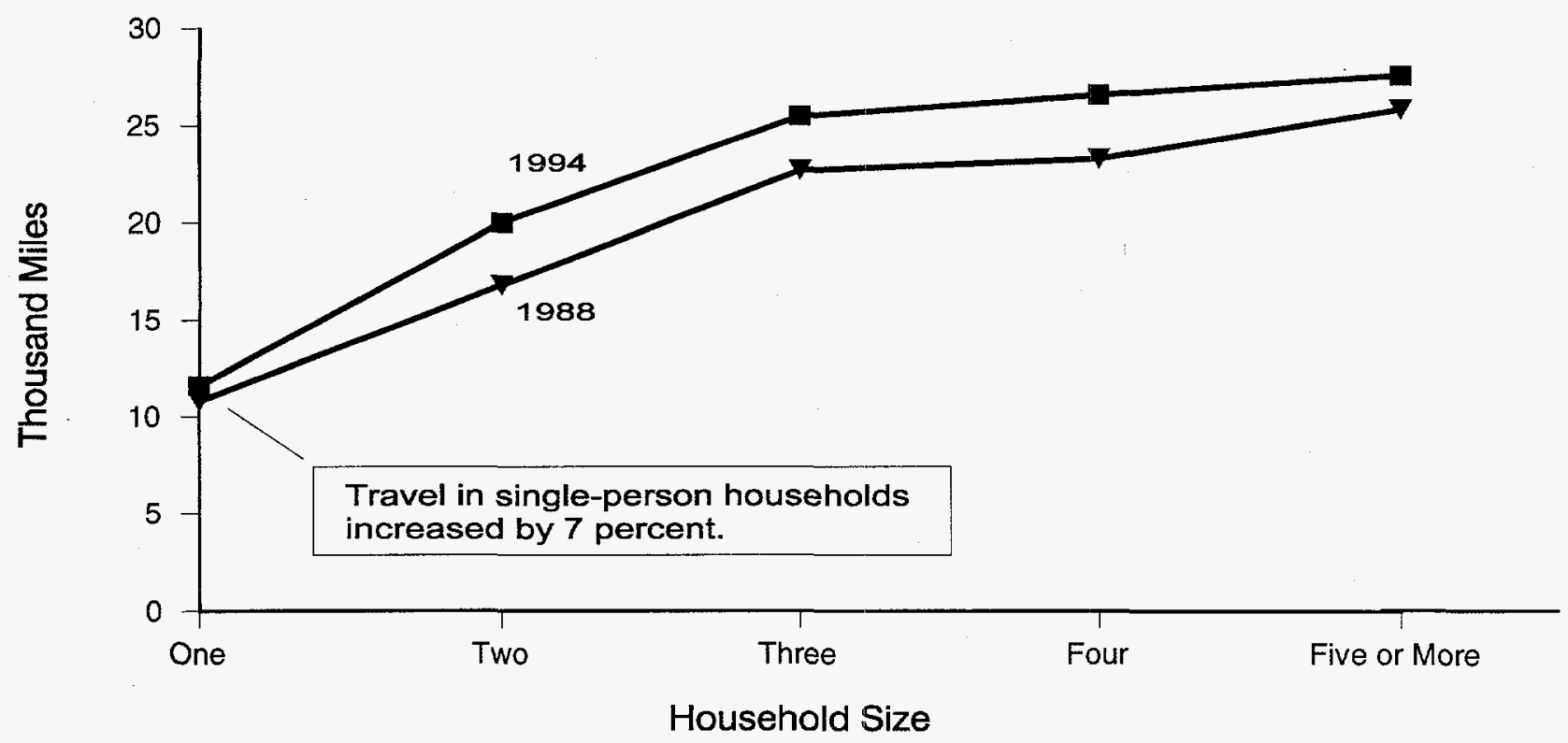

Sources: - 1988-Energy Information Administration, Household Vehicles Energy Consumption 1988, DOE/EIA-0464(88) (Washington, DC, February 1990), Table 7. $\quad$ 1994-Table 5.2 in this report. 

Number of Drivers and Survey Year, 1988 and 1994

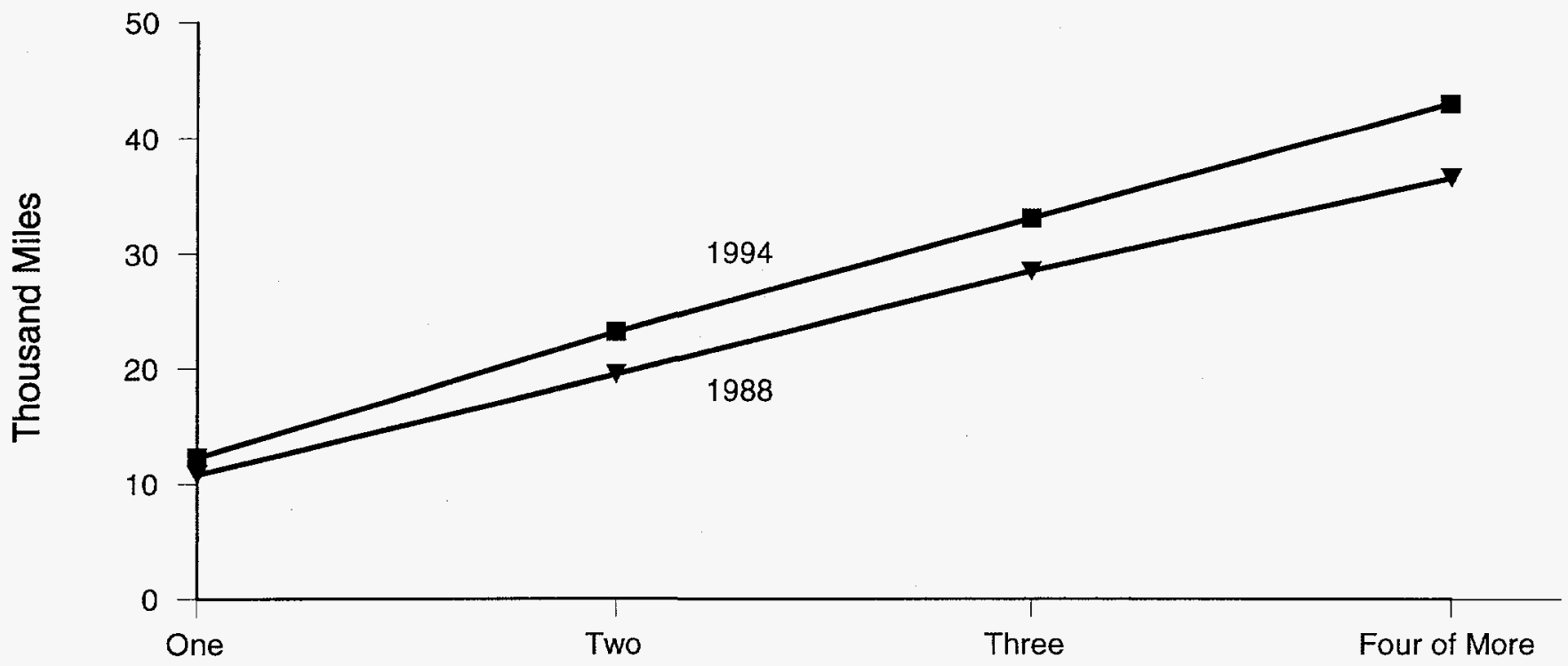

Number of Drivers

Sources: - 1988-Energy Information Administration, Household Vehicles Energy Consumption 1988, DOE/EIA-0464(88) (Washington, DC, February 1990), Table 7. $\bullet 1994-$ Table 5.2 in this report.

Figure 3.7 Residential Vehicle-Miles Traveled per Household by Household Composition and Survey Year, 1988 and 1994

Age in Years of Householder,

Households Without

Children, Two or

More Adults

Age in Years of Householder, Households Without Children, One Adult

Age in Years of Oldest Child, Households with Children
60 Years or More

35 to 59 Years

Under 35 Years

60 Years or More

35 to 59 Years

Under 35 Years

16 or 17 Years

7 to 15 Years

Under 7 Years

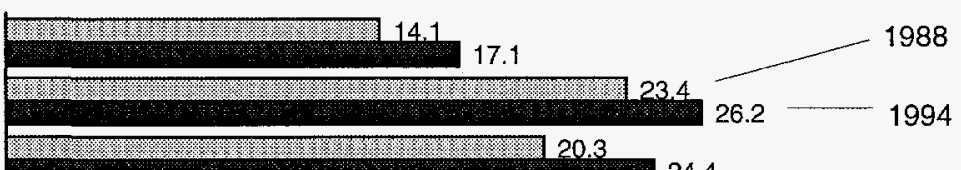

24.4

Households with children aged 16 or 17 drove the most.
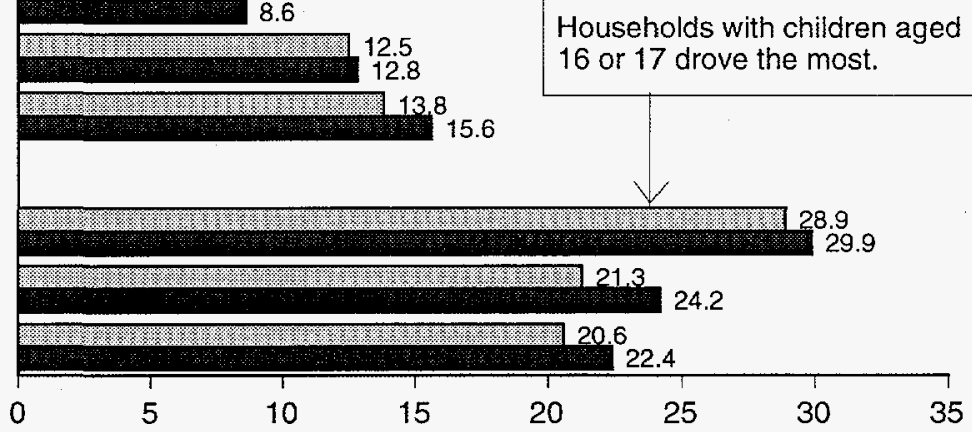

Thousand Miles

Note: Household composition refers to the number and ages of people in the household.

Sources: - 1988-Energy Information Administration, Household Vehicles Energy Consumption 1988, DOE/EIA-0464(88) (Washington, DC, February 1990), Table $14 . \quad$ 1994-Table 5.9 in this report. 


\section{Older Drivers Cover Fewer Miles}

Each residential vehicle has a primary driver. With the exception of 16- and 17-year-olds, primary drivers older than 49 generally drive their vehicles fewer miles than do primary drivers younger than 50 (Figure 3.8). After age 49, the older the primary driver, the fewer were the vehicle-miles traveled per year in general. As primary drivers, those 80 years and older averaged 6,100 miles per vehicle per year. The 16-year-old to 17-year-old age group averaged 9,600 vehicle-miles per year. Among the four age groups (drivers of age 18 through 49), the differences in number of vehicle-miles traveled per year were not statistically significant.

\section{Figure 3.8 Residential Vehicle-Miles Traveled per Vehicle by Age of Primary Driver,} 1994

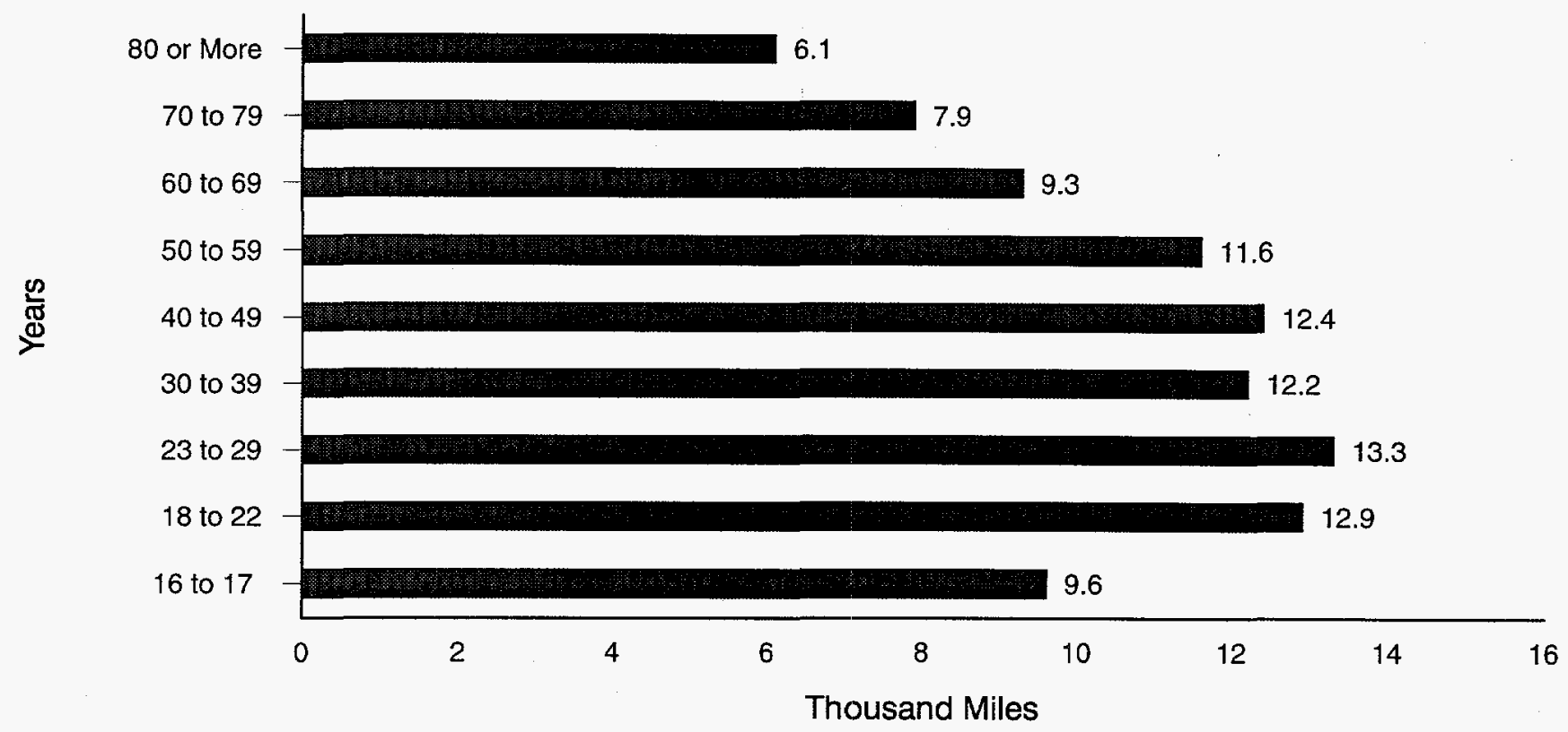

Source: Table 5.15 in this report.

\section{Higher Income Correlates with More Driving}

In general, higher income is correlated with more vehicle-miles traveled per household (Figure 3.9). Annual household income of $\$ 50,000$ or higher, when coupled with the presence of teenagers of driving age in the household, boosted average vehicle-miles traveled per household to 40,200 miles in 1994. And in households with that income and with two or more adults and no children, vehicle-miles traveled averaged nearly 27,400 . By comparison, in those households with annual income below $\$ 10,000$, vehicle-miles traveled averaged only 13,200 .

Household income does not correlate appreciably with the number of vehicle-miles traveled per vehicle. However, there is a correlation between household income and the number of vehicles per household (Figure 3.10). Highincome households tend to have more vehicles and thus a higher per-household average of vehicle-miles traveled. It is important to note that, in general, there is a positive correlation between higher household income, older age of head of household, a greater number of drivers, and an increased likelihood that the household will include children of driving age-all factors associated with a higher number of vehicle-miles per household. Households that include children 16 or 17 years old tend to have an older head of household. Older householders tend to have been in the work force longer, to have a correspondingly higher income, and to have more vehicles. For example, households with annual incomes of $\$ 50,000$ or higher have nearly twice as many vehicles as do households with annual incomes below $\$ 10,000$. 


\section{Figure 3.9 Residential Vehicle-Miles Traveled per Household by Annual Household Income, 1994}

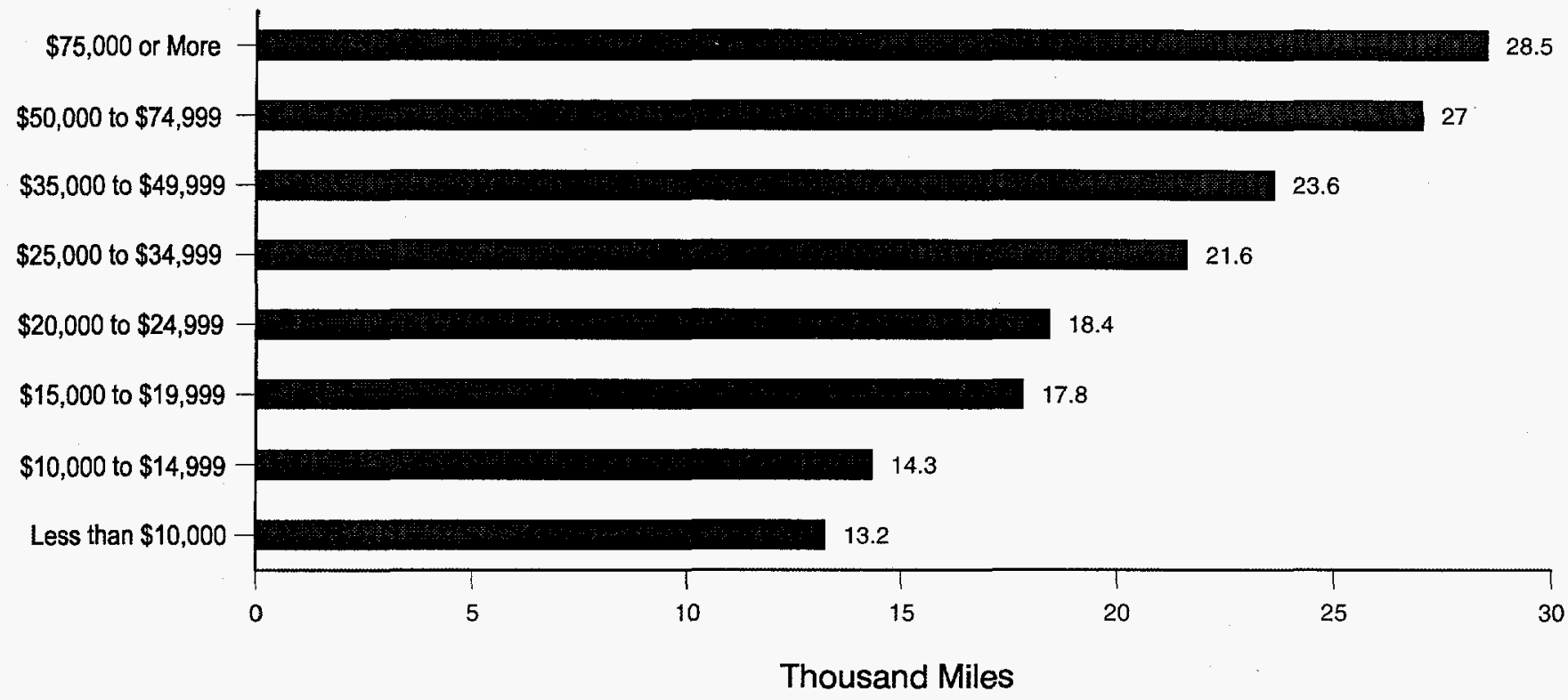

Source: Tables 5.2 in this report.

Figure 3.10 Average Number of Vehicles per Household by Household Income, 1994

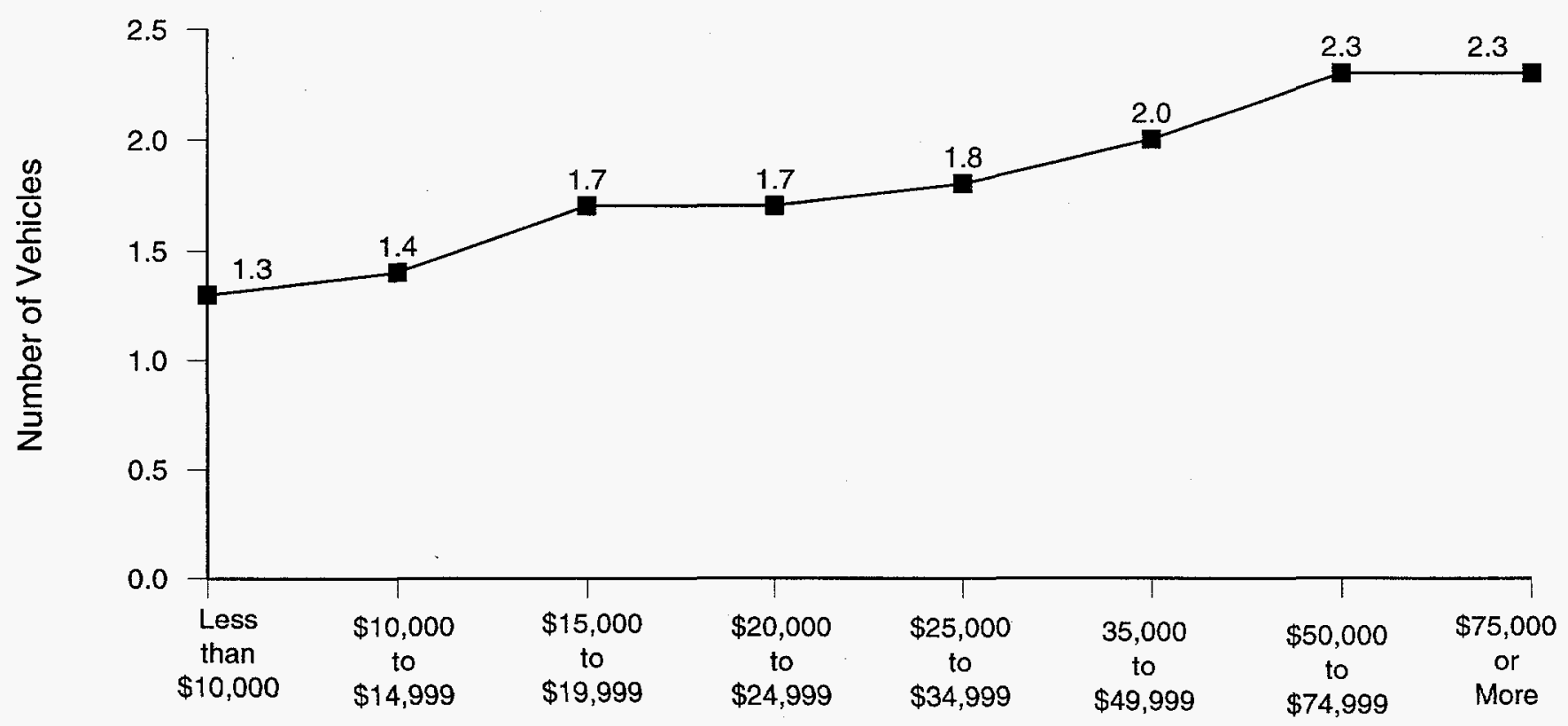

Source: Table 5.2 in this report. 



\section{Chapter 4. Fuel Economy, Consumption, and Expenditures}

This chapter analyzes trends in fuel economy, fuel consumption, and fuel expenditures, using data unique to the Residential Transportation Energy Consumption Survey, as well as selected data from other sources. Analysis topics include the following:

- Following the oil supply and price disruptions caused by the Arab oil embargo of 1973-1974, motor gasoline price increases, the introduction of corporate average fuel economy standards, and environmental quality initiatives helped to spur major changes in vehicle technology. But have the many advances in vehicle technology resulted in measurable gains in the fuel economy of the residential vehicle fleet?

- Despite national concerns about dependence on foreign oil and the deleterious effect on the environment of fossil fuel combustion, residential vehicle fleet fuel consumption was almost 8 billion gallons higher in 1994 than it had been in 1988. What factors led to the increase?

- Residential expenditures for energy averaged $\$ 2,571$ per household per year in $1994 .{ }^{16}$ How much of the total was attributed to residential vehicle fuel expenditures? And how did those expenditures compare with other vehicle-related operating expenses, such as insurance, financing, licensing, and depreciation?

\section{Fuel Economy Increased a Small Amount}

During the1983-to-1991 period, the overall fuel economy of the residential fleet increased by nearly 28 percent (about 3.5 percent per year), which helped to hold down total consumption. However, for the 1991-to-1994 period, the overall increase in fuel economy from 19.3 miles per gallon (mpg) to $19.8 \mathrm{mpg}$ was less than 3 percent (less than 1 percent per year). The large increase in the fleet fuel economy in the 1983-to-1991 period was due primarily to two factors: vehicles manufactured after 1982 had better fuel economy, and large numbers of pre-1983, lower fuel-economy vehicles were taken out of service. Each year, there are fewer pre-1983 vehicles to be replaced by newer vehicles, thus diminishing the opportunity for further increases in overall fleet fuel economy through replacement of older, lower fueleconomy vehicles with newer, higher fuel-economy vehicles.

The average fuel economy for the residential fleet was $19.8 \mathrm{mpg}$ in 1994, an 8-percent increase over 1988, when household vehicles averaged $18.3 \mathrm{mpg}$. Average fuel economy would have increased by a greater amount if all technological improvements had been devoted solely to that purpose rather than to increasing vehicle size and performance. Passenger cars, averaging $21.9 \mathrm{mpg}$, had the highest fuel economy. Large vans, averaging $13.8 \mathrm{mpg}$, exhibited the worst fuel economy.

\section{What is the Difference Between Fuel Economy and Fuel Efficiency?}

Fuel economy is a measure of how many miles a vehicle actually travels using a given amount of fuel. It is affected by factors such as the size of the engine and the shape and weight of the vehicle: For example, the heavier the Yehicle, the less fuel economic it will be, all other things being equal.

Fuel efficiency, as technically defined, is a straight-forward measure of how efficiently a vehicle engine processes motor fuel. A large, heavy car might have an engine that is as efficient as the engine in a small, light car, but it would probably have a lower fuel economy rating. 


\section{On Average, Newer Residential Vehicles Showed Little Improvement in Fuel Economy}

In general, fuel economy varies considerably by age and type of vehicle. However, there was relatively little variation for model years 1980 and later (Figure 4.1), which represented nearly 90 percent of all household vehicles. If all vehicles of model years 1979 and earlier were removed from the residential fleet, the overall fuel economy would only increase from $19.8 \mathrm{mpg}$ to $21.0 \mathrm{mpg}$. Any major increase in future years must therefore derive not from the retirement of older vehicles but from improvements in technology, and buying patterns of consumers.

\section{Figure 4.1 Average Fuel Economy of Residential Vehicles for Model Years} Through 1995

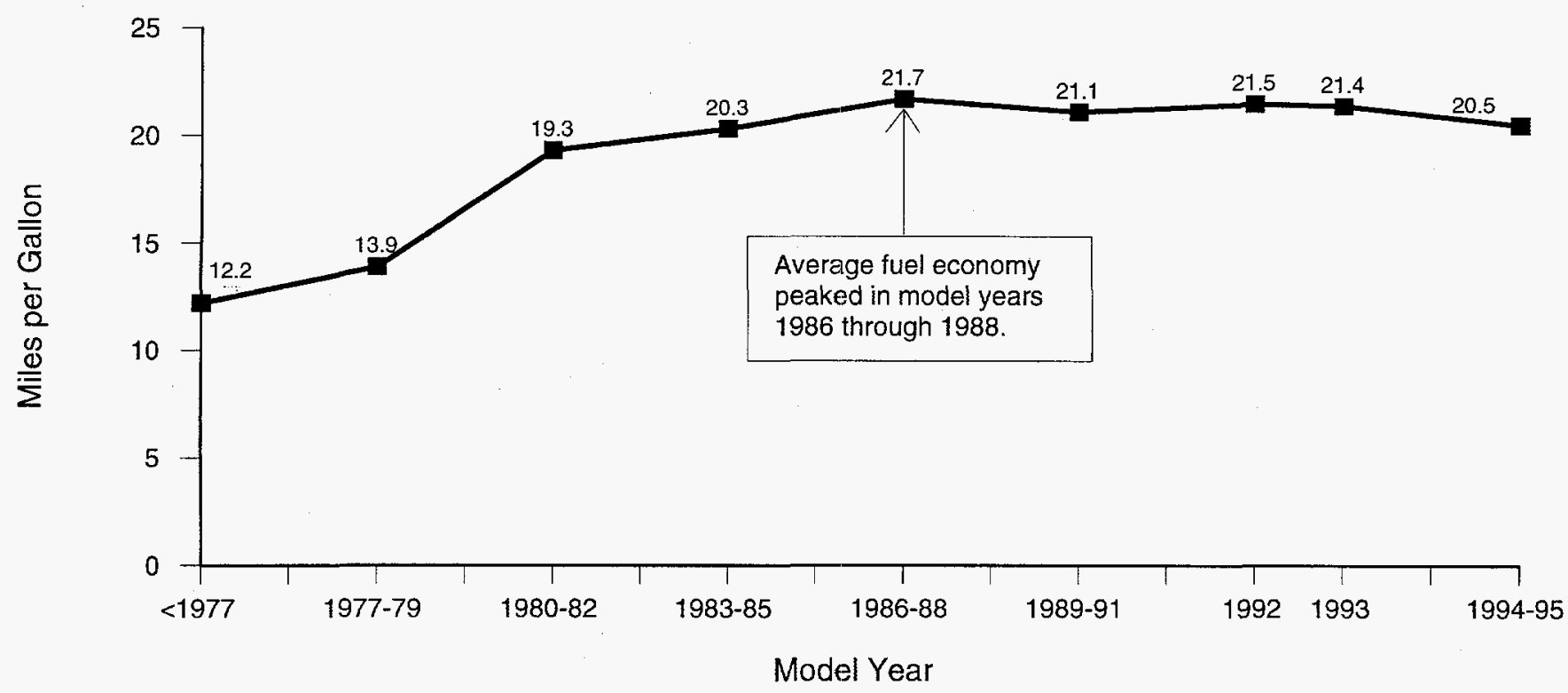

Source: Table 5.3 in this report.

\section{Federal Government Set Lower Fuel Economy Standards for Light Trucks}

One of the major factors affecting the average fuel economy is the composition of the vehicle fleet. New passenger cars in 1994 were subject to CAFE standards of $27.5 \mathrm{mpg}$ (unadjusted) and the overall passenger fleet averaged 21.9 mpg, compared with $19.7 \mathrm{mpg}$ in 1988 (Figure 4.2). New vehicles not classified as passenger cars-pickup trucks, sport-utility vehicles, minivans, and large vans, all classified as light-duty trucks - were subject to lower CAFE standards of $20.5 \mathrm{mpg}$ (unadjusted) and the overall fleet averaged $16.8 \mathrm{mpg}$ in 1994 . That average compares with 15.3 mpg in 1988. In 1994, large vans had the lowest mpg average of any category of light-duty truck and minivans had the highest. In addition to having lower CAFE standards, those vehicles were also exempt from the "gas guzzler" tax, which may have an effect on encouraging consumers to switch from larger, less fuel economic passenger cars to vehicles like minivans and sport utility vehicles which are even less fuel economic.

The number of vehicles getting less than 13 miles per gallon decreased rapidly from 1988 to 1994 (Figure 4.3). Residential vehicles getting 22 or more mpg increased by nearly 38 percent between 1988 and 1994 . The number of vehicles in the range between 13 and $21.9 \mathrm{mpg}$ increased, especially in the 16 to 18.9 miles per gallon category. Those intermediate ranges are where many new minivans, sport-utility vehicles, pickup trucks, and large vans would fall.

\section{Household Composition and Income Had Little Effect on Residential Vehicle Fuel Economy}

The fuel economy of vehicles in residential households showed little variation by household characteristics. Households without children did show an improvement in the fuel economy of their vehicles when the oldest adult was 
Figure 4.2 Average Fuel Economy of Residential Vehicles by Type of Vehicle, 1988 and 1994

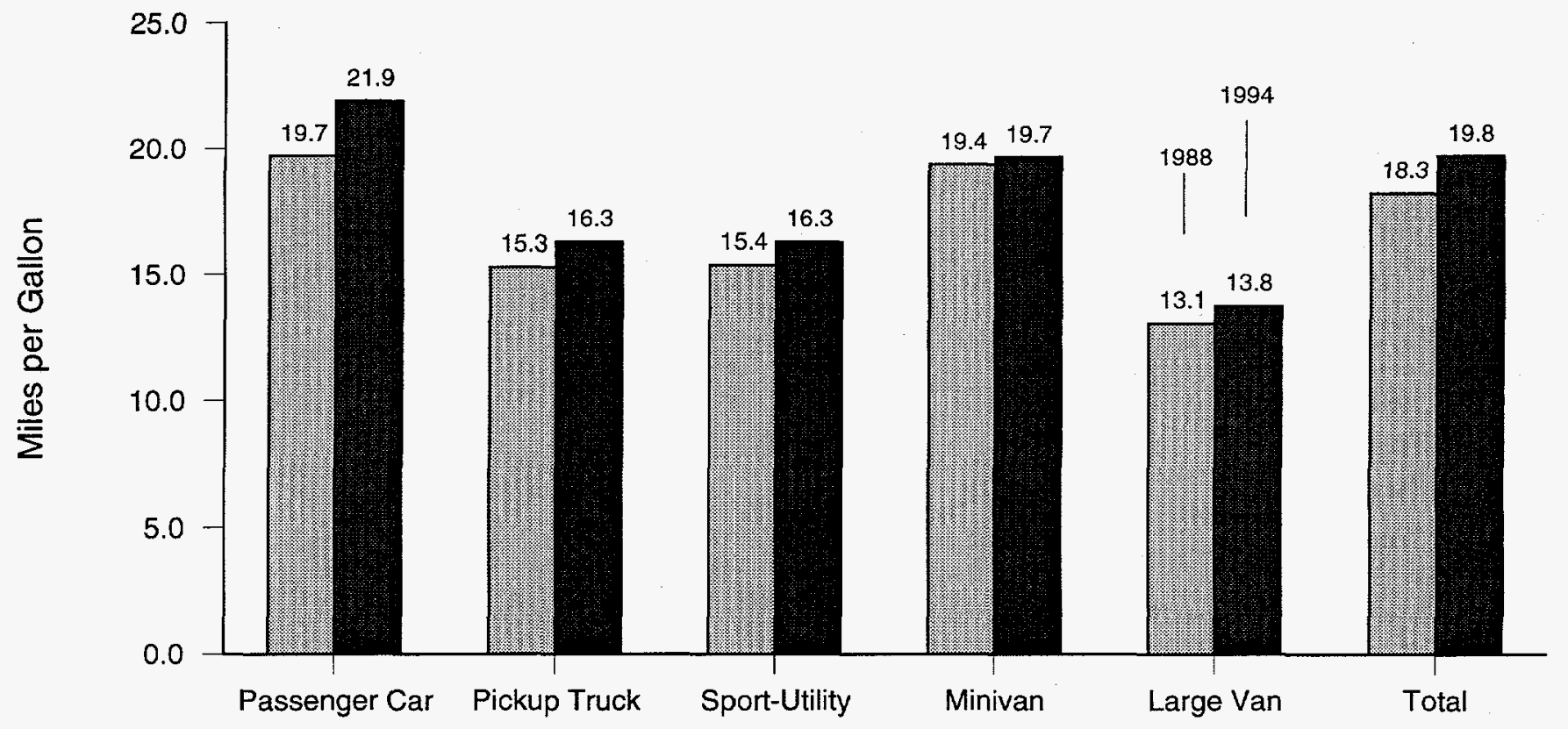

Sources: - 1988-Energy Information Administration, Household Vehicles Energy Consumption 1988, DOE/EIA-0464(88) (Washington, DC, February 1990), Table 8. 1994-Table 5.3 in this report.

\section{Figure 4.3 Number of Residential Vehicles by Fuel Economy, 1988 and 1994}

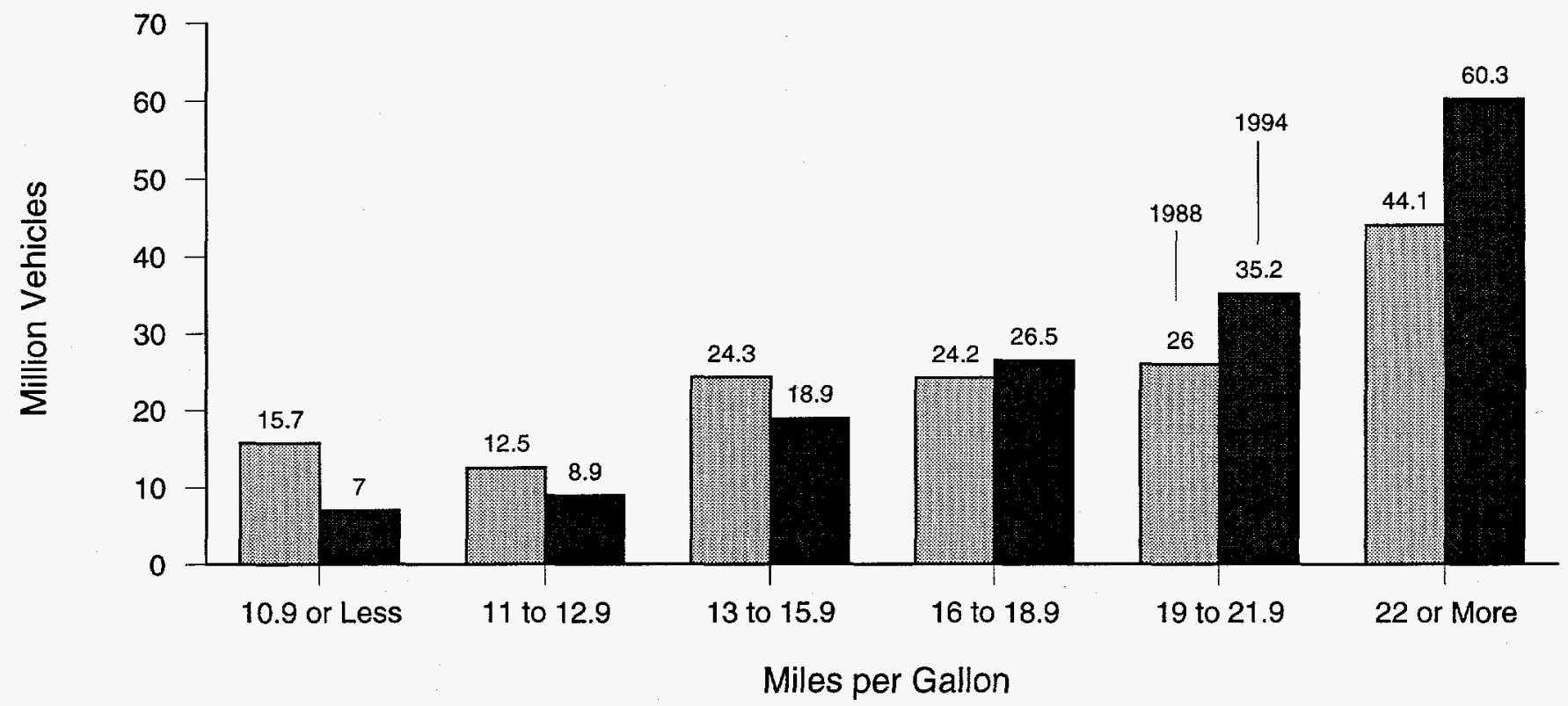

Sources - 1988-Energy Information Administration, Household Vehicles Energy Consumption 1988, DOE/EIA-0464(88) (Washington, DC, February 1990), Table $6 \bullet 1994-$ Table 5.1 in this report. 


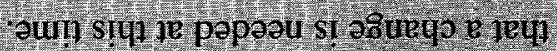

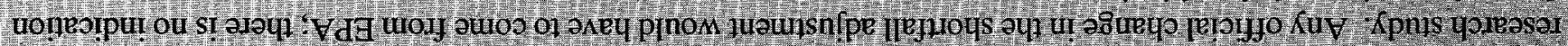

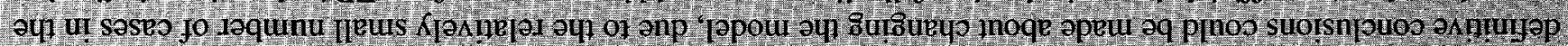

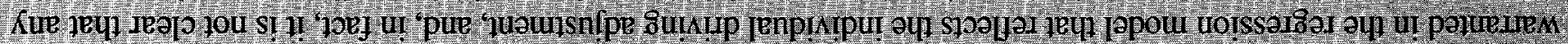

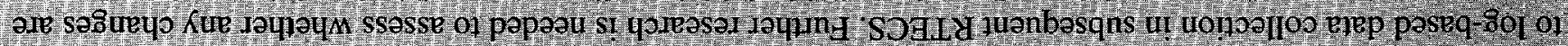

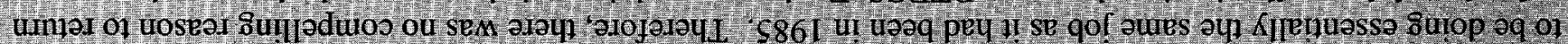

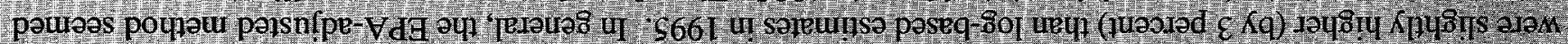
soneuinsa poseq- $V$ d

\section{s6uney va 01 alqeieduos ag ol panunuos s6o 7 lant}

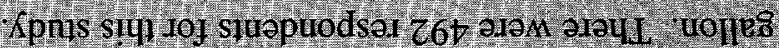

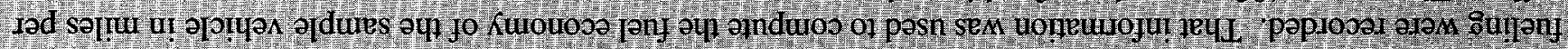

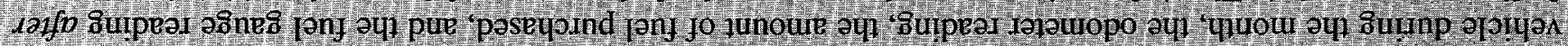

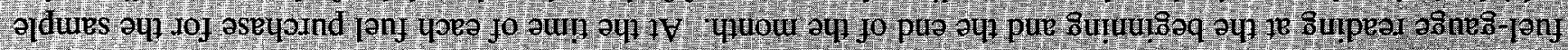

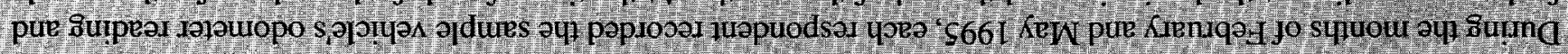

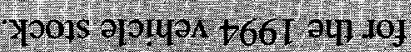

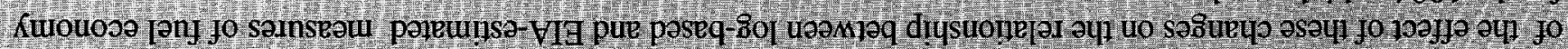

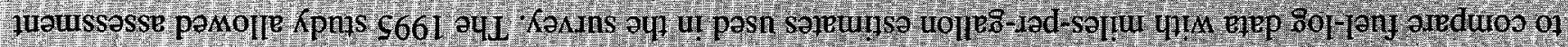

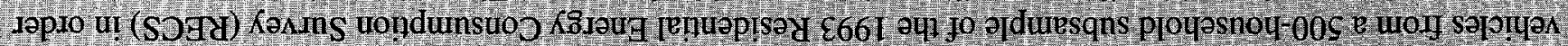

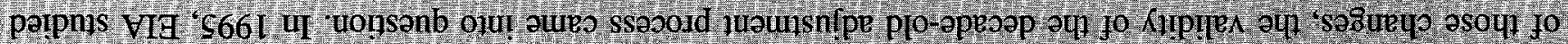

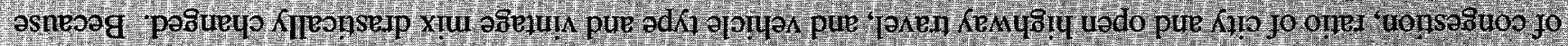

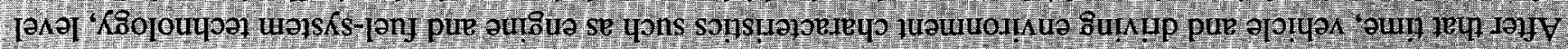

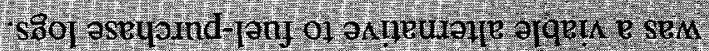

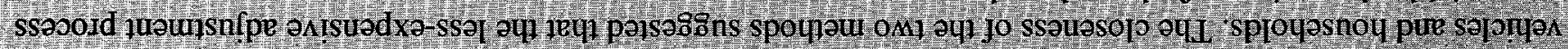

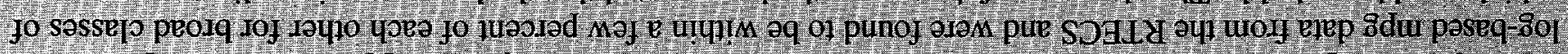

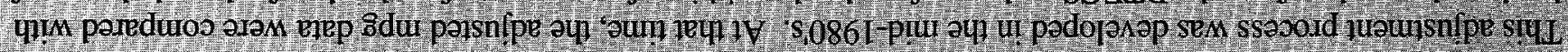

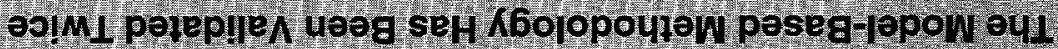

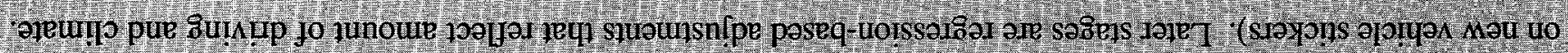

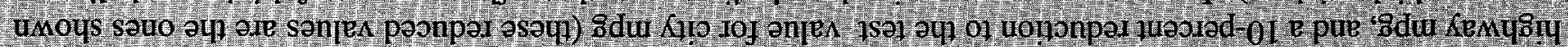

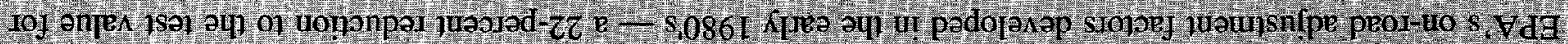

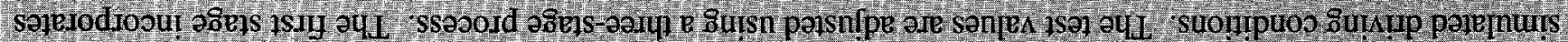

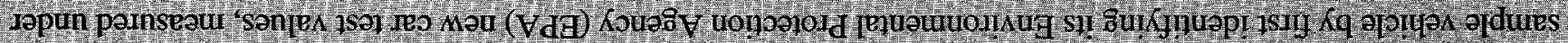

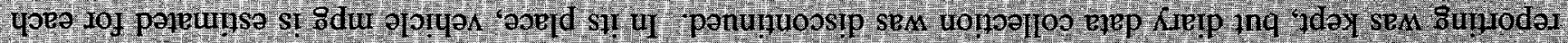

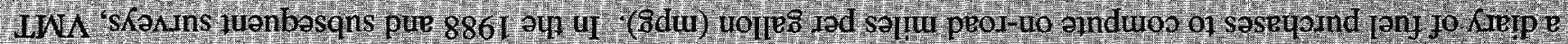

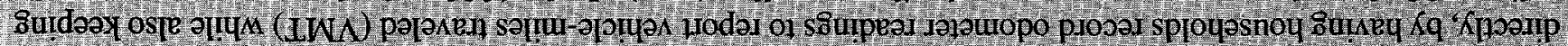

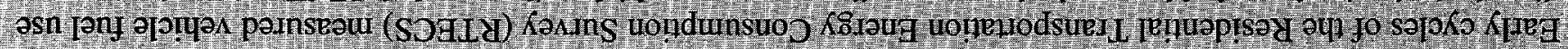

uopeuns 
under 35 years of age, as compared with households in which the oldest adult was 60 years old or more (Figure 4.4). Household income appears to have had even less effect on the fuel economy of vehicles in the household than did household composition. Interestingly (although not statistically significant), households with less than $\$ 5,000$ of annual income had the most fuel-economic vehicles (Figure 4.5).

\section{Figure 4.4 Average Fuel Economy of Residential Vehicles by Household Composition, 1994}

\begin{tabular}{|c|}
\hline $\begin{array}{l}\text { Age in Years of } \\
\text { Householder, } \\
\text { Households Without } \\
\text { Children, Two or } \\
\text { More Adults }\end{array}$ \\
\hline $\begin{array}{l}\text { Age in Years of } \\
\text { Householder, } \\
\text { Households Without } \\
\text { Children, One Adult }\end{array}$ \\
\hline $\begin{array}{l}\text { Age in Years of Oldest } \\
\text { Child, Households } \\
\text { With Children }\end{array}$ \\
\hline
\end{tabular}

Average of Households Without Children Average of Households With Children Average of All Households 35 to 59 Years

60 Years or More

16 or 17 Years 7 to 15 Years Under 7 Years

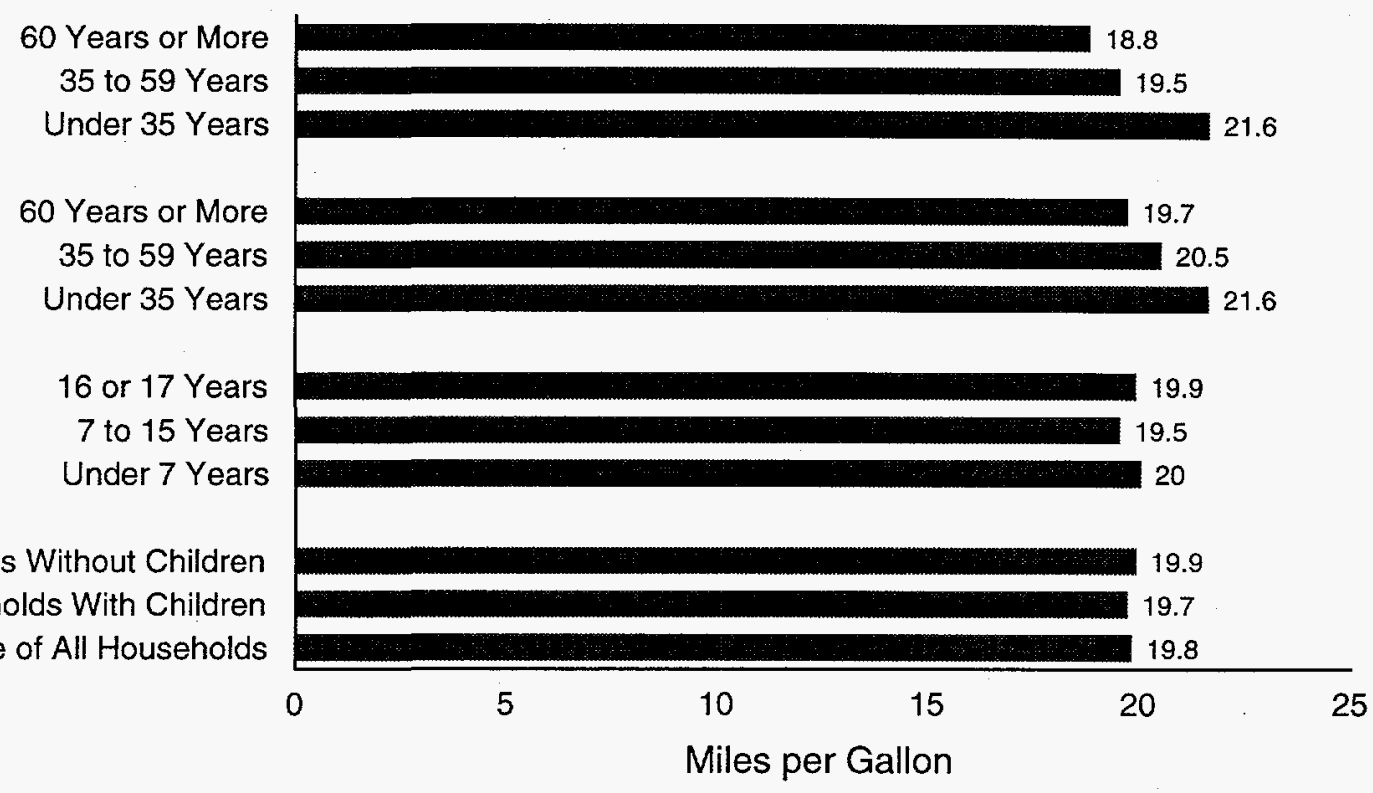

60 Years or More Under 35 Years 35 to 59 Years

Ner Gallon

Note: Household composition refers to the number and ages of people in the household.

Source: Table 5.3 in this report.

\section{Figure 4.5 Average Fuel Economy of Residential Vehicles by Annual Household Income, 1994}

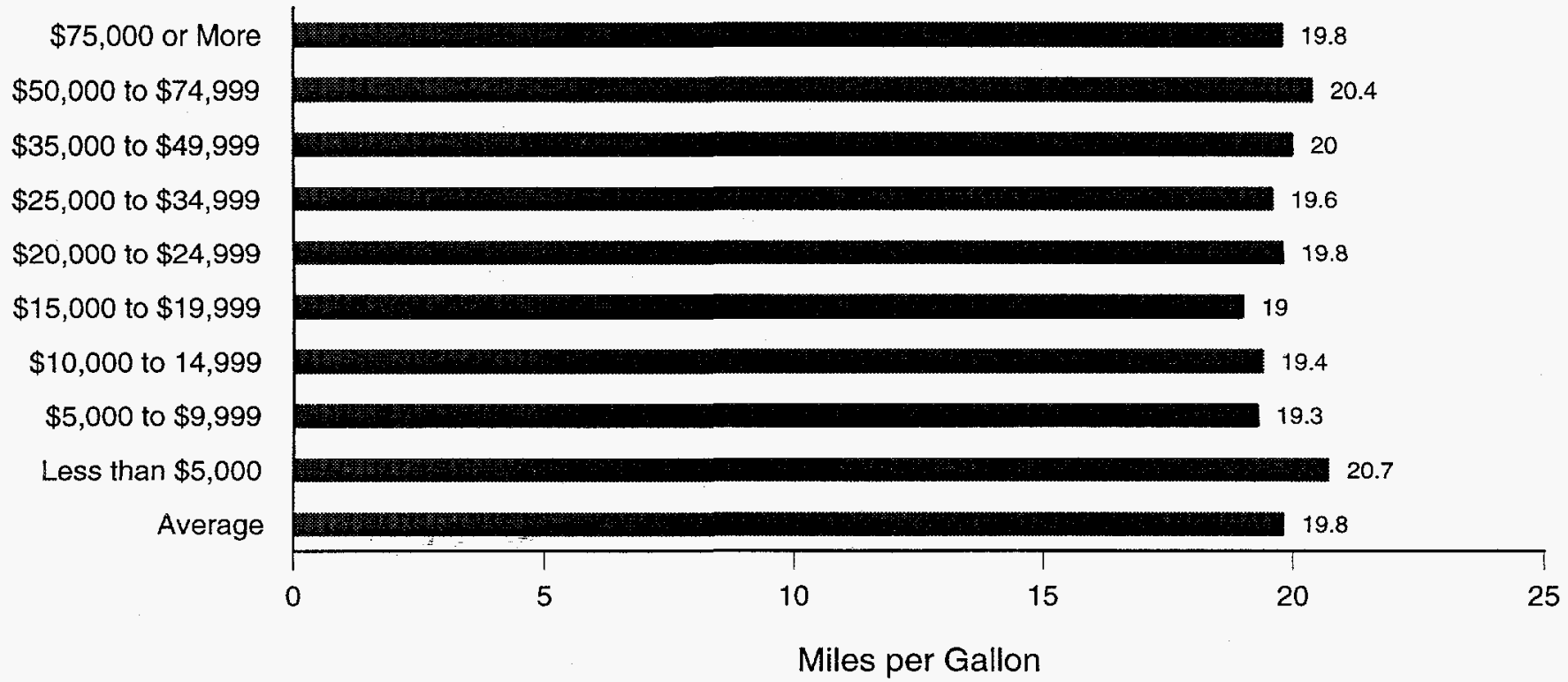

Sources: Table 5.3 in this report. 


\section{Fuel Consumption Increased in 1994}

In 1991, 82.8 billion gallons of motor fuel were consumed by the passenger cars, minivans, sport-utility vehicles, pickup trucks, and large vans that comprise the residential vehicle fleet (Figure 4.6). That level of consumption was not significantly different from the 1988 level of 82.4 billion gallons. But in 1994, consumption totaled 90.6 billion gallons, a 9-percent increase. The nearly 8-billion-gallon increase was roughly the equivalent of motor fuel imports of half a million barrels per day for a year.

The number of vehicle-miles traveled showed steady growth during the 1991-to-1994 period. One reason that total consumption rose more rapidly than the number of miles traveled during the period was the slowdown in the increase in the fuel economy of the residential fleet.

\section{Passenger Cars Consumed the Largest Share of Motor Fuel}

Although passenger cars still accounted for most of the motor fuel consumed by residential vehicles, their share of the total declined from nearly 70 percent in 1988 to 60 percent in 1994 (Figure 4.7). The decrease came as a result of growing consumer preferences for minivans, pickup trucks, and sport-utility vehicles. In the future, as light trucks continue to increase as a percentage of the residential fleet, their higher number of vehicle-miles traveled and lower fuel economies will accelerate the total amount of fuel they consume.

\section{Figure 4.6 Total Residential Vehicle Fuel Consumption, 1983, 1985, 1988, 1991 , and 1994}

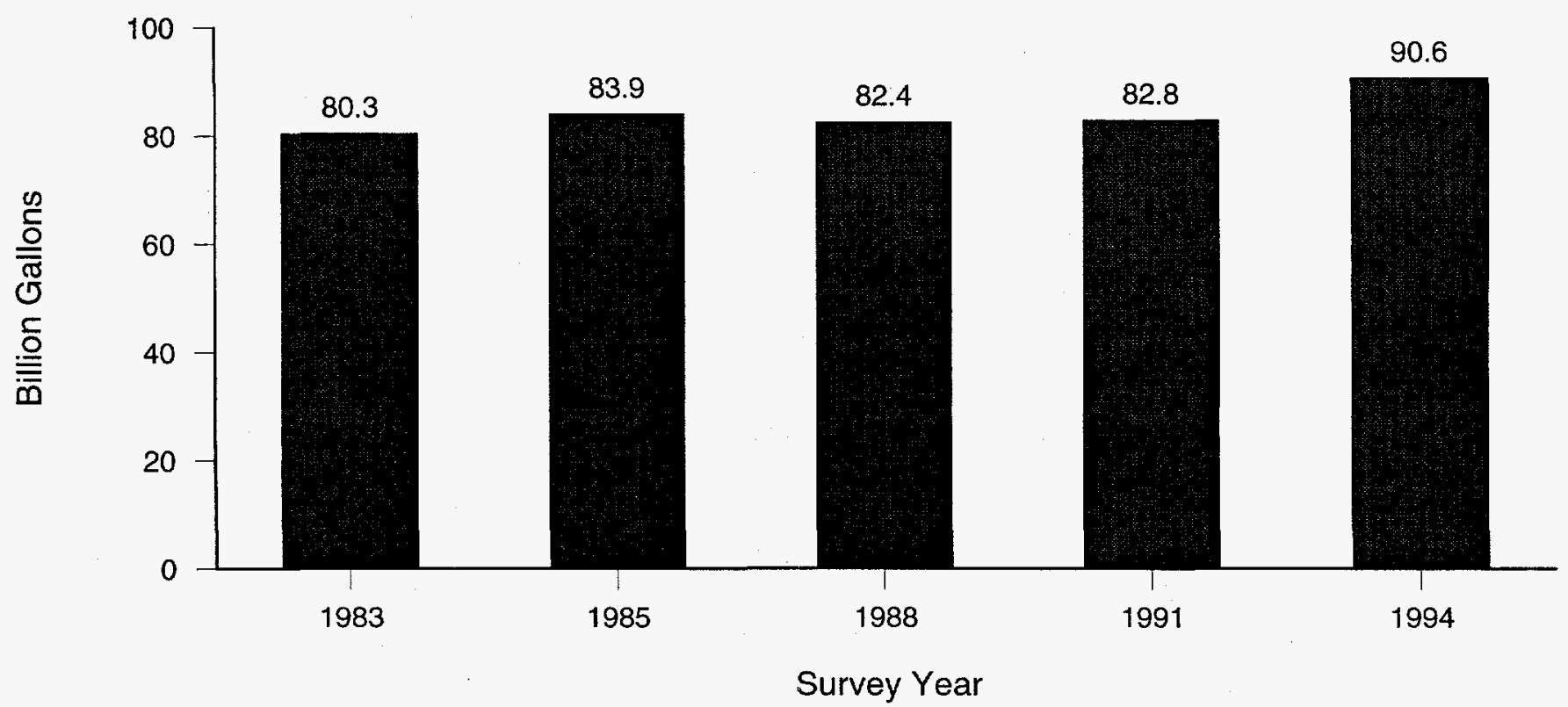

Sources: • 1983-Energy Information Administration (EIA), Consumption Patterns of Household Vehicles 1983, DOE/EIA0464(83) (Washington, DC, January 1985), Table 11. 1985-EIA, Consumption Patterns of Household Vehicles 1985, DOE/EIA-0464(85) (Washington, DC, April 1987), Table 8. • 1988-EIA, Household Vehicles Energy Consumption 1988, DOE/EIA-0464(88) (Washington, DC, February 1990), Table 6. 1991-ElA, Household Vehicles Energy Consumption 1991, DOE/EIA-0464(91) (Washington, DC, December 1993), Table 8. • 1994-Table 5.1 in this report. 


\section{Figure 4.7 Total Residential Vehicle Fuel Consumption by Type of Vehicle, 1988 and 1994}

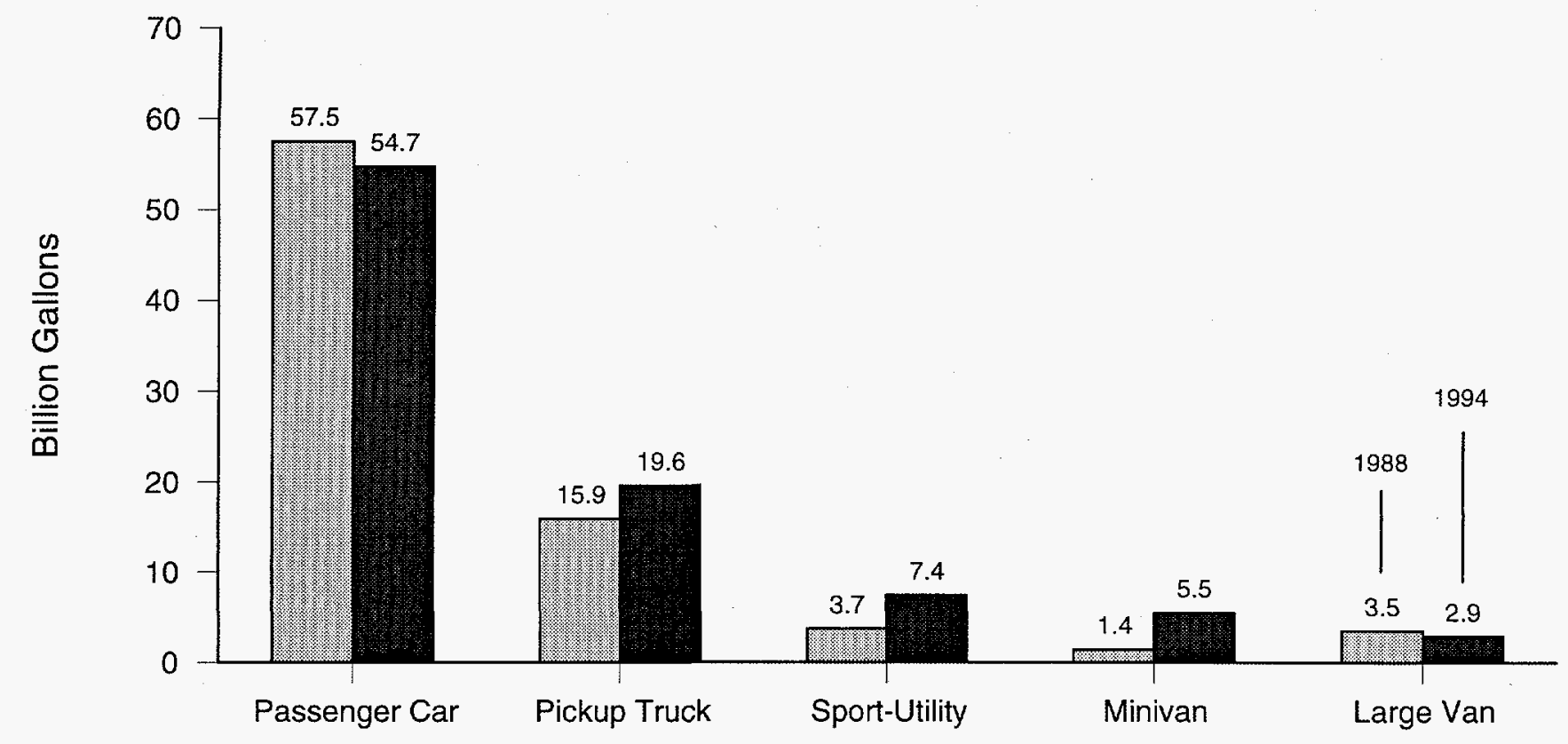

Sources: •-1988 Energy Information Administration, Household Vehicles Energy Consumption 1988, DOE/EIA-0464(88) (Washington, DC, February 1990), Table 6. • 1994-Table 5.1 in this report.

\section{Passenger Cars Averaged the Least Motor Fuel Consumption per Vehicle}

At 514 gallons per year, passenger cars registered the smallest fuel consumption per vehicle of all vehicle types in the residential fleet (Figure 4.8). That amount was similar to the 526-gallon average reported in 1988. Light-duty residential trucks (that is, all residential vehicles other than passenger cars) showed an increase in average consumption from 652 gallons per year in 1988 to 712 gallons per year in 1994. Fuel consumption by pickup trucks, the most prevalent of light trucks in the residential fleet, increased nearly 11 percent.

\section{The Newest Vehicles Consumed the Most Fuel Because They Were Driven More}

The newest vehicles in 1994 (model years 1993 and later) were driven more than 60 percent farther than the oldest vehicles (model years 1979 and earlier). Nevertheless, the oldest vehicles, because of their lower fuel economies, consumed nearly as much fuel on average as did the newest vehicles. Motor fuel consumption by vehicles of model years 1994 and 1995 averaged 697 gallons per year in 1994 (Figure 4.9). By comparison, vehicles of model years 1980 through 1982 consumed an average of 510 gallons per year in 1994. Although, as would be expected, the number of vehicles older than model year 1980 decreases over time, in 1994 there were still about 20 million of them in the residential fleet.

\section{Vehicles in the South Consumed the Most Fuel}

Average fuel consumption per vehicle did not vary much by region (Figure 4.10). Vehicles in the South consumed an average of 598 gallons per year. Vehicles in the Midwest and West averaged 580 gallons per year and 568 gallons per year, respectively. In the Northeast, vehicles consumed 545 gallons per year, the lowest average of the four regions. 
Figure 4.8 Average Residential Vehicle Fuel Consumption by Type of Vehicle, 1988 and 1994

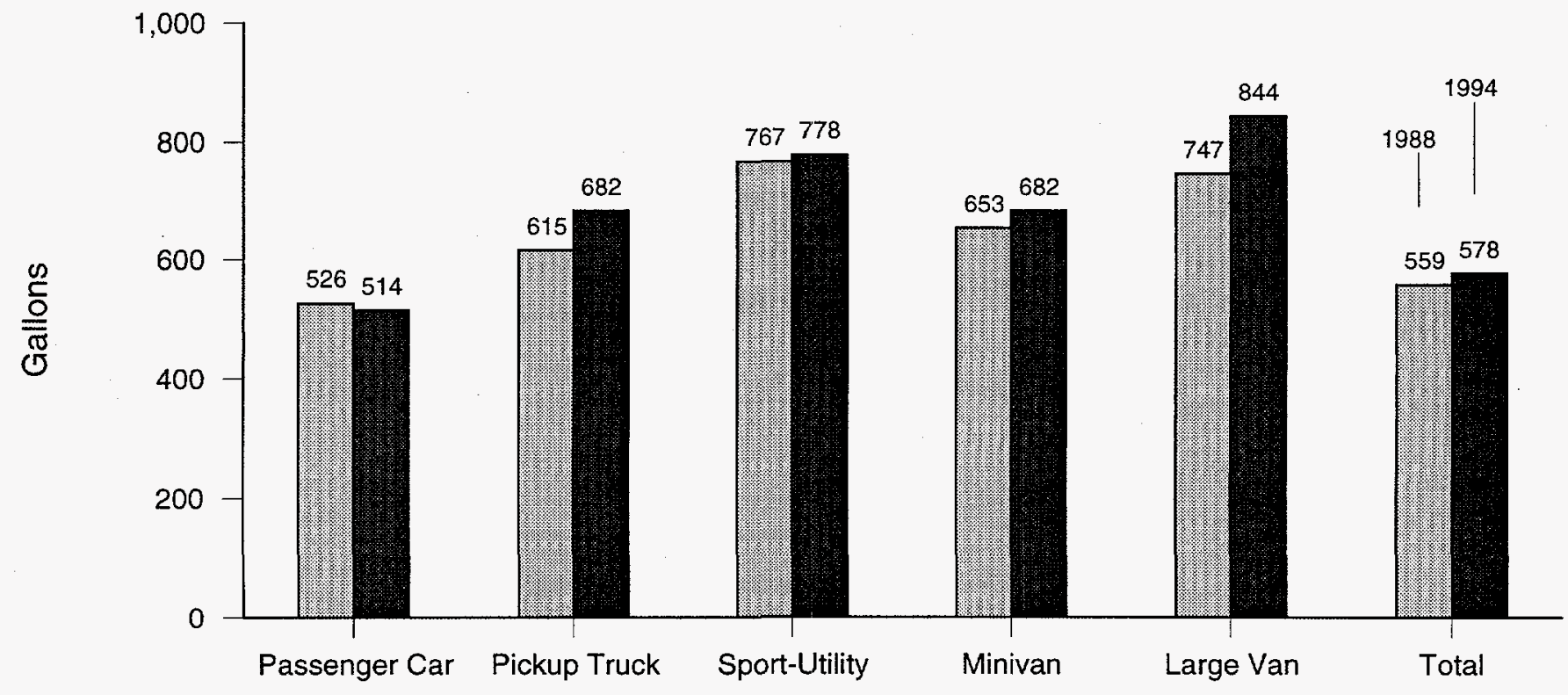

Sources: - -1988 Energy Information Administration, Household Vehicles Energy Consumption 1988, DOE/EIA-0464(88) (Washington, DC, February 1990), Table 8. $\bullet 1994-$ Table 5.3 in this report.

Figure 4.9 Average Residential Vehicle Fuel Consumption per Vehicle for Model Years Through 1995

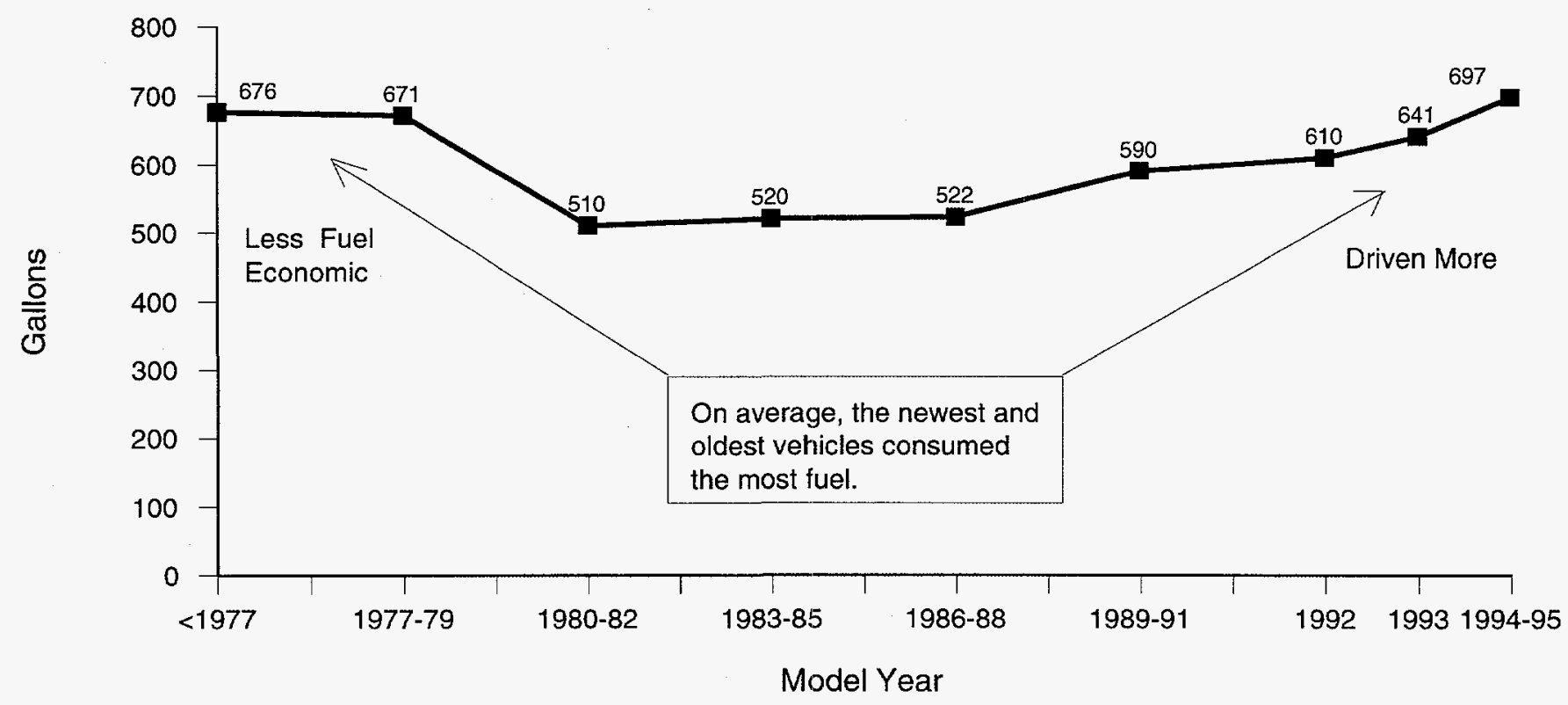

Source: Table 5.3 in this report. 


\section{Figure 4.10 Average Annual Residential Vehicle Fuel Consumption by Region, 1994}
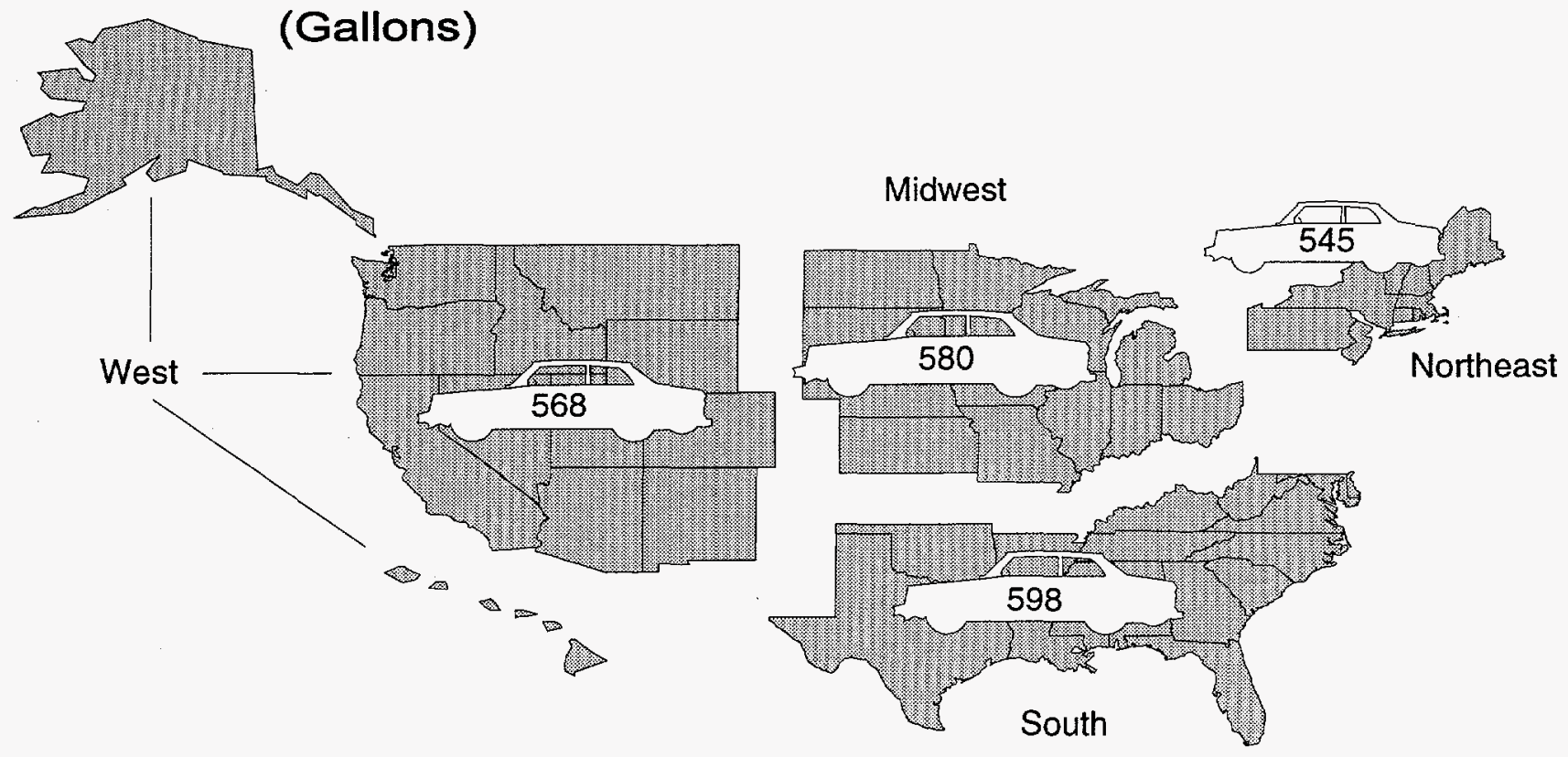

Source: Table 5.3 in this report.

\section{Fuel Expenditures Varied by Household Characteristics}

In households reporting owning or having access to at least one vehicle in 1994, residential expenditures for energy for transportation averaged $\$ 1,234$. Which households were likely to spend more than the average? And how did average residential expenditures compare with residential expenditures for energy for all other uses, including space heating?

\section{Inflation-Adjusted Expenditures for Vehicle Fuel Varied Very Little Over Time}

The inflation-adjusted cost of purchasing fuel for residential vehicles remained relatively constant from 1988 through 1994 (Figure 4.11). During that period, the average fuel consumption per vehicle also changed little. The improvement in fuel economy for the residential vehicle fleet helped to keep expenditures and consumption nearly unchanged.

\section{Household Composition and Income Affected Fuel Expenditures}

Household composition influenced motor fuel expenditures and average fuel expenditures per household varied widely. Households with a single adult aged 60 years old or more spent on average only $\$ 498$ per year, while households with children aged 16 or 17 years spent on average $\$ 1,727$ per year (Figure 4.12 ).

Fuel expenditures per household also varied by household income. In general, higher-income households had higher expenditures for vehicle fuel (Figure 4.13). For example, householders with annual incomes of $\$ 75,000$ or more 
Figure 4.11 Average Fuel Expenditures per Residential Vehicle by Survey Year, $1980,1983,1985,1988,1991$, and 1994

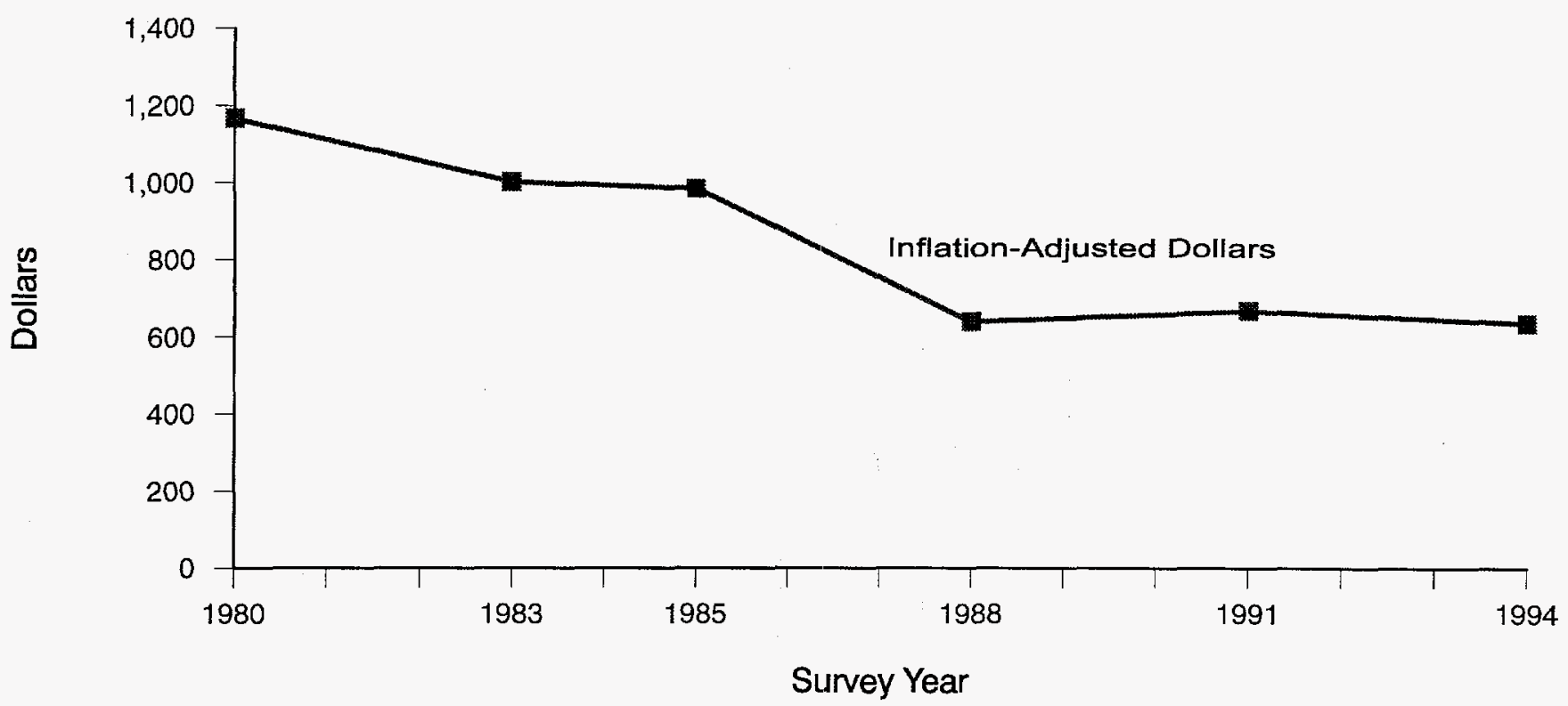

Sources: Fuel Expenditures: - 1983-Energy Information Administration (EIA), Consumption Patterns of Household Vehicles 1983, DOE/EIA-0464(83) (Washington, DC, January 1985), Table 7. - 1985-EIA, Consumption Patterns of Household Vehicles 1985, DOE/EIA-0464(85) (Washington, DC, April 1987), Table 8. - 1988-EIA, Household Vehicles Energy Consumption 1988, DOE/EIA-0464(88) (Washington, DC, February 1990), Table 8. - 1991-EIA, Household Vehicles Energy Consumption 1991, DOE/EIA-0464(91) (Washington, DC, December 1993), Table 10. $1994-$ Table 5.3 in this report. Implicit Price Deflators: EIA, Annual Energy Review 1995, DOE/EIA-0384(95) (Washington, DC, July 1996), Table E1.

\section{Figure 4.12 Average Vehicle Fuel Expenditures per Household, 1994}

\begin{tabular}{|c|}
\hline $\begin{array}{l}\text { Age in Years of } \\
\text { Householder, } \\
\text { Households Without } \\
\text { Children, Two or } \\
\text { More Adults }\end{array}$ \\
\hline $\begin{array}{l}\text { Age in Years of } \\
\text { Householder, } \\
\text { Households Without } \\
\text { Children, One Adult }\end{array}$ \\
\hline $\begin{array}{l}\text { Age in Years of Oldest } \\
\text { Child, Households } \\
\text { With Children }\end{array}$ \\
\hline
\end{tabular}

Average of Households Without Children Average of Households With Children Average of All Households 60 Years or More
35 to 59 Years
Under 35 Years

60 Years or More 35 to 59 Years Under 35 Years

16 or 17 Years 7 to 15 Years Under 7 Years

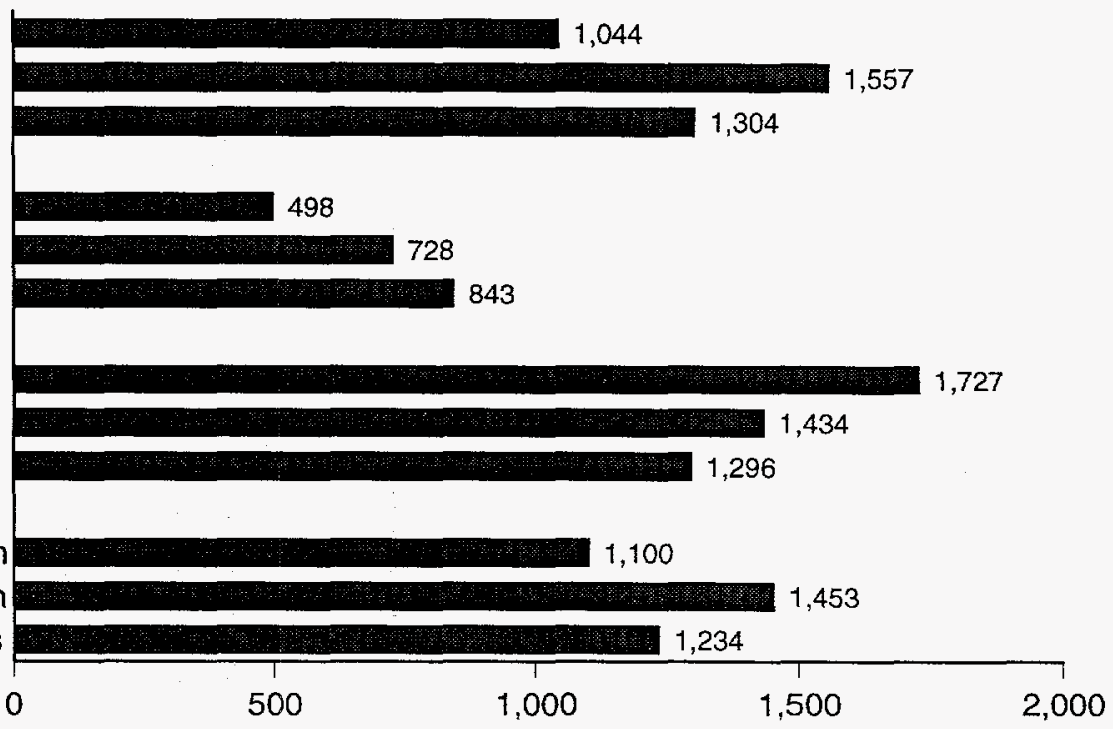

Dollars

Source: Table 5.2 in this report. 
reported expenditures of $\$ 1,692$ per year, whereas the poorest households (those with incomes of less than $\$ 5,000$ ) reported expenditures of $\$ 923$. Energy expenditures for residential transportation were close to half of the average household's total energy expenditures. Expenditures for non-transportation-related energy needs, such as heating, airconditioning, lighting, and cooking, were $\$ 1,337$, only slightly higher than the $\$ 1,234$ spent for vehicle fuel.

\section{Figure 4.13 Average Vehicle Fuel Expenditures and Household Energy Expenditures by Household Income, 1994}

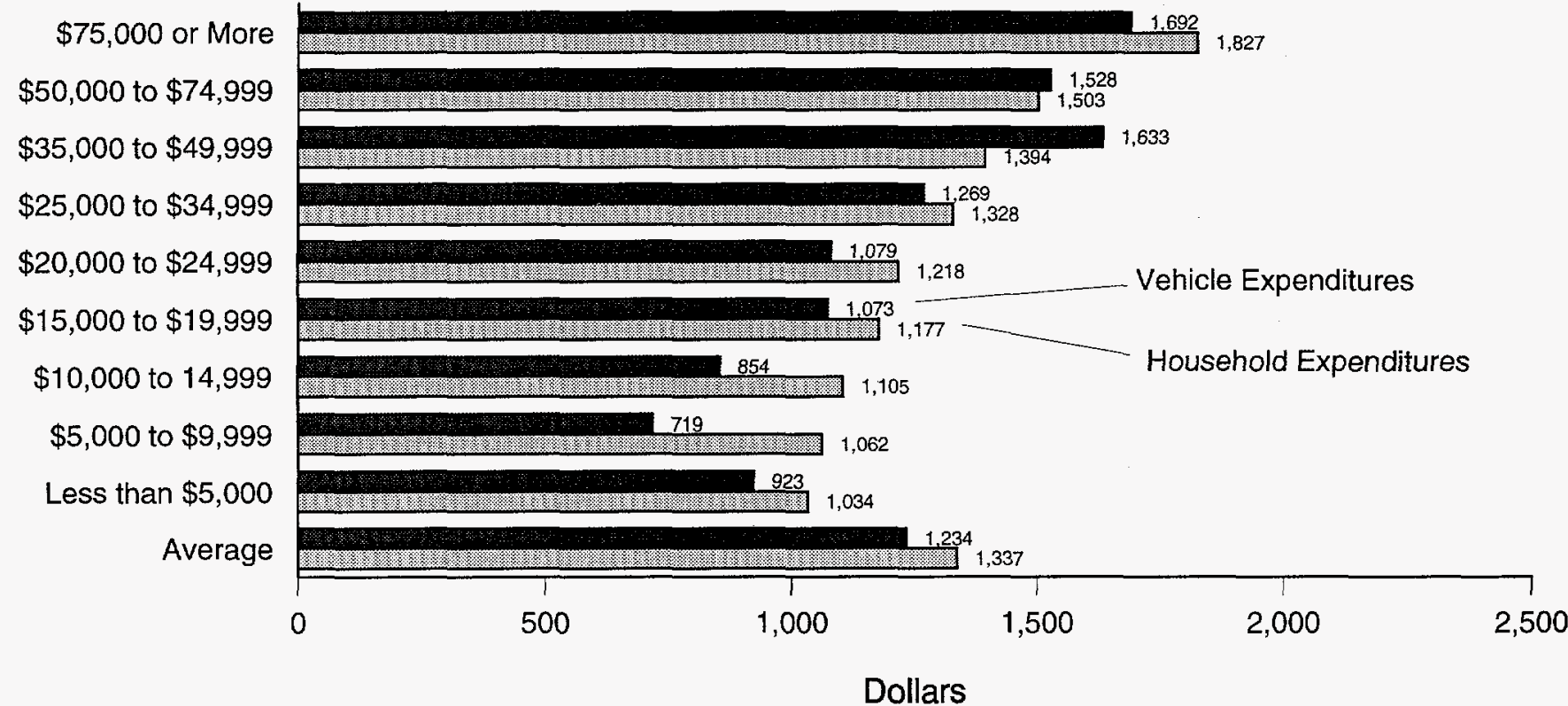

Note: Household energy expenditures exclude household vehicle fuel expenditures.

Source: Table 5.18 in this report. 



\section{Text Notes and Sources}

\section{Chapter 1}

1. U.S. Department of Transportation, Summary of Fuel Economy Performance, April 1996, (Washington, DC, April 1996), p. 4.

2. EIA conducts numerous energy-related surveys. In general, the surveys can be divided into two broad groups: supply surveys, directed to the suppliers and marketers of specific energy sources, that measure the quantities of specific fuels produced for and/or supplied to the market; and consumption surveys, which gather information on the types of energy used by the end users along with the characteristics of those end users that are associated with energy use. The RTECS belongs to the consumption group because it collects information directly from the end user, the household.

\section{Chapter 2}

3. Energy Information Administration, Annual Energy Review 1995, DOE/EIA-0384(95) (Washington, DC, July 1996), Table 1.5.

4. 1988-Federal Highway Administration (FHA), Highway Statistics 1988, FHWA-PL-89-003 (Washington, DC, September 1989), Table DL-1B. 1994 -FHA, Highway Statistics 1994, FHWA-PL-95-042 (Washington, DC, October 1995), Table DL-1B.

5. "Chained dollars" is a measure used to express real prices. Real prices are those that have been adjusted to remove the effect of changes in the purchasing power of the dollar; they usually reflect buying power relative to a reference year (in this case, 1992). Prior to 1996, real prices were expressed in constant dollars, a measure based on the weights of goods and services in a single year, usually a recent year. In 1996, the U.S. Department of Commerce introduced the chained-dollar measure. The new measure is based on the average weights of goods and services in successive pairs of years. It is "chained" because the second year in each pair, with its weights, becomes the first year of the next pair. The advantage of using the chained-dollar measure is that it is more closely related to any given period covered and is therefore subject to less distortion over time.

6. Energy Information Administration, Annual Energy Review 1995, DOE/EIA-0384(95) (Washington, DC, July 1996), Table 5.21.

7. Energy Information Administration, Household Energy Consumption and Expenditures 1993, DOE/EIA-0321(93) (Washington, DC, October 1995), p. 296.

8. Energy Information Administration, Annual Energy Review 1995, DOE/EIA-0384(95) (Washington, DC, July 1996), Table 5.12b.

9. International Trade Association, "Corporate Average Fuel Economy Explained." Online. (http://www.ita.doc.gov/industry/basic/cafe.html) (March 5, 1997).

10. Environmental Protection Agency, Light-Duty Automotive Technology and Fuel Economy Trends Through 1996, EPA/AA/TDSG/96-01 (Washington, DC, August 1996), Table 1.

11. Environmental Protection Agency, Light-Duty Automotive Technology and Fuel Economy Trends Through 1996, EPA/AA/TDSG/96-01 (Washington, DC, August 1996), Table 1. 


\section{Chapter 3}

12. 1988-Federal Highway Administration (FHA), Highway Statistics 1988, FHWA-PL-98-003 (Washington, DC, September 1989), Table DL-1B. 1994 -FHA, Highway Statistics 1994, FHWA-PL-95-042 (Washington, DC, October 1995), Table DL-1B.

13. The 1994 residential vehicle fleet included a small number of 1995 model year vehicles.

14. Energy Information Administration calculation based on mileage from New York City to San Francisco of 2,946, as cited in Rand McNally \& Company, Rand McNally Motor Carriers' Road Atlas 1991, p. 148.

15. The 1993 household data used in this report are collected by the Residential Energy Consumption Survey, another end-use consumption survey conducted by the Energy Information Administration.

\section{Chapter 4}

16. Table 5.18 in this report. 


\section{Chapter 5. Detailed Tables}

The following tables present detailed characteristics of vehicles in the residential sector. Data are from the 1994 Residential Transportation Energy Consumption Survey.

\section{Table Organization}

The "Detailed Tables" section consists of three types of tables: (1) Tables of totals such as number of vehicle-miles traveled (VMT) or gallons consumed; (2) tables of per household statistics such as VMT per household; and (3) tables of per-vehicle statistics, such as vehicle fuel consumption per vehicle. The tables have been grouped together by specific topics such as model-year data or family-income data to facilitate finding related information. The QuickReference Guide to the detailed tables indicates major topics of each table.

\section{Quick-Reference Guide}

Topic

Summary

Totals

Per Household

Per Vehicle

Vehicle Model Year

Total Vehicles

Miles per Gallon

Gallons per Vehicle

Family Income

Total Miles Traveled

Total Gallons

Miles per Household

Gallons per Household

Household Composition

Total Vehicles

Miles per Household

Miles per Gallon

Per Vehicle

Vehicle Type

Total Gallons

Miles per Vehicle

Total Vehicles

Table Number

5.1

5.2

5.3

5.4

5.5

5.6

5.7

5.8

5.9

5.10

5.12

5.14

5.15

5.16

Number of Households

Household and Vehicle Energy Expenditures

Dollars per Household 


\section{Row and Column Factors}

These tables present estimates of characteristics, vehicle fuel consumption, miles driven, and fuel efficiencies for all vehicles used for personal transportation in the United States. Since the estimates are based on a sample survey, they are subject to error. To help the reader compute an approximate relative standard error (RSE) for each of the estimates in the detailed tables, row and column factors are displayed on the top line and in the far-right column of each table. To calculate the RSE for a specific estimate, multiply the row factor by the column factor.

\section{Using Row and Column Factors to Estimate the Standard Error}

RSEs were calculated for all statistics in this publication, although they cannot be presented due to space limitations. However, the RSEs are presented in a generalized form. The method of presenting generalized RSEs of statistics uses sets of row and column factors inserted in the top row and right-most column of figures in each table. This method of presentation allows the readers to calculate an approximate RSE for each statistic. To estimate the RSE of a statistic

$$
R S E A_{i j}=R_{i} \times C_{j}
$$

in the $\mathrm{i}^{\text {th }}$ row and $\mathrm{j}^{\text {th }}$ column of a particular table, the approximation $R S E A_{i, j}$ for the original $R S E_{i, j}$ is given by: Where: $\mathrm{R}_{i}$ is the RSE row factor given at the right-most margin of row $\mathrm{i}$ in the tables, and $\mathrm{C}_{j}$ is the RSE column factor given at the top of column $\mathrm{j}$.

Total Vehicle-Miles Traveled in the Northeast Census Region $=299$ billion miles

$R$ (Northeast Census Region) $=5.7$

$\mathrm{C}($ Vehicle-Miles Traveled) $=1.1$

Approximate RSE

(Total Vehicle-Miles Traveled in the Northeast Census Region) $=(5.7) \times(1.1)=6.27$ percent

Approximate Standard Error

(Total Vehicle-Miles Traveled in the Northeast Census Region) $=(5.7) \times(1.1) \times 299 / 100$

$=18.75$ billion miles

Approximate 2 Standard Errors

$(95$ percent confidence interval $)=(1.96) \times(18.75)=36.74$ billion miles

Therefore, with approximately 95 percent confidence, the total vehicle-miles traveled in the Northeast Census

Region in 1994 was between 262.26 billion and 335.74 billion miles $(299 \pm 36.74)$

The following example illustrates this procedure:

Referencing the second row of Table 5.1 labeled "Northeast," and the third column labeled "Vehicle-Miles Traveled (billion)," yields an estimate of 299 billion miles driven. The RSE row factor is $R_{2}=5.7$, and the RSEcolumn factor is $C_{3}=1.1$. The approximate RSE for the estimate is, therefore,

$$
R S E A_{2,3}=5.7 \times 1.1=6.27 \text { percent }
$$

The standard error derived from row and column factors can be used to construct confidence intervals and to perform hypothesis tests by standard statistical methods. However, because the generalized variance procedure gives only 
approximate RSEs, such confidence intervals and statistical tests must also be regarded as only approximate. For the example above, the RSE determined directly by the half-sample method is actually 3.9 percent, not 6.27 percent.

\section{Determination of the Standard Error of the Difference Between Two Statistics}

The procedure used to compute the standard error of the difference between two statistics follows:

$$
S E\left(x_{i}-x_{2}\right)=\sqrt{\left[S E\left(x_{i}\right)\right]^{2}+\left[S E\left(x_{2}\right)\right]^{2}} .
$$

This procedure assumes the two statistics are not correlated. The following example illustrates this procedure. Households with children drove an average of 24,800 miles per household in 1994. Households without children drove an average of 18,900 miles, for a difference of 5,900 miles. The RSEs for households with and without children are 2.7 and 4.6, respectively. The corresponding standard errors are 670 miles and 869 miles, respectively. Therefore, the standard error for the difference is:

$$
\operatorname{SE}(5,900)=\sqrt{[670]^{2}+[869]^{2}}=1,097 \text { miles. }
$$

If 1.96 times the standard error is greater than the difference between the statistics, the difference is not statistically significant at the .05 level of significance (the level used to test significance of inferences in this report). In this example, 1.96 times the standard error equals 1,494 miles, while the difference is 6,300 miles. Therefore, the conclusion is that, in 1994, there was a significant difference in average mileage driven per household between households

with and without children. 

Table 5.1. U.S. Number of Vehicles, Vehicle-Miles, Motor Fuel Consumption and Expenditures, 1994

\begin{tabular}{|c|c|c|c|c|c|c|c|c|c|c|}
\hline \multirow{3}{*}{$\begin{array}{c}1993 \text { Household and } \\
1994 \text { Vehicle } \\
\text { Characteristics }\end{array}$} & \multicolumn{2}{|c|}{$\begin{array}{l}\text { Number of } \\
\text { Vehicles }\end{array}$} & \multicolumn{2}{|c|}{$\begin{array}{l}\text { Vehicie-Miles } \\
\text { Traveled }\end{array}$} & \multicolumn{3}{|c|}{$\begin{array}{l}\text { Motor Fuel } \\
\text { Consumption }\end{array}$} & \multicolumn{2}{|c|}{$\begin{array}{l}\text { Motor Fuel } \\
\text { Expenditures }\end{array}$} & \multirow{3}{*}{$\begin{array}{l}\text { RSE } \\
\text { Row } \\
\text { Factor: }\end{array}$} \\
\hline & (million) & (percent) & (billion) & (percent) & $\begin{array}{l}\text { (bilition } \\
\text { gallons) }\end{array}$ & $\begin{array}{l}\text { (gallon } \\
\text { percent) }\end{array}$ & $\begin{array}{l}\text { (quadrif- } \\
\text { lion Btu) }\end{array}$ & $\begin{array}{l}\text { (billion } \\
\text { dollars) }\end{array}$ & (percent) & \\
\hline & 0.9 & 0.8 & 1.1 & 1.0 & 1.1 & 1.0 & 1.1 & 1.1 & 1.0 & \\
\hline \multicolumn{11}{|l|}{ Household Characteristics } \\
\hline 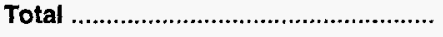 & 156.8 & 100.0 & 1,793 & 100.0 & 90.6 & 100.0 & 11.2 & 104.7 & 100.0 & 2.8 \\
\hline \multicolumn{11}{|l|}{ Census Region and Division } \\
\hline 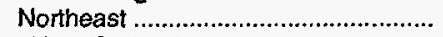 & 26.6 & 17.0 & 299 & 16.7 & 14.5 & 16.0 & 1.8 & 17.2 & 16.4 & 5.7 \\
\hline 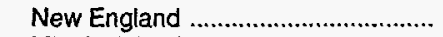 & 7.6 & 4.8 & 84 & 4.7 & 4.1 & 4.5 & 0.5 & 4.8 & 4.6 & 13.8 \\
\hline Middle Atlantic .................................... & 19.0 & 12.1 & 215 & 12.0 & 10.4 & 11.4 & 1.3 & 12.4 & 11.8 & 5.8 \\
\hline 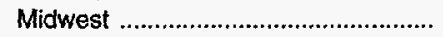 & 41.1 & 26.2 & 479 & 26.7 & 23.8 & 26.3 & 2.9 & 26.5 & 25.3 & 4.5 \\
\hline 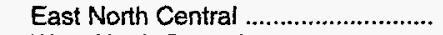 & 29.0 & 18.5 & 335 & 18.7 & 16.7 & 18.4 & 2.1 & 18.5 & 17.7 & 5.6 \\
\hline 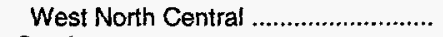 & 12.1 & 7.7 & 144 & 8.0 & 7.2 & 7.9 & 0.8 & 8.0 & 7.6 & 8.3 \\
\hline 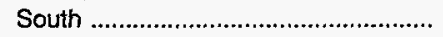 & 56.0 & 35.7 & 655 & 36.6 & 33.5 & 36.9 & 4.2 & 37.7 & 36.0 & 5.0 \\
\hline 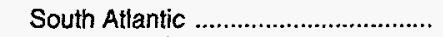 & 28.4 & 18.1 & 345 & 19.2 & 17.1 & 18.8 & 2.1 & 19.2 & 18.3 & 5.7 \\
\hline East South Central ............................. & 11.1 & 7.1 & 121 & 6.7 & 6.3 & 7.0 & 0.8 & 7.2 & 6.9 & 6.0 \\
\hline West South Central ............................ & 16.4 & 10.5 & 190 & 10.6 & 10.1 & 11.1 & 1.2 & 11.3 & 10.8 & 14.8 \\
\hline 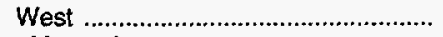 & 33.1 & 21.1 & 360 & 20.1 & 18.8 & 20.7 & 2.4 & 23.4 & 22.3 & 6.7 \\
\hline 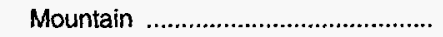 & 9.8 & 6.2 & 105 & 5.8 & 5.9 & 6.5 & 0.7 & 7.2 & 6.9 & 12.0 \\
\hline 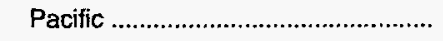 & 23.3 & 14.9 & 255 & 14.2 & 12.9 & 14.3 & 1.6 & 16.2 & 15.4 & 8.2 \\
\hline \multicolumn{11}{|l|}{ Largest Populated States } \\
\hline 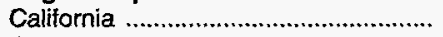 & 17.0 & 10.8 & 188 & 10.5 & 9.5 & 10.5 & 1.2 & +1.9 & 11.4 & 10.2 \\
\hline Florida & 8.8 & 5.6 & 104 & 5.8 & 5.1 & 5.6 & 0.6 & 5.8 & 5.6 & 5.3 \\
\hline 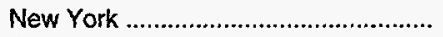 & 7.8 & 5.0 & 89 & 5.0 & 4.3 & 4.7 & 0.5 & 5.0 & 4.8 & 9.2 \\
\hline Texas & 11.0 & 7.0 & 124 & 6.9 & 6.6 & 7.3 & 0.8 & 7.3 & 7.0 & 16.6 \\
\hline \multicolumn{11}{|l|}{ Urban Status } \\
\hline Urban & 119.4 & 76.2 & 1,360 & 75.9 & 67.5 & 74.5 & 8.4 & 78.6 & 75.0 & 2.7 \\
\hline 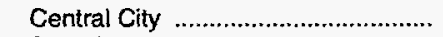 & 40.1 & 25.6 & 434 & 24.2 & 21.4 & 23.6 & 2.7 & 25.1 & 24.0 & 8.8 \\
\hline 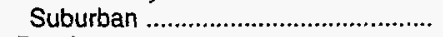 & 79.3 & 50.6 & 927 & 51.7 & 46.1 & 50.9 & 5.8 & 53.4 & 51.0 & 4.5 \\
\hline 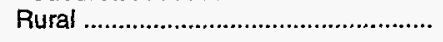 & 37.4 & 23.8 & 433 & 24.1 & 23.1 & 25.5 & 2.8 & 26.1 & 25.0 & 5.1 \\
\hline \multicolumn{11}{|l|}{ Household Size } \\
\hline 1 Person & 19.5 & 12.5 & 197 & 11.0 & 9.6 & 10.6 & 1.2 & 11.1 & 10.6 & 6.4 \\
\hline 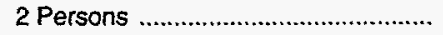 & 55.4 & 35.3 & 602 & 33.6 & 30.6 & 33.8 & 3.8 & 35.3 & 33.7 & 4.2 \\
\hline 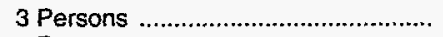 & 31.7 & 20.2 & 379 & 21.1 & 18.9 & 20.9 & 2.4 & 21.9 & 20.9 & 6.7 \\
\hline 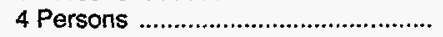 & 29.5 & 18.8 & 355 & 19.8 & 18.1 & 20.0 & 2.2 & 21.0 & 20.0 & 6.7 \\
\hline 5 Persons & 14.4 & 9.2 & 176 & 9.8 & 9.1 & 10.0 & 1.1 & 10.5 & 10.0 & 12.2 \\
\hline 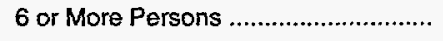 & 6.3 & 4.0 & 84 & 4.7 & 4.2 & 4.7 & 0.5 & 5.0 & 4.7 & 18.2 \\
\hline \multicolumn{11}{|l|}{ Household Composition } \\
\hline $\begin{array}{l}\text { Households with Children } \\
\text { Age of Oldest Child }\end{array}$ & 64.6 & 41.2 & 796 & 44.4 & 40.4 & 44.6 & 5.0 & 46.7 & 44.6 & 4.5 \\
\hline Under 7 Years ................................. & 17.1 & 10.9 & 206 & 11.5 & 10.3 & 11.4 & 1.3 & 12.0 & 11.4 & 10.1 \\
\hline 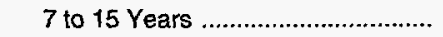 & 32.3 & 20.6 & 399 & 22.2 & 20.5 & 22.6 & 2.5 & 23.7 & 22.6 & 6.3 \\
\hline 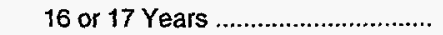 & 15.2 & 9.7 & 191 & 10.7 & 9.6 & 10.6 & 1.2 & 11.1 & 10.6 & 10.8 \\
\hline Households Without Children ............. & 92.2 & 58.8 & 997 & 55.6 & 50.2 & 55.4 & 6.2 & 58.0 & 55.4 & 3.4 \\
\hline $\begin{array}{l}\text { One Aduit .............................................. } \\
\text { Age of Householder }\end{array}$ & 19.5 & 12.5 & 197 & 11.0 & 9.6 & 10.6 & 1.2 & 11.1 & 10.6 & 6.4 \\
\hline Under 35 Years ............................ & 4.1 & 2.6 & 53 & 2.9 & 2.4 & 2.7 & 0.3 & 2.9 & 2.7 & 15.7 \\
\hline 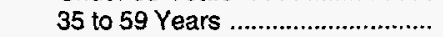 & 7.6 & 4.8 & 85 & 4.7 & 4.1 & 4.6 & 0.5 & 4.8 & 4.6 & 11.1 \\
\hline 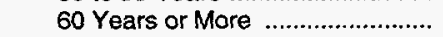 & 7.9 & 5.0 & 60 & 3.3 & 3.0 & 3.4 & 0.4 & 3.5 & 3.3 & 9.4 \\
\hline $\begin{array}{l}\text { Two or More Adults ........................... } \\
\text { Age of Householder }\end{array}$ & 72.6 & 46.3 & 800 & 44.6 & 40.6 & 44.8 & 5.0 & 46.9 & 44.8 & 3.9 \\
\hline Under 35 Years ............................ & 13.8 & 8.8 & 174 & 9.7 & 8.1 & 8.9 & 1.0 & 9.3 & 8.9 & 12.6 \\
\hline 35 to 59 Years ................................ & 33.9 & 21.6 & 393 & 21.9 & 20.2 & 22.3 & 2.5 & 23.3 & 22.3 & 6.9 \\
\hline 60 Years or More .......................... & 24.9 & 15.9 & 232 & 13.0 & 12.4 & 13.6 & 1.5 & 14.2 & 13.6 & 7.3 \\
\hline
\end{tabular}

See footnotes at end of table. 
Table 5.1. U.S. Number of Vehicles, Vehicle-Miles, Motor Fuel Consumption and Expenditures, 1994 (Continued)

\begin{tabular}{|c|c|c|c|c|c|c|c|c|c|c|}
\hline \multirow{3}{*}{$\begin{array}{l}1993 \text { Household and } \\
1994 \text { Vehicle } \\
\text { Characteristics }\end{array}$} & \multicolumn{2}{|c|}{$\begin{array}{l}\text { Number of } \\
\text { Vehicles }\end{array}$} & \multicolumn{2}{|c|}{$\begin{array}{l}\text { Vehicle-Miles } \\
\text { Traveled }\end{array}$} & \multicolumn{3}{|c|}{$\begin{array}{l}\text { Motor Fuel } \\
\text { Consumption }\end{array}$} & \multicolumn{2}{|c|}{$\begin{array}{l}\text { Motor Fuel } \\
\text { Expenditures }\end{array}$} & \multirow{3}{*}{$\begin{array}{l}\text { RSE } \\
\text { Row } \\
\text { Factor: }\end{array}$} \\
\hline & (million) & (percent) & (billion) & (percent) & $\begin{array}{l}\text { (billion } \\
\text { gallons) }\end{array}$ & $\begin{array}{c}\text { (gallon } \\
\text { percent) }\end{array}$ & $\begin{array}{l}\text { (quadril- } \\
\text { lion Btu) }\end{array}$ & $\begin{array}{l}\text { (billion } \\
\text { dollars) }\end{array}$ & (percent) & \\
\hline & 0.9 & 0.8 & 1.1 & 1.0 & 1.1 & 1.0 & 1.1 & 1.1 & 1.0 & \\
\hline \multicolumn{11}{|l|}{ Race of Householder } \\
\hline 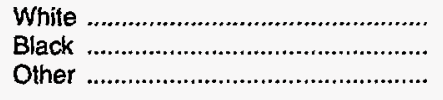 & $\begin{array}{r}138.6 \\
11.2 \\
7.0\end{array}$ & $\begin{array}{r}88.4 \\
7.1 \\
4.5\end{array}$ & $\begin{array}{r}1,592 \\
125 \\
77\end{array}$ & $\begin{array}{r}88.8 \\
7.0 \\
4.3\end{array}$ & $\begin{array}{r}80.5 \\
6.3 \\
3.7\end{array}$ & $\begin{array}{r}88.9 \\
7.0 \\
4.1\end{array}$ & $\begin{array}{r}10.0 \\
0.8 \\
0.5\end{array}$ & $\begin{array}{r}92.9 \\
7.3 \\
4.5\end{array}$ & $\begin{array}{r}88.7 \\
7.0 \\
4.3\end{array}$ & $\begin{array}{r}2.4 \\
13.1 \\
20.1\end{array}$ \\
\hline \multicolumn{11}{|l|}{ Hispanic Descent } \\
\hline Yes No & $\begin{array}{r}10.7 \\
146.1\end{array}$ & $\begin{array}{r}6.8 \\
93.2\end{array}$ & $\begin{array}{r}122 \\
1,671\end{array}$ & $\begin{array}{r}6.8 \\
93.2\end{array}$ & $\begin{array}{r}6.2 \\
84.4\end{array}$ & $\begin{array}{r}6.8 \\
93.2\end{array}$ & $\begin{array}{r}0.8 \\
10.4\end{array}$ & $\begin{array}{r}7.3 \\
97.4\end{array}$ & $\begin{array}{r}7.0 \\
93.0\end{array}$ & $\begin{array}{r}14.4 \\
2.9\end{array}$ \\
\hline 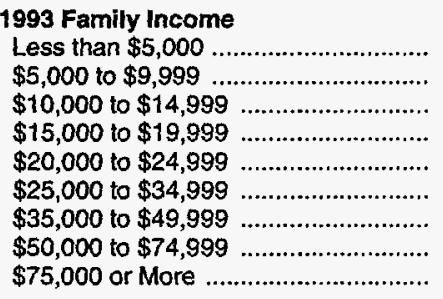 & $\begin{array}{r}3.1 \\
7.8 \\
12.5 \\
14.2 \\
14.4 \\
24.1 \\
33.6 \\
28.0 \\
19.1\end{array}$ & $\begin{array}{r}1.9 \\
5.0 \\
8.0 \\
9.1 \\
9.2 \\
15.4 \\
21.4 \\
17.9 \\
12.2\end{array}$ & $\begin{array}{r}35 \\
77 \\
128 \\
152 \\
158 \\
287 \\
390 \\
331 \\
234\end{array}$ & $\begin{array}{r}2.0 \\
4.3 \\
7.1 \\
8.5 \\
8.8 \\
16.0 \\
21.7 \\
18.5 \\
13.1\end{array}$ & $\begin{array}{r}1.7 \\
4.0 \\
6.6 \\
8.0 \\
8.0 \\
14.7 \\
19.5 \\
16.2 \\
11.9\end{array}$ & $\begin{array}{r}1.9 \\
4.4 \\
7.3 \\
8.8 \\
8.9 \\
16.2 \\
21.5 \\
17.9 \\
13.1\end{array}$ & $\begin{array}{l}0.2 \\
0.5 \\
0.8 \\
1.0 \\
1.0 \\
1.8 \\
2.4 \\
2.0 \\
1.5\end{array}$ & $\begin{array}{r}2.0 \\
4.6 \\
7.6 \\
9.1 \\
9.3 \\
16.9 \\
22.6 \\
18.7 \\
13.9\end{array}$ & $\begin{array}{r}1.9 \\
4.3 \\
7.3 \\
8.7 \\
8.9 \\
16.2 \\
21.5 \\
17.9 \\
13.3\end{array}$ & $\begin{array}{r}21.9 \\
14.8 \\
9.9 \\
10.1 \\
9.3 \\
6.4 \\
6.2 \\
7.0 \\
9.7\end{array}$ \\
\hline 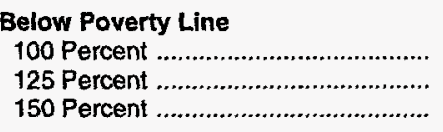 & $\begin{array}{l}12.4 \\
18.2 \\
25.7\end{array}$ & $\begin{array}{r}7.9 \\
11.6 \\
16.4\end{array}$ & $\begin{array}{l}133 \\
199 \\
285\end{array}$ & $\begin{array}{r}7.4 \\
11.1 \\
15.9\end{array}$ & $\begin{array}{r}6.9 \\
10.3 \\
14.7\end{array}$ & $\begin{array}{r}7.6 \\
11.4 \\
16.3\end{array}$ & $\begin{array}{l}0.9 \\
1.3 \\
1.8\end{array}$ & $\begin{array}{r}8.0 \\
11.9 \\
17.0\end{array}$ & $\begin{array}{r}7.7 \\
11.4 \\
16.3\end{array}$ & $\begin{array}{r}11.2 \\
10.4 \\
9.8\end{array}$ \\
\hline Eligible for Federal Assistance ${ }^{1} \ldots . .$. & 31.8 & 20.3 & 347 & 19.4 & 17.9 & 19.7 & 2.2 & 20.7 & 19.7 & 8.6 \\
\hline Number of Drivers ${ }^{2}$ (Fall 1993) & $\begin{array}{r}32.4 \\
91.6 \\
22.2 \\
9.7\end{array}$ & $\begin{array}{r}20.6 \\
58.5 \\
14.2 \\
6.2\end{array}$ & $\begin{array}{r}334 \\
1,061 \\
267 \\
124\end{array}$ & $\begin{array}{r}18.6 \\
59.2 \\
14.9 \\
6.9\end{array}$ & $\begin{array}{r}16.5 \\
54.3 \\
13.4 \\
6.0\end{array}$ & $\begin{array}{r}18.2 \\
59.9 \\
14.8 \\
6.6\end{array}$ & $\begin{array}{l}2.0 \\
6.7 \\
1.7 \\
0.7\end{array}$ & $\begin{array}{r}19.2 \\
62.8 \\
15.5 \\
6.9\end{array}$ & $\begin{array}{r}18.3 \\
59.9 \\
14.8 \\
6.6\end{array}$ & $\begin{array}{r}5.4 \\
3.3 \\
9.3 \\
14.0\end{array}$ \\
\hline 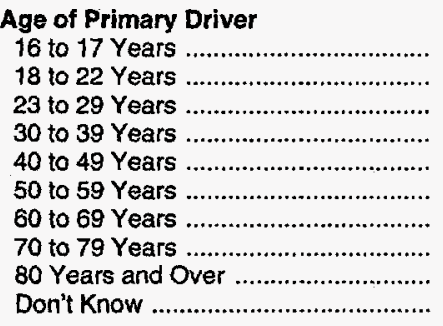 & $\begin{array}{r}1.1 \\
4.4 \\
8.4 \\
22.6 \\
22.3 \\
14.9 \\
10.6 \\
8.6 \\
2.6 \\
61.3\end{array}$ & $\begin{array}{r}0.7 \\
2.8 \\
5.3 \\
14.4 \\
14.2 \\
9.5 \\
6.8 \\
5.5 \\
1.6 \\
39.1\end{array}$ & $\begin{array}{r}11 \\
56 \\
111 \\
276 \\
276 \\
174 \\
99 \\
68 \\
16 \\
707\end{array}$ & $\begin{array}{r}0.6 \\
3.1 \\
6.2 \\
15.4 \\
15.4 \\
9.7 \\
5.5 \\
3.8 \\
0.9 \\
39.4\end{array}$ & $\begin{array}{r}0.5 \\
2.5 \\
5.1 \\
14.0 \\
14.0 \\
9.0 \\
5.3 \\
3.7 \\
0.9 \\
35.6\end{array}$ & $\begin{array}{r}0.6 \\
2.8 \\
5.6 \\
15.4 \\
15.5 \\
10.0 \\
5.8 \\
4.1 \\
1.0 \\
39.3\end{array}$ & $\begin{array}{l}0.1 \\
0.3 \\
0.6 \\
1.7 \\
1.7 \\
1.1 \\
0.7 \\
0.4 \\
0.1 \\
4.4\end{array}$ & $\begin{array}{r}0.6 \\
2.9 \\
5.9 \\
16.3 \\
16.3 \\
10.5 \\
6.2 \\
4.2 \\
1.0 \\
40.9\end{array}$ & $\begin{array}{r}0.5 \\
2.8 \\
5.6 \\
15.5 \\
15.6 \\
10.0 \\
5.9 \\
4.1 \\
1.0 \\
39.0\end{array}$ & $\begin{array}{r}32.4 \\
14.7 \\
9.8 \\
6.0 \\
5.9 \\
7.9 \\
9.5 \\
9.7 \\
18.5 \\
7.3\end{array}$ \\
\hline 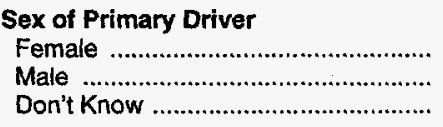 & $\begin{array}{l}43.4 \\
52.2 \\
61.2\end{array}$ & $\begin{array}{l}27.7 \\
33.3 \\
39.0\end{array}$ & $\begin{array}{l}506 \\
581 \\
706\end{array}$ & $\begin{array}{l}28.2 \\
32.4 \\
39.4\end{array}$ & $\begin{array}{l}23.5 \\
31.5 \\
35.6\end{array}$ & $\begin{array}{l}26.0 \\
34.7 \\
39.3\end{array}$ & $\begin{array}{l}2.9 \\
3.9 \\
4.4\end{array}$ & $\begin{array}{l}27.3 \\
36.5 \\
40.9\end{array}$ & $\begin{array}{l}26.1 \\
34.9 \\
39.0\end{array}$ & $\begin{array}{l}2.8 \\
3.1 \\
7.2\end{array}$ \\
\hline
\end{tabular}

See footnotes at end of table. 
Table 5.1. U.S. Number of Vehicles, Vehicle-Miles, Motor Fuel Consumption and Expenditures, 1994 (Continued)

\begin{tabular}{|c|c|c|c|c|c|c|c|c|c|c|}
\hline \multirow{3}{*}{$\begin{array}{c}1993 \text { Household and } \\
1994 \text { Vehicle } \\
\text { Characteristics }\end{array}$} & \multicolumn{2}{|c|}{$\begin{array}{l}\text { Number of } \\
\text { Vehicles }\end{array}$} & \multicolumn{2}{|c|}{$\begin{array}{l}\text { Vehicle-Miles } \\
\text { Traveled }\end{array}$} & \multicolumn{3}{|c|}{$\begin{array}{l}\text { Motor Fuel } \\
\text { Consumption }\end{array}$} & \multicolumn{2}{|c|}{$\begin{array}{l}\text { Motor Fuel } \\
\text { Expenditures }\end{array}$} & \multirow{3}{*}{$\begin{array}{l}\text { RSE } \\
\text { Row } \\
\text { Factor }\end{array}$} \\
\hline & (million) & (percent) & (billion) & (percent) & $\begin{array}{l}\text { (billion } \\
\text { gallons) }\end{array}$ & $\begin{array}{l}\text { (gallon } \\
\text { percent) }\end{array}$ & $\begin{array}{l}\text { (quadril- } \\
\text { lion Btu) }\end{array}$ & $\begin{array}{l}\text { (billion } \\
\text { dollars) }\end{array}$ & (percent) & \\
\hline & 0.9 & 0.8 & 1.1 & 1.0 & 1.1 & 1.0 & 1.1 & 1.1 & 1.0 & \\
\hline \multicolumn{11}{|l|}{$\begin{array}{l}\text { Average Number of Vehicles per } \\
\text { Household During the Year }\end{array}$} \\
\hline $\begin{array}{l}\text { Part-Year Vehicle } \\
\text { Only } 1 \\
\text { Between } 1 \text { and } 2 \\
\text { Only } 2 \\
\text { Between } 2 \text { and } 3 \\
\text { Only } 3 \\
\text { Between } 3 \text { and } 4 \\
4 \text { or More }\end{array}$ & $\begin{array}{r}Q \\
Q \\
28.6 \\
8.4 \\
56.9 \\
13.5 \\
25.2 \\
7.9 \\
14.8\end{array}$ & $\begin{array}{r}Q \\
18.2 \\
5.4 \\
36.3 \\
8.6 \\
16.1 \\
5.1 \\
9.5\end{array}$ & $\begin{array}{l}Q \\
306 \\
107 \\
659 \\
163 \\
274 \\
100 \\
169\end{array}$ & $\begin{array}{r}\mathrm{Q} \\
17.1 \\
6.0 \\
36.7 \\
9.1 \\
15.3 \\
5.6 \\
9.4\end{array}$ & $\begin{array}{r}\text { Q } \\
14.8 \\
5.4 \\
32.9 \\
8.5 \\
14.5 \\
4.9 \\
8.7\end{array}$ & $\begin{array}{r}Q \\
16.4 \\
6.0 \\
36.4 \\
9.4 \\
16.0 \\
5.5 \\
9.6\end{array}$ & $\begin{array}{l}Q \\
1.8 \\
0.7 \\
4.1 \\
1.1 \\
1.8 \\
0.6 \\
1.1\end{array}$ & \begin{tabular}{r}
\multicolumn{1}{c}{$Q$} \\
17.3 \\
6.3 \\
38.1 \\
9.8 \\
16.8 \\
5.6 \\
10.1
\end{tabular} & $\begin{array}{r}Q \\
16.5 \\
6.0 \\
36.4 \\
9.4 \\
16.0 \\
5.4 \\
9.6\end{array}$ & $\begin{array}{r}\text { NF } \\
6.0 \\
13.6 \\
4.5 \\
8.7 \\
7.9 \\
14.8 \\
12.5\end{array}$ \\
\hline \multicolumn{11}{|l|}{ Vehicle Characteristics } \\
\hline \multicolumn{11}{|l|}{ Model Year } \\
\hline 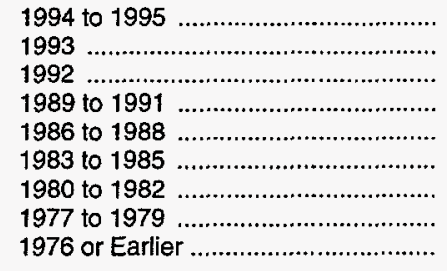 & $\begin{array}{r}7.2 \\
10.4 \\
11.4 \\
35.0 \\
36.3 \\
25.6 \\
10.9 \\
9.5 \\
10.6\end{array}$ & $\begin{array}{r}4.6 \\
6.6 \\
7.3 \\
22.3 \\
23.1 \\
16.3 \\
7.0 \\
6.0 \\
6.8\end{array}$ & $\begin{array}{r}103 \\
142 \\
149 \\
436 \\
411 \\
270 \\
107 \\
88 \\
87\end{array}$ & $\begin{array}{r}5.7 \\
7.9 \\
8.3 \\
24.3 \\
22.9 \\
15.1 \\
6.0 \\
4.9 \\
4.9\end{array}$ & $\begin{array}{r}5.0 \\
6.6 \\
6.9 \\
20.7 \\
19.0 \\
13.3 \\
5.6 \\
6.3 \\
7.2\end{array}$ & $\begin{array}{r}5.5 \\
7.3 \\
7.7 \\
22.8 \\
20.9 \\
14.7 \\
6.1 \\
7.0 \\
7.9\end{array}$ & $\begin{array}{l}0.6 \\
0.8 \\
0.9 \\
2.6 \\
2.4 \\
1.7 \\
0.7 \\
0.8 \\
0.9\end{array}$ & $\begin{array}{r}5.8 \\
7.7 \\
8.0 \\
23.9 \\
22.0 \\
15.3 \\
6.4 \\
7.3 \\
8.2\end{array}$ & $\begin{array}{r}5.5 \\
7.4 \\
7.7 \\
22.8 \\
21.0 \\
14.6 \\
6.1 \\
7.0 \\
7.8\end{array}$ & $\begin{array}{r}9.8 \\
7.3 \\
7.1 \\
4.1 \\
5.0 \\
6.5 \\
10.5 \\
12.9 \\
13.9\end{array}$ \\
\hline \multicolumn{11}{|l|}{ Type of Vehicle } \\
\hline 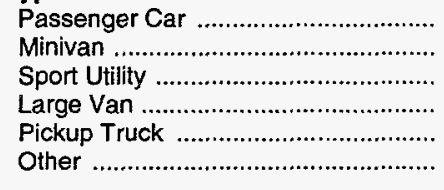 & $\begin{array}{r}106.4 \\
8.1 \\
9.5 \\
3.4 \\
28.8 \\
Q\end{array}$ & $\begin{array}{r}67.9 \\
5.2 \\
6.1 \\
2.2 \\
18.4 \\
Q\end{array}$ & $\begin{array}{r}1,200 \\
108 \\
121 \\
40 \\
320 \\
Q\end{array}$ & $\begin{array}{r}66.9 \\
6.0 \\
6.7 \\
2.2 \\
17.8 \\
Q\end{array}$ & $\begin{array}{r}54.7 \\
5.5 \\
7.4 \\
2.9 \\
19.6 \\
Q\end{array}$ & $\begin{array}{r}60.4 \\
6.1 \\
8.2 \\
3.2 \\
21.7 \\
Q\end{array}$ & $\begin{array}{l}6.8 \\
0.7 \\
0.9 \\
0.4 \\
2.4 \\
Q\end{array}$ & $\begin{array}{r}63.3 \\
6.3 \\
8.7 \\
3.3 \\
22.6 \\
Q\end{array}$ & $\begin{array}{c}60.4 \\
6.0 \\
8.3 \\
3.1 \\
21.6 \\
Q\end{array}$ & $\begin{array}{r}2.5 \\
8.5 \\
8.9 \\
14.9 \\
5.4 \\
\text { NF }\end{array}$ \\
\hline \multicolumn{11}{|l|}{ Fuel Efficiency (miles per gallon) } \\
\hline 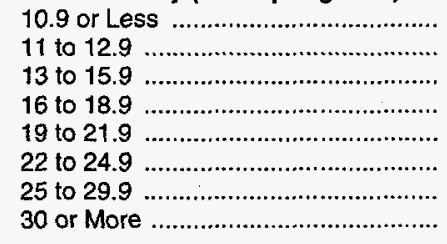 & $\begin{array}{r}7.0 \\
8.9 \\
18.9 \\
26.5 \\
35.2 \\
28.2 \\
24.9 \\
7.2\end{array}$ & $\begin{array}{r}4.5 \\
5.7 \\
12.0 \\
16.9 \\
22.4 \\
18.0 \\
15.9 \\
4.6\end{array}$ & $\begin{array}{r}39 \\
79 \\
183 \\
278 \\
423 \\
361 \\
324 \\
105\end{array}$ & $\begin{array}{r}2.2 \\
4.4 \\
10.2 \\
15.5 \\
23.6 \\
20.1 \\
18.1 \\
5.9\end{array}$ & $\begin{array}{r}4.1 \\
6.5 \\
12.6 \\
15.9 \\
20.8 \\
15.5 \\
12.0 \\
3.2\end{array}$ & $\begin{array}{r}4.6 \\
7.2 \\
13.9 \\
17.5 \\
22.9 \\
17.1 \\
13.3 \\
3.5\end{array}$ & $\begin{array}{l}0.5 \\
0.8 \\
1.6 \\
2.0 \\
2.6 \\
1.9 \\
1.5 \\
0.4\end{array}$ & $\begin{array}{r}4.7 \\
7.6 \\
14.5 \\
18.4 \\
24.2 \\
17.8 \\
13.8 \\
3.6\end{array}$ & $\begin{array}{r}4.5 \\
7.3 \\
13.9 \\
17.6 \\
23.1 \\
17.0 \\
13.2 \\
3.5\end{array}$ & $\begin{array}{r}13.0 \\
12.0 \\
7.2 \\
6.0 \\
5.1 \\
6.1 \\
5.2 \\
13.5\end{array}$ \\
\hline 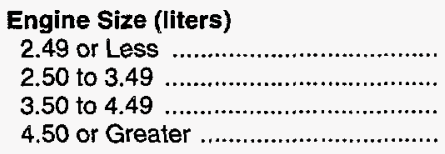 & $\begin{array}{l}59.8 \\
30.1 \\
24.0 \\
42.8\end{array}$ & $\begin{array}{l}38.2 \\
19.2 \\
15.3 \\
27.3\end{array}$ & $\begin{array}{l}699 \\
364 \\
283 \\
448\end{array}$ & $\begin{array}{l}39.0 \\
20.3 \\
15.8 \\
25.0\end{array}$ & $\begin{array}{l}27.4 \\
17.5 \\
15.1 \\
30.6\end{array}$ & $\begin{array}{l}30.3 \\
19.3 \\
16.6 \\
33.8\end{array}$ & $\begin{array}{l}3.4 \\
2.2 \\
1.9 \\
3.8\end{array}$ & $\begin{array}{l}31.7 \\
20.3 \\
17.3 \\
35.5\end{array}$ & $\begin{array}{l}30.2 \\
19.4 \\
16.5 \\
33.9\end{array}$ & $\begin{array}{l}3.9 \\
4.6 \\
6.5 \\
4.8\end{array}$ \\
\hline
\end{tabular}

See footnotes at end of table. 
Table 5.1. U.S. Number of Vehicles, Vehicle-Miles, Motor Fuel Consumption and Expenditures, 1994 (Continued)

\begin{tabular}{|c|c|c|c|c|c|c|c|c|c|c|}
\hline \multirow{3}{*}{$\begin{array}{l}1993 \text { Household and } \\
1994 \text { Vehicle } \\
\text { Characteristics }\end{array}$} & \multicolumn{2}{|c|}{$\begin{array}{c}\text { Number of } \\
\text { Vehicles }\end{array}$} & \multicolumn{2}{|c|}{$\begin{array}{c}\text { Vehicle-Miles } \\
\text { Traveled }\end{array}$} & \multicolumn{3}{|c|}{$\begin{array}{l}\text { Motor Fuel } \\
\text { Consumption }\end{array}$} & \multicolumn{2}{|c|}{$\begin{array}{l}\text { Motor Fuel } \\
\text { Expenditures }\end{array}$} & \multirow{3}{*}{$\begin{array}{l}\text { RSE } \\
\text { Row } \\
\text { Factor: }\end{array}$} \\
\hline & (million) & (percent) & (billion) & (percent) & $\begin{array}{l}\text { (billion } \\
\text { gallons) }\end{array}$ & $\begin{array}{l}\text { (gallon } \\
\text { percent) }\end{array}$ & $\begin{array}{l}\text { (quadril- } \\
\text { lion Btu) }\end{array}$ & $\begin{array}{l}\text { (billion } \\
\text { dollars) }\end{array}$ & (percent) & \\
\hline & 0.9 & 0.8 & 1.1 & 1.0 & 1.1 & 1.0 & 1.1 & 1.1 & 1.0 & \\
\hline Number of Cylinders & & & & & & & & & & \\
\hline Other 6 & $\begin{array}{r}61.5 \\
52.3 \\
41.6 \\
1.3\end{array}$ & $\begin{array}{r}39.2 \\
33.4 \\
26.6 \\
0.8\end{array}$ & $\begin{array}{r}713 \\
631 \\
435 \\
14\end{array}$ & $\begin{array}{r}39.8 \\
35.2 \\
24.3 \\
0.8\end{array}$ & $\begin{array}{r}28.3 \\
32.3 \\
29.4 \\
0.5\end{array}$ & $\begin{array}{r}31.2 \\
35.7 \\
32.5 \\
0.6\end{array}$ & $\begin{array}{l}3.5 \\
4.0 \\
3.6 \\
0.1\end{array}$ & $\begin{array}{r}32.6 \\
37.4 \\
34.1 \\
0.7\end{array}$ & $\begin{array}{r}31.2 \\
35.7 \\
32.5 \\
0.6\end{array}$ & $\begin{array}{r}3.5 \\
3.5 \\
4.8 \\
25.8\end{array}$ \\
\hline 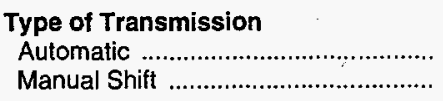 & $\begin{array}{r}117.5 \\
39.3\end{array}$ & $\begin{array}{l}75.0 \\
25.0\end{array}$ & $\begin{array}{r}1,337 \\
456\end{array}$ & $\begin{array}{l}74.6 \\
25.4\end{array}$ & $\begin{array}{l}69.8 \\
20.7\end{array}$ & $\begin{array}{l}77.1 \\
22.9\end{array}$ & $\begin{array}{l}8.6 \\
2.6\end{array}$ & $\begin{array}{l}80.6 \\
24.1\end{array}$ & $\begin{array}{l}77.0 \\
23.0\end{array}$ & $\begin{array}{l}2.2 \\
5.6\end{array}$ \\
\hline 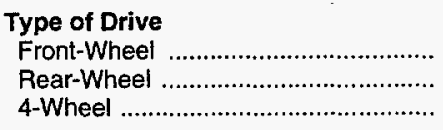 & $\begin{array}{l}74.9 \\
64.3 \\
17.6\end{array}$ & $\begin{array}{l}47.8 \\
41.0 \\
11.2\end{array}$ & $\begin{array}{l}891 \\
693 \\
209\end{array}$ & $\begin{array}{l}49.7 \\
38.6 \\
11.7\end{array}$ & $\begin{array}{l}38.1 \\
39.7 \\
12.7\end{array}$ & $\begin{array}{l}42.1 \\
43.9 \\
14.0\end{array}$ & $\begin{array}{l}4.7 \\
4.9 \\
1.6\end{array}$ & $\begin{array}{l}44.0 \\
45.8 \\
14.9\end{array}$ & $\begin{array}{l}42.0 \\
43.7 \\
14.2\end{array}$ & $\begin{array}{l}3.0 \\
4.0 \\
7.4\end{array}$ \\
\hline 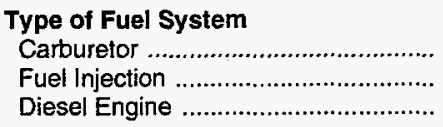 & $\begin{array}{r}76.4 \\
78.2 \\
2.2\end{array}$ & $\begin{array}{r}48.7 \\
49.9 \\
1.4\end{array}$ & $\begin{array}{r}802 \\
966 \\
25\end{array}$ & $\begin{array}{r}44.7 \\
53.9 \\
1.4\end{array}$ & $\begin{array}{r}43.9 \\
45.3 \\
1.4\end{array}$ & $\begin{array}{r}48.4 \\
50.0 \\
1.6\end{array}$ & $\begin{array}{l}5.4 \\
5.6 \\
0.2\end{array}$ & $\begin{array}{r}50.6 \\
52.4 \\
1.8\end{array}$ & $\begin{array}{r}48.3 \\
50.0 \\
1.7\end{array}$ & $\begin{array}{r}3.9 \\
2.9 \\
19.7\end{array}$ \\
\hline 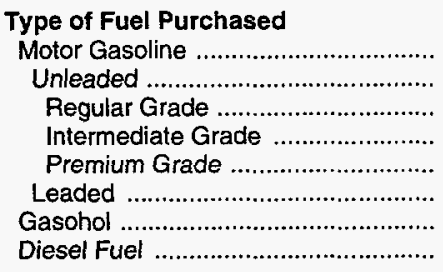 & $\begin{array}{c}153.4 \\
151.5 \\
104.2 \\
20.6 \\
26.7 \\
Q \\
1.4 \\
1.8\end{array}$ & $\begin{array}{c}97.9 \\
96.7 \\
66.4 \\
13.2 \\
17.1 \\
Q \\
0.9 \\
1.1\end{array}$ & $\begin{array}{c}1,752 \\
1,736 \\
1,199 \\
238 \\
299 \\
Q \\
17 \\
22\end{array}$ & $\begin{array}{c}97.7 \\
96.8 \\
66.9 \\
13.3 \\
16.6 \\
Q \\
0.9 \\
1.2\end{array}$ & $\begin{array}{c}88.3 \\
87.0 \\
59.9 \\
11.7 \\
15.4 \\
Q \\
0.9 \\
1.2\end{array}$ & $\begin{array}{c}97.5 \\
96.1 \\
66.1 \\
12.9 \\
17.0 \\
Q \\
1.0 \\
1.3\end{array}$ & $\begin{array}{c}11.0 \\
10.9 \\
7.5 \\
1.5 \\
1.9 \\
Q \\
0.0 \\
0.2\end{array}$ & $\begin{array}{c}102.0 \\
100.6 \\
66.3 \\
14.2 \\
20.0 \\
Q \\
1.0 \\
1.5\end{array}$ & $\begin{array}{c}97.4 \\
96.0 \\
63.3 \\
13.6 \\
19.1 \\
Q \\
1.0 \\
1.4\end{array}$ & $\begin{array}{c}2.8 \\
2.8 \\
2.5 \\
6.5 \\
6.4 \\
\text { NF } \\
26.6 \\
22.1\end{array}$ \\
\hline 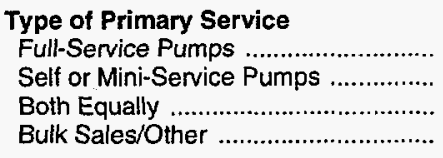 & $\begin{array}{r}13.8 \\
139.8 \\
2.9 \\
Q\end{array}$ & $\begin{array}{r}8.8 \\
89.2 \\
1.8 \\
Q\end{array}$ & $\begin{array}{r}137 \\
1,624 \\
30 \\
Q\end{array}$ & $\begin{array}{c}7.6 \\
90.5 \\
1.6 \\
Q\end{array}$ & $\begin{array}{r}6.8 \\
82.0 \\
1.6 \\
Q\end{array}$ & $\begin{array}{r}7.5 \\
90.5 \\
1.7 \\
Q\end{array}$ & $\begin{array}{c}0.8 \\
10.2 \\
0.2 \\
Q\end{array}$ & $\begin{array}{r}8.0 \\
94.6 \\
1.9 \\
Q\end{array}$ & $\begin{array}{l}7.7 \\
90.3 \\
1.8 \\
Q\end{array}$ & $\begin{array}{r}16.1 \\
2.2 \\
28.2 \\
\text { NF }\end{array}$ \\
\hline $\begin{array}{l}\text { Vehicle Used for Commuting } \\
\text { to and from Work } \\
\text { Yes }\end{array}$ & $\begin{array}{l}95.3 \\
61.5\end{array}$ & $\begin{array}{l}60.8 \\
39.2\end{array}$ & $\begin{array}{r}1,175 \\
618\end{array}$ & $\begin{array}{l}65.5 \\
34.5\end{array}$ & $\begin{array}{l}58.2 \\
32.4\end{array}$ & $\begin{array}{l}64.2 \\
35.8\end{array}$ & $\begin{array}{l}7.2 \\
4.0\end{array}$ & $\begin{array}{l}67.3 \\
37.5\end{array}$ & $\begin{array}{l}64.2 \\
35.8\end{array}$ & $\begin{array}{l}2.6 \\
4.1\end{array}$ \\
\hline
\end{tabular}

1 Below 150 percent of poverty line or 60 percent of median State income.

2 Approximately 0.5 percent of the vehicle stock was owned by households that had no drivers as of fall 1993.

$\mathrm{NF}=$ No applicable RSE row factor.

$Q=$ Data withheld either because the Relative Standard Error (RSE) was greater than 50 percent or fewer than 10 households were sampled.

Notes: - "Households with Children" category includes members under age 18 years old unless the member is the householder or spouse. - To obtain the Relative Standard Error (RSE) percentage for any table cell, multiply the corresponding column and row factors. • Because of rounding, data may not sum to totals. - Data in this table are for households with vehicles for personal transportation. - See Glossary for definition of terms used in this report.

Source: Energy Information Administration, Office of Energy Markets and End Use, Form ElA-457 A of the 1993 Residential Energy Consumption Survey and Forms EIA-876 A, B, C, and D of the 1994 Residential Transportation Energy Consumption Survey. 
Table 5.2. U.S. per Household Vehicle-Miles Traveled, Vehicle Fuel Consumption and Expenditures, 1994

\begin{tabular}{|c|c|c|c|c|c|c|}
\hline \multirow[b]{2}{*}{$\begin{array}{l}1993 \text { Household } \\
\text { Characteristics }\end{array}$} & \multirow[b]{2}{*}{$\begin{array}{l}\text { Number of } \\
\text { Households } \\
\text { (million) }\end{array}$} & \multicolumn{4}{|c|}{ Average per Household } & \multirow[b]{2}{*}{$\begin{array}{l}\text { RSE } \\
\text { Row } \\
\text { Factor: }\end{array}$} \\
\hline & & $\begin{array}{l}\text { Number of } \\
\text { Vehicles }\end{array}$ & $\begin{array}{c}\text { Vehicle-Miles } \\
\text { Traveled } \\
\text { (thousands) }\end{array}$ & $\begin{array}{l}\text { Consumption } \\
\text { (gallons) }\end{array}$ & $\begin{array}{l}\text { Expenditures } \\
\text { (dollars) }\end{array}$ & \\
\hline \multicolumn{7}{|l|}{ Household Characteristics } \\
\hline 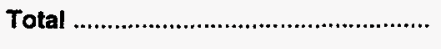 & 84.9 & 1.8 & 21.1 & 1,067 & 1,234 & 1.8 \\
\hline \multicolumn{7}{|l|}{ Census Region and Division } \\
\hline 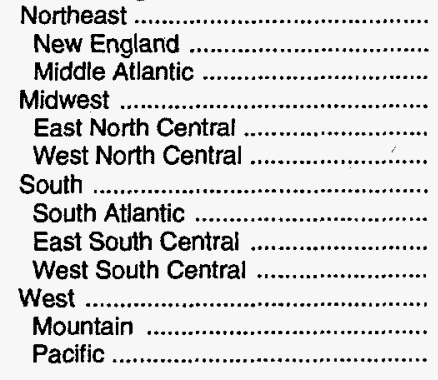 & $\begin{array}{r}14.7 \\
4.1 \\
10.7 \\
21.6 \\
15.1 \\
6.5 \\
30.2 \\
15.4 \\
5.4 \\
9.4 \\
18.4 \\
5.4 \\
13.0\end{array}$ & $\begin{array}{l}1.8 \\
1.9 \\
1.8 \\
1.9 \\
1.9 \\
1.9 \\
1.9 \\
1.8 \\
2.0 \\
1.8 \\
1.8 \\
1.8 \\
1.8\end{array}$ & $\begin{array}{l}20.3 \\
20.5 \\
20.2 \\
22.2 \\
22.2 \\
22.2 \\
21.7 \\
22.4 \\
22.2 \\
20.3 \\
19.6 \\
19.6 \\
19.6\end{array}$ & $\begin{array}{r}982 \\
1,006 \\
973 \\
1,104 \\
1,102 \\
1,110 \\
1,109 \\
1,111 \\
1,167 \\
1,073 \\
1,023 \\
1,093 \\
994\end{array}$ & $\begin{array}{l}1,166 \\
1,180 \\
1,161 \\
1,226 \\
1,225 \\
1,229 \\
1,249 \\
1,250 \\
1,323 \\
1,204 \\
1,271 \\
1,344 \\
1,241\end{array}$ & $\begin{array}{l}3.8 \\
5.7 \\
4.3 \\
3.5 \\
4.6 \\
5.1 \\
2.8 \\
3.9 \\
5.4 \\
5.4 \\
4.6 \\
8.5 \\
5.2\end{array}$ \\
\hline 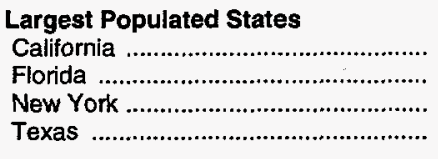 & $\begin{array}{l}9.5 \\
4.9 \\
4.7 \\
6.1\end{array}$ & $\begin{array}{l}1.8 \\
1.8 \\
1.6 \\
1.8\end{array}$ & $\begin{array}{l}19.8 \\
20.9 \\
18.7 \\
20.3\end{array}$ & $\begin{array}{r}1,000 \\
1,030 \\
898 \\
1,076\end{array}$ & $\begin{array}{l}1,254 \\
1,175 \\
1,062 \\
1,203\end{array}$ & $\begin{array}{l}6.1 \\
6.6 \\
7.5 \\
4.9\end{array}$ \\
\hline 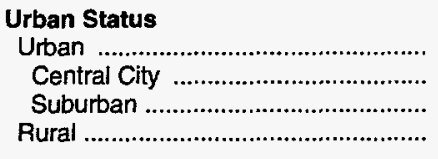 & $\begin{array}{l}65.6 \\
24.1 \\
41.5 \\
19.3\end{array}$ & $\begin{array}{l}1.8 \\
1.7 \\
1.9 \\
1.9\end{array}$ & $\begin{array}{l}20.7 \\
18.0 \\
22.3 \\
22.5\end{array}$ & $\begin{array}{r}1,029 \\
888 \\
1,111 \\
1,196\end{array}$ & $\begin{array}{l}1,198 \\
1,042 \\
1,289 \\
1,355\end{array}$ & $\begin{array}{l}2.2 \\
4.2 \\
2.6 \\
3.2\end{array}$ \\
\hline 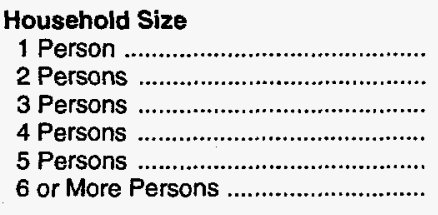 & $\begin{array}{r}17.0 \\
30.1 \\
15.0 \\
13.3 \\
6.7 \\
2.7\end{array}$ & $\begin{array}{l}1.2 \\
1.8 \\
2.1 \\
2.2 \\
2.2 \\
2.3\end{array}$ & $\begin{array}{l}11.6 \\
20.0 \\
25.2 \\
26.6 \\
26.3 \\
30.9\end{array}$ & $\begin{array}{r}566 \\
1,016 \\
1,257 \\
1,357 \\
1,359 \\
1,566\end{array}$ & $\begin{array}{r}657 \\
1,171 \\
1,455 \\
1,570 \\
1,571 \\
1,829\end{array}$ & $\begin{array}{r}4.6 \\
2.6 \\
3.5 \\
4.0 \\
5.6 \\
11.9\end{array}$ \\
\hline \multicolumn{7}{|l|}{ Household Composition } \\
\hline 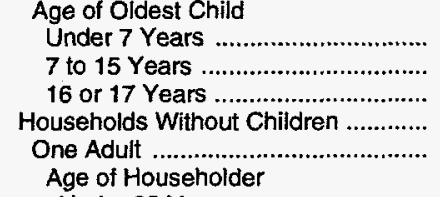 & $\begin{array}{r}9.2 \\
16.5 \\
6.4 \\
52.7 \\
17.0\end{array}$ & $\begin{array}{l}1.8 \\
2.0 \\
2.4 \\
1.7 \\
1.2\end{array}$ & $\begin{array}{l}22.4 \\
24.2 \\
29.9 \\
18.9 \\
11.6\end{array}$ & $\begin{array}{r}1,119 \\
1,240 \\
1,500 \\
951 \\
566\end{array}$ & $\begin{array}{r}1,296 \\
1,434 \\
1,727 \\
1,100 \\
657\end{array}$ & $\begin{array}{l}5.7 \\
3.3 \\
5.3 \\
2.3 \\
4.6\end{array}$ \\
\hline 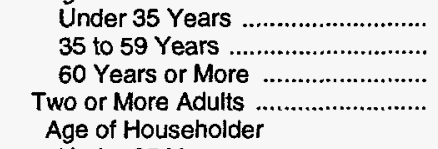 & $\begin{array}{r}3.4 \\
6.6 \\
7.0 \\
35.8\end{array}$ & $\begin{array}{l}1.2 \\
1.1 \\
1.1 \\
2.0\end{array}$ & $\begin{array}{r}15.6 \\
12.8 \\
8.6 \\
22.4\end{array}$ & $\begin{array}{r}720 \\
627 \\
435 \\
1,134\end{array}$ & $\begin{array}{r}843 \\
728 \\
498 \\
1,311\end{array}$ & $\begin{array}{r}12.4 \\
6.5 \\
6.0 \\
2.6\end{array}$ \\
\hline 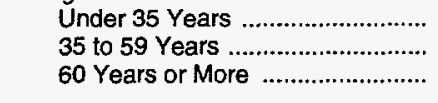 & $\begin{array}{r}7.2 \\
15.0 \\
13.6\end{array}$ & $\begin{array}{l}1.9 \\
2.3 \\
1.8\end{array}$ & $\begin{array}{l}24.4 \\
26.2 \\
17.1\end{array}$ & $\begin{array}{r}1,126 \\
1,344 \\
907\end{array}$ & $\begin{array}{l}1,304 \\
1,557 \\
1,044\end{array}$ & $\begin{array}{l}6.4 \\
4.0 \\
4.6\end{array}$ \\
\hline \multicolumn{7}{|l|}{ Race of Householder } \\
\hline 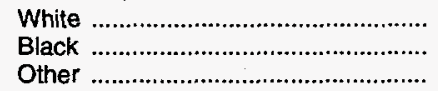 & $\begin{array}{r}73.3 \\
7.3 \\
4.3\end{array}$ & $\begin{array}{l}1.9 \\
1.5 \\
1.6\end{array}$ & $\begin{array}{l}21.7 \\
17.1 \\
17.8\end{array}$ & $\begin{array}{r}1,099 \\
865 \\
867\end{array}$ & $\begin{array}{l}1,267 \\
1,005 \\
1,053\end{array}$ & $\begin{array}{r}1.8 \\
7.4 \\
10.7\end{array}$ \\
\hline
\end{tabular}

See footnotes at end of table. 
Table 5.2. U.S. per Household Vehicle-Miles Traveled, Vehicle Fuel Consumption and Expenditures, 1994 (Continued)

\begin{tabular}{|c|c|c|c|c|c|c|}
\hline \multirow[b]{2}{*}{$\begin{array}{l}1993 \text { Household } \\
\text { Characteristics }\end{array}$} & \multirow[b]{2}{*}{$\begin{array}{c}\text { Number of } \\
\text { Households } \\
\text { (million) }\end{array}$} & \multicolumn{4}{|c|}{ Average per Household } & \multirow{3}{*}{$\begin{array}{l}\text { RSE } \\
\text { Row } \\
\text { Factor: }\end{array}$} \\
\hline & & $\begin{array}{l}\text { Number of } \\
\text { Vehicles }\end{array}$ & $\begin{array}{l}\text { Vehicle-Miles } \\
\text { Traveled } \\
\text { (thousands) }\end{array}$ & $\begin{array}{l}\text { Consumption } \\
\text { (gallons) }\end{array}$ & $\begin{array}{l}\text { Expenditures } \\
\text { (dollars) }\end{array}$ & \\
\hline RSE Column Factor: & 1.5 & 0.6 & 1.0 & 1.0 & 1.0 & \\
\hline Hispanic Descent & & & & & & \\
\hline Yes & $\begin{array}{r}6.3 \\
78.6\end{array}$ & $\begin{array}{l}1.7 \\
1.9\end{array}$ & $\begin{array}{l}19.5 \\
21.3\end{array}$ & $\begin{array}{r}990 \\
1,073\end{array}$ & $\begin{array}{l}1,174 \\
1,238\end{array}$ & $\begin{array}{l}8.2 \\
1.9\end{array}$ \\
\hline 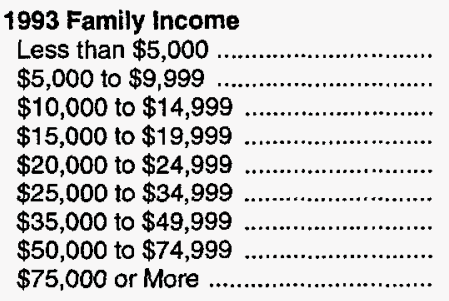 & $\begin{array}{r}2.2 \\
6.3 \\
8.9 \\
8.5 \\
8.6 \\
13.3 \\
16.5 \\
12.3 \\
8.2\end{array}$ & $\begin{array}{l}1.4 \\
1.2 \\
1.4 \\
1.7 \\
1.7 \\
1.8 \\
2.0 \\
2.3 \\
2.3\end{array}$ & $\begin{array}{l}16.1 \\
12.2 \\
14.3 \\
17.8 \\
18.4 \\
21.6 \\
23.6 \\
27.0 \\
28.5\end{array}$ & $\begin{array}{r}781 \\
631 \\
739 \\
937 \\
931 \\
1,102 \\
1,182 \\
1,325 \\
1,443\end{array}$ & $\begin{array}{r}923 \\
719 \\
854 \\
1,073 \\
1,079 \\
1,269 \\
1,366 \\
1,528 \\
1,692\end{array}$ & $\begin{array}{r}17.4 \\
9.6 \\
6.0 \\
6.9 \\
6.6 \\
4.2 \\
3.7 \\
4.7 \\
5.2\end{array}$ \\
\hline 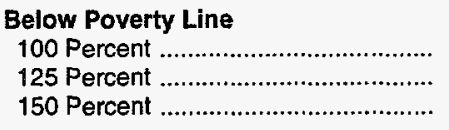 & $\begin{array}{r}9.0 \\
13.0 \\
17.4\end{array}$ & $\begin{array}{l}1.4 \\
1.4 \\
1.5\end{array}$ & $\begin{array}{l}14.7 \\
15.3 \\
16.4\end{array}$ & $\begin{array}{l}769 \\
795 \\
846\end{array}$ & $\begin{array}{l}890 \\
918 \\
979\end{array}$ & $\begin{array}{l}7.3 \\
6.7 \\
6.0\end{array}$ \\
\hline Eligible for Federal Assistance ${ }^{1} \ldots . .$. & 21.6 & 1.5 & 16.1 & 828 & 957 & 5.1 \\
\hline $\begin{array}{l}\text { Number of Drivers } \\
1 \\
2 \\
3 \\
4 \text { or More (Fall 1993) }\end{array}$ & $\begin{array}{r}27.3 \\
45.7 \\
8.0 \\
2.9\end{array}$ & $\begin{array}{l}1.2 \\
2.0 \\
2.8 \\
3.4\end{array}$ & $\begin{array}{l}12.3 \\
23.2 \\
33.1 \\
43.0\end{array}$ & $\begin{array}{r}606 \\
1,187 \\
1,667 \\
2,071\end{array}$ & $\begin{array}{r}703 \\
1,373 \\
1,921 \\
2,387\end{array}$ & $\begin{array}{l}3.6 \\
2.1 \\
4.6 \\
7.4\end{array}$ \\
\hline $\begin{array}{l}\text { Average Number of Vehicles per } \\
\text { Household During the Year } \\
\text { Part-Year Vehicle } \\
\text { only } 1 \\
\text { Between } 1 \text { and } 2 \\
\text { Only } 2 \\
\text { Between } 2 \text { and } 3 \\
\text { Only } 3 \\
\text { Between } 3 \text { and } 4 \\
4 \text { or More }\end{array}$ & $\begin{array}{r}2.4 \\
28.6 \\
5.5 \\
28.4 \\
5.6 \\
8.4 \\
2.3 \\
3.4\end{array}$ & $\begin{array}{l}0.5 \\
1.0 \\
1.5 \\
2.0 \\
2.4 \\
3.0 \\
3.5 \\
4.4\end{array}$ & $\begin{array}{r}5.9 \\
10.7 \\
19.5 \\
23.2 \\
29.1 \\
32.6 \\
44.3 \\
50.0\end{array}$ & $\begin{array}{r}281 \\
518 \\
990 \\
1,158 \\
1,522 \\
1,726 \\
2,183 \\
2,571\end{array}$ & $\begin{array}{r}324 \\
605 \\
1,142 \\
1,341 \\
1,749 \\
1,991 \\
2,481 \\
2,972\end{array}$ & $\begin{array}{r}32.8 \\
3.7 \\
5.3 \\
2.4 \\
3.8 \\
3.9 \\
6.9 \\
4.6\end{array}$ \\
\hline
\end{tabular}

1 Below 150 percent of poverty line or 60 percent of median State income.

2 Approximately 0.5 percent of the vehicle stock was owned by households that had no drivers as of fall 1993.

Notes: - "Households with Children" category includes members under age 18 years old unless the member is the householder or spouse. • To obtain the Relative Standard Error (RSE) percentage for any table cell, multiply the corresponding column and row factors. - Because of rounding, data may not sum to totals. - Data in this table are for households with vehicles for personal transportation. - See Glossary for definition of terms used in this report.

Source: Energy Information Administration, Office of Energy Markets and End Use, Form ElA-457 A of the 1993 Residential Energy Consumption Survey and Forms EIA-876 A, B, C, and D of the 1994 Residential Transportation Energy Consumption Survey. 
Table 5.3. U.S. per Vehicle Miles Traveled, Vehicle Fuel Consumption and Expenditures, 1994

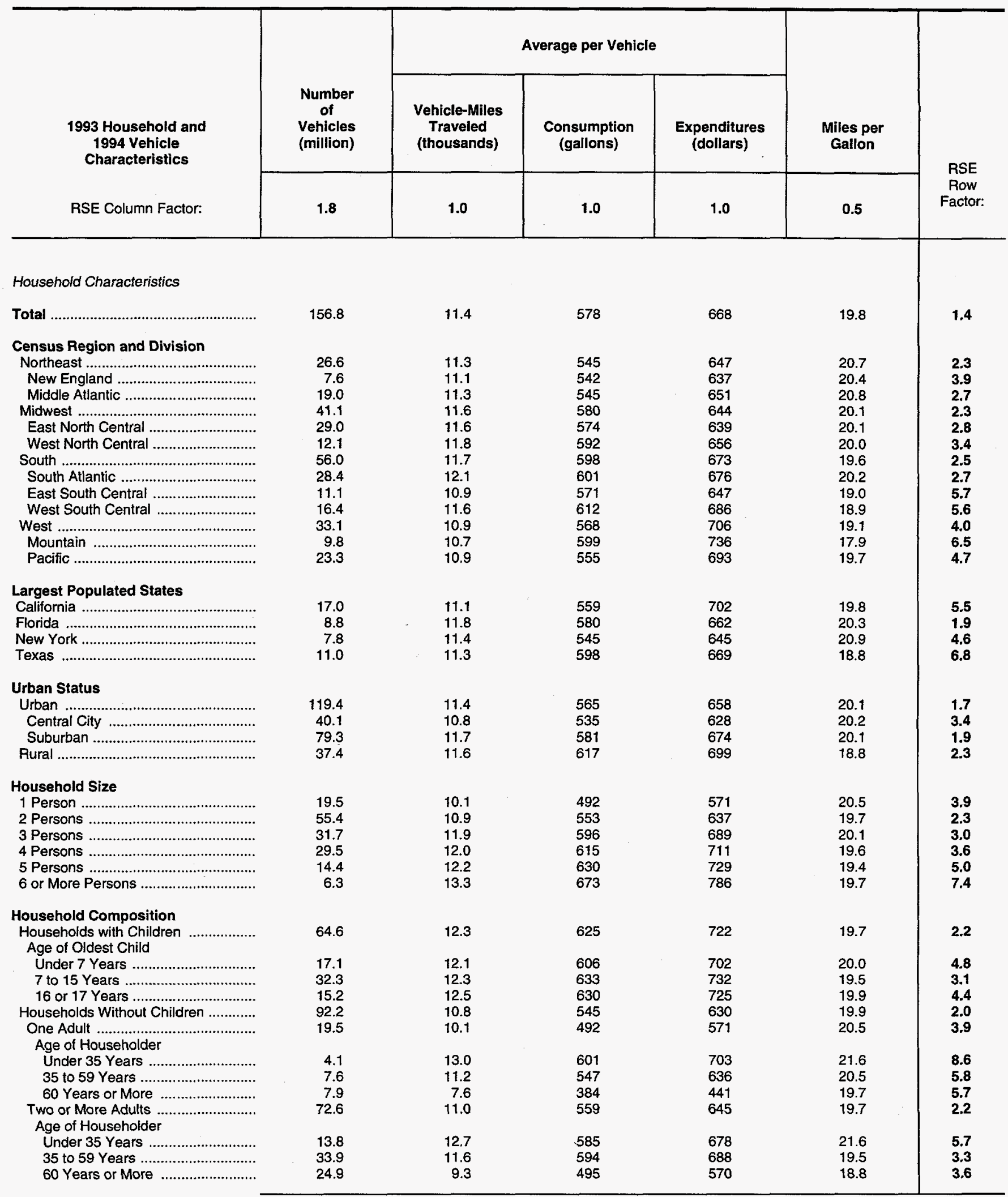

See footnotes at end of table. 
Table 5.3. U.S. per Vehicle Miles Traveled, Vehicle Fuel Consumption and Expenditures, 1994 (Continued)

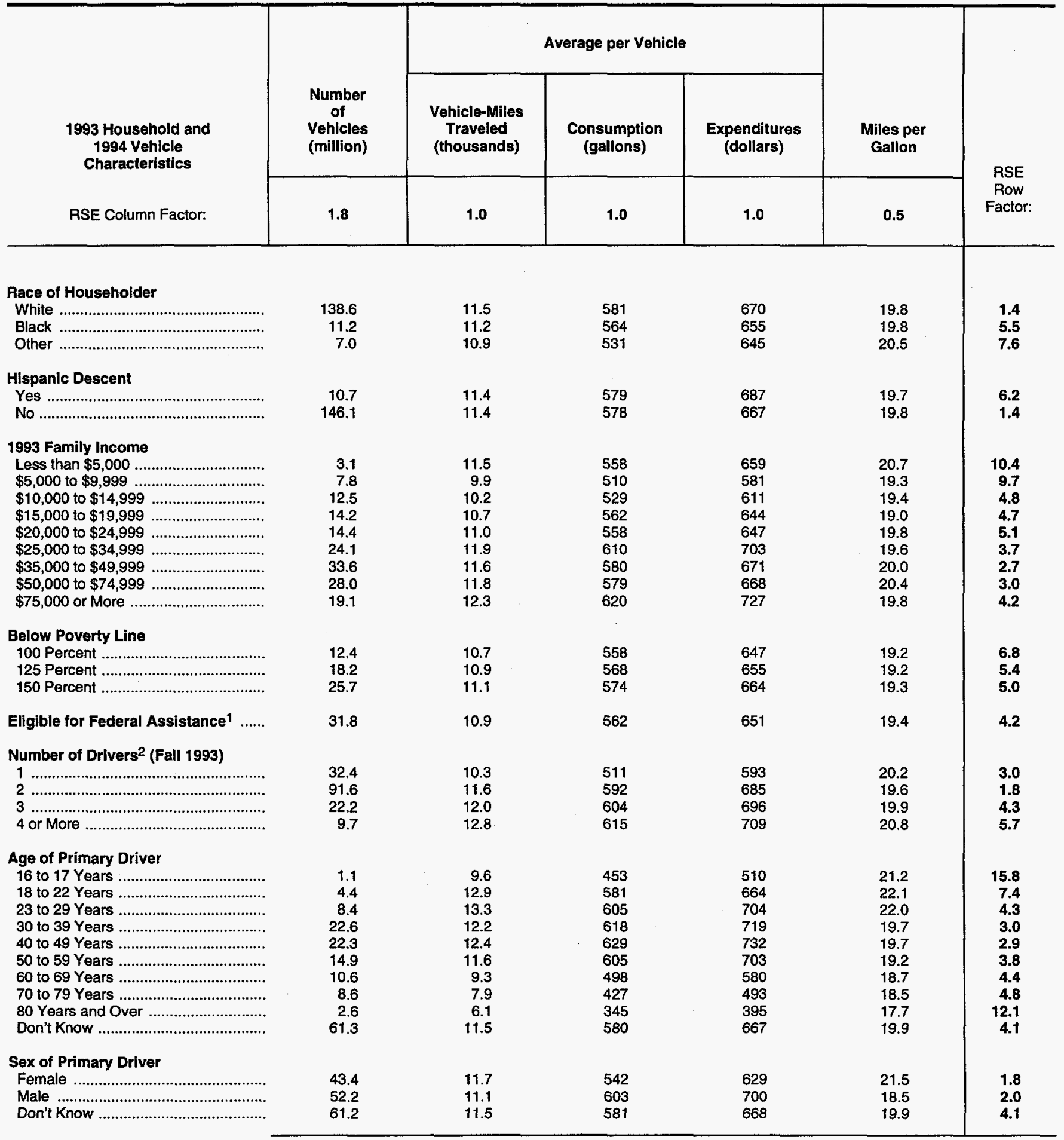

See footnotes at end of table. 
Table 5.3. U.S. per Vehicle Miles Traveled, Vehicle Fuel Consumption and Expenditures, 1994 (Continued)

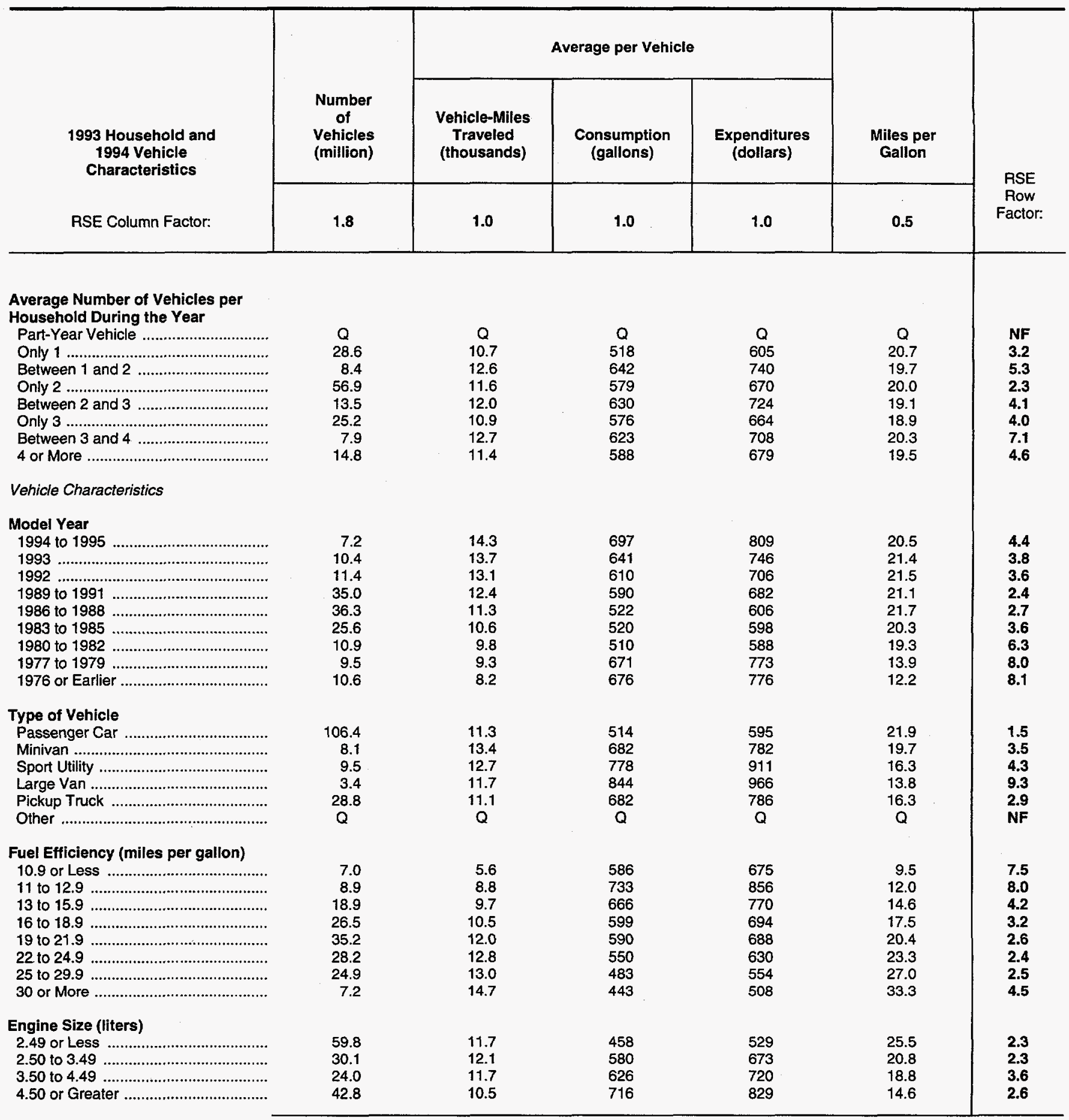

See footnotes at end of table. 
Table 5.3. U.S. per Vehicle Miles Traveled, Vehicle Fuel Consumption and Expenditures, 1994 (Continued)

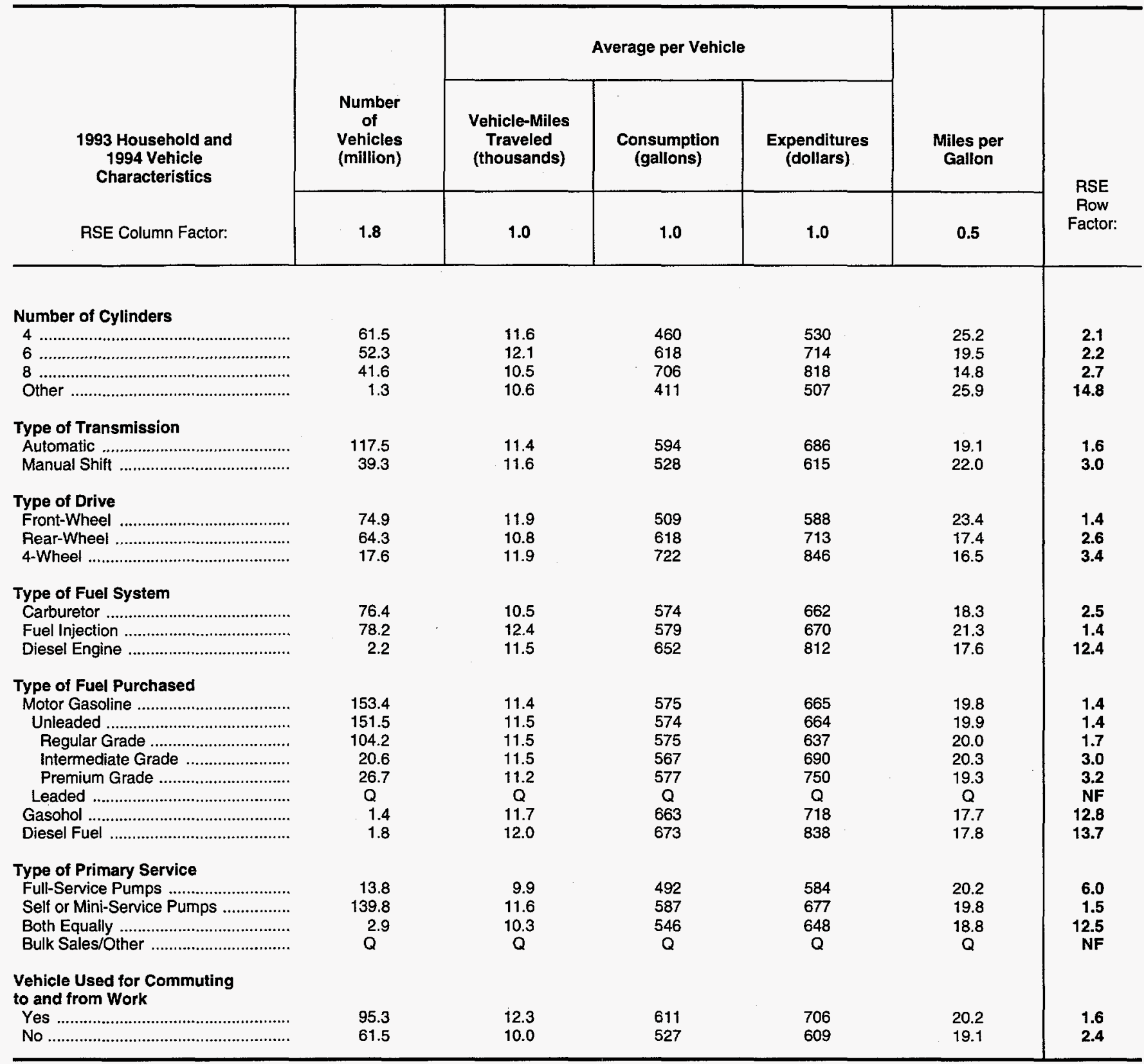

1 Below 150 percent of poverty line or 60 percent of median State income.

2 Approximately 0.5 percent of the vehicle stock was owned by households that had no drivers as of fall 1993.

NF = No applicable RSE row factor.

$Q=$ Data withheld either because the Relative Standard Error (RSE) was greater than 50 percent or fewer than 10 households were sampled.

Notes: "Households with Children" category includes members under age 18 years old unless the member is the householder or spouse. - To obtain the Relative Standard Error (RSE) percentage for any table cell, multiply the corresponding column and row factors. • Because of rounding, data may not sum to totals. - Data in this table are for households with vehicles for personal transportation. - See Glossary for definition of terms used in this report.

Source: Energy Information Administration, Office of Energy Markets and End Use, Form ElA-457 A of the 1993 Residential Energy Consumption Survey and Forms EIA-876 A, B, C, and D of the 1994 Residential Transportation Energy Consumption Survey. 


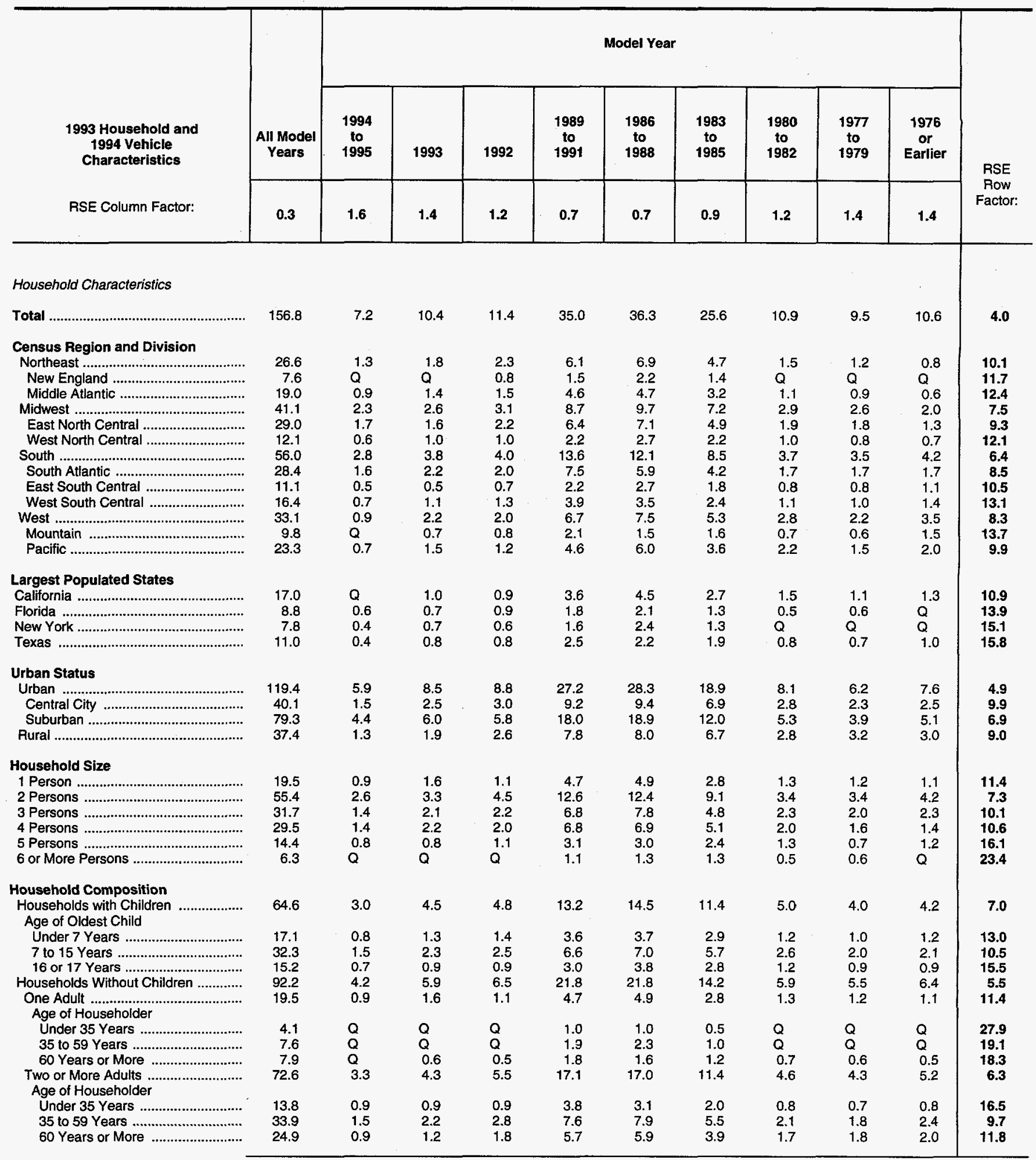

See footnotes at end of table. 
Table 5.4. U.S. Vehicles by Model Year, 1994 (Continued) (Million Vehicles)

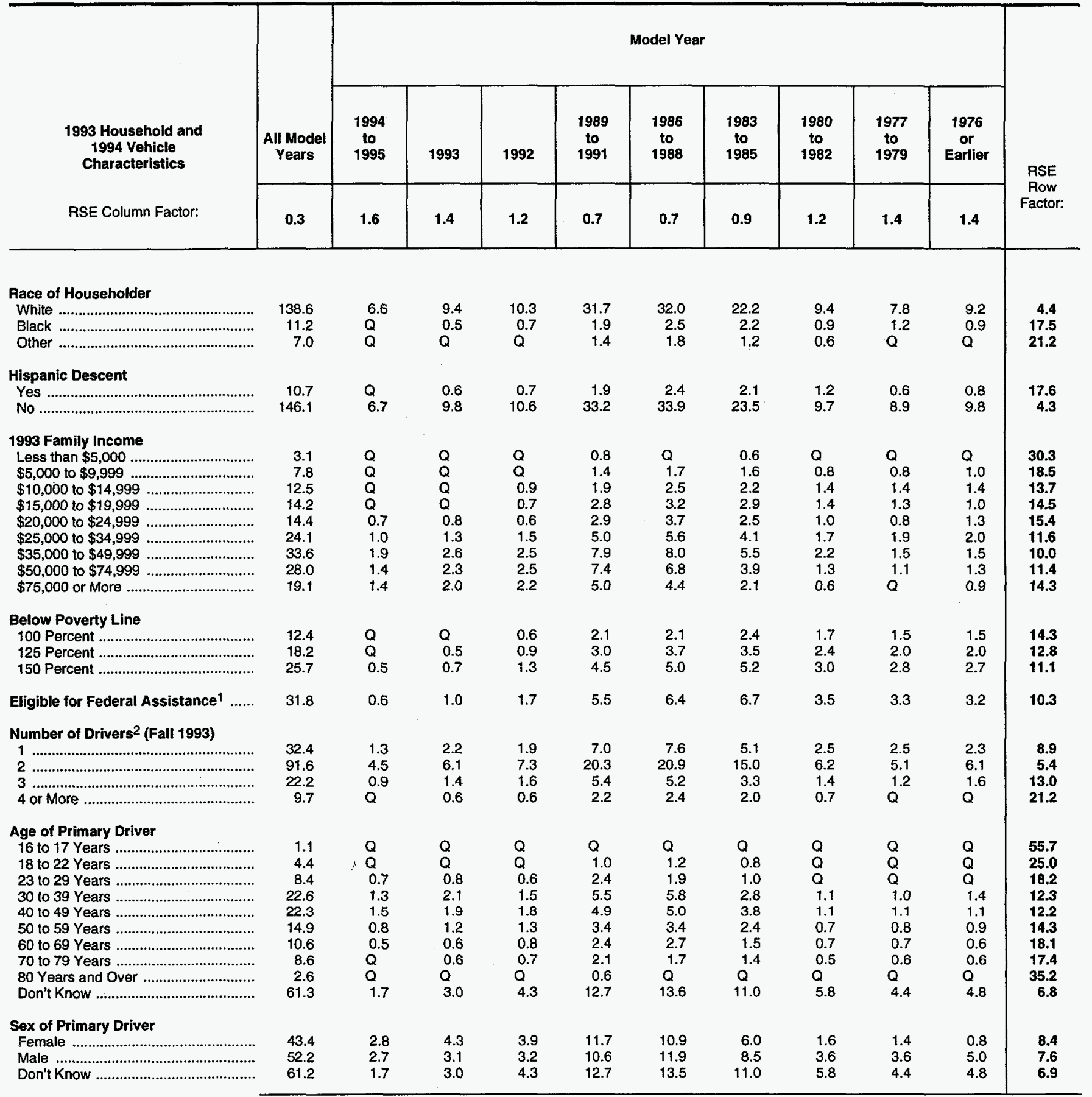

See footnotes at end of table. 
Table 5.4. U.S. Vehicles by Model Year, 1994 (Continued) (Million Vehicles)

\begin{tabular}{|c|c|c|c|c|c|c|c|c|c|c|c|}
\hline \multirow{3}{*}{$\begin{array}{c}1993 \text { Household and } \\
1994 \text { Vehicle } \\
\text { Characteristics }\end{array}$} & \multirow[b]{2}{*}{$\begin{array}{l}\text { All Model } \\
\text { Years }\end{array}$} & \multicolumn{9}{|c|}{ Model Year } & \multirow{3}{*}{$\begin{array}{c}\text { RSE } \\
\text { Row } \\
\text { Factor: }\end{array}$} \\
\hline & & $\begin{array}{c}1994 \\
10 \\
1995\end{array}$ & 1993 & 1992 & $\begin{array}{c}1989 \\
\text { to } \\
1991\end{array}$ & $\begin{array}{c}1986 \\
\text { to } \\
1988\end{array}$ & $\begin{array}{c}1983 \\
\text { to } \\
1985\end{array}$ & $\begin{array}{c}1980 \\
\text { to } \\
1982\end{array}$ & $\begin{array}{l}1977 \\
\text { to } \\
1979\end{array}$ & $\begin{array}{c}1976 \\
\text { or } \\
\text { Earlier }\end{array}$ & \\
\hline & 0.3 & 1.6 & 1.4 & 1.2 & 0.7 & 0.7 & 0.9 & 1.2 & 1.4 & 1.4 & \\
\hline \multicolumn{12}{|l|}{$\begin{array}{l}\text { Average Number of Vehicles per } \\
\text { Household During the Year }\end{array}$} \\
\hline 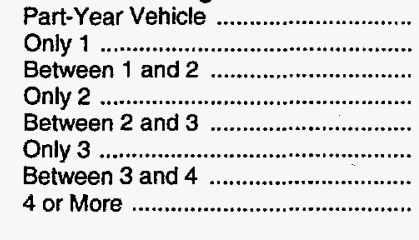 & $\begin{array}{r}1.3 \\
28.6 \\
8.4 \\
56.9 \\
13.5 \\
25.2 \\
7.9 \\
14.8\end{array}$ & $\begin{array}{l}Q \\
0.8 \\
0.9 \\
1.7 \\
1.7 \\
0.8 \\
0.6 \\
0.6\end{array}$ & $\begin{array}{l}Q \\
1.8 \\
0.8 \\
3.9 \\
1.0 \\
1.2 \\
0.6 \\
1.0\end{array}$ & $\begin{array}{l}Q \\
2.1 \\
0.5 \\
4.9 \\
0.7 \\
1.7 \\
0.6 \\
0.7\end{array}$ & $\begin{array}{r}Q \\
6.5 \\
1.7 \\
13.1 \\
2.9 \\
6.1 \\
1.6 \\
2.8\end{array}$ & $\begin{array}{r}0.3 \\
6.9 \\
1.4 \\
14.8 \\
2.8 \\
5.4 \\
1.9 \\
2.9\end{array}$ & $\begin{array}{l}0.3 \\
5.0 \\
1.4 \\
8.6 \\
1.9 \\
4.0 \\
1.5 \\
2.8\end{array}$ & $\begin{array}{l}Q \\
2.0 \\
0.6 \\
3.7 \\
0.8 \\
2.1 \\
0.4 \\
1.1\end{array}$ & $\begin{array}{l}Q \\
1.9 \\
0.6 \\
3.0 \\
0.9 \\
1.5 \\
0.5 \\
1.0\end{array}$ & $\begin{array}{l}Q \\
1.5 \\
0.6 \\
3.2 \\
0.9 \\
2.2 \\
Q \\
2.0\end{array}$ & $\begin{array}{r}34.4 \\
9.6 \\
17.1 \\
7.7 \\
15.3 \\
12.1 \\
21.6 \\
17.6\end{array}$ \\
\hline \multicolumn{12}{|l|}{ Vehicle Characteristics } \\
\hline $\begin{array}{l}\text { Type of Vehicle } \\
\text { Passenger Car } \\
\text { Minivan } \\
\text { Sport Utility } \\
\text { Large Van } \\
\text { Pickup Truck } \\
\text { Other }\end{array}$ & $\begin{array}{r}106.4 \\
8.1 \\
9.5 \\
3.4 \\
28.8 \\
0.6\end{array}$ & $\begin{array}{l}4.1 \\
0.8 \\
0.8 \\
Q \\
1.5 \\
Q\end{array}$ & $\begin{array}{l}6.9 \\
1.0 \\
1.0 \\
Q \\
1.4 \\
Q\end{array}$ & $\begin{array}{l}7.8 \\
0.8 \\
1.0 \\
Q \\
1.6 \\
Q\end{array}$ & $\begin{array}{r}23.6 \\
2.9 \\
2.5 \\
0.7 \\
5.2 \\
Q\end{array}$ & $\begin{array}{l}26.1 \\
1.9 \\
2.0 \\
0.7 \\
5.6 \\
Q\end{array}$ & $\begin{array}{r}18.2 \\
0.5 \\
1.1 \\
0.8 \\
4.7 \\
Q\end{array}$ & $\begin{array}{l}8.1 \\
Q \\
Q \\
Q \\
2.1 \\
Q\end{array}$ & $\begin{array}{l}5.6 \\
Q \\
Q \\
Q \\
3.2 \\
Q\end{array}$ & $\begin{array}{l}6.0 \\
Q \\
0.6 \\
Q \\
3.5 \\
Q\end{array}$ & $\begin{array}{r}4.8 \\
15.4 \\
15.6 \\
29.5 \\
9.1 \\
96.7\end{array}$ \\
\hline 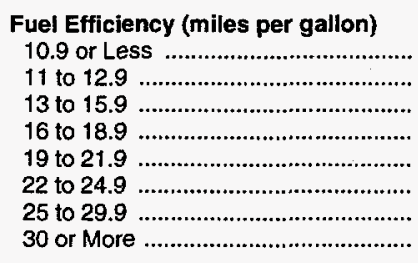 & $\begin{array}{r}7.0 \\
8.9 \\
18.9 \\
26.5 \\
35.2 \\
28.2 \\
24.9 \\
7.2\end{array}$ & $\begin{array}{l}Q \\
Q \\
0.9 \\
1.4 \\
1.8 \\
1.5 \\
1.0 \\
Q\end{array}$ & $\begin{array}{l}Q \\
Q \\
0.7 \\
1.8 \\
3.2 \\
2.2 \\
1.5 \\
0.8\end{array}$ & $\begin{array}{l}Q \\
Q \\
0.8 \\
2.2 \\
3.0 \\
2.6 \\
2.1 \\
0.6\end{array}$ & $\begin{array}{l}Q \\
1.0 \\
2.9 \\
5.3 \\
8.7 \\
9.1 \\
6.4 \\
1.5\end{array}$ & $\begin{array}{l}Q \\
1.1 \\
3.0 \\
4.8 \\
9.6 \\
8.0 \\
7.5 \\
2.1\end{array}$ & $\begin{array}{l}Q \\
1.2 \\
2.9 \\
6.0 \\
5.6 \\
3.3 \\
4.7 \\
1.4\end{array}$ & $\begin{array}{l}Q \\
0.6 \\
2.0 \\
2.6 \\
2.3 \\
1.2 \\
1.6 \\
Q\end{array}$ & $\begin{array}{l}1.7 \\
2.2 \\
3.3 \\
1.5 \\
Q \\
Q \\
Q \\
Q\end{array}$ & $\begin{array}{l}4.0 \\
2.6 \\
2.3 \\
0.9 \\
Q \\
Q \\
Q \\
Q\end{array}$ & $\begin{array}{r}10.6 \\
16.3 \\
11.6 \\
10.4 \\
8.4 \\
10.0 \\
10.0 \\
18.8\end{array}$ \\
\hline 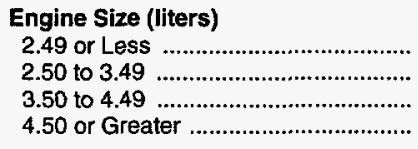 & $\begin{array}{l}59.8 \\
30.1 \\
24.0 \\
42.8\end{array}$ & $\begin{array}{l}2.1 \\
1.7 \\
1.6 \\
1.7\end{array}$ & $\begin{array}{l}3.7 \\
3.2 \\
2.0 \\
1.4\end{array}$ & $\begin{array}{l}4.3 \\
3.3 \\
2.3 \\
1.5\end{array}$ & $\begin{array}{r}14.7 \\
8.7 \\
5.6 \\
6.1\end{array}$ & $\begin{array}{r}17.6 \\
7.7 \\
4.5 \\
6.5\end{array}$ & $\begin{array}{r}11.0 \\
3.6 \\
3.5 \\
7.5\end{array}$ & $\begin{array}{l}4.5 \\
1.1 \\
2.3 \\
3.0\end{array}$ & $\begin{array}{l}1.0 \\
0.5 \\
1.3 \\
6.6\end{array}$ & $\begin{array}{l}0.9 \\
Q \\
1.0 \\
8.4\end{array}$ & $\begin{array}{r}7.8 \\
10.0 \\
11.1 \\
8.1\end{array}$ \\
\hline Number of Cylinders & $\begin{array}{r}61.5 \\
52.3 \\
41.6 \\
1.3\end{array}$ & $\begin{array}{l}2.1 \\
3.4 \\
1.6 \\
Q\end{array}$ & $\begin{array}{l}3.7 \\
5.2 \\
1.3 \\
Q\end{array}$ & $\begin{array}{l}4.4 \\
5.5 \\
1.4 \\
Q\end{array}$ & $\begin{array}{c}15.3 \\
13.3 \\
6.1 \\
Q\end{array}$ & $\begin{array}{c}18.3 \\
11.3 \\
6.4 \\
Q\end{array}$ & $\begin{array}{l}11.0 \\
6.7 \\
7.6 \\
Q\end{array}$ & $\begin{array}{l}4.4 \\
2.9 \\
3.4 \\
Q\end{array}$ & $\begin{array}{l}1.0 \\
1.8 \\
6.7 \\
Q\end{array}$ & $\begin{array}{l}1.3 \\
2.1 \\
7.1 \\
Q\end{array}$ & $\begin{array}{r}7.4 \\
6.9 \\
8.2 \\
60.0\end{array}$ \\
\hline 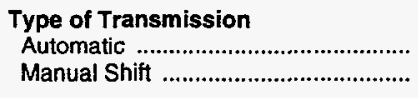 & $\begin{array}{r}117.5 \\
39.3\end{array}$ & $\begin{array}{l}5.6 \\
1.6\end{array}$ & $\begin{array}{l}8.1 \\
2.2\end{array}$ & $\begin{array}{l}8.9 \\
2.5\end{array}$ & $\begin{array}{r}27.5 \\
7.5\end{array}$ & $\begin{array}{r}27.0 \\
9.3\end{array}$ & $\begin{array}{r}18.7 \\
6.9\end{array}$ & $\begin{array}{l}7.1 \\
3.8\end{array}$ & $\begin{array}{l}7.4 \\
2.0\end{array}$ & $\begin{array}{l}7.1 \\
3.5\end{array}$ & $\begin{array}{l}4.6 \\
8.7\end{array}$ \\
\hline $\begin{array}{l}\text { Type of Drive } \\
\text { Front-Wheel } \\
\text { Rear-Wheel } \\
\text { 4-Wheel }\end{array}$ & $\begin{array}{l}74.9 \\
64.3 \\
17.6\end{array}$ & $\begin{array}{l}4.3 \\
1.7 \\
1.2\end{array}$ & $\begin{array}{l}6.2 \\
2.4 \\
1.8\end{array}$ & $\begin{array}{l}7.2 \\
2.7 \\
1.4\end{array}$ & $\begin{array}{r}21.0 \\
9.9 \\
4.1\end{array}$ & $\begin{array}{r}19.9 \\
12.7 \\
3.6\end{array}$ & $\begin{array}{r}11.0 \\
12.0 \\
2.6\end{array}$ & $\begin{array}{l}3.3 \\
6.7 \\
0.9\end{array}$ & $\begin{array}{l}0.8 \\
7.6 \\
1.0\end{array}$ & $\begin{array}{l}1.1 \\
8.6 \\
0.9\end{array}$ & $\begin{array}{r}6.4 \\
6.7 \\
12.5\end{array}$ \\
\hline $\begin{array}{l}\text { Type of Fuel System } \\
\text { Carburetor } \\
\text { Fuel Injection } \\
\text { Diesel Engine }\end{array}$ & $\begin{array}{r}76.4 \\
78.2 \\
2.2\end{array}$ & $\begin{array}{l}1.1 \\
5.9 \\
Q\end{array}$ & $\begin{array}{l}1.4 \\
8.8 \\
Q\end{array}$ & $\begin{array}{l}1.7 \\
9.6 \\
Q\end{array}$ & $\begin{array}{l}7.1 \\
27.7 \\
Q\end{array}$ & $\begin{array}{l}17.8 \\
18.3 \\
Q\end{array}$ & $\begin{array}{r}18.7 \\
6.2 \\
0.7\end{array}$ & $\begin{array}{l}9.7 \\
0.7 \\
0.5\end{array}$ & $\begin{array}{l}8.8 \\
0.6 \\
Q\end{array}$ & $\begin{array}{l}10.1 \\
Q \\
Q\end{array}$ & $\begin{array}{r}6.3 \\
6.9 \\
27.8\end{array}$ \\
\hline
\end{tabular}

See footnotes at end of table. 
Table 5.4. U.S. Vehicles by Model Year, 1994 (Continued) (Million Vehicles)

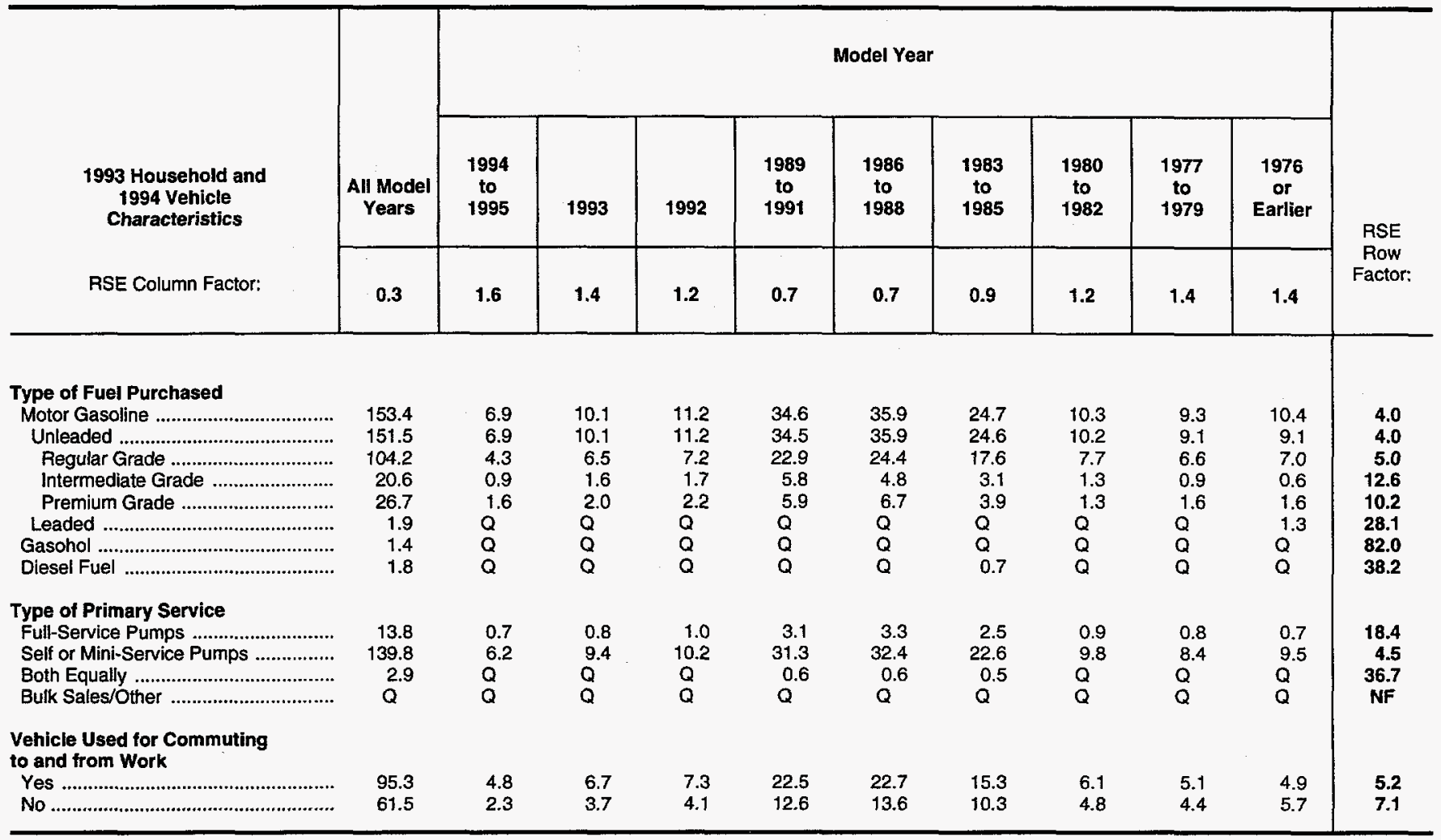

1 Below 150 percent of poverty line or 60 percent of median State income.

2 Approximately 0.5 percent of the vehicle stock was owned by households that had no drivers as of fall 1993.

NF = No applicable RSE row factor.

$\mathrm{Q}=$ Data withheld either because the Relative Standard Error (RSE) was greater than 50 percent or fewer than 10 households were sampled.

Notes: "Households with Children" category includes members under age 18 years old unless the member is the householder or spouse. - To obtain the Relative Standard Error (RSE) percentage for any table cell, multiply the corresponding column and row factors. - Because of rounding, data may not sum to totals. - Data in this table are for households with vehicles for personal transportation. - See Glossary for definition of terms used in this report.

Source: Energy Information Administration, Office of Energy Markets and End Use, Form EIA-457 A of the 1993 Residential Energy Consumption Survey and Forms ElA-B76 A, B, C, and D of the 1994 Residential Transportation Energy Consumption Survey. 
Table 5.5. U.S. Vehicle Fuel Efficiency by Model Year, 1994

(Miles per Gallon)

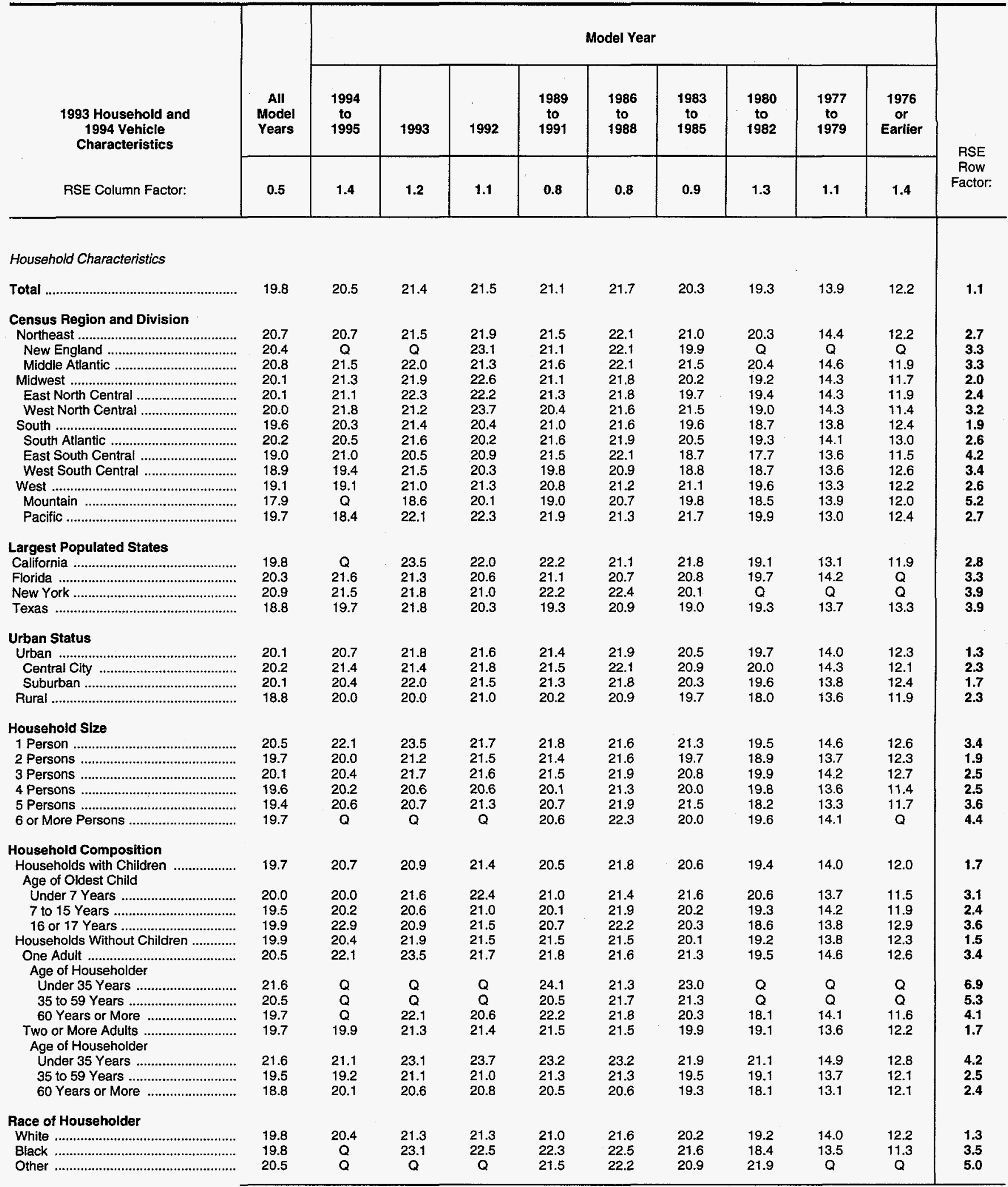

See footnotes at end of table. 
Table 5.5. U.S. Vehicle Fuel Efficiency by Model Year, 1994 (Continued) (Miles per Gallon)

\begin{tabular}{|c|c|c|c|c|c|c|c|c|c|c|c|}
\hline \multirow[b]{2}{*}{$\begin{array}{l}1993 \text { Household and } \\
1994 \text { Vehicle } \\
\text { Characteristics }\end{array}$} & \multirow[b]{2}{*}{$\begin{array}{l}\text { All } \\
\text { Model } \\
\text { Years }\end{array}$} & \multicolumn{9}{|c|}{ Model Year } & \multirow[b]{2}{*}{$\begin{array}{l}\text { RSE } \\
\text { Row } \\
\text { Factor: }\end{array}$} \\
\hline & & $\begin{array}{c}1994 \\
\text { to } \\
1995\end{array}$ & 1993 & 1992 & $\begin{array}{c}1989 \\
\text { to } \\
1991\end{array}$ & $\begin{array}{c}1986 \\
\text { to } \\
1988\end{array}$ & $\begin{array}{c}1983 \\
\text { to } \\
1985\end{array}$ & $\begin{array}{c}1980 \\
\text { to } \\
1982\end{array}$ & $\begin{array}{c}1977 \\
\text { to } \\
1979\end{array}$ & $\begin{array}{c}1976 \\
\text { or } \\
\text { Earlier }\end{array}$ & \\
\hline 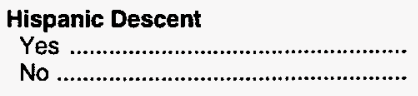 & $\begin{array}{l}19.7 \\
19.8\end{array}$ & $\underset{20.4}{Q}$ & $\begin{array}{l}21.0 \\
21.5\end{array}$ & $\begin{array}{l}21.7 \\
21.4\end{array}$ & $\begin{array}{l}20.7 \\
21.1\end{array}$ & $\begin{array}{l}21.7 \\
21.7\end{array}$ & $\begin{array}{l}20.1 \\
20.3\end{array}$ & $\begin{array}{l}19.7 \\
19.2\end{array}$ & $\begin{array}{l}13.3 \\
13.9\end{array}$ & $\begin{array}{l}13.4 \\
12.1\end{array}$ & $\begin{array}{l}4.1 \\
1.2\end{array}$ \\
\hline 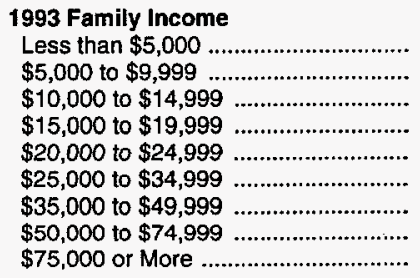 & $\begin{array}{l}20.7 \\
19.3 \\
19.4 \\
19.0 \\
19.8 \\
19.6 \\
20.0 \\
20.4 \\
19.8\end{array}$ & $\begin{array}{c}Q \\
Q \\
Q \\
Q \\
20.6 \\
21.5 \\
21.0 \\
19.6 \\
19.6\end{array}$ & $\begin{array}{c}Q \\
Q \\
Q \\
Q \\
23.2 \\
21.4 \\
22.4 \\
21.3 \\
20.2\end{array}$ & $\begin{array}{c}Q \\
Q \\
24.2 \\
22.1 \\
21.5 \\
21.6 \\
21.1 \\
21.8 \\
20.1\end{array}$ & $\begin{array}{l}23.2 \\
24.0 \\
22.7 \\
19.9 \\
21.6 \\
20.9 \\
20.5 \\
21.4 \\
20.6\end{array}$ & $\begin{array}{c}Q \\
23.5 \\
21.7 \\
22.2 \\
22.5 \\
21.8 \\
21.1 \\
21.8 \\
20.8\end{array}$ & $\begin{array}{l}23.9 \\
21.5 \\
21.1 \\
19.6 \\
20.5 \\
20.2 \\
20.1 \\
20.1 \\
19.6\end{array}$ & $\begin{array}{c}Q \\
17.7 \\
20.1 \\
19.8 \\
19.6 \\
19.3 \\
18.8 \\
18.5 \\
20.2\end{array}$ & $\begin{array}{c}Q \\
13.5 \\
13.4 \\
13.6 \\
13.4 \\
14.7 \\
13.9 \\
13.9 \\
Q\end{array}$ & $\begin{array}{c}Q \\
11.9 \\
12.4 \\
11.8 \\
12.2 \\
12.5 \\
12.4 \\
11.8 \\
11.6\end{array}$ & $\begin{array}{l}8.5 \\
4.7 \\
3.4 \\
3.0 \\
3.9 \\
2.8 \\
2.6 \\
2.7 \\
3.2\end{array}$ \\
\hline 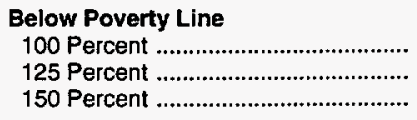 & $\begin{array}{l}19.2 \\
19.2 \\
19.3\end{array}$ & $\begin{array}{c}Q \\
Q \\
21.6\end{array}$ & $\begin{array}{c}Q \\
20.2 \\
21.0\end{array}$ & $\begin{array}{l}22.7 \\
22.7 \\
23.6\end{array}$ & $\begin{array}{l}22.4 \\
22.2 \\
22.0\end{array}$ & $\begin{array}{l}22.0 \\
22.4 \\
22.8\end{array}$ & $\begin{array}{l}22.4 \\
21.4 \\
20.8\end{array}$ & $\begin{array}{l}20.1 \\
20.1 \\
19.9\end{array}$ & $\begin{array}{l}13.4 \\
13.9 \\
13.6\end{array}$ & $\begin{array}{l}12.4 \\
12.2 \\
11.9\end{array}$ & $\begin{array}{l}3.4 \\
3.1 \\
2.9\end{array}$ \\
\hline Eligible for Federal Assistance ${ }^{1} \ldots . .$. & 19.4 & 21.6 & 21.4 & 23.1 & 22.1 & 22.4 & 21.1 & 19.6 & 13.6 & 11.9 & 2.5 \\
\hline 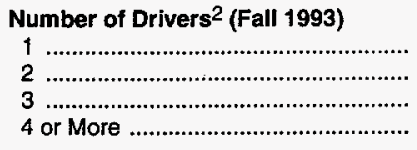 & $\begin{array}{l}20.2 \\
19.6 \\
19.9 \\
20.8\end{array}$ & $\begin{array}{c}21.7 \\
19.8 \\
22.6 \\
Q\end{array}$ & $\begin{array}{l}22.8 \\
20.9 \\
21.2 \\
23.1\end{array}$ & $\begin{array}{l}21.7 \\
21.4 \\
21.2 \\
22.8\end{array}$ & $\begin{array}{l}21.6 \\
20.8 \\
21.2 \\
21.7\end{array}$ & $\begin{array}{l}22.1 \\
21.4 \\
22.1 \\
22.1\end{array}$ & $\begin{array}{l}21.3 \\
19.9 \\
20.4 \\
21.1\end{array}$ & $\begin{array}{c}19.7 \\
19.1 \\
19.4 \\
19.0\end{array}$ & $\begin{array}{c}14.0 \\
13.9 \\
13.3 \\
\mathbf{Q}\end{array}$ & $\begin{array}{c}12.8 \\
11.9 \\
12.6 \\
Q\end{array}$ & $\begin{array}{l}2.4 \\
1.5 \\
3.2 \\
4.6\end{array}$ \\
\hline 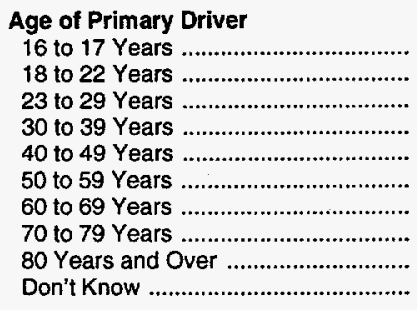 & $\begin{array}{l}21.2 \\
22.1 \\
22.0 \\
19.7 \\
19.7 \\
19.2 \\
18.7 \\
18.5 \\
17.7 \\
19.9\end{array}$ & $\begin{array}{l}Q \\
Q \\
22.0 \\
20.7 \\
20.0 \\
19.9 \\
20.6 \\
Q \\
Q \\
20.4\end{array}$ & $\begin{array}{c}Q \\
Q \\
24.0 \\
21.8 \\
20.2 \\
21.5 \\
20.4 \\
21.1 \\
Q \\
21.4\end{array}$ & $\begin{array}{c}Q \\
Q \\
25.3 \\
21.9 \\
20.5 \\
21.9 \\
20.4 \\
21.5 \\
Q \\
21.1\end{array}$ & $\begin{array}{c}Q \\
24.2 \\
22.9 \\
20.5 \\
20.6 \\
20.2 \\
20.4 \\
19.5 \\
21.2 \\
21.5\end{array}$ & $\begin{array}{c}Q \\
24.9 \\
22.4 \\
21.7 \\
22.0 \\
19.9 \\
20.1 \\
20.4 \\
Q \\
21.9\end{array}$ & $\begin{array}{c}Q \\
22.4 \\
21.6 \\
19.5 \\
18.9 \\
19.6 \\
18.6 \\
18.6 \\
Q \\
21.3\end{array}$ & $\begin{array}{c}Q \\
Q \\
Q \\
18.5 \\
18.1 \\
18.5 \\
18.3 \\
18.3 \\
Q \\
19.9\end{array}$ & $\begin{array}{c}\mathbf{Q} \\
\mathbf{Q} \\
\mathbf{Q} \\
13.3 \\
14.6 \\
13.6 \\
13.0 \\
12.4 \\
\mathbf{Q} \\
14.2\end{array}$ & $\begin{array}{c}Q \\
Q \\
Q \\
11.5 \\
12.5 \\
11.7 \\
10.4 \\
11.3 \\
Q \\
12.6\end{array}$ & $\begin{array}{l}9.2 \\
5.2 \\
4.2 \\
3.4 \\
3.0 \\
3.4 \\
4.3 \\
4.0 \\
6.6 \\
1.9\end{array}$ \\
\hline $\begin{array}{l}\text { Sex of Primary Driver } \\
\text { Female } \\
\text { Male } \\
\text { Don't Know }\end{array}$ & $\begin{array}{l}21.5 \\
18.5 \\
19.9\end{array}$ & $\begin{array}{l}22.4 \\
18.9 \\
20.4\end{array}$ & $\begin{array}{l}22.2 \\
20.6 \\
21.4\end{array}$ & $\begin{array}{l}22.4 \\
20.8 \\
21.1\end{array}$ & $\begin{array}{l}22.2 \\
19.6 \\
21.5\end{array}$ & $\begin{array}{l}22.3 \\
20.9 \\
21.9\end{array}$ & $\begin{array}{l}20.8 \\
18.7 \\
21.3\end{array}$ & $\begin{array}{l}18.4 \\
18.5 \\
19.9\end{array}$ & $\begin{array}{l}14.9 \\
13.1 \\
14.2\end{array}$ & $\begin{array}{l}12.5 \\
11.7 \\
12.6\end{array}$ & $\begin{array}{l}2.0 \\
2.0 \\
1.9\end{array}$ \\
\hline
\end{tabular}

See footnotes at end of table. 
Table 5.5. U.S. Vehicle Fuel Efficiency by Model Year, 1994 (Continued) (Miles per Gallon)

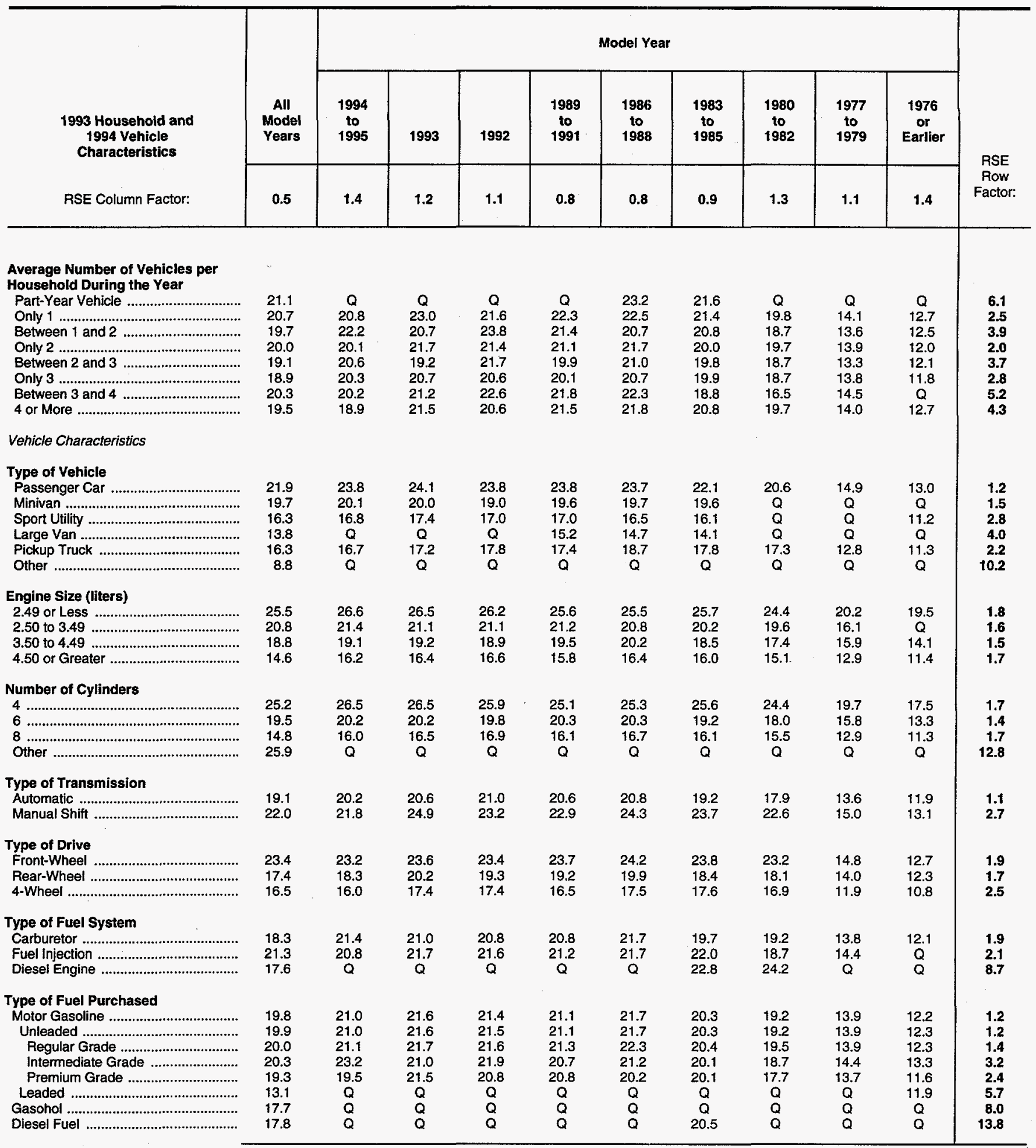

See footnotes at end of table. 
Table 5.5. U.S. Vehicle Fuel Efficiency by Model Year, 1994 (Continued) (Miles per Gallon)

\begin{tabular}{|c|c|c|c|c|c|c|c|c|c|c|c|}
\hline \multirow{3}{*}{$\begin{array}{c}1993 \text { Household and } \\
1994 \text { Vehicle } \\
\text { Characteristics }\end{array}$} & \multirow[b]{2}{*}{$\begin{array}{l}\text { All } \\
\text { Model } \\
\text { Years }\end{array}$} & \multicolumn{9}{|c|}{ Model Year } & \multirow{3}{*}{$\begin{array}{l}\text { RSE } \\
\text { Row } \\
\text { Factor: }\end{array}$} \\
\hline & & $\begin{array}{l}1994 \\
\text { to } \\
1995\end{array}$ & 1993 & 1992 & $\begin{array}{c}1989 \\
\text { to } \\
1991\end{array}$ & $\begin{array}{c}1986 \\
\text { to } \\
1988\end{array}$ & $\begin{array}{l}1983 \\
\text { to } \\
1985\end{array}$ & $\begin{array}{c}1980 \\
\text { to } \\
1982\end{array}$ & $\begin{array}{c}1977 \\
\text { to } \\
1979\end{array}$ & $\begin{array}{c}1976 \\
\text { or } \\
\text { Earlier }\end{array}$ & \\
\hline & 0.5 & 1.4 & 1.2 & 1.1 & 0.8 & 0.8 & 0.9 & 1.3 & 1.1 & 1.4 & \\
\hline \multicolumn{12}{|l|}{ Type of Primary Service } \\
\hline 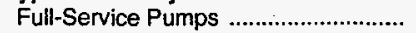 & 20.2 & 22.4 & 22.1 & 21.3 & 21.3 & 21.7 & 21.4 & 20.2 & 13.5 & 11.3 & 3.4 \\
\hline Self or Mini-Service Pumps ............... & 19.8 & 20.3 & 21.4 & 21.4 & 21.1 & 21.7 & 20.2 & 19.3 & 13.9 & 12.3 & 1.2 \\
\hline $\begin{array}{l}\text { Both Equally } \\
\text { Bulk Sales/Other }\end{array}$ & $\begin{array}{c}18.8 \\
Q\end{array}$ & $\begin{array}{l}Q \\
Q\end{array}$ & $\begin{array}{l}\mathbf{Q} \\
\mathbf{Q}\end{array}$ & $\mathbf{Q}$ & $\begin{array}{c}19.6 \\
Q\end{array}$ & $\begin{array}{c}19.9 \\
Q\end{array}$ & $\begin{array}{c}20.7 \\
Q\end{array}$ & $\begin{array}{l}Q \\
Q\end{array}$ & $\begin{array}{l}\mathbf{Q} \\
\mathbf{Q}\end{array}$ & $\begin{array}{l}Q \\
Q\end{array}$ & $\begin{array}{l}8.0 \\
\text { NF }\end{array}$ \\
\hline \multicolumn{12}{|l|}{$\begin{array}{l}\text { Vehicle Used for Commuting } \\
\text { to and from Work }\end{array}$} \\
\hline 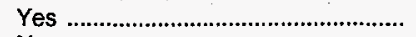 & 20.2 & 20.7 & 21.9 & 21.7 & 21.4 & 21.9 & 20.6 & 19.2 & 14.0 & 12.1 & 1.4 \\
\hline 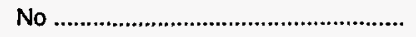 & 19.1 & 20.1 & 20.5 & 20.9 & 20.5 & 21.3 & 19.9 & 19.4 & 13.7 & 12.3 & 1.9 \\
\hline
\end{tabular}

1 Below 150 percent of poverty line or 60 percent of median State income.

2 Approximately 0.5 percent of the vehicle stock was owned by households that had no drivers as of fall 1993.

NF = No applicable RSE row factor.

$\mathrm{Q}=$ Data withheld either because the Relative Standard Error (RSE) was greater than 50 percent or fewer than 10 households were sampled.

Notes: "Households with Children" category includes members under age 18 years oid unless the member is the householder or spouse. - To obtain the Relative Standard Error (RSE) percentage for any table cell, multiply the corresponding column and row factors. - Because of rounding, data may not sum to totals. - Data in this table are for households with vehicles for personal transportation. - See Glossary for definition of terms used in this report.

Source: Energy Information Administration, Office of Energy Markets and End Use, Form ElA-457 A of the 1993 Residential Energy Consumption Survey and Forms ElA-876 A, B, C, and D of the 1994 Residential Transportation Energy Consumption Survey. 
Table 5.6. U.S. Average Vehicle Fuel Consumption by Model Year, 1994 (Gallons per Vehicle)

\begin{tabular}{|c|c|c|c|c|c|c|c|c|c|c|c|}
\hline \multirow{3}{*}{$\begin{array}{c}1993 \text { Household and } \\
1994 \text { Vehicle } \\
\text { Characteristics }\end{array}$} & \multirow[b]{2}{*}{$\begin{array}{c}\text { All } \\
\text { Model } \\
\text { Years }\end{array}$} & \multicolumn{9}{|c|}{ Model Year } & \multirow{3}{*}{$\begin{array}{l}\text { RSE } \\
\text { Row } \\
\text { Factor: }\end{array}$} \\
\hline & & $\begin{array}{c}1994 \\
\text { to } \\
1995\end{array}$ & 1993 & 1992 & $\begin{array}{l}1989 \\
\text { to } \\
1991\end{array}$ & $\begin{array}{c}1986 \\
\text { to } \\
1988\end{array}$ & $\begin{array}{c}1983 \\
\text { to } \\
1985\end{array}$ & $\begin{array}{l}1980 \\
\text { to } \\
1982\end{array}$ & $\begin{array}{c}1977 \\
\text { to } \\
1979\end{array}$ & $\begin{array}{c}1976 \\
\text { or } \\
\text { Earlier }\end{array}$ & \\
\hline & 0.6 & 0.9 & 0.8 & 0.8 & 1.4 & 0.6 & 0.8 & 1.4 & 1.8 & 1.9 & \\
\hline \multicolumn{12}{|l|}{ Household Characteristics } \\
\hline Total & 578 & 697 & 641 & 610 & 541 & 522 & 520 & 510 & 671 & 676 & 5.1 \\
\hline \multicolumn{12}{|l|}{ Census Region and Division } \\
\hline 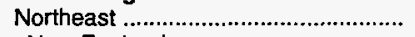 & 545 & 693 & 588 & 584 & 546 & 477 & 502 & 468 & $Q$ & $Q$ & 10.2 \\
\hline 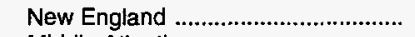 & 542 & $Q$ & $Q$ & $Q$ & 622 & 418 & $Q$ & $Q$ & $\overline{\mathbf{Q}}$ & $Q$ & 16.0 \\
\hline Middle Atlantic ..................................... & 545 & 618 & 569 & 591 & 522 & 504 & 500 & 465 & $\vec{Q}$ & $\mathbf{Q}$ & 12.1 \\
\hline 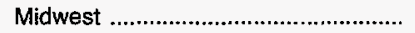 & 580 & 657 & 671 & 615 & 596 & 531 & 525 & 463 & 657 & 775 & 8.3 \\
\hline 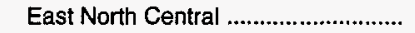 & 574 & 654 & 634 & 620 & 567 & 516 & 545 & 452 & 690 & $Q$ & 10.1 \\
\hline 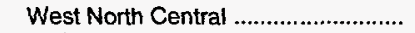 & 592 & 668 & 735 & 603 & 680 & 571 & 483 & $\mathbf{Q}$ & Q & $\bar{Q}$ & 12.2 \\
\hline South & 598 & 733 & 653 & 628 & 485 & 543 & 561 & 538 & 653 & 640 & 9.1 \\
\hline 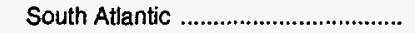 & 601 & 746 & 616 & 614 & 444 & 558 & 578 & 547 & 669 & 592 & 12.5 \\
\hline 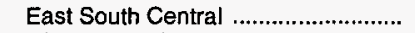 & 571 & 722 & $Q$ & Q & 618 & 501 & 529 & $Q$ & Q & $Q$ & 14.9 \\
\hline 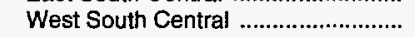 & 612 & 712 & 711 & 664 & 488 & 549 & 556 & $Q$ & $Q$ & $Q$ & 20.1 \\
\hline West & 568 & $Q$ & 629 & 595 & 580 & 520 & 461 & 545 & 757 & 622 & 11.4 \\
\hline 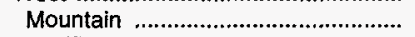 & 599 & $\bar{Q}$ & $Q$ & $\mathbf{Q}$ & 359 & 497 & 507 & $\mathbf{Q}$ & Q & 578 & 14.5 \\
\hline 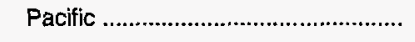 & 555 & $\mathbf{Q}$ & 603 & 550 & 683 & 526 & 441 & $\vec{Q}$ & $\mathrm{Q}$ & 656 & 14.9 \\
\hline \multicolumn{12}{|l|}{ Largest Populated States } \\
\hline 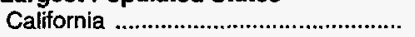 & 559 & $Q$ & $Q$ & $\mathbf{Q}$ & 677 & 547 & 431 & $Q$ & $Q$ & $Q$ & 17.1 \\
\hline Florida & 580 & 0 & $Q$ & $\mathbf{Q}$ & 614 & 541 & $Q$ & Q & $Q$ & $\mathbf{Q}$ & 18.5 \\
\hline 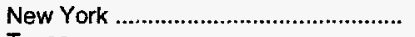 & 545 & $\mathbf{Q}$ & $Q$ & $\mathbf{Q}$ & 761 & 511 & 478 & Q & $Q$ & $\mathbf{Q}$ & 15.1 \\
\hline Texas & 598 & $\mathbf{Q}$ & Q & $\mathbf{Q}$ & 466 & 548 & 563 & $\bar{Q}$ & $\mathrm{Q}$ & $\vec{Q}$ & 26.3 \\
\hline \multicolumn{12}{|l|}{ Urban Status } \\
\hline 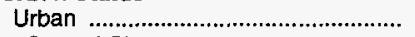 & 565 & 684 & 613 & 594 & 534 & 513 & 510 & 505 & 676 & 662 & 6.1 \\
\hline 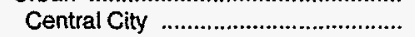 & 535 & 634 & 565 & 571 & 488 & 477 & 478 & 508 & 682 & 615 & 11.9 \\
\hline 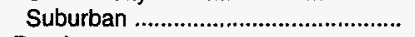 & 581 & 701 & 633 & 606 & 557 & 531 & 528 & 504 & 672 & 685 & 6.7 \\
\hline 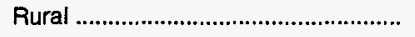 & 617 & 753 & 769 & 663 & 567 & 555 & 547 & 522 & 662 & 710 & 9.5 \\
\hline \multicolumn{12}{|l|}{ Household Size } \\
\hline 1 Person & 492 & 576 & 537 & 499 & 499 & 482 & 403 & 436 & $Q$ & $Q$ & 13.8 \\
\hline 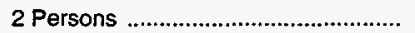 & 553 & 674 & 639 & 577 & 483 & 492 & 505 & 479 & 650 & 612 & 8.1 \\
\hline 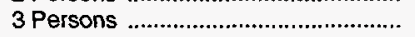 & 596 & 701 & 682 & 628 & 626 & 542 & 519 & 551 & 733 & 665 & 11.1 \\
\hline 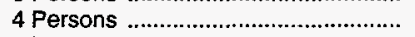 & 615 & 783 & 666 & 675 & 565 & 555 & 584 & 482 & 682 & $Q$ & 11.7 \\
\hline 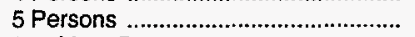 & 630 & $Q$ & 605 & 656 & 533 & 547 & 514 & $Q$ & $\mathrm{Q}$ & $\mathrm{Q}$ & 16.0 \\
\hline 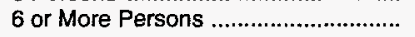 & 673 & $\bar{Q}$ & $\mathrm{Q}$ & Q & $Q$ & Q & $Q$ & $\vec{Q}$ & $\vec{Q}$ & $\vec{Q}$ & 30.1 \\
\hline \multicolumn{12}{|l|}{ Household Composition } \\
\hline $\begin{array}{l}\text { Households with Children } \\
\text { Age of Oldest Child }\end{array}$ & 625 & 761 & 685 & 678 & 607 & 555 & 555 & 546 & 748 & 708 & 7.6 \\
\hline 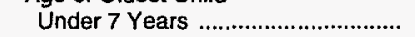 & 606 & $\mathbf{Q}$ & 646 & 620 & 578 & 566 & 536 & $\mathbf{Q}$ & $\mathbf{Q}$ & $\mathbf{Q}$ & 15.3 \\
\hline 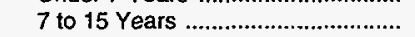 & 633 & 809 & 697 & 713 & 581 & 553 & 559 & 544 & 727 & 699 & 10.6 \\
\hline 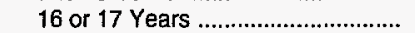 & 630 & $Q$ & 709 & $Q$ & 701 & 549 & 565 & $Q$ & $Q$ & $Q$ & 15.3 \\
\hline Households Without Children ............. & 545 & 650 & 608 & 559 & 501 & 501 & 491 & 479 & 615 & 654 & 6.8 \\
\hline 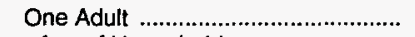 & 492 & 576 & 537 & 499 & 499 & 482 & 403 & 436 & $Q$ & $\mathbf{Q}$ & 13.8 \\
\hline \multicolumn{12}{|l|}{ Age of Householder } \\
\hline Under 35 Years ............................ & 601 & $Q$ & $\mathbf{Q}$ & Q & $Q$ & $\mathbf{Q}$ & Q & Q & $Q$ & $Q$ & 26.6 \\
\hline 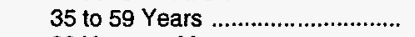 & 547 & $\mathrm{Q}$ & $Q$ & Q & 629 & 538 & $\mathrm{Q}$ & $\vec{Q}$ & $\mathrm{Q}$ & $\overline{\mathbf{Q}}$ & 20.5 \\
\hline 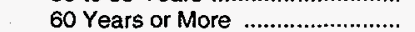 & 384 & $\overrightarrow{\mathrm{Q}}$ & $\bar{Q}$ & $\overline{\mathbf{Q}}$ & 307 & 343 & 348 & $\mathbf{Q}$ & $\vec{Q}$ & $\vec{Q}$ & 18.4 \\
\hline \multirow{2}{*}{\multicolumn{6}{|c|}{ Age of Householder }} & 506 & 513 & 491 & 657 & 641 & 7.5 \\
\hline $\begin{array}{l}\text { Age of Householder } \\
\text { Under } 35 \text { Years }\end{array}$ & 585 & 659 & $\mathbf{Q}$ & $Q$ & 477 & 575 & 543 & $\mathbf{Q}$ & $Q$ & & 18.3 \\
\hline 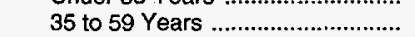 & 594 & 728 & 676 & 583 & 555 & 533 & 541 & 488 & 664 & 741 & 10.8 \\
\hline 60 Years or More .......................... & 495 & 582 & 590 & 507 & 446 & 433 & 459 & 465 & 653 & 508 & 13.3 \\
\hline \multicolumn{12}{|l|}{ Race of Householder } \\
\hline White & 581 & 704 & 644 & 612 & 532 & 526 & 521 & 505 & 669 & 683 & 5.2 \\
\hline Black & 564 & $Q$ & Q & $\mathbf{Q}$ & 618 & 477 & 532 & $\mathrm{Q}$ & Q & $Q$ & 20.2 \\
\hline Other & 531 & $\mathbf{Q}$ & $\mathbf{Q}$ & $\vec{Q}$ & 654 & Q & $\mathbf{Q}$ & $\overline{\mathrm{Q}}$ & $\bar{Q}$ & $\vec{Q}$ & 28.1 \\
\hline
\end{tabular}

See footnotes at end of table. 
Table 5.6. U.S. Average Vehicle Fuel Consumption by Model Year, 1994 (Continued) (Gallons per Vehicle)

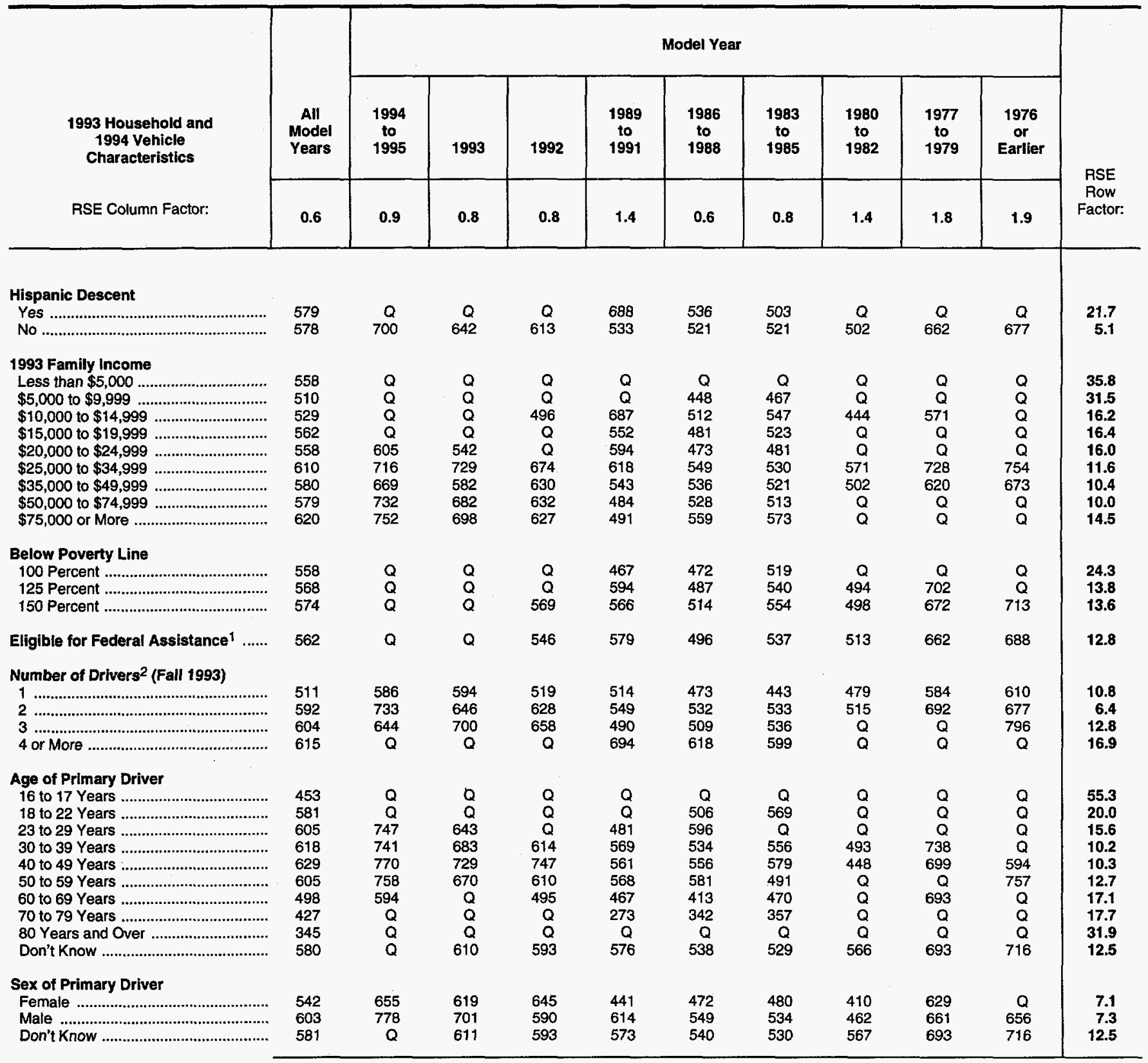

See footnotes at end of table. 
Table 5.6. U.S. Average Vehicle Fuel Consumption by Model Year, 1994 (Continued) (Gallons per Vehicle)

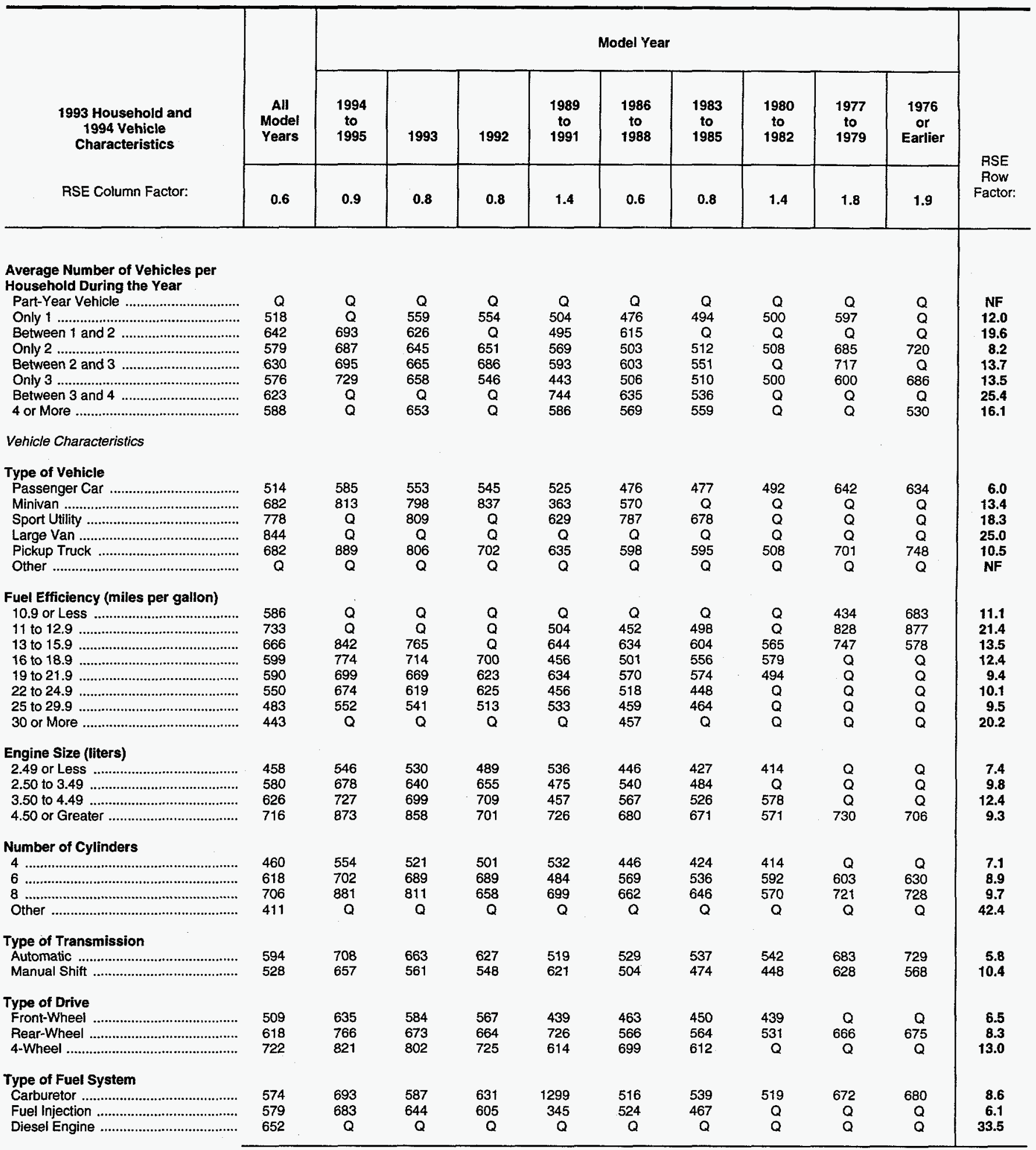

See footnotes at end of table. 
Table 5.6. U.S. Average Vehicle Fuel Consumption by Model Year, 1994 (Continued) (Gallons per Vehicle)

\begin{tabular}{|c|c|c|c|c|c|c|c|c|c|c|c|}
\hline \multirow{3}{*}{$\begin{array}{l}1993 \text { Household and } \\
1994 \text { Vehicle } \\
\text { Characteristics }\end{array}$} & \multirow[b]{2}{*}{$\begin{array}{l}\text { All } \\
\text { Model } \\
\text { Years }\end{array}$} & \multicolumn{9}{|c|}{ Model Year } & \multirow{3}{*}{$\begin{array}{l}\text { RSE } \\
\text { Row } \\
\text { Factor: }\end{array}$} \\
\hline & & $\begin{array}{c}1994 \\
\text { to } \\
1995\end{array}$ & 1993 & 1992 & $\begin{array}{c}1989 \\
\text { to } \\
1991\end{array}$ & $\begin{array}{c}1986 \\
\text { to } \\
1988\end{array}$ & $\begin{array}{l}1983 \\
\text { to } \\
1985\end{array}$ & $\begin{array}{l}1980 \\
\text { to } \\
1982\end{array}$ & $\begin{array}{c}1977 \\
\text { to } \\
1979\end{array}$ & $\begin{array}{c}1976 \\
\text { or } \\
\text { Earlier }\end{array}$ & \\
\hline & 0.6 & 0.9 & 0.8 & 0.8 & 1.4 & 0.6 & 0.8 & 1.4 & 1.8 & 1.9 & \\
\hline \multicolumn{12}{|l|}{ Type of Fuel Purchased } \\
\hline 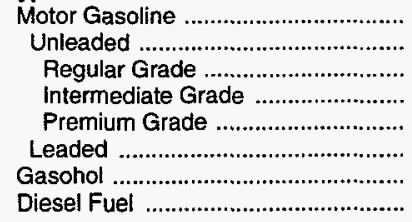 & $\begin{array}{r}575 \\
574 \\
575 \\
567 \\
577 \\
Q \\
663 \\
673\end{array}$ & $\begin{array}{c}674 \\
674 \\
669 \\
624 \\
719 \\
Q \\
Q \\
Q\end{array}$ & $\begin{array}{c}631 \\
631 \\
637 \\
615 \\
625 \\
Q \\
Q \\
Q\end{array}$ & $\begin{array}{r}607 \\
607 \\
619 \\
580 \\
587 \\
Q \\
Q \\
Q\end{array}$ & $\begin{array}{c}541 \\
541 \\
551 \\
431 \\
615 \\
Q \\
Q \\
Q\end{array}$ & $\begin{array}{r}521 \\
521 \\
516 \\
525 \\
539 \\
Q \\
Q \\
Q\end{array}$ & $\begin{array}{r}520 \\
519 \\
518 \\
510 \\
531 \\
Q \\
Q \\
Q\end{array}$ & $\begin{array}{r}517 \\
517 \\
504 \\
Q \\
553 \\
Q \\
Q \\
Q\end{array}$ & $\begin{array}{r}673 \\
667 \\
683 \\
Q \\
647 \\
Q \\
Q \\
Q\end{array}$ & $\begin{array}{c}670 \\
675 \\
693 \\
Q \\
612 \\
Q \\
Q \\
Q\end{array}$ & $\begin{array}{c}5.2 \\
5.2 \\
6.1 \\
12.0 \\
12.8 \\
\text { NF } \\
43.5 \\
37.5\end{array}$ \\
\hline 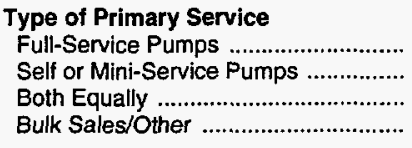 & $\begin{array}{c}492 \\
587 \\
546 \\
Q\end{array}$ & $\begin{array}{r}Q \\
711 \\
Q \\
Q\end{array}$ & $\begin{array}{r}Q \\
646 \\
Q \\
Q\end{array}$ & $\begin{array}{r}\mathrm{Q} \\
617 \\
\mathrm{Q} \\
\mathrm{Q}\end{array}$ & $\begin{array}{r}469 \\
550 \\
Q \\
Q\end{array}$ & $\begin{array}{r}447 \\
530 \\
Q \\
Q\end{array}$ & $\begin{array}{r}421 \\
532 \\
Q \\
Q\end{array}$ & $\begin{array}{r}Q \\
521 \\
Q \\
Q\end{array}$ & $\begin{array}{r}Q \\
682 \\
Q \\
Q\end{array}$ & $\begin{array}{r}Q \\
674 \\
Q \\
Q\end{array}$ & $\begin{array}{r}19.7 \\
5.4 \\
46.7 \\
\text { NF }\end{array}$ \\
\hline \multicolumn{12}{|l|}{$\begin{array}{l}\text { Vehicle Used for Commuting } \\
\text { to and from Work }\end{array}$} \\
\hline Yes & $\begin{array}{l}611 \\
527\end{array}$ & $\begin{array}{l}709 \\
672\end{array}$ & $\begin{array}{l}684 \\
563\end{array}$ & $\begin{array}{l}656 \\
526\end{array}$ & $\begin{array}{l}547 \\
531\end{array}$ & $\begin{array}{l}542 \\
490\end{array}$ & $\begin{array}{l}553 \\
470\end{array}$ & $\begin{array}{l}539 \\
473\end{array}$ & $\begin{array}{l}711 \\
625\end{array}$ & $\begin{array}{l}786 \\
583\end{array}$ & $\begin{array}{l}5.9 \\
8.9\end{array}$ \\
\hline
\end{tabular}

1 Below 150 percent of poverty line or 60 percent of median State income.

2 Approximately 0.5 percent of the vehicle stock was owned by households that had no drivers as of fall 1993.

NF $=$ No applicable RSE row factor.

$Q=$ Data withheld either because the Relative Standard Error (RSE) was greater than 50 percent or fewer than 10 households were sampled.

Notes: "Households with Children" category includes members under age 18 years old unless the member is the householder or spouse. - To obtain the Relative Standard Error (RSE) percentage for any table cell, multiply the corresponding column and row factors. - Because of rounding, data may not sum to totals. - Data in this table are for households with vehicles for personal transportation. - See Glossary for definition of terms used in this report.

Source: Energy Information Administration, Office of Energy Markets and End Use. Form ElA-457 A of the 1993 Residential Energy Consumption Survey and Forms EIA-876 A, B, C, and D of the 1994 Residential Transportation Energy Consumption Survey. 
Table 5.7. U.S. Vehicle-Miles Traveled by Family Income, 1994 (Billion Miles)

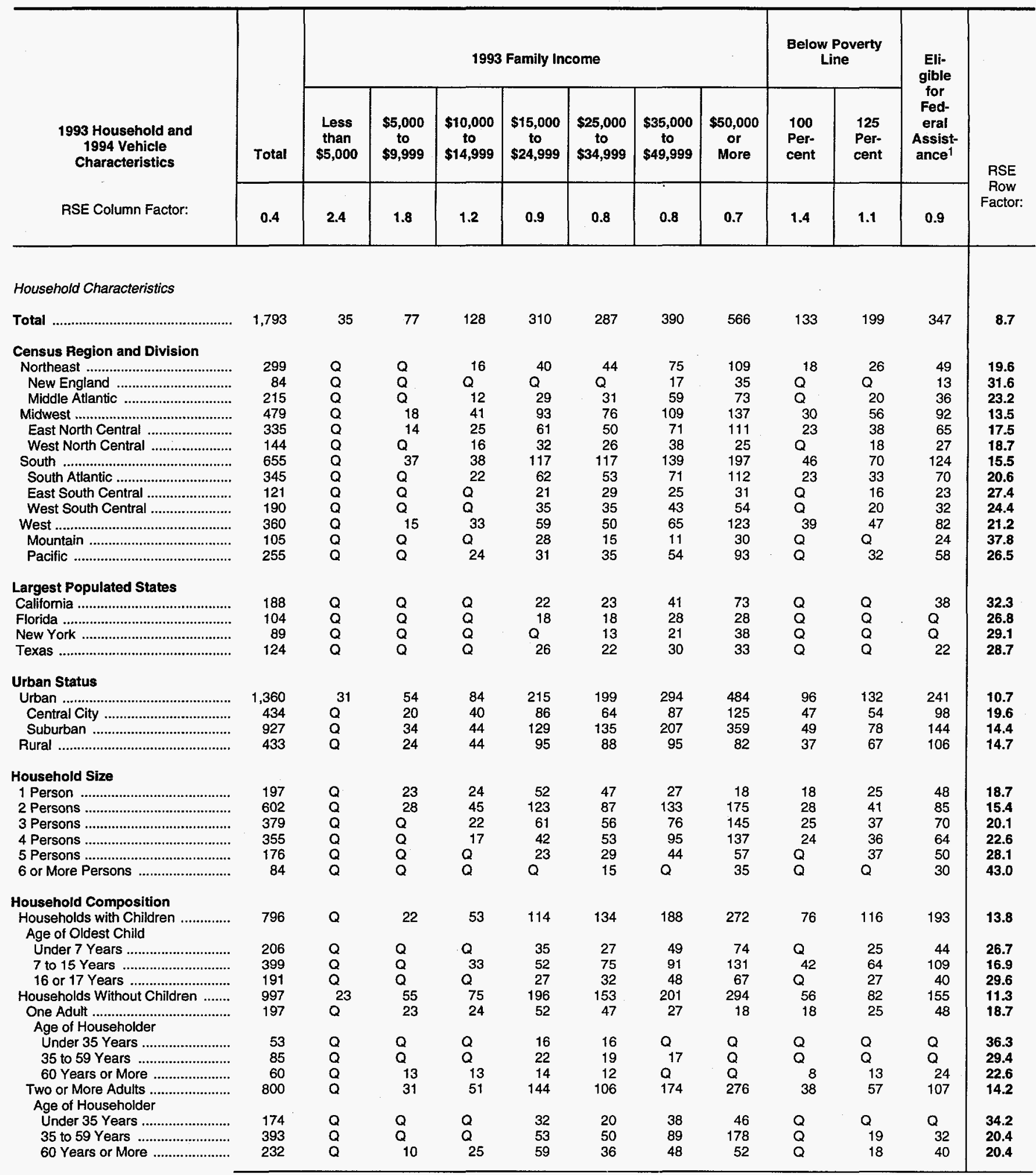

See footnotes at end of table. 
Table 5.7. U.S. Vehicle-Miles Traveled by Family Income, 1994 (Continued) (Billion Miles)

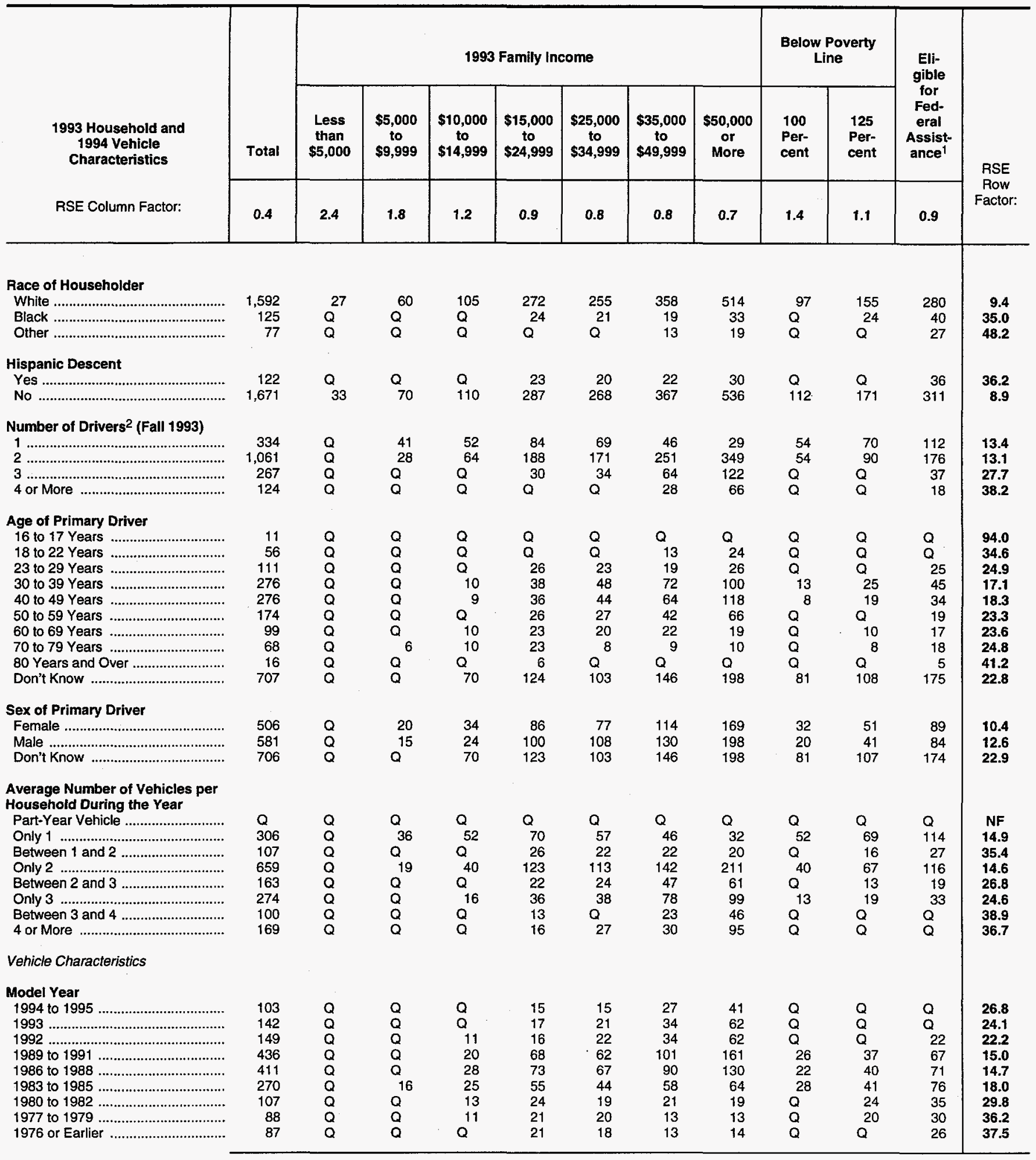

See footnotes at end of table. 
Table 5.7. U.S. Vehicle-Miles Traveled by Family Income, 1994 (Continued) (Billion Miles)

\begin{tabular}{|c|c|c|c|c|c|c|c|c|c|c|c|c|}
\hline \multirow{3}{*}{$\begin{array}{l}1993 \text { Household and } \\
1994 \text { Vehicle } \\
\text { Characteristics }\end{array}$} & \multirow[b]{2}{*}{ Total } & \multicolumn{7}{|c|}{1993 Family Income } & \multicolumn{2}{|c|}{$\begin{array}{c}\text { Below Poverty } \\
\text { Line }\end{array}$} & \multirow{2}{*}{$\begin{array}{l}\text { Eli- } \\
\text { gible } \\
\text { for } \\
\text { Fed- } \\
\text { eral } \\
\text { Assist- } \\
\text { ance }{ }^{1}\end{array}$} & \multirow{3}{*}{$\begin{array}{l}\text { RSE } \\
\text { Row } \\
\text { Factor }\end{array}$} \\
\hline & & $\begin{array}{l}\text { Less } \\
\text { than } \\
\$ 5,000\end{array}$ & $\begin{array}{c}\$ 5,000 \\
\text { to } \\
\$ 9,999\end{array}$ & $\begin{array}{c}\$ 10,000 \\
\text { to } \\
\$ 14,999\end{array}$ & $\begin{array}{c}\$ 15,000 \\
\text { to } \\
\$ 24,999\end{array}$ & $\begin{array}{c}\$ 25,000 \\
\text { to } \\
\$ 34,999\end{array}$ & $\begin{array}{c}\$ 35,000 \\
\text { to } \\
\$ 49,999\end{array}$ & $\begin{array}{c}\$ 50,000 \\
\text { or } \\
\text { More }\end{array}$ & $\begin{array}{l}100 \\
\text { Per- } \\
\text { cent }\end{array}$ & $\begin{array}{l}125 \\
\text { Per- } \\
\text { cent }\end{array}$ & & \\
\hline & 0.4 & 2.4 & 1.8 & 1.2 & 0.9 & 0.8 & 0.8 & 0.7 & 1.4 & 1.1 & 0.9 & \\
\hline 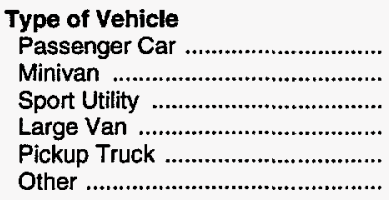 & $\begin{array}{r}1,200 \\
108 \\
121 \\
40 \\
320 \\
Q\end{array}$ & $\begin{array}{l}29 \\
Q \\
Q \\
Q \\
Q \\
Q\end{array}$ & $\begin{array}{l}61 \\
Q \\
Q \\
Q \\
Q \\
Q\end{array}$ & $\begin{array}{l}96 \\
Q \\
Q \\
Q \\
21 \\
Q\end{array}$ & $\begin{array}{r}217 \\
14 \\
16 \\
Q^{57} \\
Q\end{array}$ & $\begin{array}{l}186 \\
19 \\
17 \\
Q \\
59 \\
Q\end{array}$ & $\begin{array}{r}244 \\
29 \\
29 \\
Q \\
78 \\
Q\end{array}$ & $\begin{array}{r}367 \\
40 \\
54 \\
13 \\
90 \\
Q\end{array}$ & $\begin{array}{l}103 \\
Q \\
Q \\
Q \\
Q \\
Q\end{array}$ & $\begin{array}{l}148 \\
Q \\
Q \\
Q \\
Q \\
Q\end{array}$ & $\begin{array}{l}257 \\
Q \\
Q \\
Q^{11} \\
Q^{26}\end{array}$ & $\begin{array}{r}9.9 \\
24.8 \\
27.8 \\
42.6 \\
16.9 \\
\text { NF }\end{array}$ \\
\hline 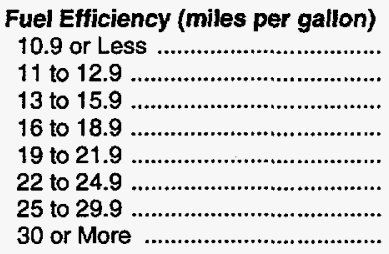 & $\begin{array}{r}39 \\
79 \\
183 \\
278 \\
423 \\
361 \\
324 \\
105\end{array}$ & $\begin{array}{l}Q \\
Q \\
Q \\
Q \\
Q \\
Q \\
Q \\
Q\end{array}$ & $\begin{array}{l}Q \\
Q \\
9 \\
Q^{12} \\
Q \\
Q \\
Q\end{array}$ & $\begin{array}{l}Q^{5} \\
12 \\
18 \\
26 \\
20 \\
28 \\
Q^{28}\end{array}$ & $\begin{array}{r}8 \\
20 \\
31 \\
51 \\
62 \\
56 \\
67 \\
Q\end{array}$ & $\begin{array}{r}6 \\
16 \\
34 \\
39 \\
71 \\
54 \\
51 \\
Q\end{array}$ & $\begin{array}{r}5 \\
15 \\
43 \\
62 \\
92 \\
79 \\
71 \\
22\end{array}$ & $\begin{array}{r}10 \\
15 \\
49 \\
94 \\
153 \\
128 \\
86 \\
31\end{array}$ & $\begin{array}{l}Q \\
Q \\
16 \\
18 \\
24 \\
25 \\
Q \\
Q\end{array}$ & $\begin{array}{l}Q \\
11 \\
22 \\
32 \\
35 \\
36 \\
41 \\
Q\end{array}$ & $\begin{array}{l}13 \\
20 \\
37 \\
51 \\
68 \\
63 \\
73 \\
23\end{array}$ & $\begin{array}{l}34.2 \\
32.5 \\
22.7 \\
15.8 \\
17.9 \\
16.7 \\
15.0 \\
34.3\end{array}$ \\
\hline 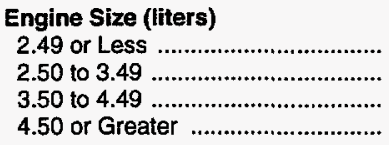 & $\begin{array}{l}699 \\
364 \\
283 \\
448\end{array}$ & $\begin{array}{l}Q \\
Q \\
Q \\
Q\end{array}$ & $\begin{array}{l}Q^{35} \\
Q \\
22\end{array}$ & $\begin{array}{l}54 \\
22 \\
18 \\
34\end{array}$ & $\begin{array}{r}123 \\
51 \\
47 \\
89\end{array}$ & $\begin{array}{r}109 \\
59 \\
43 \\
76\end{array}$ & $\begin{array}{r}145 \\
84 \\
63 \\
97\end{array}$ & $\begin{array}{r}212 \\
129 \\
99 \\
125\end{array}$ & $\begin{array}{l}62 \\
19 \\
18 \\
33\end{array}$ & $\begin{array}{l}89 \\
29 \\
27 \\
54\end{array}$ & $\begin{array}{r}148 \\
59 \\
47 \\
93\end{array}$ & $\begin{array}{l}13.0 \\
15.7 \\
20.6 \\
14.8\end{array}$ \\
\hline Number of Cylinders & $\begin{array}{r}713 \\
631 \\
435 \\
14\end{array}$ & $\begin{array}{l}Q \\
Q \\
Q \\
Q\end{array}$ & $\begin{array}{l}33 \\
20 \\
22 \\
\mathrm{Q}\end{array}$ & $\begin{array}{l}56 \\
40 \\
31 \\
Q\end{array}$ & $\begin{array}{r}124 \\
95 \\
88 \\
a\end{array}$ & $\begin{array}{r}116 \\
99 \\
70 \\
Q\end{array}$ & $\begin{array}{r}149 \\
146 \\
94 \\
Q\end{array}$ & $\begin{array}{l}216 \\
220 \\
124 \\
Q\end{array}$ & $\begin{array}{l}60 \\
38 \\
34 \\
Q\end{array}$ & $\begin{array}{l}88 \\
59 \\
52 \\
Q\end{array}$ & $\begin{array}{c}147 \\
107 \\
91 \\
Q\end{array}$ & $\begin{array}{l}12.0 \\
12.8 \\
15.8 \\
70.9\end{array}$ \\
\hline 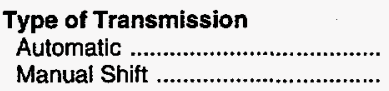 & $\begin{array}{r}1,337 \\
456\end{array}$ & $Q^{24}$ & $Q^{60}$ & $\begin{array}{l}99 \\
29\end{array}$ & $\begin{array}{r}239 \\
71\end{array}$ & $\begin{array}{r}204 \\
83\end{array}$ & $\begin{array}{l}280 \\
110\end{array}$ & $\begin{array}{l}430 \\
136\end{array}$ & $\begin{array}{r}104 \\
29\end{array}$ & $\begin{array}{r}153 \\
45\end{array}$ & $\begin{array}{r}270 \\
77\end{array}$ & $\begin{array}{r}9.2 \\
17.8\end{array}$ \\
\hline 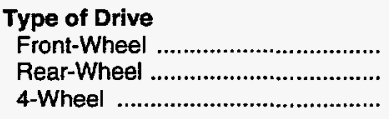 & $\begin{array}{l}891 \\
693 \\
209\end{array}$ & $\mathrm{Q}^{20}$ & $\begin{array}{l}42 \\
30\end{array}$ & $\begin{array}{l}64 \\
51 \\
12\end{array}$ & $\begin{array}{r}145 \\
132 \\
32\end{array}$ & $\begin{array}{r}136 \\
118 \\
33\end{array}$ & $\begin{array}{r}192 \\
146 \\
51\end{array}$ & $\begin{array}{r}290 \\
202 \\
73\end{array}$ & $\begin{array}{l}71 \\
52 \\
Q\end{array}$ & $\begin{array}{r}100 \\
80 \\
18\end{array}$ & $\begin{array}{r}179 \\
141 \\
27\end{array}$ & $\begin{array}{l}10.6 \\
14.8 \\
22.6\end{array}$ \\
\hline 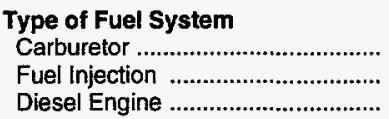 & $\begin{array}{r}802 \\
966 \\
25\end{array}$ & $\begin{array}{l}\mathbf{Q} \\
\mathbf{Q} \\
\mathbf{Q}\end{array}$ & $Q^{49}$ & $\begin{array}{l}74 \\
Q^{53}\end{array}$ & $\begin{array}{l}159 \\
147 \\
Q\end{array}$ & $\begin{array}{l}138 \\
146 \\
Q\end{array}$ & $\begin{array}{l}159 \\
225 \\
Q\end{array}$ & $\begin{array}{l}203 \\
353 \\
Q\end{array}$ & $\begin{array}{l}84 \\
47 \\
Q\end{array}$ & $\begin{array}{r}121 \\
75 \\
Q\end{array}$ & $\begin{array}{l}201 \\
142 \\
Q\end{array}$ & $\begin{array}{l}12.8 \\
10.9 \\
55.1\end{array}$ \\
\hline 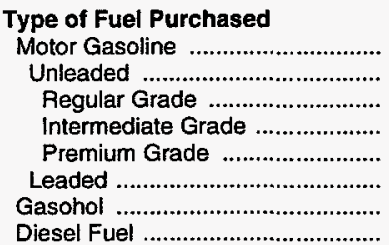 & $\begin{array}{r}1,752 \\
1,736 \\
1,199 \\
238 \\
299 \\
Q \\
17 \\
22\end{array}$ & $\begin{array}{l}34 \\
33 \\
Q \\
Q \\
Q \\
Q \\
Q \\
Q\end{array}$ & $\begin{array}{l}76 \\
75 \\
59 \\
Q \\
Q \\
Q \\
Q \\
Q\end{array}$ & $\begin{array}{r}124 \\
123 \\
89 \\
15 \\
18 \\
Q \\
Q \\
Q\end{array}$ & $\begin{array}{r}302 \\
297 \\
206 \\
35 \\
55 \\
Q \\
Q \\
Q\end{array}$ & $\begin{array}{r}280 \\
277 \\
193 \\
36 \\
48 \\
Q \\
Q \\
Q\end{array}$ & $\begin{array}{r}380 \\
379 \\
252 \\
62 \\
65 \\
Q \\
Q \\
Q\end{array}$ & $\begin{array}{r}555 \\
552 \\
379 \\
79 \\
94 \\
Q \\
Q \\
Q\end{array}$ & $\begin{array}{l}130 \\
127 \\
93 \\
Q \\
21 \\
Q \\
Q \\
Q\end{array}$ & $\begin{array}{l}192 \\
189 \\
135 \\
20 \\
34 \\
Q \\
Q \\
Q\end{array}$ & $\begin{array}{c}339 \\
334 \\
240 \\
37 \\
58 \\
Q \\
Q \\
Q\end{array}$ & $\begin{array}{c}9.0 \\
9.0 \\
10.5 \\
20.5 \\
18.6 \\
\text { NF } \\
70.3 \\
61.1\end{array}$ \\
\hline
\end{tabular}

See footnotes at end of table. 
Table 5.7. U.S. Vehicle-Miles Traveled by Family Income, 1994 (Continued) (Billion Miles)

\begin{tabular}{|c|c|c|c|c|c|c|c|c|c|c|c|c|}
\hline \multirow[b]{2}{*}{$\begin{array}{c}1993 \text { Household and } \\
1994 \text { Vehicle } \\
\text { Characteristics }\end{array}$} & \multirow[b]{2}{*}{ Total } & \multicolumn{7}{|c|}{1993 Family Income } & \multicolumn{2}{|c|}{$\begin{array}{l}\text { Below Poverty } \\
\text { Line }\end{array}$} & \multirow{2}{*}{$\begin{array}{l}\text { Eli- } \\
\text { gible } \\
\text { for } \\
\text { Fed- } \\
\text { eral } \\
\text { Assist-- } \\
\text { ance }\end{array}$} & \multirow{3}{*}{$\begin{array}{l}\text { RSE } \\
\text { Row } \\
\text { Factor: }\end{array}$} \\
\hline & & $\begin{array}{l}\text { Less } \\
\text { than } \\
\$ 5,000\end{array}$ & $\begin{array}{l}\$ 5,000 \\
\text { to } \\
\$ 9,999\end{array}$ & $\begin{array}{c}\$ 10,000 \\
\text { to } \\
\$ 14,999\end{array}$ & $\begin{array}{c}\$ 15,000 \\
\text { to } \\
\$ 24,999\end{array}$ & $\begin{array}{c}\$ 25,000 \\
\text { to } \\
\$ 34,999\end{array}$ & $\begin{array}{c}\$ 35,000 \\
\text { to } \\
\$ 49,999\end{array}$ & $\begin{array}{c}\$ 50,000 \\
\text { or } \\
\text { More }\end{array}$ & $\begin{array}{l}100 \\
\text { Per- } \\
\text { cent }\end{array}$ & $\begin{array}{l}125 \\
\text { Per- } \\
\text { cent }\end{array}$ & & \\
\hline RSE Column Factor: & 0.4 & 2.4 & 1.8 & 1.2 & 0.9 & 0.8 & 0.8 & 0.7 & 1.4 & 1.1 & 0.9 & \\
\hline $\begin{array}{l}\text { Type of Primary Service } \\
\text { Full-Service Pumps ...................... } \\
\text { Self or Mini-Service Pumps .......... } \\
\text { Both Equally .................................. } \\
\text { Bulk Sales/Other ......................... }\end{array}$ & $\begin{array}{r}137 \\
1,624 \\
30 \\
Q\end{array}$ & $\begin{array}{l}Q \\
31 \\
Q \\
Q\end{array}$ & $\begin{array}{l}Q \\
Q 6 \\
Q \\
Q\end{array}$ & $\begin{array}{l}11 \\
114 \\
Q \\
Q\end{array}$ & $\begin{array}{l}18 \\
285 \\
Q \\
Q\end{array}$ & $\begin{array}{l}20 \\
263 \\
Q \\
Q\end{array}$ & $\begin{array}{l}33 \\
349 \\
Q \\
Q\end{array}$ & $\begin{array}{l}41 \\
516 \\
Q \\
Q\end{array}$ & $\begin{array}{l}Q \\
116 \\
Q \\
Q\end{array}$ & $\begin{array}{l}18 \\
179 \\
Q \\
Q\end{array}$ & $\begin{array}{l}33 \\
308 \\
Q \\
Q\end{array}$ & $\begin{array}{r}33.2 \\
9.4 \\
77.9 \\
\text { NF }\end{array}$ \\
\hline $\begin{array}{l}\text { Vehicle Used for Commuting } \\
\text { to and from Work } \\
\text { Yes } \\
\text { No }\end{array}$ & $\begin{array}{r}1,175 \\
618\end{array}$ & Q & $\begin{array}{l}39 \\
38\end{array}$ & $\begin{array}{l}67 \\
61\end{array}$ & $\begin{array}{l}187 \\
123\end{array}$ & $\begin{array}{l}187 \\
101\end{array}$ & $\begin{array}{l}258 \\
132\end{array}$ & $\begin{array}{l}420 \\
146\end{array}$ & $\begin{array}{l}74 \\
59\end{array}$ & $\begin{array}{r}109 \\
89\end{array}$ & $\begin{array}{l}199 \\
148\end{array}$ & $\begin{array}{l}10.7 \\
12.3\end{array}$ \\
\hline
\end{tabular}

1 Below 150 percent of poverty line or 60 percent of median State income.

2 Approximately 0.5 percent of the vehicle stock was owned by households that had no drivers as of fall 1993.

NF = No applicable RSE row factor.

$Q=$ Data withheld either because the Relative Standard Error (RSE) was greater than 50 percent or fewer than 10 households were sampled.

Notes: "Households with Children" category includes miembers under age 18 years old unless the member is the householder or spouse. $\bullet$ To obtain the Relative Standard Error (RSE) percentage for any table cell, multiply the corresponding column and row factors. - Because of rounding, data may not sum to totals. • Data in this table are for households with vehicles for personal transportation. - See Glossary for definition of terms used in this report.

Source: Energy Information Administration, Office of Energy Markets and End Use, Form EIA-457 A of the 1993 Residential Energy Consumption Survey and Forms EIA-876 A, B, C, and D of the 1994 Residential Transportation Energy Consumption Survey. 
Table 5.8. U.S. Vehicle Fuel Consumption by Family Income, 1994 (Billion Gallons)

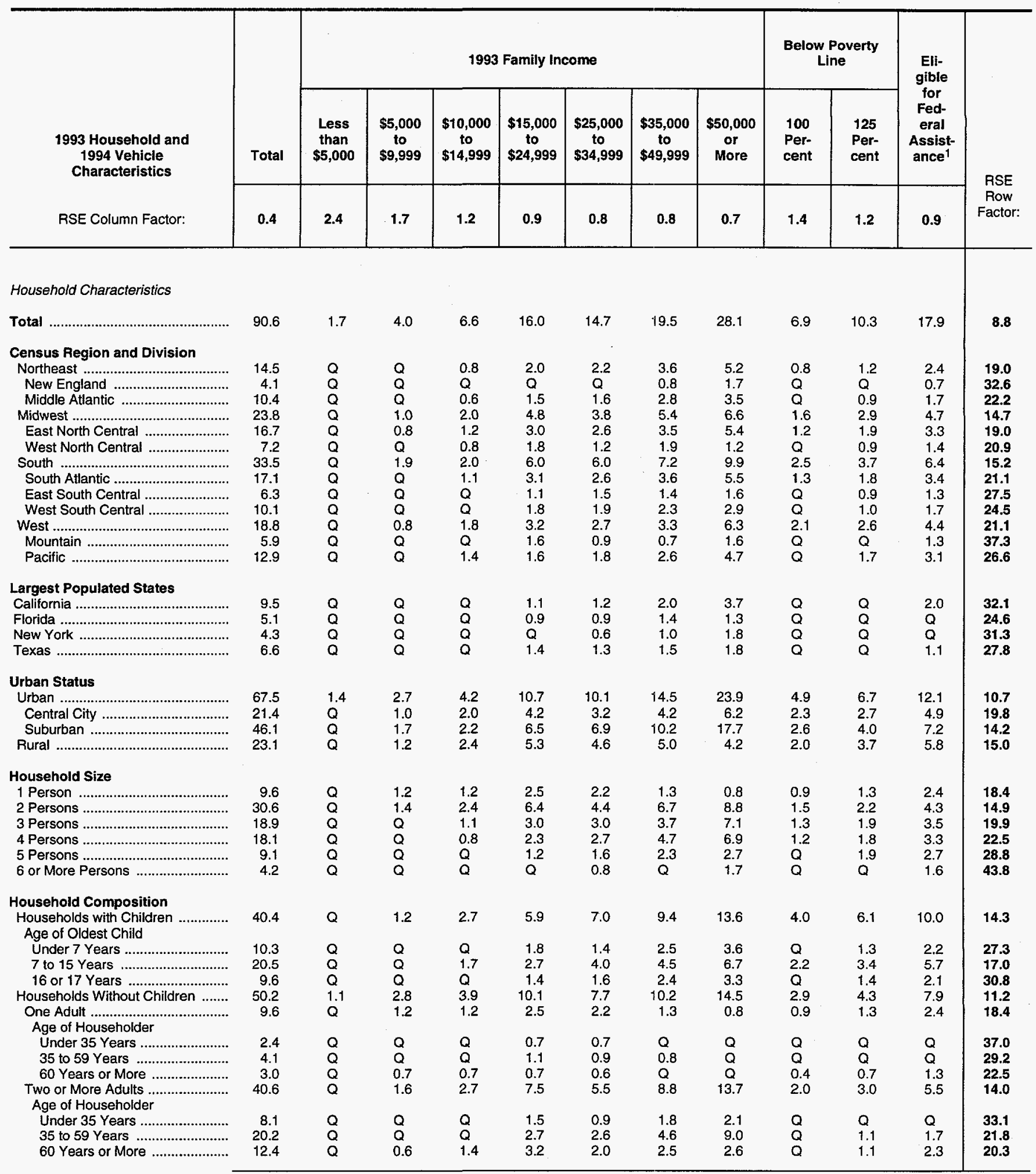

See footnotes at end of table. 
Table 5.8. U.S. Vehicle Fuel Consumption by Family Income, 1994 (Continued) (Billion Gallons)

\begin{tabular}{|c|c|c|c|c|c|c|c|c|c|c|c|c|}
\hline \multirow{3}{*}{$\begin{array}{l}1993 \text { Household and } \\
1994 \text { Vehicle } \\
\text { Characteristics }\end{array}$} & \multirow[b]{2}{*}{ Total } & \multicolumn{7}{|c|}{1993 Family Income } & \multicolumn{2}{|c|}{$\begin{array}{c}\text { Below Poverty } \\
\text { Line }\end{array}$} & \multirow{2}{*}{$\begin{array}{l}\text { Eli- } \\
\text { gible } \\
\text { for } \\
\text { Fed- } \\
\text { eral } \\
\text { Assist- } \\
\text { ance }{ }^{1}\end{array}$} & \multirow{3}{*}{$\begin{array}{l}\text { RSE } \\
\text { Row } \\
\text { Factor: }\end{array}$} \\
\hline & & $\begin{array}{l}\text { Less } \\
\text { than } \\
\$ 5,000\end{array}$ & $\begin{array}{l}\$ 5,000 \\
\text { to } \\
\$ 9,999\end{array}$ & $\begin{array}{c}\$ 10,000 \\
\text { to } \\
\$ 14,999\end{array}$ & $\begin{array}{c}\$ 15,000 \\
\text { to } \\
\$ 24,999\end{array}$ & $\begin{array}{l}\$ 25,000 \\
\text { to } \\
\$ 34,999\end{array}$ & $\begin{array}{c}\$ 35,000 \\
\text { to } \\
\$ 49,999\end{array}$ & $\begin{array}{l}\mathbf{5 0 , 0 0 0} \\
\text { or } \\
\text { More }\end{array}$ & $\begin{array}{l}100 \\
\text { Per- } \\
\text { cent }\end{array}$ & $\begin{array}{l}125 \\
\text { Per- } \\
\text { cent }\end{array}$ & & \\
\hline & 0.4 & 2.4 & 1.7 & 1.2 & 0.9 & 0.8 & 0.8 & 0.7 & 1.4 & 1.2 & 0.9 & \\
\hline \multicolumn{13}{|l|}{ Race of Householder } \\
\hline 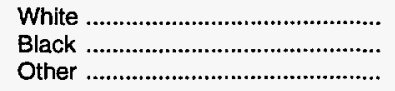 & $\begin{array}{r}80.5 \\
6.3 \\
3.7\end{array}$ & $\begin{array}{l}1.3 \\
Q \\
Q\end{array}$ & $\begin{array}{l}3.0 \\
Q \\
Q\end{array}$ & $\begin{array}{l}5.4 \\
Q \\
Q\end{array}$ & $\begin{array}{c}14.1 \\
1.2 \\
Q\end{array}$ & $\begin{array}{c}13.2 \\
0.9 \\
Q\end{array}$ & $\begin{array}{r}17.9 \\
1.0 \\
0.6\end{array}$ & $\begin{array}{r}25.6 \\
1.6 \\
0.9\end{array}$ & $\begin{array}{l}5.0 \\
Q \\
Q\end{array}$ & $\begin{array}{l}8.0 \\
1.4 \\
Q\end{array}$ & $\begin{array}{r}14.3 \\
2.2 \\
1.4\end{array}$ & $\begin{array}{r}9.5 \\
34.9 \\
48.7\end{array}$ \\
\hline \multicolumn{13}{|l|}{ Hispanic Descent } \\
\hline Yes No & $\begin{array}{r}6.2 \\
84.4\end{array}$ & $\begin{array}{l}Q \\
1.6\end{array}$ & $\begin{array}{l}Q \\
3.6\end{array}$ & $\begin{array}{l}Q \\
5.7\end{array}$ & $\begin{array}{r}1.1 \\
14.9\end{array}$ & $\begin{array}{r}1.1 \\
13.6\end{array}$ & $\begin{array}{r}1.1 \\
18.4\end{array}$ & $\begin{array}{r}1.5 \\
26.6\end{array}$ & $\begin{array}{l}Q \\
5.9\end{array}$ & $\begin{array}{l}Q \\
8.9\end{array}$ & $\begin{array}{r}1.8 \\
16.1\end{array}$ & $\begin{array}{r}37.2 \\
9.0\end{array}$ \\
\hline \multicolumn{13}{|l|}{ Number of Drivers ${ }^{2}$ (Fall 1993) } \\
\hline 3 or More & $\begin{array}{r}16.5 \\
54.3 \\
13.4 \\
6.0\end{array}$ & $\begin{array}{l}Q \\
Q \\
Q \\
Q\end{array}$ & $\begin{array}{l}2.1 \\
1.4 \\
\mathbf{Q} \\
\mathbf{Q}\end{array}$ & $\begin{array}{l}2.6 \\
3.3 \\
Q \\
Q\end{array}$ & $\begin{array}{l}4.2 \\
9.9 \\
1.5 \\
Q\end{array}$ & $\begin{array}{l}3.2 \\
8.9 \\
1.9 \\
Q\end{array}$ & $\begin{array}{r}2.2 \\
12.7 \\
3.2 \\
1.3\end{array}$ & $\begin{array}{r}1.5 \\
17.6 \\
5.9 \\
3.2\end{array}$ & $\begin{array}{l}2.7 \\
2.9 \\
Q \\
Q\end{array}$ & $\begin{array}{l}3.4 \\
4.9 \\
Q \\
Q\end{array}$ & $\begin{array}{l}5.6 \\
9.1 \\
2.0 \\
0.8\end{array}$ & $\begin{array}{l}13.9 \\
12.8 \\
27.5 \\
39.6\end{array}$ \\
\hline $\begin{array}{l}\text { Age of Primary Driver } \\
16 \text { to } 17 \text { Years } \\
18 \text { to } 22 \text { Years } \\
23 \text { to } 29 \text { Years } \\
30 \text { to } 39 \text { Years } \\
40 \text { to } 49 \text { Years } \\
50 \text { to } 59 \text { Years } \\
60 \text { to } 69 \text { Years } \\
70 \text { to } 79 \text { Years } \\
80 \text { Years and Over } \\
\text { Don't Know }\end{array}$ & $\begin{array}{r}0.5 \\
2.5 \\
5.1 \\
14.0 \\
14.0 \\
9.0 \\
5.3 \\
3.7 \\
0.9 \\
35.6\end{array}$ & $\begin{array}{l}Q \\
Q \\
Q \\
Q \\
Q \\
Q \\
Q \\
Q \\
Q \\
Q\end{array}$ & $\begin{array}{l}Q \\
Q \\
Q \\
Q \\
Q \\
Q \\
Q \\
0.3 \\
Q \\
Q\end{array}$ & $\begin{array}{l}Q \\
Q \\
Q \\
0.6 \\
0.4 \\
Q \\
0.5 \\
0.6 \\
Q \\
3.6\end{array}$ & $\begin{array}{l}Q \\
Q \\
1.2 \\
2.0 \\
1.8 \\
1.4 \\
1.2 \\
1.2 \\
0.4 \\
6.4\end{array}$ & $\begin{array}{l}Q \\
Q \\
1.0 \\
2.6 \\
2.2 \\
1.5 \\
1.2 \\
0.4 \\
Q \\
5.1\end{array}$ & $\begin{array}{l}Q \\
0.6 \\
0.9 \\
3.6 \\
3.2 \\
2.1 \\
1.1 \\
0.4 \\
Q \\
7.3\end{array}$ & $\begin{array}{l}Q \\
1.0 \\
1.2 \\
4.9 \\
6.1 \\
3.4 \\
0.9 \\
0.5 \\
Q \\
9.9\end{array}$ & $\begin{array}{l}Q \\
Q \\
Q \\
0.7 \\
0.4 \\
Q \\
Q \\
Q \\
Q \\
4.1\end{array}$ & $\begin{array}{l}Q \\
Q \\
Q \\
1.3 \\
0.9 \\
Q \\
0.6 \\
0.5 \\
Q \\
5.5\end{array}$ & $\begin{array}{l}Q \\
Q \\
1.1 \\
2.4 \\
1.7 \\
1.0 \\
1.0 \\
1.0 \\
0.3 \\
8.9\end{array}$ & $\begin{array}{l}93.8 \\
35.7 \\
25.5 \\
17.5 \\
18.2 \\
23.9 \\
23.8 \\
24.6 \\
40.1 \\
22.5\end{array}$ \\
\hline 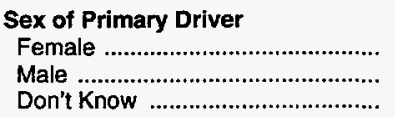 & $\begin{array}{l}23.5 \\
31.5 \\
35.6\end{array}$ & $\begin{array}{l}Q \\
Q \\
Q\end{array}$ & $\begin{array}{l}1.0 \\
0.9 \\
Q\end{array}$ & $\begin{array}{l}1.6 \\
1.4 \\
3.6\end{array}$ & $\begin{array}{l}3.9 \\
5.7 \\
6.4\end{array}$ & $\begin{array}{l}3.7 \\
5.9 \\
5.1\end{array}$ & $\begin{array}{l}5.2 \\
7.0 \\
7.3\end{array}$ & $\begin{array}{r}7.9 \\
10.2 \\
9.9\end{array}$ & $\begin{array}{l}1.6 \\
1.3 \\
4.1\end{array}$ & $\begin{array}{l}2.4 \\
2.4 \\
5.5\end{array}$ & $\begin{array}{l}4.1 \\
4.9 \\
8.9\end{array}$ & $\begin{array}{l}10.5 \\
13.3 \\
22.6\end{array}$ \\
\hline $\begin{array}{l}\text { Average Number of Vehicles per } \\
\text { Household During the Year } \\
\text { Part-Year Vehicle ..................... } \\
\text { Only } 1 \\
\text { Between } 1 \text { and } 2 \\
\text { Only } 2 \\
\text { Between } 2 \text { and } 3 \\
\text { Only } 3 \\
\text { Between } 3 \text { and } 4 \\
4 \text { or More }\end{array}$ & \begin{tabular}{r}
\multicolumn{1}{c}{$Q$} \\
14.8 \\
5.4 \\
32.9 \\
8.5 \\
14.5 \\
4.9 \\
8.7
\end{tabular} & $\begin{array}{l}Q \\
Q \\
Q \\
Q \\
Q \\
Q \\
Q \\
Q\end{array}$ & $\begin{array}{l}Q \\
1.9 \\
Q \\
1.1 \\
Q \\
Q \\
Q \\
Q\end{array}$ & $\begin{array}{l}Q \\
2.5 \\
Q \\
2.1 \\
Q \\
1.0 \\
Q \\
Q\end{array}$ & $\begin{array}{l}Q \\
3.4 \\
1.3 \\
6.4 \\
1.2 \\
2.0 \\
0.6 \\
0.9\end{array}$ & $\begin{array}{l}Q \\
2.6 \\
1.1 \\
5.8 \\
1.2 \\
2.1 \\
Q \\
1.4\end{array}$ & $\begin{array}{l}Q \\
2.2 \\
1.1 \\
6.9 \\
2.5 \\
4.1 \\
1.2 \\
1.5\end{array}$ & $\begin{array}{r}Q \\
1.6 \\
1.0 \\
10.3 \\
3.1 \\
5.0 \\
2.4 \\
4.8\end{array}$ & $\begin{array}{l}Q \\
2.6 \\
Q \\
2.1 \\
Q \\
0.8 \\
Q \\
Q\end{array}$ & $\begin{array}{l}Q \\
3.4 \\
0.8 \\
3.6 \\
0.8 \\
1.1 \\
Q \\
Q\end{array}$ & $\begin{array}{l}Q \\
5.7 \\
1.4 \\
6.2 \\
1.1 \\
1.8 \\
Q \\
Q\end{array}$ & $\begin{array}{c}\text { NF } \\
15.0 \\
35.2 \\
15.0 \\
27.0 \\
24.8 \\
40.0 \\
36.2\end{array}$ \\
\hline \multicolumn{13}{|l|}{ Vehicle Characteristics } \\
\hline \multicolumn{13}{|l|}{ Model Year } \\
\hline 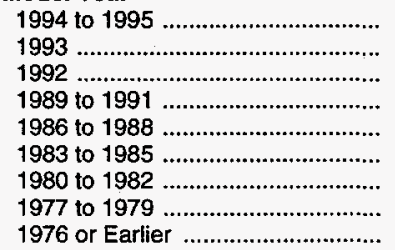 & $\begin{array}{r}5.0 \\
6.6 \\
6.9 \\
20.7 \\
19.0 \\
13.3 \\
5.6 \\
6.3 \\
7.2\end{array}$ & $\begin{array}{l}Q \\
Q \\
Q \\
Q \\
Q \\
Q \\
Q \\
Q \\
Q\end{array}$ & $\begin{array}{l}Q \\
Q \\
Q \\
Q \\
0.8 \\
0.8 \\
Q \\
Q \\
Q\end{array}$ & $\begin{array}{l}Q \\
Q \\
0.5 \\
0.9 \\
1.3 \\
1.2 \\
0.6 \\
0.8 \\
Q\end{array}$ & $\begin{array}{l}0.7 \\
0.7 \\
0.7 \\
3.2 \\
3.3 \\
2.8 \\
1.2 \\
1.5 \\
1.8\end{array}$ & $\begin{array}{l}0.7 \\
1.0 \\
1.0 \\
2.9 \\
3.1 \\
2.2 \\
1.0 \\
1.4 \\
1.5\end{array}$ & $\begin{array}{l}1.3 \\
1.5 \\
1.6 \\
4.9 \\
4.3 \\
2.9 \\
1.1 \\
0.9 \\
1.0\end{array}$ & $\begin{array}{l}2.1 \\
3.0 \\
2.9 \\
7.6 \\
6.1 \\
3.2 \\
1.0 \\
1.0 \\
1.2\end{array}$ & $\begin{array}{l}Q \\
Q \\
Q \\
1.2 \\
1.0 \\
1.2 \\
Q \\
Q \\
Q\end{array}$ & $\begin{array}{l}Q \\
Q \\
Q \\
1.7 \\
1.8 \\
1.9 \\
1.2 \\
1.4 \\
Q\end{array}$ & $\begin{array}{l}Q \\
Q \\
0.9 \\
3.0 \\
3.2 \\
3.6 \\
1.8 \\
2.2 \\
2.2\end{array}$ & $\begin{array}{l}26.6 \\
23.7 \\
22.4 \\
15.1 \\
15.3 \\
17.8 \\
28.3 \\
34.6 \\
36.8\end{array}$ \\
\hline
\end{tabular}

See footnotes at end of table. 
Table 5.8. U.S. Vehicle Fuel Consumption by Family Income, 1994 (Continued) (Billion Gallons)

\begin{tabular}{|c|c|c|c|c|c|c|c|c|c|c|c|c|}
\hline \multirow{3}{*}{$\begin{array}{l}1993 \text { Household and } \\
1994 \text { Vehicle } \\
\text { Characteristics }\end{array}$} & \multirow[b]{2}{*}{ Total } & \multicolumn{7}{|c|}{1993 Family Income } & \multicolumn{2}{|c|}{$\begin{array}{c}\text { Below Poverty } \\
\text { Line }\end{array}$} & \multirow{2}{*}{$\begin{array}{l}\text { Eli- } \\
\text { gible } \\
\text { for } \\
\text { Fed- } \\
\text { eral } \\
\text { Assist- } \\
\text { ance }\end{array}$} & \multirow{3}{*}{$\begin{array}{l}\text { RSE } \\
\text { Row } \\
\text { Factor: }\end{array}$} \\
\hline & & $\begin{array}{l}\text { Less } \\
\text { than } \\
\$ 5,000\end{array}$ & $\begin{array}{l}\$ 5,000 \\
\text { to } \\
\$ 9,999\end{array}$ & $\begin{array}{c}\$ 10,000 \\
\text { to } \\
\$ 14,999\end{array}$ & $\begin{array}{c}\$ 15,000 \\
\text { to } \\
\$ 24,999\end{array}$ & $\begin{array}{c}\$ 25,000 \\
\text { to } \\
\$ 34,999\end{array}$ & $\begin{array}{c}\$ 35,000 \\
\text { to } \\
\$ 49,999\end{array}$ & $\begin{array}{c}\$ 50,000 \\
\text { or } \\
\text { More }\end{array}$ & $\begin{array}{l}100 \\
\text { Per- } \\
\text { cent }\end{array}$ & $\begin{array}{l}125 \\
\text { Per- } \\
\text { cent }\end{array}$ & & \\
\hline & 0.4 & 2.4 & 1.7 & 1.2 & 0.9 & 0.8 & 0.8 & 0.7 & 1.4 & 1.2 & 0.9 & \\
\hline 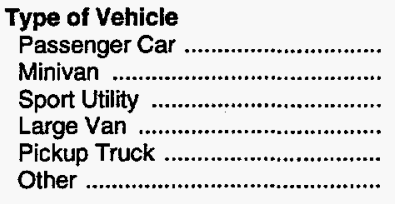 & $\begin{array}{r}54.7 \\
5.5 \\
7.4 \\
2.9 \\
19.6 \\
Q\end{array}$ & $\begin{array}{l}1.3 \\
Q \\
Q \\
Q \\
Q \\
Q\end{array}$ & $\begin{array}{l}2.9 \\
Q \\
Q \\
Q \\
Q \\
Q\end{array}$ & $\begin{array}{l}4.6 \\
Q \\
Q \\
Q \\
1.3 \\
Q\end{array}$ & $\begin{array}{l}10.1 \\
0.7 \\
1.0 \\
Q \\
3.6 \\
Q\end{array}$ & $\begin{array}{l}8.6 \\
0.9 \\
1.1 \\
Q \\
3.6 \\
Q\end{array}$ & $\begin{array}{l}10.8 \\
1.5 \\
1.8 \\
Q \\
4.8 \\
Q\end{array}$ & $\begin{array}{l}16.5 \\
2.0 \\
3.2 \\
0.9 \\
5.3 \\
Q\end{array}$ & $\begin{array}{l}4.9 \\
Q \\
Q \\
Q \\
Q \\
Q\end{array}$ & $\begin{array}{l}7.0 \\
Q \\
Q \\
Q \\
2.0 \\
Q\end{array}$ & $\begin{array}{l}12.1 \\
Q \\
0.7 \\
Q \\
3.6 \\
Q\end{array}$ & $\begin{array}{r}9.9 \\
24.7 \\
27.5 \\
42.2 \\
17.1 \\
\text { NF }\end{array}$ \\
\hline 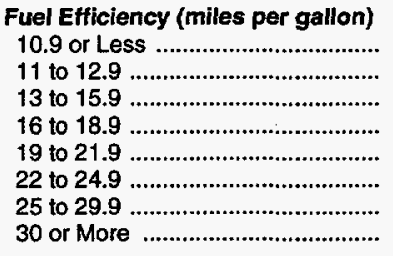 & $\begin{array}{r}4.1 \\
6.5 \\
12.6 \\
15.9 \\
20.8 \\
15.5 \\
12.0 \\
3.2\end{array}$ & $\begin{array}{l}Q \\
Q \\
Q \\
Q \\
Q \\
Q \\
Q \\
Q\end{array}$ & $\begin{array}{l}Q \\
Q \\
0.6 \\
0.7 \\
Q \\
Q \\
Q \\
Q\end{array}$ & $\begin{array}{l}0.5 \\
Q \\
0.8 \\
1.0 \\
1.3 \\
0.9 \\
1.1 \\
Q\end{array}$ & $\begin{array}{l}0.9 \\
1.6 \\
2.1 \\
2.9 \\
3.1 \\
2.4 \\
2.5 \\
Q\end{array}$ & $\begin{array}{l}0.6 \\
1.3 \\
2.3 \\
2.3 \\
3.5 \\
2.3 \\
1.9 \\
Q\end{array}$ & $\begin{array}{l}0.6 \\
1.2 \\
3.0 \\
3.5 \\
4.5 \\
3.4 \\
2.6 \\
0.7\end{array}$ & $\begin{array}{l}1.1 \\
1.2 \\
3.4 \\
5.3 \\
7.5 \\
5.5 \\
3.2 \\
0.9\end{array}$ & $\begin{array}{l}Q \\
Q \\
1.1 \\
1.0 \\
1.2 \\
1.1 \\
Q \\
Q\end{array}$ & $\begin{array}{l}Q \\
1.0 \\
1.5 \\
1.8 \\
1.7 \\
1.5 \\
1.5 \\
Q\end{array}$ & $\begin{array}{l}1.4 \\
1.7 \\
2.5 \\
2.9 \\
3.3 \\
2.7 \\
2.7 \\
0.7\end{array}$ & $\begin{array}{l}33.8 \\
32.3 \\
22.6 \\
15.8 \\
17.8 \\
16.6 \\
14.8 \\
34.9\end{array}$ \\
\hline 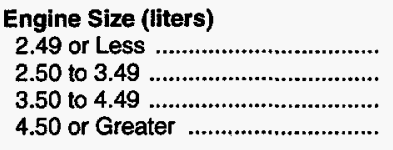 & $\begin{array}{l}27.4 \\
17.5 \\
15.1 \\
30.6\end{array}$ & $\begin{array}{l}Q \\
Q \\
Q \\
Q\end{array}$ & $\begin{array}{l}1.4 \\
Q \\
Q \\
1.6\end{array}$ & $\begin{array}{l}2.1 \\
1.1 \\
1.0 \\
2.4\end{array}$ & $\begin{array}{l}4.8 \\
2.4 \\
2.6 \\
6.2\end{array}$ & $\begin{array}{l}4.3 \\
2.9 \\
2.3 \\
5.3\end{array}$ & $\begin{array}{l}5.7 \\
4.0 \\
3.3 \\
6.5\end{array}$ & $\begin{array}{l}8.5 \\
6.2 \\
5.2 \\
8.2\end{array}$ & $\begin{array}{l}2.5 \\
0.9 \\
1.0 \\
2.5\end{array}$ & $\begin{array}{l}3.5 \\
1.4 \\
1.5 \\
3.9\end{array}$ & $\begin{array}{l}5.7 \\
2.9 \\
2.6 \\
6.7\end{array}$ & $\begin{array}{l}12.5 \\
15.3 \\
20.2 \\
14.7\end{array}$ \\
\hline Number of Cylinders & $\begin{array}{r}28.3 \\
32.3 \\
29.4 \\
0.5\end{array}$ & $\begin{array}{l}Q \\
Q \\
Q \\
Q\end{array}$ & $\begin{array}{l}1.4 \\
1.1 \\
1.5 \\
Q\end{array}$ & $\begin{array}{l}2.2 \\
2.1 \\
2.3 \\
Q\end{array}$ & $\begin{array}{l}4.9 \\
4.9 \\
6.1 \\
Q\end{array}$ & $\begin{array}{l}4.6 \\
5.2 \\
4.8 \\
Q\end{array}$ & $\begin{array}{l}5.9 \\
7.3 \\
6.3 \\
Q\end{array}$ & $\begin{array}{r}8.7 \\
11.2 \\
8.0 \\
Q\end{array}$ & $\begin{array}{l}2.4 \\
2.0 \\
2.5 \\
Q\end{array}$ & $\begin{array}{l}3.5 \\
3.1 \\
3.7 \\
Q\end{array}$ & $\begin{array}{l}5.8 \\
5.6 \\
6.4 \\
Q\end{array}$ & $\begin{array}{l}11.5 \\
12.6 \\
15.6 \\
71.9\end{array}$ \\
\hline $\begin{array}{l}\text { Type of Transmission } \\
\text { Automatic ....................................... } \\
\text { Manual Shift ................................ }\end{array}$ & $\begin{array}{l}69.8 \\
20.7\end{array}$ & $\begin{array}{l}1.3 \\
Q\end{array}$ & $\begin{array}{l}3.3 \\
Q\end{array}$ & $\begin{array}{l}5.3 \\
1.3\end{array}$ & $\begin{array}{r}12.6 \\
3.4\end{array}$ & $\begin{array}{r}10.9 \\
3.8\end{array}$ & $\begin{array}{r}14.6 \\
5.0\end{array}$ & $\begin{array}{r}22.0 \\
6.1\end{array}$ & $\begin{array}{l}5.6 \\
1.3\end{array}$ & $\begin{array}{l}8.2 \\
2.1\end{array}$ & $\begin{array}{r}14.5 \\
3.4\end{array}$ & $\begin{array}{r}9.5 \\
17.9\end{array}$ \\
\hline 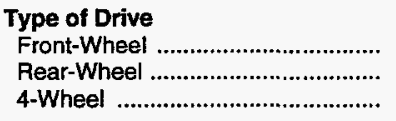 & $\begin{array}{l}38.1 \\
39.7 \\
12.7\end{array}$ & $\begin{array}{l}0.8 \\
Q \\
Q\end{array}$ & $\begin{array}{l}1.9 \\
1.8 \\
Q\end{array}$ & $\begin{array}{l}2.7 \\
3.1 \\
0.7\end{array}$ & $\begin{array}{l}6.2 \\
7.8 \\
2.0\end{array}$ & $\begin{array}{l}5.8 \\
6.9 \\
2.1\end{array}$ & $\begin{array}{l}8.2 \\
8.2 \\
3.1\end{array}$ & $\begin{array}{r}12.5 \\
11.2 \\
4.4\end{array}$ & $\begin{array}{l}3.1 \\
3.2 \\
Q\end{array}$ & $\begin{array}{l}4.3 \\
4.9 \\
1.1\end{array}$ & $\begin{array}{l}7.7 \\
8.4 \\
1.7\end{array}$ & $\begin{array}{l}10.6 \\
14.2 \\
22.6\end{array}$ \\
\hline 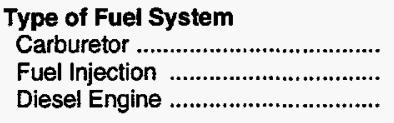 & $\begin{array}{r}43.9 \\
45.3 \\
1.4\end{array}$ & $\begin{array}{l}\mathbf{Q} \\
\mathbf{Q} \\
\mathbf{Q}\end{array}$ & $\begin{array}{l}2.7 \\
1.2 \\
Q\end{array}$ & $\begin{array}{l}4.2 \\
2.4 \\
Q\end{array}$ & $\begin{array}{l}8.9 \\
6.8 \\
Q\end{array}$ & $\begin{array}{l}7.7 \\
6.8 \\
Q\end{array}$ & $\begin{array}{r}8.5 \\
10.7 \\
Q\end{array}$ & $\begin{array}{c}10.7 \\
16.8 \\
Q\end{array}$ & $\begin{array}{l}4.7 \\
2.1 \\
Q\end{array}$ & $\begin{array}{l}6.8 \\
3.4 \\
Q\end{array}$ & $\begin{array}{l}11.2 \\
6.5 \\
Q\end{array}$ & $\begin{array}{l}12.8 \\
10.9 \\
56.9\end{array}$ \\
\hline 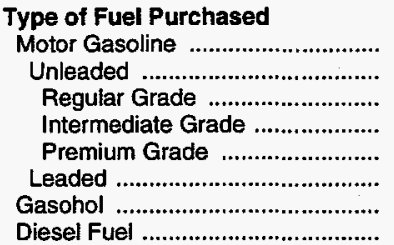 & $\begin{array}{l}88.3 \\
87.0 \\
59.9 \\
11.7 \\
15.4 \\
Q \\
0.9 \\
1.2\end{array}$ & $\begin{array}{l}1.7 \\
1.6 \\
Q \\
Q \\
Q \\
Q \\
Q \\
Q\end{array}$ & $\begin{array}{l}3.9 \\
3.8 \\
3.0 \\
Q \\
Q \\
Q \\
Q \\
Q\end{array}$ & $\begin{array}{l}6.4 \\
6.3 \\
4.6 \\
0.7 \\
1.0 \\
Q \\
Q \\
Q\end{array}$ & $\begin{array}{l}15.5 \\
15.0 \\
10.5 \\
1.7 \\
2.8 \\
Q \\
Q \\
Q\end{array}$ & $\begin{array}{l}14.3 \\
14.1 \\
9.8 \\
1.8 \\
2.6 \\
Q \\
Q \\
Q\end{array}$ & $\begin{array}{r}19.0 \\
18.9 \\
12.5 \\
3.1 \\
3.3 \\
Q \\
Q \\
Q\end{array}$ & $\begin{array}{l}27.5 \\
27.3 \\
18.4 \\
3.9 \\
4.9 \\
Q \\
Q \\
Q\end{array}$ & $\begin{array}{l}6.8 \\
6.6 \\
4.9 \\
Q \\
1.1 \\
Q \\
Q \\
Q\end{array}$ & $\begin{array}{l}9.9 \\
9.7 \\
7.0 \\
1.0 \\
1.7 \\
Q \\
Q \\
Q\end{array}$ & $\begin{array}{c}17.4 \\
17.0 \\
12.2 \\
1.8 \\
3.1 \\
Q \\
Q \\
Q\end{array}$ & $\begin{array}{r}9.2 \\
9.2 \\
10.6 \\
20.4 \\
18.3 \\
\text { NF } \\
73.9 \\
63.7\end{array}$ \\
\hline
\end{tabular}

See footnotes at end of table. 
Table 5.8. U.S. Vehicle Fuel Consumption by Family Income, 1994 (Continued) (Billion Gallons)

\begin{tabular}{|c|c|c|c|c|c|c|c|c|c|c|c|c|}
\hline \multirow{3}{*}{$\begin{array}{l}1993 \text { Household and } \\
1994 \text { Vehicle } \\
\text { Characteristics }\end{array}$} & \multirow[b]{2}{*}{ Total } & \multicolumn{7}{|c|}{1993 Family Income } & \multicolumn{2}{|c|}{$\begin{array}{l}\text { Below Poverty } \\
\text { Line }\end{array}$} & \multirow{2}{*}{$\begin{array}{l}\text { Eli- } \\
\text { gible } \\
\text { for } \\
\text { Fed- } \\
\text { eral } \\
\text { Assist- } \\
\text { ance }\end{array}$} & \multirow{3}{*}{$\begin{array}{l}\text { RSE } \\
\text { Row } \\
\text { Factor: }\end{array}$} \\
\hline & & $\begin{array}{l}\text { Less } \\
\text { than } \\
\$ 5,000\end{array}$ & $\begin{array}{l}\$ 5,000 \\
\text { to } \\
\$ 9,999\end{array}$ & $\begin{array}{l}\$ 10,000 \\
\text { to } \\
\$ 14,999\end{array}$ & $\begin{array}{c}\$ 15,000 \\
\text { to } \\
\$ 24,999\end{array}$ & $\begin{array}{c}\$ 25,000 \\
\text { to } \\
\$ 34,999\end{array}$ & $\begin{array}{c}\$ 35,000 \\
\text { to } \\
\$ 49,999\end{array}$ & $\begin{array}{l}\$ 50,000 \\
\text { or } \\
\text { More }\end{array}$ & $\begin{array}{l}100 \\
\text { Per- } \\
\text { cent }\end{array}$ & $\begin{array}{l}125 \\
\text { Per- } \\
\text { cent }\end{array}$ & & \\
\hline & 0.4 & 2.4 & 1.7 & 1.2 & 0.9 & 0.8 & 0.8 & 0.7 & 1.4 & 1.2 & 0.9 & \\
\hline 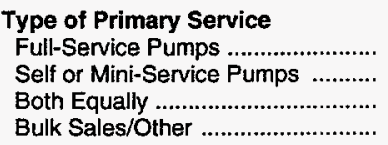 & $\begin{array}{c}6.8 \\
82.0 \\
1.6 \\
Q\end{array}$ & $\begin{array}{l}Q \\
1.5 \\
Q \\
Q\end{array}$ & $\begin{array}{l}Q \\
3.4 \\
Q \\
Q\end{array}$ & $\begin{array}{l}0.6 \\
5.9 \\
Q \\
Q\end{array}$ & $\begin{array}{c}1.0 \\
14.6 \\
Q \\
Q\end{array}$ & $\begin{array}{c}1.0 \\
13.4 \\
Q \\
Q\end{array}$ & $\begin{array}{c}1.5 \\
17.6 \\
Q \\
Q\end{array}$ & $\begin{array}{c}2.0 \\
25.6 \\
Q \\
Q\end{array}$ & $\begin{array}{l}Q \\
6.1 \\
Q \\
Q\end{array}$ & $\begin{array}{l}0.9 \\
9.2 \\
Q \\
Q\end{array}$ & $\begin{array}{c}1.7 \\
15.7 \\
Q \\
Q\end{array}$ & $\begin{array}{r}31.7 \\
9.6 \\
79.3 \\
\text { NF }\end{array}$ \\
\hline $\begin{array}{l}\text { Vehicle Used for Commuting } \\
\text { to and from Work }\end{array}$ & & & & & & & & & & & & \\
\hline Yes & $\begin{array}{l}58.2 \\
32.4\end{array}$ & $\begin{array}{l}Q \\
0.8\end{array}$ & $\begin{array}{l}2.0 \\
2.0\end{array}$ & $\begin{array}{l}3.4 \\
3.2\end{array}$ & $\begin{array}{l}9.4 \\
6.6\end{array}$ & $\begin{array}{l}9.3 \\
5.4\end{array}$ & $\begin{array}{r}12.8 \\
6.8\end{array}$ & $\begin{array}{r}20.6 \\
7.5\end{array}$ & $\begin{array}{l}3.7 \\
3.2\end{array}$ & $\begin{array}{l}5.5 \\
4.8\end{array}$ & $\begin{array}{r}10.0 \\
7.9\end{array}$ & $\begin{array}{l}10.9 \\
12.3\end{array}$ \\
\hline
\end{tabular}

1 Below 150 percent of poverty line or 60 percent of median State income.

2 Approximately 0.5 percent of the vehicle stock was owned by households that had no drivers as of fall 1993.

NF = No applicable RSE row factor.

$\mathrm{Q}=$ Data withheld either because the Relative Standard Error (RSE) was greater than 50 percent or fewer than 10 households were sampled.

Notes: "Households with Children" category includes members under age 18 years old unless the member is the householder or spouse. - To obtain the Relative Standard Error (RSE) percentage for any table cell, multiply the corresponding column and row factors. - Because of rounding, data may not sum to totals. - Data in this table are for households with vehicles for personal transportation. - See Glossary for definition of terms used in this report.

Source: Energy Information Administration, Office of Energy Markets and End Use, Form ElA-457 A of the 1993 Residential Energy Consumption Survey and Forms EIA-876 A, B, C, and D of the 1994 Residential Transportation Energy Consumption Survey. 
Table 5.9. U.S. Average Vehicle-Miles Traveled by Family Income, 1994

(Thousand Miles per Household)

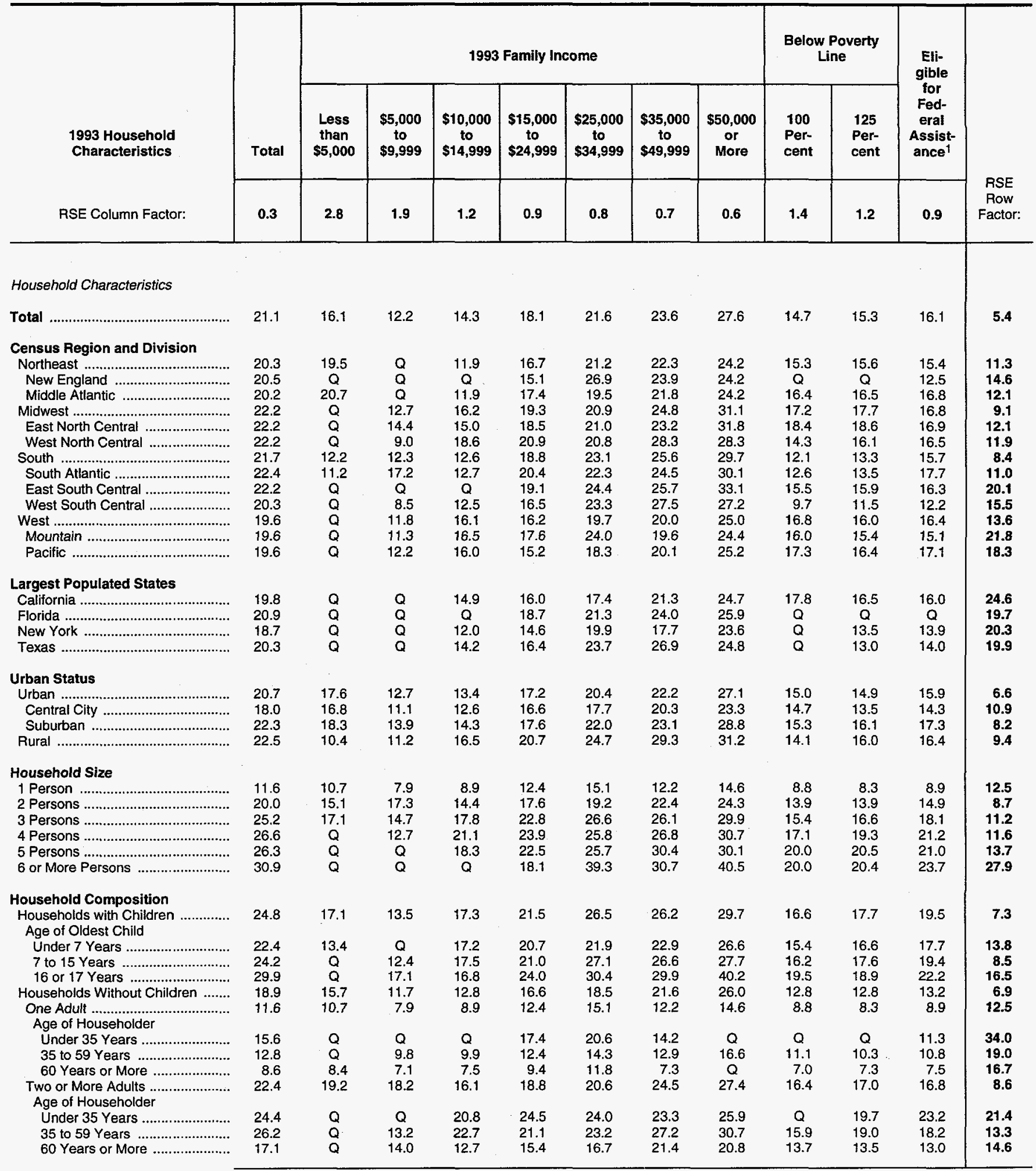

See footnotes at end of table. 
Table 5.9. U.S. Average Vehicle-Miles Traveled by Family Income, 1994 (Continued) (Thousand Miles per Household)

\begin{tabular}{|c|c|c|c|c|c|c|c|c|c|c|c|c|}
\hline \multirow[b]{2}{*}{$\begin{array}{l}1993 \text { Household } \\
\text { Characteristics }\end{array}$} & \multirow[b]{2}{*}{ Total } & \multicolumn{7}{|c|}{1993 Family Income } & \multicolumn{2}{|c|}{$\begin{array}{c}\text { Below Poverty } \\
\text { Line }\end{array}$} & \multirow{2}{*}{$\begin{array}{l}\text { Eli- } \\
\text { gible } \\
\text { for } \\
\text { Fed- } \\
\text { eral } \\
\text { Assist- } \\
\text { ance }\end{array}$} & \multirow{3}{*}{$\begin{array}{l}\text { RSE } \\
\text { Row } \\
\text { Factor: }\end{array}$} \\
\hline & & $\begin{array}{l}\text { Less } \\
\text { than } \\
\$ 5,000\end{array}$ & $\begin{array}{l}\$ 5,000 \\
\text { to } \\
\$ 9,999\end{array}$ & $\begin{array}{l}\$ 10,000 \\
\text { to } \\
\$ 14,999\end{array}$ & $\begin{array}{c}\$ 15,000 \\
\text { to } \\
\$ 24,999\end{array}$ & $\begin{array}{c}\$ 25,000 \\
\text { to } \\
\$ 34,999\end{array}$ & $\begin{array}{c}\$ 35,000 \\
\text { to } \\
\$ 49,999\end{array}$ & $\begin{array}{l}\$ 50,000 \\
\text { or } \\
\text { More }\end{array}$ & $\begin{array}{l}100 \\
\text { Per- } \\
\text { cent }\end{array}$ & $\begin{array}{l}125 \\
\text { Per- } \\
\text { cent }\end{array}$ & & \\
\hline RSE Column Factor: & 0.3 & 2.8 & 1.9 & 1.2 & 0.9 & 0.8 & 0.7 & 0.6 & 1.4 & 1.2 & 0.9 & \\
\hline \multicolumn{13}{|l|}{ Race of Householder } \\
\hline 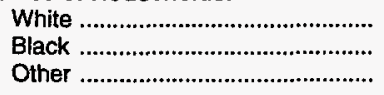 & $\begin{array}{l}21.7 \\
17.1 \\
17.8\end{array}$ & $\begin{array}{l}16.2 \\
13.8 \\
Q\end{array}$ & $\begin{array}{c}12.6 \\
10.1 \\
Q\end{array}$ & $\begin{array}{c}14.6 \\
11.5 \\
Q\end{array}$ & $\begin{array}{l}18.6 \\
14.9 \\
15.4\end{array}$ & $\begin{array}{c}21.6 \\
21.3 \\
Q\end{array}$ & $\begin{array}{l}24.0 \\
20.4 \\
18.5\end{array}$ & $\begin{array}{l}28.0 \\
25.8 \\
22.2\end{array}$ & $\begin{array}{c}15.1 \\
12.3 \\
Q\end{array}$ & $\begin{array}{l}16.2 \\
11.6 \\
14.8\end{array}$ & $\begin{array}{l}16.9 \\
12.8 \\
14.6\end{array}$ & $\begin{array}{r}5.7 \\
20.0 \\
29.9\end{array}$ \\
\hline 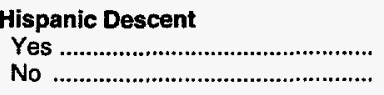 & $\begin{array}{l}19.5 \\
21.3\end{array}$ & $\stackrel{Q}{16.4}$ & $\stackrel{Q}{Q}$ & $\begin{array}{l}18.3 \\
13.8\end{array}$ & $\begin{array}{l}16.7 \\
18.2\end{array}$ & $\begin{array}{l}22.2 \\
21.5\end{array}$ & $\begin{array}{l}21.0 \\
23.8\end{array}$ & $\begin{array}{l}23.6 \\
27.9\end{array}$ & $\stackrel{Q}{14.5}$ & $\begin{array}{l}16.8 \\
15.1\end{array}$ & $\begin{array}{l}17.0 \\
16.0\end{array}$ & $\begin{array}{r}19.8 \\
5.6\end{array}$ \\
\hline 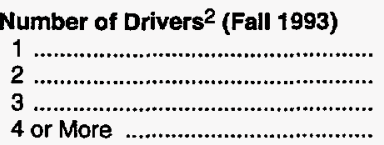 & $\begin{array}{l}12.3 \\
23.2 \\
33.1 \\
43.0\end{array}$ & $\begin{array}{l}12.6 \\
16.8 \\
Q \\
Q\end{array}$ & $\begin{array}{c}9.4 \\
20.4 \\
Q \\
Q\end{array}$ & $\begin{array}{l}10.4 \\
19.1 \\
29.0 \\
Q\end{array}$ & $\begin{array}{l}12.2 \\
21.2 \\
29.6 \\
Q\end{array}$ & $\begin{array}{l}15.6 \\
23.2 \\
29.7 \\
41.1\end{array}$ & $\begin{array}{l}12.9 \\
24.3 \\
32.5 \\
44.5\end{array}$ & $\begin{array}{l}14.9 \\
25.3 \\
37.0 \\
47.6\end{array}$ & $\begin{array}{l}11.4 \\
17.6 \\
28.2 \\
Q\end{array}$ & $\begin{array}{l}10.8 \\
18.6 \\
29.4 \\
31.0\end{array}$ & $\begin{array}{l}10.8 \\
20.0 \\
29.4 \\
35.7\end{array}$ & $\begin{array}{r}9.2 \\
6.4 \\
17.1 \\
25.1\end{array}$ \\
\hline 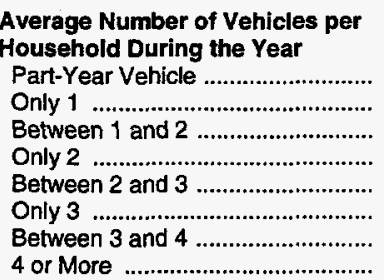 & $\begin{array}{r}5.9 \\
10.7 \\
19.5 \\
23.2 \\
29.1 \\
32.6 \\
44.3 \\
50.0\end{array}$ & $\begin{array}{c}Q \\
11.2 \\
Q \\
24.6 \\
Q \\
Q \\
Q \\
Q\end{array}$ & $\begin{array}{c}Q \\
9.1 \\
16.1 \\
19.9 \\
Q \\
Q \\
Q \\
Q\end{array}$ & $\begin{array}{c}Q \\
10.1 \\
17.6 \\
20.7 \\
Q \\
29.0 \\
Q \\
Q\end{array}$ & $\begin{array}{c}Q \\
10.1 \\
18.1 \\
21.6 \\
28.7 \\
31.4 \\
Q \\
49.2\end{array}$ & $\begin{array}{c}Q \\
12.6 \\
20.1 \\
24.1 \\
27.9 \\
33.7 \\
Q \\
53.7\end{array}$ & $\begin{array}{c}Q \\
11.2 \\
22.8 \\
23.5 \\
30.6 \\
32.7 \\
38.0 \\
45.3\end{array}$ & $\begin{array}{c}Q \\
11.9 \\
21.3 \\
24.3 \\
29.5 \\
33.1 \\
46.7 \\
50.8\end{array}$ & $\begin{array}{c}Q \\
10.7 \\
16.9 \\
22.2 \\
\mathbf{Q} \\
30.3 \\
\mathbf{Q} \\
\mathbf{Q}\end{array}$ & $\begin{array}{c}Q \\
10.3 \\
17.6 \\
22.7 \\
30.0 \\
32.6 \\
Q \\
Q\end{array}$ & $\begin{array}{c}Q \\
10.2 \\
18.5 \\
21.7 \\
29.2 \\
33.3 \\
Q \\
Q\end{array}$ & $\begin{array}{r}114.6 \\
8.3 \\
18.1 \\
6.9 \\
10.8 \\
11.7 \\
13.1 \\
16.8\end{array}$ \\
\hline
\end{tabular}

1 Below 150 percent of poverty line or 60 percent of median State income.

2 Approximately 0.5 percent of the vehicle stock was owned by households that had no drivers as of fall 1993.

$Q=$ Data witheld either because the Relative Standard Error (RSE) was greater than 50 percent or fewer than 10 households were sampled.

Notes: "Households with Children" category includes members under age 18 years old unless the member is the householder or spouse. - To obtain the Relative Standard Error (RSE) percentage for any table cell, multiply the corresponding column and row factors. - Because of rounding, data may not sum to totals. $\bullet$ Data in this table are for households with vehicles for personal transportation. - See Glossary for definition of terns used in this report.

Source: Energy Information Administration, Office of Energy Markets and End Use, Form ElA-457 A of the 1993 Residential Energy Consumption Survey and Forms

ElA-876 A, B, C, and D of the 1994 Residential Transportation Energy Consumption Survey. 
Table 5.10. U.S. Average Vehicle Fuel Consumption by Family Income, 1994 (Gallons per Household)

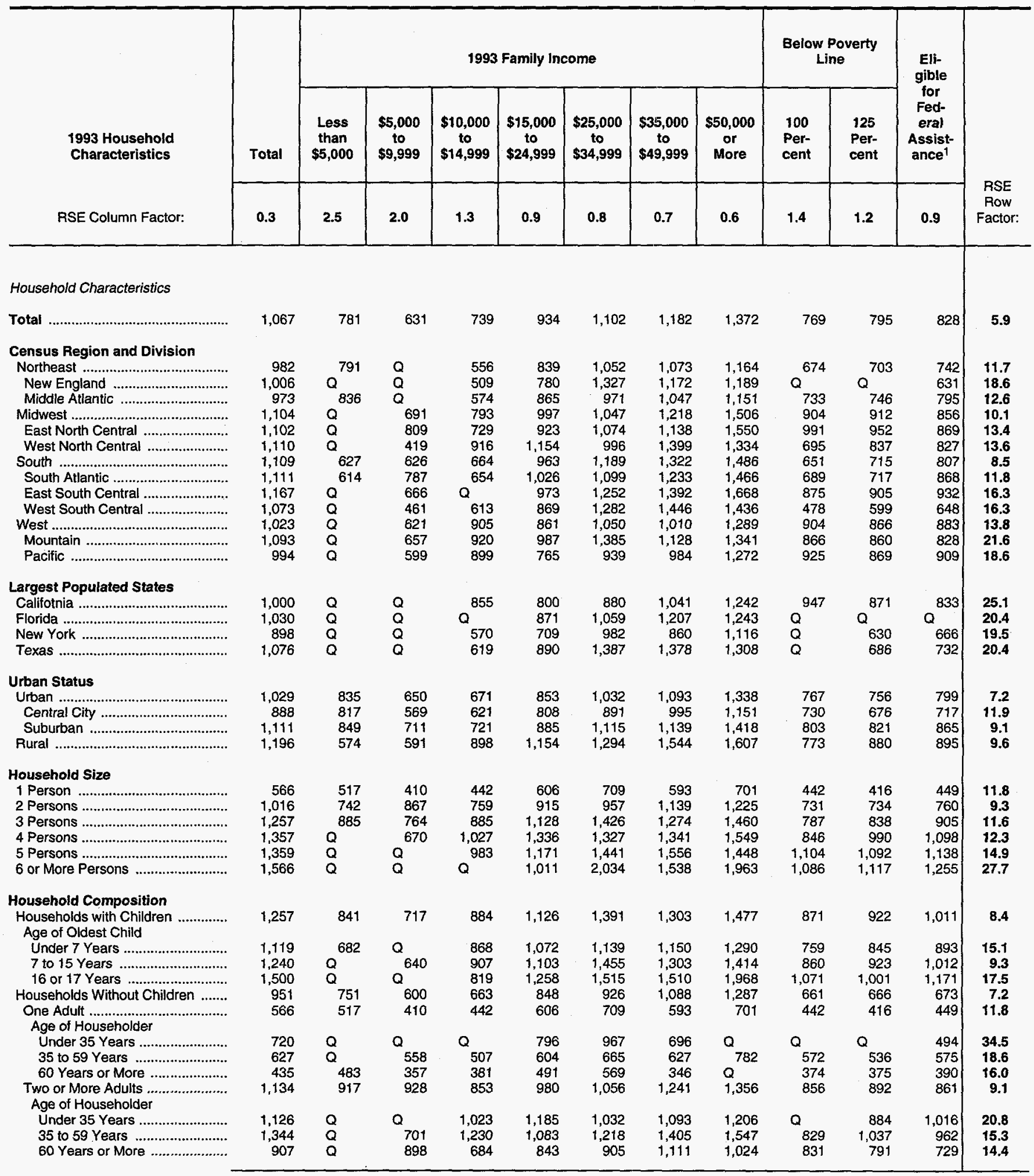

See footnotes at end of table. 
Table 5.10. U.S. Average Vehicle Fuel Consumption by Family Income, 1994 (Continued) (Gallons per Household)

\begin{tabular}{|c|c|c|c|c|c|c|c|c|c|c|c|c|}
\hline \multirow[b]{2}{*}{$\begin{array}{l}1993 \text { Household } \\
\text { Characteristics }\end{array}$} & \multirow[b]{2}{*}{ Total } & \multicolumn{7}{|c|}{1993 Family Income } & \multicolumn{2}{|c|}{$\begin{array}{c}\text { Below Poverty } \\
\text { Line }\end{array}$} & \multirow{2}{*}{$\begin{array}{c}\text { Eli- } \\
\text { gible } \\
\text { for } \\
\text { Fed- } \\
\text { eral } \\
\text { Assist- } \\
\text { ance }\end{array}$} & \multirow{3}{*}{$\begin{array}{l}\text { RSE } \\
\text { Row } \\
\text { Factor }\end{array}$} \\
\hline & & $\begin{array}{l}\text { Less } \\
\text { than } \\
\$ 5,000\end{array}$ & $\begin{array}{l}\$ 5,000 \\
\text { to } \\
\$ 9,999\end{array}$ & $\begin{array}{c}\$ 10,000 \\
\text { to } \\
\$ 14,999\end{array}$ & $\begin{array}{c}\$ 15,000 \\
\text { to } \\
\$ 24,999\end{array}$ & $\begin{array}{c}\$ 25,000 \\
\text { to } \\
\$ 34,999\end{array}$ & $\begin{array}{c}\$ 35,000 \\
\text { to } \\
\$ 49,999\end{array}$ & $\begin{array}{c}\$ 50,000 \\
\text { or } \\
\text { More }\end{array}$ & $\begin{array}{l}100 \\
\text { Per- } \\
\text { cent }\end{array}$ & $\begin{array}{l}125 \\
\text { Per- } \\
\text { cent }\end{array}$ & & \\
\hline RSE Column Factor: & 0.3 & 2.5 & 2.0 & 1.3 & 0.9 & 0.8 & 0.7 & 0.6 & 1.4 & 1.2 & 0.9 & \\
\hline \multicolumn{13}{|l|}{ Race of Househoider } \\
\hline 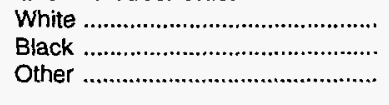 & $\begin{array}{r}1,099 \\
865 \\
867\end{array}$ & $Q^{777}$ & $\begin{array}{l}640 \\
592 \\
624\end{array}$ & $Q^{751}$ & $\begin{array}{l}963 \\
758 \\
760\end{array}$ & $Q^{1,117}$ & $\begin{array}{r}1,204 \\
1,049 \\
886\end{array}$ & $\begin{array}{l}1,395 \\
1,264 \\
1,038\end{array}$ & $Q^{778}$ & $\begin{array}{l}833 \\
655 \\
746\end{array}$ & $\begin{array}{l}862 \\
695 \\
747\end{array}$ & $\begin{array}{r}6.3 \\
20.2 \\
28.9\end{array}$ \\
\hline \multicolumn{13}{|l|}{ Hispanic Descent } \\
\hline Yes & $\begin{array}{r}990 \\
1,073\end{array}$ & $Q_{793}$ & $Q_{623}$ & $\begin{array}{l}917 \\
717\end{array}$ & $\begin{array}{l}830 \\
942\end{array}$ & $\begin{array}{l}1,212 \\
1,094\end{array}$ & $\begin{array}{l}1,073 \\
1,189\end{array}$ & $\begin{array}{l}1,157 \\
1,386\end{array}$ & $Q_{758}$ & $\begin{array}{l}853 \\
787\end{array}$ & $\begin{array}{l}860 \\
824\end{array}$ & $\begin{array}{r}21.3 \\
6.1\end{array}$ \\
\hline Number of Drivers ${ }^{2}$ (Fall 1993) & $\begin{array}{r}606 \\
1,187 \\
1,667 \\
2,071\end{array}$ & $\mathrm{Q}^{806}$ & $\begin{array}{l}492 \\
1,005 \\
\mathrm{Q} \\
\mathrm{Q}\end{array}$ & $\begin{array}{r}515 \\
995 \\
1,698 \\
Q\end{array}$ & $\begin{array}{l}606 \\
1,112 \\
1,490 \\
Q\end{array}$ & $\begin{array}{r}734 \\
1,205 \\
1,644 \\
1,999\end{array}$ & $\begin{array}{r}628 \\
1,230 \\
1,629 \\
2,146\end{array}$ & $\begin{array}{r}768 \\
1,271 \\
1,780 \\
2,282\end{array}$ & $\begin{array}{r}569 \\
955 \\
1,596 \\
Q\end{array}$ & $\begin{array}{r}534 \\
1,000 \\
1,688 \\
1,376\end{array}$ & $\begin{array}{r}543 \\
1,040 \\
1,602 \\
1,622\end{array}$ & $\begin{array}{r}9.9 \\
6.6 \\
17.6 \\
26.3\end{array}$ \\
\hline $\begin{array}{l}\text { Average Number of Vehicies per } \\
\text { Household During the Year } \\
\text { Part-Year Vehicle } \\
\text { Only } 1 \\
\text { Between } 1 \text { and } 2 \\
\text { Only } 2 \\
\text { Between } 2 \text { and } 3 \\
\text { Only } 3 \\
\text { Between } 3 \text { and } 4 \\
4 \text { or More }\end{array}$ & $\begin{array}{r}281 \\
518 \\
990 \\
1,158 \\
1,522 \\
1,726 \\
2,183 \\
2,571\end{array}$ & $\begin{array}{l}Q_{531} \\
Q^{531} \\
1,198 \\
Q \\
Q \\
Q \\
Q\end{array}$ & $\begin{array}{l}Q_{475} \\
850 \\
1,100 \\
Q \\
Q \\
Q \\
Q\end{array}$ & $\begin{array}{l}Q \\
487 \\
873 \\
1,082 \\
Q \\
1,774 \\
Q \\
Q\end{array}$ & $\begin{array}{l}Q_{492} \\
914 \\
1,119 \\
1,584 \\
1,696 \\
Q \\
2,815\end{array}$ & $\begin{array}{l}Q_{587} \\
1,022 \\
1,225 \\
1,429 \\
1,887 \\
Q \\
2,904\end{array}$ & $\begin{array}{l}Q \\
526 \\
1,131 \\
1,141 \\
1,615 \\
1,727 \\
1,936 \\
2,277\end{array}$ & $\begin{array}{l}Q_{580} \\
1,071 \\
1,182 \\
1,491 \\
1,664 \\
2,379 \\
2,556\end{array}$ & $\begin{array}{l}Q \\
531 \\
933 \\
1,157 \\
Q \\
1,782 \\
Q \\
Q\end{array}$ & $\begin{array}{l}Q \\
507 \\
936 \\
1,202 \\
1,772 \\
1,873 \\
Q \\
Q\end{array}$ & $\begin{array}{l}Q_{507} \\
501 \\
941 \\
1,151 \\
1,678 \\
1,811 \\
Q \\
Q\end{array}$ & \begin{tabular}{r|}
106.3 \\
8.1 \\
17.5 \\
7.8 \\
11.2 \\
11.3 \\
15.0 \\
15.6
\end{tabular} \\
\hline
\end{tabular}

1 Below 150 percent of poverty line or 60 percent of median State income.

2 Approximately 0.5 percent of the vehicle stock was owned by households that had no drivers as of fall 1993.

$Q=$ Data withheld either because the Relative Standard Error (RSE) was greater than 50 percent or fewer than 10 households were sampled.

Notes: "Households with Children" category includes members under age 18 years old unless the member is the householder or spouse. - To obtain the Relative Standard Error (RSE) percentage for any table cell, multiply the corresponding column and row factors. - Because of rounding, data may not sum to totals. - Data in this table are for households with vehicles for personal transportation. - See Glossary for definition of terms used in this report.

Source: Energy Information Administration, Office of Energy Markets and End Use, Form ElA-457 A of the 1993 Residential Energy Consurnption Survey and Forms EIA-876 A, B, C, and D of the 1994 Residential Transportation Energy Consumption Survey. 
Table 5.11. U.S. Vehicles by Household Composition, 1994 (Million Vehicles)

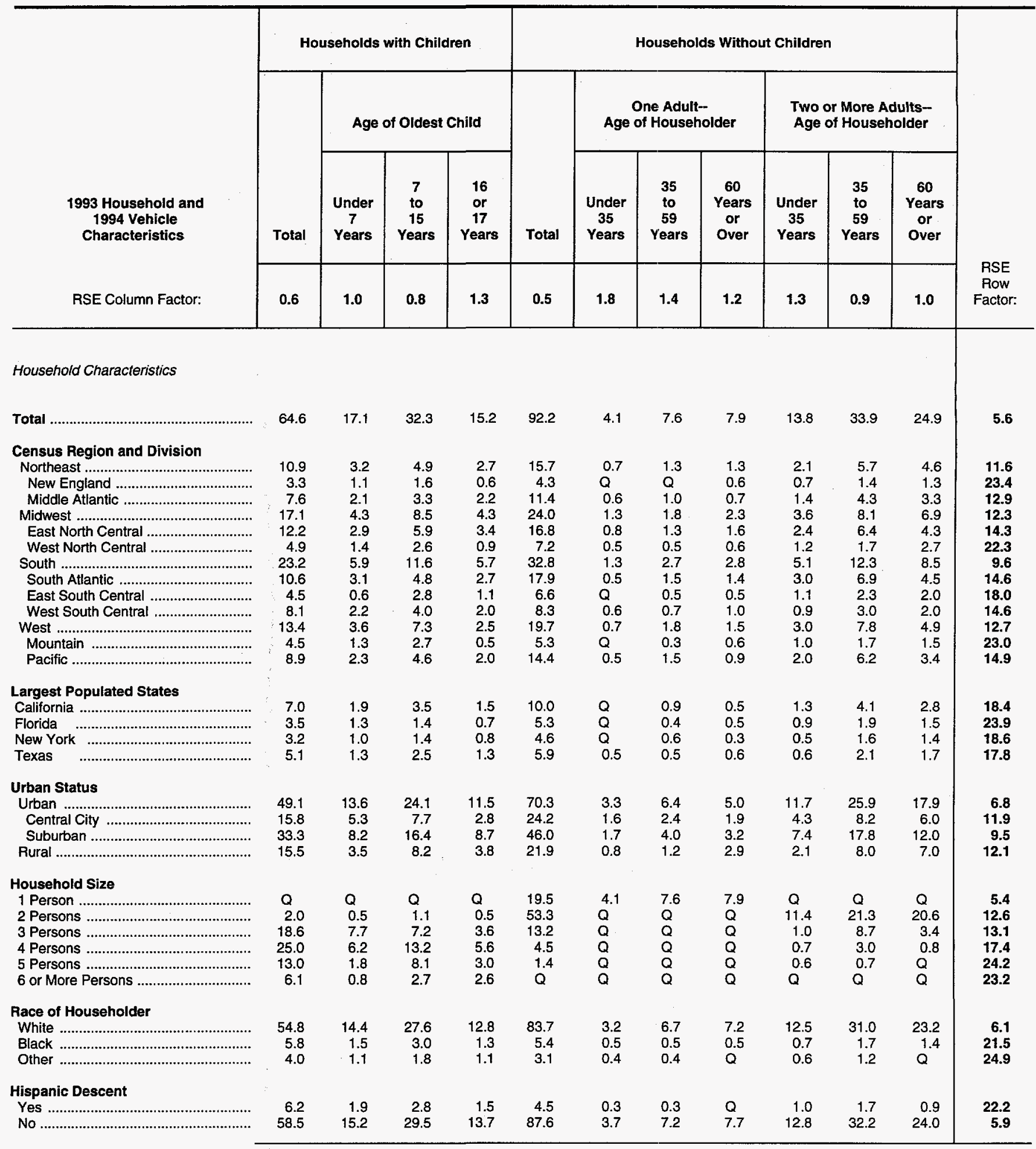

See footnotes at end of table. 
Table 5.11. U.S. Vehicles by Household Composition, 1994 (Continued) (Million Vehicles)

\begin{tabular}{|c|c|c|c|c|c|c|c|c|c|c|c|c|}
\hline \multirow[b]{3}{*}{$\begin{array}{l}1993 \text { Household and } \\
1994 \text { Vehicle } \\
\text { Characteristics }\end{array}$} & \multicolumn{4}{|c|}{ Households with Children } & \multicolumn{7}{|c|}{ Households Without Children } & \multirow{4}{*}{$\begin{array}{l}\text { RSE } \\
\text { Row } \\
\text { Factor: }\end{array}$} \\
\hline & & \multicolumn{3}{|c|}{ Age of Oldest Child } & \multirow[b]{2}{*}{ Total } & \multicolumn{3}{|c|}{$\begin{array}{l}\text { One Adult-- } \\
\text { Age of Householder }\end{array}$} & \multicolumn{3}{|c|}{$\begin{array}{l}\text { Two or More Adults- } \\
\text { Age of Householder }\end{array}$} & \\
\hline & Total & $\begin{array}{l}\text { Under } \\
7 \\
\text { Years }\end{array}$ & $\begin{array}{c}7 \\
\text { to } \\
15 \\
\text { Years }\end{array}$ & $\begin{array}{c}16 \\
\text { or } \\
17 \\
\text { Years }\end{array}$ & & $\begin{array}{l}\text { Under } \\
\mathbf{3 5} \\
\text { Years }\end{array}$ & $\begin{array}{c}35 \\
\text { to } \\
59 \\
\text { Years }\end{array}$ & $\begin{array}{l}60 \\
\text { Years } \\
\text { or } \\
\text { Over }\end{array}$ & $\begin{array}{l}\text { Under } \\
\mathbf{3 5} \\
\text { Years }\end{array}$ & $\begin{array}{c}35 \\
\text { to } \\
59 \\
\text { Years }\end{array}$ & $\begin{array}{l}60 \\
\text { Years } \\
\text { or } \\
\text { Over }\end{array}$ & \\
\hline RSE Column Factor: & 0.6 & 1.0 & 0.8 & 1.3 & 0.5 & 1.8 & 1.4 & 1.2 & 1.3 & 0.9 & 1.0 & \\
\hline 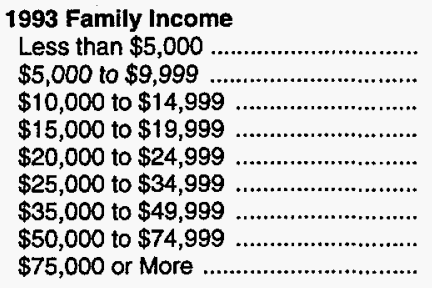 & $\begin{array}{r}1.0 \\
1.9 \\
4.4 \\
5.1 \\
4.3 \\
10.7 \\
15.6 \\
14.0 \\
7.6\end{array}$ & $\begin{array}{l}0.4 \\
0.4 \\
1.0 \\
1.5 \\
1.4 \\
2.2 \\
4.3 \\
3.7 \\
2.1\end{array}$ & $\begin{array}{l}0.3 \\
1.2 \\
2.8 \\
2.4 \\
1.9 \\
6.1 \\
7.1 \\
7.3 \\
3.3\end{array}$ & $\begin{array}{l}Q \\
0.4 \\
0.6 \\
1.1 \\
1.0 \\
2.5 \\
4.1 \\
2.9 \\
2.2\end{array}$ & $\begin{array}{r}2.0 \\
5.9 \\
8.0 \\
9.1 \\
10.1 \\
13.3 \\
18.1 \\
14.1 \\
11.5\end{array}$ & $\begin{array}{l}\mathrm{Q} \\
0.3 \\
0.4 \\
0.5 \\
0.7 \\
1.2 \\
0.6 \\
\mathrm{Q} \\
\mathrm{Q}\end{array}$ & $\begin{array}{l}Q \\
0.8 \\
0.6 \\
0.8 \\
1.4 \\
1.5 \\
1.5 \\
0.7 \\
Q\end{array}$ & $\begin{array}{l}0.3 \\
2.0 \\
1.8 \\
1.1 \\
0.7 \\
1.3 \\
0.4 \\
Q \\
Q\end{array}$ & $\begin{array}{l}1.0 \\
1.2 \\
0.9 \\
1.1 \\
1.4 \\
1.5 \\
3.1 \\
2.2 \\
1.4\end{array}$ & $\begin{array}{l}Q \\
0.6 \\
1.4 \\
2.1 \\
2.7 \\
4.1 \\
7.8 \\
8.1 \\
6.9\end{array}$ & $\begin{array}{l}Q \\
1.1 \\
3.0 \\
3.5 \\
3.1 \\
3.9 \\
4.7 \\
2.6 \\
2.8\end{array}$ & $\begin{array}{l}32.7 \\
24.2 \\
16.9 \\
18.6 \\
17.4 \\
14.8 \\
13.4 \\
15.2 \\
18.8\end{array}$ \\
\hline 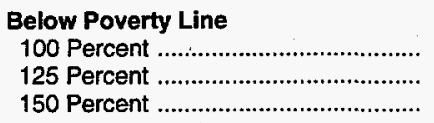 & $\begin{array}{r}6.6 \\
9.6 \\
13.7\end{array}$ & $\begin{array}{l}1.6 \\
2.1 \\
3.1\end{array}$ & $\begin{array}{l}3.6 \\
5.4 \\
7.5\end{array}$ & $\begin{array}{l}1.3 \\
2.2 \\
3.1\end{array}$ & $\begin{array}{r}5.8 \\
8.6 \\
11.9\end{array}$ & $\begin{array}{l}0.3 \\
0.3 \\
0.5\end{array}$ & $\begin{array}{l}0.7 \\
0.8 \\
1.1\end{array}$ & $\begin{array}{l}1.1 \\
1.9 \\
2.3\end{array}$ & $\begin{array}{l}1.5 \\
1.7 \\
2.7\end{array}$ & $\begin{array}{l}0.9 \\
1.7 \\
2.5\end{array}$ & $\begin{array}{l}1.4 \\
2.1 \\
2.9\end{array}$ & $\begin{array}{l}17.2 \\
15.3 \\
13.9\end{array}$ \\
\hline Eligible for Federal Assistance ${ }^{1} \ldots . .$. & 15.8 & 3.7 & 9.0 & 3.1 & 16.0 & 0.8 & 1.3 & 3.5 & 2.8 & 3.0 & 4.7 & 12.1 \\
\hline $\begin{array}{l}\text { Number of Drivers } \\
1 \\
1 \\
3\end{array}$ & $\begin{array}{r}6.6 \\
41.5 \\
11.6 \\
4.7\end{array}$ & $\begin{array}{c}1.6 \\
14.1 \\
0.9 \\
Q\end{array}$ & $\begin{array}{r}3.9 \\
24.1 \\
3.7 \\
0.6\end{array}$ & $\begin{array}{l}1.2 \\
3.4 \\
7.0 \\
3.7\end{array}$ & $\begin{array}{r}25.7 \\
50.1 \\
10.7 \\
4.9\end{array}$ & $\begin{array}{l}4.0 \\
Q \\
Q \\
Q\end{array}$ & $\begin{array}{l}7.4 \\
Q \\
Q \\
Q\end{array}$ & $\begin{array}{l}7.6 \\
Q \\
Q \\
Q\end{array}$ & $\begin{array}{r}0.7 \\
11.1 \\
0.9 \\
1.0\end{array}$ & $\begin{array}{r}2.0 \\
21.0 \\
7.8 \\
3.1\end{array}$ & $\begin{array}{r}4.1 \\
17.9 \\
2.0 \\
0.8\end{array}$ & $\begin{array}{r}10.2 \\
7.3 \\
18.7 \\
30.2\end{array}$ \\
\hline 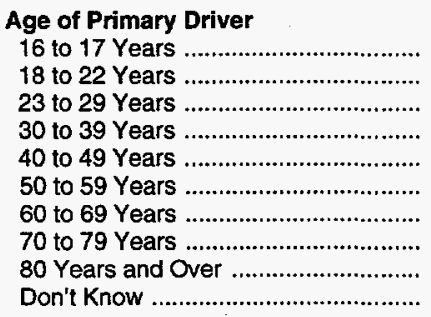 & $\begin{array}{r}1.1 \\
2.2 \\
2.8 \\
15.7 \\
13.8 \\
3.0 \\
0.7 \\
0.3 \\
Q \\
25.0\end{array}$ & $\begin{array}{l}Q \\
Q \\
1.6 \\
4.9 \\
1.6 \\
0.4 \\
Q \\
Q \\
Q \\
8.4\end{array}$ & $\begin{array}{l}0.4 \\
0.3 \\
1.1 \\
9.6 \\
7.6 \\
1.4 \\
0.4 \\
Q \\
Q \\
11.4\end{array}$ & $\begin{array}{l}0.7 \\
1.7 \\
Q \\
1.2 \\
4.7 \\
1.2 \\
Q \\
Q \\
Q \\
5.3\end{array}$ & $\begin{array}{r}\text { Q } \\
2.2 \\
5.6 \\
6.9 \\
8.4 \\
12.0 \\
9.9 \\
8.3 \\
2.5 \\
36.3\end{array}$ & $\begin{array}{l}Q \\
Q \\
1.2 \\
0.9 \\
Q \\
Q \\
Q \\
Q \\
Q \\
1.7\end{array}$ & $\begin{array}{l}Q \\
Q \\
Q \\
0.7 \\
1.8 \\
1.5 \\
Q \\
Q \\
Q \\
3.4\end{array}$ & $\begin{array}{l}Q \\
Q \\
Q \\
Q \\
Q \\
Q \\
1.6 \\
2.2 \\
0.8 \\
2.9\end{array}$ & $\begin{array}{l}\mathrm{Q} \\
0.7 \\
2.5 \\
2.7 \\
\mathrm{Q} \\
\mathrm{Q} \\
\mathrm{Q} \\
\mathrm{Q} \\
\mathrm{Q} \\
7.5\end{array}$ & $\begin{array}{l}Q \\
1.3 \\
1.4 \\
1.9 \\
5.8 \\
9.3 \\
1.2 \\
Q \\
Q \\
12.8\end{array}$ & $\begin{array}{l}Q \\
Q \\
0.4 \\
0.7 \\
0.4 \\
0.9 \\
6.9 \\
5.8 \\
1.7 \\
8.1\end{array}$ & $\begin{array}{r}29.3 \\
23.5 \\
19.1 \\
15.1 \\
14.3 \\
19.1 \\
22.0 \\
17.6 \\
20.5 \\
8.3\end{array}$ \\
\hline 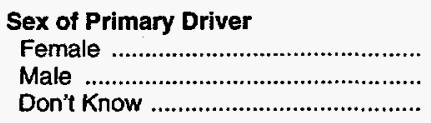 & $\begin{array}{l}18.3 \\
21.3 \\
25.0\end{array}$ & $\begin{array}{l}3.8 \\
4.9 \\
8.4\end{array}$ & $\begin{array}{r}9.8 \\
11.3 \\
11.3\end{array}$ & $\begin{array}{l}4.8 \\
5.1 \\
5.3\end{array}$ & $\begin{array}{l}25.1 \\
30.9 \\
36.1\end{array}$ & $\begin{array}{l}0.6 \\
1.7 \\
1.7\end{array}$ & $\begin{array}{l}2.0 \\
2.1 \\
3.4\end{array}$ & $\begin{array}{l}3.7 \\
1.4 \\
2.8\end{array}$ & $\begin{array}{l}2.6 \\
3.6 \\
7.5\end{array}$ & $\begin{array}{r}9.6 \\
11.6 \\
12.8\end{array}$ & $\begin{array}{r}6.5 \\
10.5 \\
8.0\end{array}$ & $\begin{array}{l}8.5 \\
9.3 \\
8.3\end{array}$ \\
\hline $\begin{array}{l}\text { Average Number of Vehicles per } \\
\text { Household During the Year } \\
\text { Part-Year Vehicle } \\
\text { Only } 1 \\
\text { Between } 1 \text { and } 2 \\
\text { Only } 2 \\
\text { Between } 2 \text { and } 3 \\
\text { Only } 3 \\
\text { Between } 3 \text { and } 4 \\
4 \text { or More }\end{array}$ & $\begin{array}{r}0.3 \\
7.4 \\
3.7 \\
25.3 \\
6.6 \\
10.4 \\
2.9 \\
7.9\end{array}$ & $\begin{array}{l}0.1 \\
2.1 \\
1.1 \\
8.9 \\
1.6 \\
1.8 \\
Q \\
1.2\end{array}$ & $\begin{array}{r}0.1 \\
3.9 \\
2.2 \\
13.3 \\
3.6 \\
4.8 \\
1.4 \\
2.9\end{array}$ & $\begin{array}{l}Q \\
1.4 \\
0.4 \\
3.1 \\
1.3 \\
3.8 \\
1.3 \\
3.8\end{array}$ & $\begin{array}{r}1.0 \\
21.2 \\
4.7 \\
31.5 \\
7.0 \\
14.8 \\
5.0 \\
6.9\end{array}$ & $\begin{array}{l}Q \\
2.5 \\
0.3 \\
0.6 \\
Q \\
Q \\
Q \\
Q\end{array}$ & $\begin{array}{l}0.3 \\
5.1 \\
0.6 \\
1.0 \\
Q \\
Q \\
Q \\
Q\end{array}$ & $\begin{array}{l}0.2 \\
5.4 \\
0.5 \\
1.2 \\
Q \\
Q \\
Q \\
Q\end{array}$ & $\begin{array}{l}0.2 \\
1.4 \\
1.0 \\
6.6 \\
1.3 \\
1.7 \\
Q \\
0.9\end{array}$ & $\begin{array}{r}0.2 \\
2.6 \\
1.3 \\
10.6 \\
3.3 \\
8.0 \\
3.4 \\
4.7\end{array}$ & $\begin{array}{r}0.1 \\
4.2 \\
1.0 \\
11.5 \\
1.9 \\
4.6 \\
0.8 \\
0.9\end{array}$ & $\begin{array}{r}33.6 \\
8.7 \\
21.2 \\
10.9 \\
18.9 \\
17.1 \\
30.8 \\
27.8\end{array}$ \\
\hline
\end{tabular}

See footnotes at end of table. 
Table 5.11. U.S. Vehicles by Household Composition, 1994 (Continued) (Million Vehicles)

\begin{tabular}{|c|c|c|c|c|c|c|c|c|c|c|c|c|}
\hline \multirow[b]{3}{*}{$\begin{array}{c}1993 \text { Household and } \\
1994 \text { Vehicle } \\
\text { Characteristics }\end{array}$} & \multicolumn{4}{|c|}{ Households with Children } & \multicolumn{7}{|c|}{ Households Without Children } & \multirow{4}{*}{$\begin{array}{l}\text { RSE } \\
\text { Row } \\
\text { Factor: }\end{array}$} \\
\hline & & \multicolumn{3}{|c|}{ Age of Oldest Child } & \multirow[b]{2}{*}{ Total } & \multicolumn{3}{|c|}{$\begin{array}{l}\text { One Adult- } \\
\text { Age of Householder }\end{array}$} & \multicolumn{3}{|c|}{$\begin{array}{l}\text { Two or More Adults-- } \\
\text { Age of Householder }\end{array}$} & \\
\hline & Total & $\begin{array}{c}\text { Under } \\
7 \\
\text { Years }\end{array}$ & $\begin{array}{c}7 \\
\text { to } \\
15 \\
\text { Years }\end{array}$ & $\begin{array}{c}16 \\
\text { or } \\
17 \\
\text { Years }\end{array}$ & & $\begin{array}{l}\text { Under } \\
35 \\
\text { Years }\end{array}$ & $\begin{array}{c}35 \\
\text { to } \\
59 \\
\text { Years }\end{array}$ & $\begin{array}{l}60 \\
\text { Years } \\
\text { or } \\
\text { Over }\end{array}$ & $\begin{array}{l}\text { Under } \\
35 \\
\text { Years }\end{array}$ & $\begin{array}{c}35 \\
\text { to } \\
59 \\
\text { Years }\end{array}$ & $\begin{array}{c}60 \\
\text { Years } \\
\text { or } \\
\text { Over }\end{array}$ & \\
\hline RSE Column Factor: & 0.6 & 1.0 & 0.8 & 1.3 & 0.5 & 1.8 & 1.4 & 1.2 & 1.3 & 0.9 & 1.0 & \\
\hline Vehicle Characteristics & & & & & & & & & & & & \\
\hline 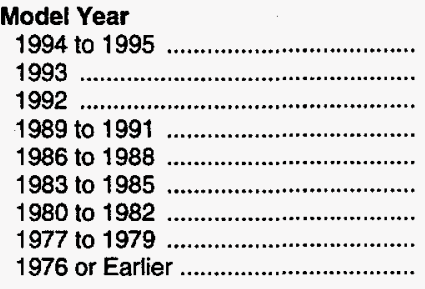 & $\begin{array}{r}3.0 \\
4.5 \\
4.8 \\
13.2 \\
14.5 \\
11.4 \\
5.0 \\
4.0 \\
4.2\end{array}$ & $\begin{array}{l}0.8 \\
1.3 \\
1.4 \\
3.6 \\
3.7 \\
2.9 \\
1.2 \\
1.0 \\
1.2\end{array}$ & $\begin{array}{l}1.5 \\
2.3 \\
2.5 \\
6.6 \\
7.0 \\
5.7 \\
2.6 \\
2.0 \\
2.1\end{array}$ & $\begin{array}{l}0.7 \\
0.9 \\
0.9 \\
3.0 \\
3.8 \\
2.8 \\
1.2 \\
0.9 \\
0.9\end{array}$ & $\begin{array}{r}4.2 \\
5.9 \\
6.5 \\
21.8 \\
21.8 \\
14.2 \\
5.9 \\
5.5 \\
6.4\end{array}$ & $\begin{array}{l}Q \\
0.4 \\
Q \\
1.0 \\
1.0 \\
0.5 \\
Q \\
Q \\
Q\end{array}$ & $\begin{array}{l}0.3 \\
0.5 \\
0.4 \\
1.9 \\
2.3 \\
1.0 \\
0.4 \\
0.4 \\
Q\end{array}$ & $\begin{array}{l}0.3 \\
0.6 \\
0.5 \\
1.8 \\
1.6 \\
1.2 \\
0.7 \\
0.6 \\
0.5\end{array}$ & $\begin{array}{l}0.9 \\
0.9 \\
0.9 \\
3.8 \\
3.1 \\
2.0 \\
0.8 \\
0.7 \\
0.8\end{array}$ & $\begin{array}{l}1.5 \\
2.2 \\
2.8 \\
7.6 \\
7.9 \\
5.5 \\
2.1 \\
1.8 \\
2.4\end{array}$ & $\begin{array}{l}0.9 \\
1.2 \\
1.8 \\
5.7 \\
5.9 \\
3.9 \\
1.7 \\
1.8 \\
2.0\end{array}$ & $\begin{array}{r}19.7 \\
15.7 \\
15.3 \\
9.7 \\
9.5 \\
11.3 \\
14.4 \\
17.6 \\
20.3\end{array}$ \\
\hline 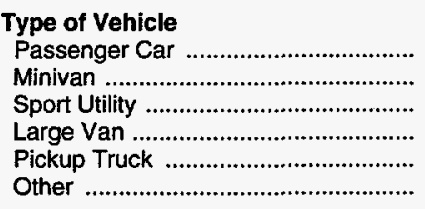 & $\begin{array}{r}39.9 \\
6.1 \\
4.8 \\
1.7 \\
12.0 \\
Q\end{array}$ & $\begin{array}{c}11.2 \\
1.3 \\
1.5 \\
Q \\
2.9 \\
Q\end{array}$ & $\begin{array}{r}18.9 \\
3.8 \\
2.5 \\
1.0 \\
6.0 \\
Q\end{array}$ & $\begin{array}{l}9.8 \\
1.1 \\
0.9 \\
0.4 \\
3.0 \\
Q\end{array}$ & $\begin{array}{r}66.6 \\
2.0 \\
4.7 \\
1.7 \\
16.8 \\
0.4\end{array}$ & $\begin{array}{l}3.0 \\
Q \\
Q \\
Q \\
0.7 \\
Q\end{array}$ & $\begin{array}{l}5.6 \\
Q \\
Q \\
Q \\
1.2 \\
Q\end{array}$ & $\begin{array}{l}7.0 \\
Q \\
Q \\
Q \\
0.8 \\
Q\end{array}$ & $\begin{array}{l}10.2 \\
Q \\
1.2 \\
Q \\
2.1 \\
Q\end{array}$ & $\begin{array}{c}22.6 \\
0.7 \\
2.2 \\
0.7 \\
7.6 \\
Q\end{array}$ & $\begin{array}{c}18.1 \\
0.8 \\
0.8 \\
0.6 \\
4.5 \\
Q\end{array}$ & $\begin{array}{r}6.0 \\
16.8 \\
15.8 \\
25.6 \\
10.5 \\
74.5\end{array}$ \\
\hline 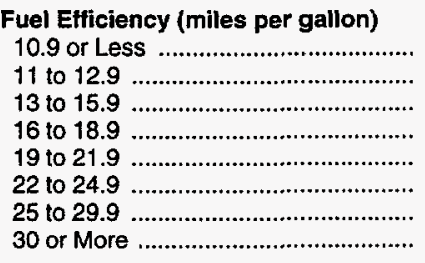 & $\begin{array}{r}2.8 \\
3.4 \\
7.7 \\
10.7 \\
14.8 \\
11.7 \\
10.6 \\
2.9\end{array}$ & $\begin{array}{l}0.8 \\
0.7 \\
2.1 \\
2.2 \\
3.7 \\
3.4 \\
3.0 \\
1.1\end{array}$ & $\begin{array}{l}1.5 \\
1.9 \\
3.8 \\
5.9 \\
7.5 \\
5.6 \\
5.0 \\
1.1\end{array}$ & $\begin{array}{l}0.5 \\
0.7 \\
1.8 \\
2.6 \\
3.6 \\
2.8 \\
2.7 \\
0.6\end{array}$ & $\begin{array}{r}4.3 \\
5.5 \\
11.1 \\
15.8 \\
20.4 \\
16.5 \\
14.3 \\
4.3\end{array}$ & $\begin{array}{l}Q \\
Q \\
0.3 \\
0.5 \\
0.6 \\
0.8 \\
1.1 \\
0.4\end{array}$ & $\begin{array}{l}Q \\
Q \\
0.9 \\
1.3 \\
1.5 \\
1.7 \\
1.5 \\
Q\end{array}$ & $\begin{array}{l}0.5 \\
0.4 \\
1.3 \\
1.4 \\
1.5 \\
1.6 \\
1.1 \\
Q\end{array}$ & $\begin{array}{l}0.3 \\
0.5 \\
0.9 \\
2.1 \\
2.8 \\
2.6 \\
3.0 \\
1.5\end{array}$ & $\begin{array}{l}1.6 \\
2.1 \\
3.9 \\
6.0 \\
7.7 \\
5.8 \\
5.2 \\
1.6\end{array}$ & $\begin{array}{l}1.4 \\
2.1 \\
3.9 \\
4.6 \\
6.3 \\
4.0 \\
2.4 \\
0.3\end{array}$ & $\begin{array}{l}20.1 \\
19.1 \\
12.5 \\
11.4 \\
10.0 \\
10.3 \\
10.2 \\
19.9\end{array}$ \\
\hline 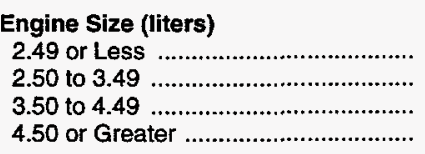 & $\begin{array}{l}24.7 \\
12.9 \\
10.1 \\
17.0\end{array}$ & $\begin{array}{l}7.5 \\
3.1 \\
2.1 \\
4.3\end{array}$ & $\begin{array}{r}11.5 \\
6.5 \\
5.5 \\
8.8\end{array}$ & $\begin{array}{l}5.7 \\
3.2 \\
2.5 \\
3.8\end{array}$ & $\begin{array}{l}35.1 \\
17.3 \\
14.0 \\
25.8\end{array}$ & $\begin{array}{l}2.2 \\
0.6 \\
0.4 \\
0.8\end{array}$ & $\begin{array}{l}3.4 \\
1.3 \\
0.9 \\
1.9\end{array}$ & $\begin{array}{l}2.8 \\
1.6 \\
1.2 \\
2.3\end{array}$ & $\begin{array}{l}7.2 \\
2.5 \\
1.7 \\
2.4\end{array}$ & $\begin{array}{r}12.5 \\
6.7 \\
5.1 \\
9.6\end{array}$ & $\begin{array}{l}6.9 \\
4.6 \\
4.7 \\
8.8\end{array}$ & $\begin{array}{r}7.6 \\
10.8 \\
11.7 \\
9.9\end{array}$ \\
\hline Number of Cylinders & $\begin{array}{r}25.8 \\
22.5 \\
15.9 \\
0.4\end{array}$ & $\begin{array}{l}7.6 \\
5.1 \\
4.2 \\
Q\end{array}$ & $\begin{array}{l}12.1 \\
12.0 \\
8.1 \\
Q\end{array}$ & $\begin{array}{l}6.1 \\
5.3 \\
3.6 \\
0\end{array}$ & $\begin{array}{r}35.8 \\
29.8 \\
25.7 \\
0.9\end{array}$ & $\begin{array}{l}2.2 \\
1.0 \\
0.8 \\
Q\end{array}$ & $\begin{array}{l}3.5 \\
2.3 \\
1.7 \\
Q\end{array}$ & $\begin{array}{l}3.1 \\
2.5 \\
2.3 \\
Q\end{array}$ & $\begin{array}{l}7.1 \\
4.1 \\
2.3 \\
Q\end{array}$ & $\begin{array}{r}12.6 \\
11.2 \\
9.8 \\
0.4\end{array}$ & $\begin{array}{l}7.2 \\
8.6 \\
8.9 \\
Q\end{array}$ & $\begin{array}{r}7.5 \\
8.3 \\
9.9 \\
44.8\end{array}$ \\
\hline 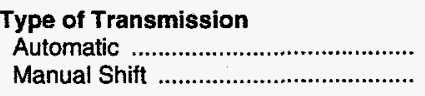 & $\begin{array}{l}47.3 \\
17.4\end{array}$ & $\begin{array}{r}12.2 \\
4.9\end{array}$ & $\begin{array}{r}23.8 \\
8.5\end{array}$ & $\begin{array}{r}11.3 \\
4.0\end{array}$ & $\begin{array}{l}70.2 \\
21.9\end{array}$ & $\begin{array}{l}2.2 \\
1.9\end{array}$ & $\begin{array}{l}5.6 \\
1.9\end{array}$ & $\begin{array}{l}7.1 \\
0.8\end{array}$ & $\begin{array}{l}8.3 \\
5.4\end{array}$ & $\begin{array}{r}25.6 \\
8.3\end{array}$ & $\begin{array}{r}21.4 \\
3.6\end{array}$ & $\begin{array}{l}6.2 \\
9.8\end{array}$ \\
\hline 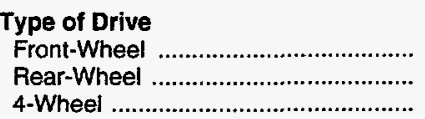 & $\begin{array}{r}31.0 \\
25.9 \\
7.7\end{array}$ & $\begin{array}{l}8.8 \\
6.0 \\
2.2\end{array}$ & $\begin{array}{r}15.1 \\
13.1 \\
4.1\end{array}$ & $\begin{array}{l}7.1 \\
6.7 \\
1.4\end{array}$ & $\begin{array}{r}43.8 \\
38.4 \\
9.9\end{array}$ & $\begin{array}{l}2.3 \\
1.1 \\
0.7\end{array}$ & $\begin{array}{l}3.8 \\
3.1 \\
0.6\end{array}$ & $\begin{array}{l}4.3 \\
3.3 \\
a\end{array}$ & $\begin{array}{l}7.1 \\
4.7 \\
2.0\end{array}$ & $\begin{array}{r}15.0 \\
14.3 \\
4.6\end{array}$ & $\begin{array}{r}11.3 \\
11.8 \\
1.8\end{array}$ & $\begin{array}{r}6.8 \\
8.1 \\
13.6\end{array}$ \\
\hline
\end{tabular}

See footnotes at end of table. 
Table 5.11. U.S. Vehicles by Household Composition, 1994 (Continued) (Million Vehicles)

\begin{tabular}{|c|c|c|c|c|c|c|c|c|c|c|c|c|}
\hline \multirow[b]{3}{*}{$\begin{array}{l}1993 \text { Household and } \\
1994 \text { Vehicle } \\
\text { Characteristics }\end{array}$} & \multicolumn{4}{|c|}{ Households with Children } & \multicolumn{7}{|c|}{ Households Without Children } & \multirow{4}{*}{$\begin{array}{l}\text { RSE } \\
\text { Row } \\
\text { Factor: }\end{array}$} \\
\hline & & \multicolumn{3}{|c|}{ Age of Oldest Child } & \multicolumn{4}{|c|}{$\begin{array}{l}\text { One Adult- } \\
\text { Age of Householder }\end{array}$} & \multicolumn{3}{|c|}{$\begin{array}{l}\text { Two or More Adults-- } \\
\text { Age of Householder }\end{array}$} & \\
\hline & Total & $\begin{array}{l}\text { Under } \\
7 \\
\text { Years }\end{array}$ & $\begin{array}{c}7 \\
\text { to } \\
15 \\
\text { Years }\end{array}$ & $\begin{array}{l}16 \\
\text { or } \\
17 \\
\text { Years }\end{array}$ & Total & $\begin{array}{l}\text { Under } \\
35 \\
\text { Years }\end{array}$ & $\begin{array}{l}35 \\
\text { to } \\
59 \\
\text { Years }\end{array}$ & $\begin{array}{l}60 \\
\text { Years } \\
\text { or } \\
\text { Over }\end{array}$ & $\begin{array}{l}\text { Under } \\
35 \\
\text { Years }\end{array}$ & $\begin{array}{c}35 \\
\text { to } \\
59 \\
\text { Years }\end{array}$ & $\begin{array}{l}60 \\
\text { Years } \\
\text { or } \\
\text { Over }\end{array}$ & \\
\hline RSE Column Factor: & 0.6 & 1.0 & 0.8 & 1.3 & 0.5 & 1.8 & 1.4 & 1.2 & 1.3 & 0.9 & 1.0 & \\
\hline \multicolumn{13}{|l|}{ Type of Fuel System } \\
\hline 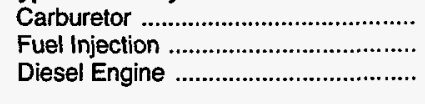 & $\begin{array}{r}32.2 \\
31.6 \\
0.9\end{array}$ & $\begin{array}{l}8.5 \\
8.4 \\
Q\end{array}$ & $\begin{array}{r}16.6 \\
15.4 \\
0.4\end{array}$ & $\begin{array}{l}7.2 \\
7.7 \\
0.3\end{array}$ & $\begin{array}{r}44.2 \\
46.7 \\
1.3\end{array}$ & $\begin{array}{l}2.0 \\
2.1 \\
Q\end{array}$ & $\begin{array}{l}3.6 \\
3.9 \\
Q\end{array}$ & $\begin{array}{l}4.1 \\
3.7 \\
Q\end{array}$ & $\begin{array}{l}6.1 \\
7.6 \\
Q\end{array}$ & $\begin{array}{r}15.6 \\
17.4 \\
0.9\end{array}$ & $\begin{array}{c}12.8 \\
11.9 \\
Q\end{array}$ & $\begin{array}{r}7.4 \\
7.0 \\
30.3\end{array}$ \\
\hline \multicolumn{13}{|l|}{ Type of Fuel Purchased } \\
\hline 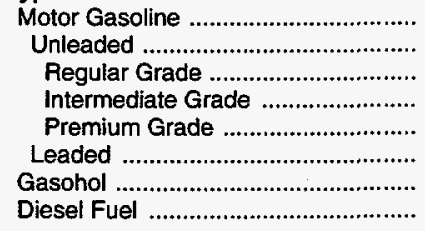 & $\begin{array}{r}63.4 \\
62.5 \\
43.1 \\
8.5 \\
10.9 \\
0.9 \\
0.5 \\
0.7\end{array}$ & $\begin{array}{l}16.8 \\
16.6 \\
11.4 \\
2.0 \\
3.2 \\
Q \\
Q \\
Q\end{array}$ & $\begin{array}{c}31.7 \\
31.1 \\
21.4 \\
4.3 \\
5.4 \\
0.5 \\
Q \\
0.3\end{array}$ & $\begin{array}{l}14.9 \\
14.8 \\
10.3 \\
2.2 \\
2.3 \\
Q \\
Q \\
Q\end{array}$ & $\begin{array}{r}90.0 \\
89.0 \\
61.1 \\
12.1 \\
15.8 \\
1.0 \\
1.0 \\
1.1\end{array}$ & $\begin{array}{l}4.0 \\
3.9 \\
2.2 \\
0.7 \\
1.1 \\
Q \\
Q \\
Q\end{array}$ & $\begin{array}{l}7.5 \\
7.4 \\
4.7 \\
1.3 \\
1.4 \\
Q \\
Q \\
Q\end{array}$ & $\begin{array}{l}7.7 \\
7.6 \\
5.3 \\
1.1 \\
1.2 \\
Q \\
Q \\
Q\end{array}$ & $\begin{array}{c}13.7 \\
13.6 \\
9.0 \\
2.1 \\
2.4 \\
Q \\
Q \\
Q\end{array}$ & $\begin{array}{c}33.1 \\
32.7 \\
22.6 \\
4.2 \\
5.9 \\
0.4 \\
Q \\
0.6\end{array}$ & $\begin{array}{c}24.1 \\
23.9 \\
17.2 \\
2.8 \\
3.9 \\
Q \\
0.6 \\
Q\end{array}$ & $\begin{array}{r}5.7 \\
5.7 \\
6.8 \\
13.3 \\
11.5 \\
40.1 \\
45.7 \\
36.4\end{array}$ \\
\hline 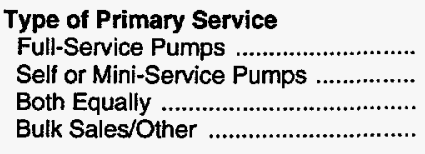 & $\begin{array}{r}4.2 \\
59.4 \\
0.9 \\
Q\end{array}$ & $\begin{array}{c}1.5 \\
15.3 \\
Q \\
Q\end{array}$ & $\begin{array}{c}1.8 \\
30.2 \\
0.3 \\
Q\end{array}$ & $\begin{array}{c}0.9 \\
13.9 \\
Q \\
Q\end{array}$ & $\begin{array}{r}9.6 \\
80.4 \\
2.0 \\
Q\end{array}$ & $\begin{array}{l}\mathrm{Q} \\
3.9 \\
\mathrm{Q} \\
\mathrm{Q}\end{array}$ & $\begin{array}{l}0.7 \\
6.7 \\
Q \\
Q\end{array}$ & $\begin{array}{l}2.6 \\
5.0 \\
0.3 \\
Q\end{array}$ & $\begin{array}{c}0.4 \\
13.2 \\
Q \\
Q\end{array}$ & $\begin{array}{c}3.0 \\
30.3 \\
0.6 \\
Q\end{array}$ & $\begin{array}{c}2.8 \\
21.4 \\
0.6 \\
Q\end{array}$ & $\begin{array}{r}21.0 \\
6.3 \\
34.7 \\
Q\end{array}$ \\
\hline \multicolumn{13}{|l|}{$\begin{array}{l}\text { Vehicle Used for Commuting } \\
\text { to and from Work }\end{array}$} \\
\hline
\end{tabular}

1 Below 150 percent of poverty line or 60 percent of median State income.

2 Approximately .5 percent of the vehicle stock was owned by households that had no drivers as of fall 1993.

NF = No applicable RSE row factor.

$Q=$ Data withheld either because the Relative Standard Error (RSE) was greater than 50 percent or fewer than 10 households were sampled.

Notes: - "Households with Children" category includesmembers under age 18 unless the member is the householder or spouse. To obtain the RSE percentage for any table cell, multiply the corresponding column and row factors. - Because of rounding, data may not sum to totals. - Data in this table are for households with vehicles for personal use. - Percentages are calculated on unrounded numbers. - See Glossary for definition of terms used in this report.

Source: Energy Information Administration, Office of Energy Markets and End Use, Form ElA-457 A of the 1993 Residential Energy Consumption Survey and Forms EIA-876 A, B, C, and D for the 1994 Residential Transportation Energy Consumption Survey. 
Table 5.12. U.S. Average Vehicle-Miles Traveled by Household Composition, 1994

(Thousand Miles per Household)

\begin{tabular}{|c|c|c|c|c|c|c|c|c|c|c|c|c|}
\hline \multirow[b]{3}{*}{$\begin{array}{l}1993 \text { Household and } \\
1994 \text { Vehicle } \\
\text { Characteristics }\end{array}$} & \multicolumn{4}{|c|}{ Households with Children } & \multicolumn{7}{|c|}{ Households without Children } & \multirow{4}{*}{$\begin{array}{l}\text { RSE } \\
\text { Row } \\
\text { Factor: }\end{array}$} \\
\hline & & \multicolumn{3}{|c|}{ Age of Oldest Child } & \multirow[b]{2}{*}{ Total } & \multicolumn{3}{|c|}{$\begin{array}{l}\text { One Adult-- } \\
\text { Age of Householder }\end{array}$} & \multicolumn{3}{|c|}{$\begin{array}{l}\text { Two or More Adults-- } \\
\text { Age of Householder }\end{array}$} & \\
\hline & Total & $\begin{array}{l}\text { Under } \\
7 \\
\text { Years }\end{array}$ & $\begin{array}{c}7 \\
\text { to } \\
15 \\
\text { Years }\end{array}$ & $\begin{array}{c}16 \\
\text { or } \\
17 \\
\text { Years }\end{array}$ & & $\begin{array}{l}\text { Under } \\
35 \\
\text { Years }\end{array}$ & $\begin{array}{c}35 \\
\text { to } \\
59 \\
\text { Years }\end{array}$ & $\begin{array}{c}60 \\
\text { Years } \\
\text { or } \\
\text { Over }\end{array}$ & $\begin{array}{l}\text { Under } \\
35 \\
\text { Years }\end{array}$ & $\begin{array}{c}35 \\
\text { to } \\
59 \\
\text { Years }\end{array}$ & $\begin{array}{c}60 \\
\text { Years } \\
\text { or } \\
\text { Over }\end{array}$ & \\
\hline RSE Column Factor: & 0.5 & 1.1 & 0.6 & 1.1 & 0.5 & 2.2 & 1.3 & 1.3 & 1.5 & 0.8 & 1.0 & \\
\hline Household Characteristics & \multicolumn{12}{|c|}{ v } \\
\hline 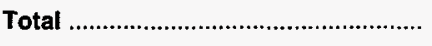 & 24.8 & 22.4 & 24.2 & 29.9 & 18.9 & 15.6 & 12.8 & 8.6 & 24,4 & 26.2 & 17.1 & 4.8 \\
\hline \multicolumn{13}{|l|}{ Census Region and Division } \\
\hline 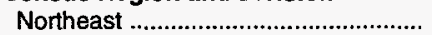 & 24.8 & 22.3 & 25.0 & 28.1 & 17.6 & $\mathrm{Q}$ & $\$ 1.0$ & 8.1 & 24.1 & 23.6 & 16.7 & 10.3 \\
\hline 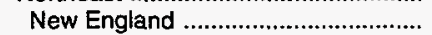 & 26.6 & 22.9 & 27.5 & $\mathrm{Q}$ & 16.9 & $\vec{Q}$ & $\mathrm{Q}$ & 8.7 & 23.7 & 25.0 & 15.5 & 20.8 \\
\hline 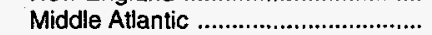 & 24.1 & 21.9 & 24.0 & 26.9 & 17.9 & $\overline{\mathbf{Q}}$ & 11.3 & 7.8 & 24.4 & 23.1 & 17.1 & 12.3 \\
\hline 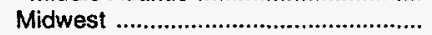 & 26.1 & 23.8 & 25.6 & 30.2 & 19.8 & 18.7 & 13.0 & 9.5 & 23.1 & 29.5 & 17.7 & 7.9 \\
\hline East North Central .................................. & 26.3 & 24.4 & 25.6 & 29.8 & 19.6 & 21.0 & 12.3 & 8.8 & 22.4 & 30.0 & 16.7 & 10.9 \\
\hline West North Central ................................ & 25.5 & 22.6 & 25.4 & 31.6 & 20.2 & 16.0 & 15.6 & 11.4 & 24.7 & 27.9 & 19.5 & 12.7 \\
\hline South ....……………………………... & 25.5 & 22.6 & 24.6 & 31.4 & 19.4 & 14.4 & 13.8 & 8.3 & 26.6 & 26.7 & 17.2 & 8.3 \\
\hline South Atlantic ...................................... & 25.1 & 23.1 & 23.8 & 30.9 & 21.0 & 15.9 & 13.5 & 9.5 & 27.5 & 28.7 & 18.0 & 11.5 \\
\hline East South Central ................................. & 28.5 & 28.0 & 26.9 & 34.4 & 18.8 & $Q$ & 14.2 & 8.0 & 28.0 & 24.3 & 16.4 & 14.2 \\
\hline West South Central ................................ & 24.6 & 20.7 & 24.5 & 30.7 & 16.8 & 12.1 & 14.1 & 6.6 & 22.7 & 24.0 & 16.5 & 17.6 \\
\hline West & 22.0 & 20.4 & 21.3 & 27.9 & 18.1 & 13.5 & 12.6 & 8.1 & 22.6 & 24.4 & 16.3 & 10.5 \\
\hline Mountain ................................................. & 22.1 & 20.1 & 22.7 & $Q$ & 17.7 & $Q$ & $Q$ & 9.7 & 20.2 & 24.3 & 15.4 & 18.0 \\
\hline 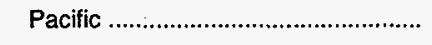 & 22.0 & 20.6 & 20.6 & 28.6 & 18.3 & 13.0 & 12.2 & 7.3 & 23.8 & 24.4 & 16.6 & 13.0 \\
\hline \multicolumn{13}{|l|}{ Largest Populated States } \\
\hline 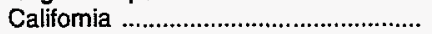 & 21.7 & 21.1 & 20.0 & 28.3 & 18.6 & $Q$ & 12.1 & 7.9 & 25.1 & 24.1 & 16.8 & 17.9 \\
\hline Florida & 25.0 & 21.3 & 25.2 & 33.2 & 18.7 & $\mathbf{Q}$ & $\mathbf{Q}$ & 9.3 & 21.5 & 25.5 & 16.2 & 23.0 \\
\hline New York & 20.7 & 18.6 & 20.5 & 24.1 & 17.5 & $Q$ & 13.5 & $\mathbf{Q}$ & 0 & 22.4 & 17.8 & 15.8 \\
\hline Texas & 24.6 & 20.4 . & 24.0 & 31.6 & 17.1 & 10.7 & $\mathbf{Q}$ & 6.6 & $Q$ & 24.4 & 18.0 & 17.7 \\
\hline \multicolumn{13}{|l|}{ Urban Status } \\
\hline 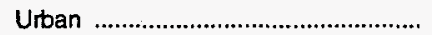 & 24.4 & 22.5 & 23.6 & 29.2 & 18.5 & 15.0 & 12.7 & 8.3 & 24.7 & 25.1 & 16.3 & 5.7 \\
\hline 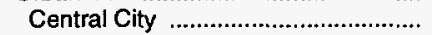 & 20.0 & 19.1 & 21.1 & 19.1 & 16.8 & 13.9 & 11.8 & 8.3 & 21.3 & 23.1 & 14.9 & 9.3 \\
\hline 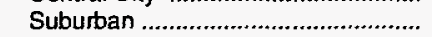 & 26.9 & 25.0 & 25.0 & 33.9 & 19.6 & 16.0 & 13.3 & 8.2 & 27.0 & 26.1 & 17.1 & 6.8 \\
\hline Rural & 26.0 & 21.7 & 25.7 & 32.0 & 20.2 & 18.7 & 13.8 & 9.2 & 22.6 & 30.1 & 19.2 & 9.0 \\
\hline \multicolumn{13}{|l|}{ Household Size } \\
\hline 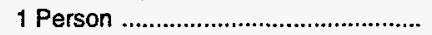 & $Q$ & $\mathbf{Q}$ & $\mathbf{Q}$ & $\mathbf{Q}$ & 11.6 & 15.6 & 12.8 & 8.6 & $Q$ & a & $\mathbf{Q}$ & 6.4 \\
\hline 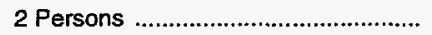 & 14.8 & 11.4 & 15.9 & 16.6 & 20.3 & $Q$ & $Q$ & $\mathbf{Q}$ & 24.0 & 23.4 & 15.6 & 9.7 \\
\hline 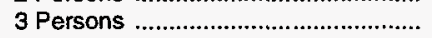 & 23.6 & 23.4 & 22.3 & 27.2 & 28.0 & $\vec{Q}$ & $\bar{Q}$ & $\bar{Q}$ & 21.6 & 30.6 & 24.7 & 11.9 \\
\hline 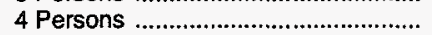 & 25.5 & 22.0 & 25.4 & 31.4 & 35.1 & $\overline{\mathrm{Q}}$ & $\bar{Q}$ & $\hat{Q}$ & $\mathrm{Q}$ & 39.0 & $\mathrm{Q}$ & 10.4 \\
\hline 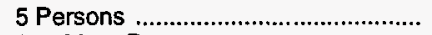 & 25.4 & 21.4 & 25.1 & 29.2 & Q & $Q$ & $\mathbf{Q}$ & $\mathbf{Q}$ & Q & $Q$ & $Q$ & 9.9 \\
\hline 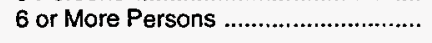 & 30.7 & $Q$ & 26.3 & 37.2 & $\vec{Q}$ & $\vec{Q}$ & $\vec{Q}$ & $\widehat{\mathbf{Q}}$ & $\vec{Q}$ & $\vec{Q}$ & $\vec{Q}$ & 20.7 \\
\hline \multicolumn{13}{|l|}{ Race of Householder } \\
\hline 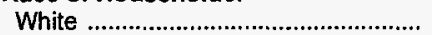 & 26.0 & 23.2 & 25.2 & 32.6 & 19.3 & 16.4 & 13.3 & 8.5 & 24.7 & 27.0 & 17.3 & 4.9 \\
\hline Black …………… & 19.1 & 18.7 & 20.5 & 16.9 & 15.3 & $Q$ & $Q$ & 8.5 & $\mathrm{Q}$ & 19.5 & 14.8 & 22.6 \\
\hline 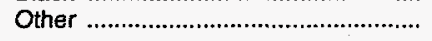 & 19.0 & 17.8 & 16.7 & 26.1 & 16.3 & $\vec{Q}$ & $\hat{Q}$ & $Q$ & 17.2 & 21.1 & $Q$ & 29.6 \\
\hline \multicolumn{13}{|l|}{ Hispanic Descent } \\
\hline 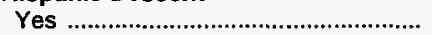 & 21.8 & 18.8 & 22.5 & 24.7 & 16.8 & 12.9 & $\mathbf{Q}$ & $\mathbf{Q}$ & 17.6 & 23.2 & 14.8 & 22.1 \\
\hline 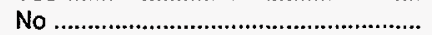 & 25.1 & 22.8 & 24.3 & 30.5 & 19.0 & 15.9 & 13.0 & 8.5 & 24.9 & 26.4 & 17.1 & 4.9 \\
\hline
\end{tabular}

See footnotes at end of table. 
Table 5.12. U.S. Average Vehicle-Miles Traveled by Household Composition, 1994 (Continued)

(Thousand Miles per Household)

\begin{tabular}{|c|c|c|c|c|c|c|c|c|c|c|c|c|}
\hline \multirow[b]{3}{*}{$\begin{array}{l}1993 \text { Household and } \\
1994 \text { Vehicle } \\
\text { Characteristics }\end{array}$} & \multicolumn{4}{|c|}{ Households with Children } & \multicolumn{7}{|c|}{ Households without Children } & \multirow{4}{*}{$\begin{array}{l}\text { RSE } \\
\text { Row } \\
\text { Factor: }\end{array}$} \\
\hline & & \multicolumn{3}{|c|}{ Age of Oldest Child } & \multicolumn{4}{|c|}{$\begin{array}{l}\text { One Adult-- } \\
\text { Age of Householder }\end{array}$} & \multicolumn{3}{|c|}{$\begin{array}{l}\text { Two or More Adults-- } \\
\text { Age of Householder }\end{array}$} & \\
\hline & Total & $\begin{array}{l}\text { Under } \\
7 \\
\text { Years }\end{array}$ & $\begin{array}{c}7 \\
\text { to } \\
15 \\
\text { Years }\end{array}$ & $\begin{array}{c}16 \\
\text { or } \\
17 \\
\text { Years }\end{array}$ & Total & $\begin{array}{l}\text { Under } \\
35 \\
\text { Years }\end{array}$ & $\begin{array}{c}35 \\
\text { to } \\
59 \\
\text { Years }\end{array}$ & $\begin{array}{l}60 \\
\text { Years } \\
\text { or } \\
\text { Over }\end{array}$ & $\begin{array}{l}\text { Under } \\
35 \\
\text { Years }\end{array}$ & $\begin{array}{c}35 \\
\text { to } \\
59 \\
\text { Years }\end{array}$ & $\begin{array}{l}60 \\
\text { Years } \\
\text { or } \\
\text { Over }\end{array}$ & \\
\hline RSE Column Factor: & 0.5 & 1.1 & 0.6 & 1.1 & 0.5 & 2.2 & 1.3 & 1.3 & 1.5 & 0.8 & 1.0 & \\
\hline 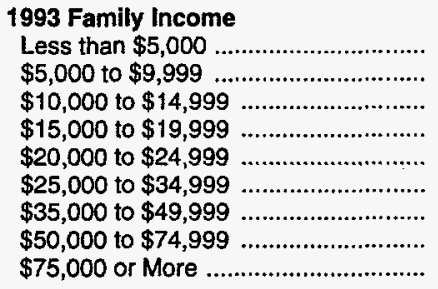 & $\begin{array}{l}17.1 \\
13.5 \\
17.3 \\
22.8 \\
20.3 \\
26.5 \\
26.2 \\
29.0 \\
30.8\end{array}$ & $\begin{array}{l}13.4 \\
Q \\
17.2 \\
21.5 \\
19.9 \\
21.9 \\
22.9 \\
27.5 \\
25.1\end{array}$ & $\begin{array}{c}Q \\
12.4 \\
17.5 \\
21.8 \\
20.0 \\
27.1 \\
26.6 \\
27.2 \\
28.9\end{array}$ & $\begin{array}{c}Q \\
17.1 \\
16.8 \\
27.6 \\
21.2 \\
30.4 \\
29.9 \\
38.4 \\
42.6\end{array}$ & $\begin{array}{l}15.7 \\
11.7 \\
12.8 \\
15.5 \\
17.6 \\
18.5 \\
21.6 \\
25.2 \\
27.1\end{array}$ & $\begin{array}{c}Q \\
Q \\
Q \\
Q \\
18.1 \\
20.6 \\
14.2 \\
Q \\
Q\end{array}$ & $\begin{array}{c}\mathrm{Q} \\
9.8 \\
9.9 \\
11.8 \\
12.7 \\
14.3 \\
12.9 \\
18.2 \\
Q\end{array}$ & $\begin{array}{r}8.4 \\
7.1 \\
7.5 \\
9.0 \\
10.0 \\
11.8 \\
7.3 \\
Q \\
Q\end{array}$ & $\begin{array}{c}Q \\
Q \\
20.8 \\
20.7 \\
28.1 \\
24.0 \\
23.3 \\
26.4 \\
25.2\end{array}$ & $\begin{array}{c}Q \\
13.2 \\
22.7 \\
18.4 \\
23.8 \\
23.2 \\
27.2 \\
29.7 \\
31.8\end{array}$ & $\begin{array}{c}Q \\
14.0 \\
12.7 \\
16.1 \\
14.6 \\
16.7 \\
21.4 \\
18.4 \\
23.3\end{array}$ & $\begin{array}{l}42.9 \\
25.2 \\
17.8 \\
18.7 \\
15.7 \\
10.6 \\
10.3 \\
10.6 \\
13.7\end{array}$ \\
\hline 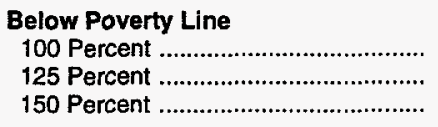 & $\begin{array}{l}16.6 \\
17.7 \\
19.0\end{array}$ & $\begin{array}{l}15.4 \\
16.6 \\
17.4\end{array}$ & $\begin{array}{l}16.2 \\
17.6 \\
18.6\end{array}$ & $\begin{array}{l}19.5 \\
18.9 \\
22.0\end{array}$ & $\begin{array}{l}12.8 \\
12.8 \\
13.6\end{array}$ & $\begin{array}{l}Q \\
Q \\
Q\end{array}$ & $\begin{array}{l}11.1 \\
10.3 \\
10.3\end{array}$ & $\begin{array}{l}7.0 \\
7.3 \\
7.4\end{array}$ & $\begin{array}{c}Q \\
19.7 \\
24.2\end{array}$ & $\begin{array}{l}15.9 \\
19.0 \\
18.2\end{array}$ & $\begin{array}{l}13.7 \\
13.5 \\
13.0\end{array}$ & $\begin{array}{l}19.2 \\
16.5 \\
13.7\end{array}$ \\
\hline Eligible for Federal Assistance ${ }^{1}$....... & 19.5 & 17.7 & 19.4 & 22.2 & 13.2 & 11.3 & 10.8 & 7.5 & 23.2 & 18.2 & 13.0 & 12.9 \\
\hline Number of Drivers ${ }^{2}$ (Fall 1993) & $\begin{array}{l}14.3 \\
24.6 \\
33.5 \\
45.2\end{array}$ & $\begin{array}{c}12.7 \\
23.9 \\
Q \\
Q\end{array}$ & $\begin{array}{c}15.1 \\
25.2 \\
34.2 \\
Q\end{array}$ & $\begin{array}{l}14.2 \\
23.1 \\
34.4 \\
45.4\end{array}$ & $\begin{array}{l}11.7 \\
22.1 \\
32.7 \\
40.8\end{array}$ & $\begin{array}{l}15.7 \\
Q \\
Q \\
Q\end{array}$ & $\begin{array}{c}13.0 \\
Q \\
Q \\
Q\end{array}$ & $\begin{array}{l}8.5 \\
Q \\
Q \\
Q\end{array}$ & $\begin{array}{c}13.9 \\
24.7 \\
26.1 \\
Q\end{array}$ & $\begin{array}{l}15.4 \\
24.2 \\
34.2 \\
43.2\end{array}$ & $\begin{array}{r}9.7 \\
18.1 \\
30.7 \\
Q\end{array}$ & $\begin{array}{r}12.0 \\
5.0 \\
13.8 \\
13.4\end{array}$ \\
\hline 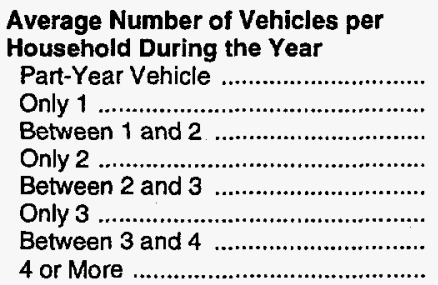 & $\begin{array}{c}Q \\
12.3 \\
20.7 \\
25.0 \\
31.2 \\
34.9 \\
45.2 \\
50.5\end{array}$ & $\begin{array}{c}Q \\
11.9 \\
18.9 \\
24.8 \\
29.2 \\
33.6 \\
Q \\
Q\end{array}$ & $\begin{array}{c}Q \\
12.3 \\
21.7 \\
24.9 \\
32.6 \\
35.3 \\
43.1 \\
45.3\end{array}$ & $\begin{array}{c}Q \\
13.0 \\
Q \\
26.1 \\
29.4 \\
35.0 \\
47.0 \\
56.1\end{array}$ & $\begin{array}{r}5.9 \\
10.2 \\
18.6 \\
21.7 \\
27.1 \\
30.9 \\
43.8 \\
49.4\end{array}$ & $\begin{array}{l}Q \\
13.0 \\
Q \\
Q \\
Q \\
Q \\
Q \\
Q\end{array}$ & $\begin{array}{c}Q \\
11.4 \\
18.1 \\
20.9 \\
Q \\
Q \\
Q \\
Q\end{array}$ & $\begin{array}{c}4.7 \\
7.2 \\
13.3 \\
15.2 \\
Q \\
Q \\
Q \\
Q\end{array}$ & $\begin{array}{c}Q \\
12.3 \\
19.0 \\
25.9 \\
29.7 \\
31.5 \\
Q \\
Q\end{array}$ & $\begin{array}{c}Q \\
12.5 \\
21.8 \\
23.9 \\
28.3 \\
32.6 \\
40.8 \\
48.4\end{array}$ & $\begin{array}{c}4.2 \\
8.7 \\
16.4 \\
17.9 \\
23.5 \\
28.2 \\
Q \\
Q\end{array}$ & $\begin{array}{r}47.6 \\
8.8 \\
16.3 \\
6.4 \\
9.5 \\
10.7 \\
14.2 \\
11.6\end{array}$ \\
\hline
\end{tabular}

1 Below 150 percent of poverty line or 60 percent of median State income.

2 Approximately .5 percent of the vehicle stock was owned by households that had no drivers as of tall 1993.

NF $=$ No applicable RSE row factor.

$Q=$ Data withheld either because the Relative Standard Error (RSE) was greater than 50 percent or fewer than 10 households were sampled.

Notes: "Households with Children" category includesmembers under age 18 unless the member is the householder or spouse.- To obtain the RSE percentage for any table cell, multiply the corresponding column and row factors. Because of rounding, data may not sum to totals. - Data in this table are for households with vehicles for personal use. - Percentages are calculated on unrounded numbers. - See Glossary for definition of terms used in this report.

Source: Energy Information Administration, Office of Energy Markets and End Use, Form ElA-457 A of the 1993 Residential Energy Consumption Survey and Forms EIA-876 A, B, C, and D for the 1994 Residential Transportation Energy Consumption Survey. 
Table 5.13. U.S. Average Vehicle-Miles Traveled by Vehicle Fuel Efficiency Category, 1994

(Thousand Miles per Vehicle)

\begin{tabular}{|c|c|c|c|c|c|c|c|c|c|c|}
\hline \multirow[b]{2}{*}{$\begin{array}{l}1993 \text { Household and } \\
1994 \text { Vehicle } \\
\text { Characteristics }\end{array}$} & \multirow[b]{2}{*}{$\begin{array}{l}\text { All Fuel- } \\
\text { Efficiency } \\
\text { Categories }\end{array}$} & \multicolumn{8}{|c|}{$\begin{array}{l}\text { Fuel Efficiency } \\
\text { (miles per gallon) }\end{array}$} & \multirow[b]{3}{*}{$\begin{array}{l}\text { RSE } \\
\text { Row } \\
\text { Factor: }\end{array}$} \\
\hline & & $\begin{array}{l}10.9 \\
\text { or } \\
\text { Less }\end{array}$ & $\begin{array}{c}11 \\
\text { to } \\
12.9\end{array}$ & $\begin{array}{c}13 \\
\text { to } \\
15.9\end{array}$ & $\begin{array}{c}16 \\
\text { to } \\
18.9\end{array}$ & $\begin{array}{l}19 \\
\text { to } \\
21.9\end{array}$ & $\begin{array}{c}22 \\
\text { to } \\
24.9\end{array}$ & $\begin{array}{l}25 \\
\text { to } \\
25.9\end{array}$ & $\begin{array}{l}30 \\
\text { or } \\
\text { More }\end{array}$ & \\
\hline RSE Column Factor: & 0.4 & 2.7 & 2.5 & 1.3 & 0.9 & 0.7 & 0.7 & 0.7 & 1.2 & \\
\hline \multicolumn{11}{|l|}{ Household Characteristics } \\
\hline Total & 11.4 & 5.6 & 8.8 & 9.7 & 10.5 & 12.0 & 12.8 & 13.0 & 14.7 & 3.9 \\
\hline \multicolumn{11}{|l|}{ Census Region and Division } \\
\hline 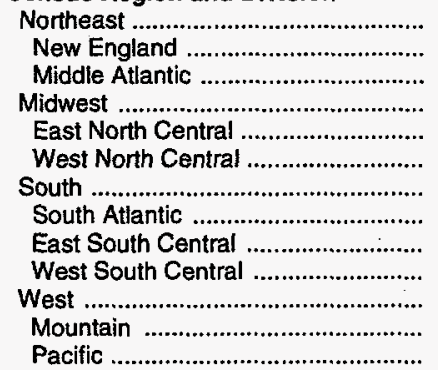 & $\begin{array}{l}11.3 \\
11.1 \\
11.3 \\
11.6 \\
11.6 \\
11.8 \\
11.7 \\
12.1 \\
10.9 \\
11.6 \\
10.9 \\
10.7 \\
10.9\end{array}$ & $\begin{array}{l}Q \\
Q \\
Q \\
6.2 \\
6.1 \\
Q \\
5.3 \\
Q \\
Q \\
4.7 \\
5.2 \\
Q \\
4.6\end{array}$ & $\begin{array}{l}8.8 \\
Q \\
Q \\
8.2 \\
7.7 \\
9.1 \\
8.7 \\
8.7 \\
Q \\
Q \\
9.4 \\
Q \\
9.5\end{array}$ & $\begin{array}{r}8.9 \\
Q \\
8.8 \\
9.3 \\
9.3 \\
9.5 \\
10.1 \\
10.1 \\
8.8 \\
10.6 \\
9.9 \\
10.7 \\
9.5\end{array}$ & $\begin{array}{r}9.7 \\
10.3 \\
9.4 \\
10.3 \\
10.5 \\
9.8 \\
11.0 \\
11.4 \\
9.8 \\
11.2 \\
10.4 \\
11.1 \\
10.1\end{array}$ & $\begin{array}{l}11.5 \\
11.8 \\
11.4 \\
12.2 \\
12.0 \\
12.7 \\
12.5 \\
12.3 \\
12.5 \\
12.8 \\
11.4 \\
10.7 \\
11.7\end{array}$ & $\begin{array}{l}12.5 \\
12.4 \\
12.5 \\
13.3 \\
13.3 \\
13.3 \\
13.1 \\
13.6 \\
11.9 \\
12.8 \\
11.8 \\
11.4 \\
12.0\end{array}$ & $\begin{array}{c}12.4 \\
Q \\
12.6 \\
13.6 \\
13.3 \\
14.4 \\
13.4 \\
13.5 \\
13.2 \\
13.5 \\
12.3 \\
13.5 \\
12.0\end{array}$ & $\begin{array}{c}14.0 \\
Q \\
Q \\
Q \\
15.0 \\
Q \\
Q \\
15.4 \\
16.0 \\
Q \\
Q \\
Q \\
Q \\
Q\end{array}$ & $\begin{array}{r}8.0 \\
17.1 \\
8.5 \\
6.6 \\
8.8 \\
10.3 \\
6.6 \\
8.7 \\
18.2 \\
11.9 \\
9.7 \\
14.4 \\
13.2\end{array}$ \\
\hline 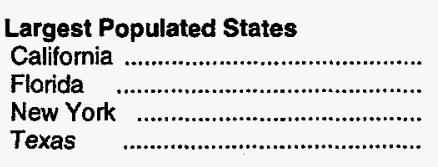 & $\begin{array}{l}11.1 \\
11.8 \\
11.4 \\
11.3\end{array}$ & $\begin{array}{l}Q \\
Q \\
Q \\
Q\end{array}$ & $\begin{array}{l}Q \\
Q \\
Q \\
Q\end{array}$ & $\begin{array}{c}9.2 \\
Q \\
Q \\
10.9\end{array}$ & $\begin{array}{r}10.2 \\
11.3 \\
9.3 \\
11.1\end{array}$ & $\begin{array}{l}12.2 \\
12.8 \\
11.4 \\
11.8\end{array}$ & $\begin{array}{c}12.3 \\
13.4 \\
13.4 \\
Q\end{array}$ & $\begin{array}{c}12.2 \\
Q \\
12.6 \\
12.8\end{array}$ & $\begin{array}{l}Q \\
Q \\
Q \\
Q\end{array}$ & $\begin{array}{r}17.9 \\
7.8 \\
13.9 \\
12.9\end{array}$ \\
\hline $\begin{array}{l}\text { Urban Status } \\
\text { Urban } \\
\text { Central City } \\
\text { Suburban } \\
\text { Rural }\end{array}$ & $\begin{array}{l}11.4 \\
10.8 \\
11.7 \\
11.6\end{array}$ & $\begin{array}{l}5.4 \\
5.6 \\
5.2 \\
6.0\end{array}$ & $\begin{array}{l}9.0 \\
8.4 \\
9.2 \\
8.6\end{array}$ & $\begin{array}{r}9.5 \\
8.9 \\
9.7 \\
10.2\end{array}$ & $\begin{array}{l}10.5 \\
10.1 \\
10.7 \\
10.5\end{array}$ & $\begin{array}{l}12.0 \\
11.4 \\
12.2 \\
12.2\end{array}$ & $\begin{array}{l}12.5 \\
11.6 \\
13.0 \\
14.0\end{array}$ & $\begin{array}{l}12.7 \\
12.3 \\
12.9 \\
14.5\end{array}$ & $\begin{array}{l}14.6 \\
13.0 \\
15.4 \\
Q\end{array}$ & $\begin{array}{l}4.8 \\
8.1 \\
5.6 \\
6.7\end{array}$ \\
\hline $\begin{array}{l}\text { Household Size } \\
1 \text { Person } \\
2 \text { Persons } \\
3 \text { Persons } \\
4 \text { Persons } \\
5 \text { Persons } \\
6 \text { or More Persons }\end{array}$ & $\begin{array}{l}10.1 \\
10.9 \\
11.9 \\
12.0 \\
12.2 \\
13.3\end{array}$ & $\begin{array}{l}5.0 \\
5.0 \\
5.2 \\
5.5 \\
Q \\
Q\end{array}$ & $\begin{array}{l}Q \\
8.6 \\
8.8 \\
9.6 \\
8.6 \\
Q\end{array}$ & $\begin{array}{r}6.9 \\
8.5 \\
11.0 \\
11.4 \\
10.5 \\
Q\end{array}$ & $\begin{array}{r}8.9 \\
9.7 \\
11.2 \\
11.4 \\
11.8 \\
Q\end{array}$ & $\begin{array}{l}10.5 \\
11.4 \\
12.1 \\
13.1 \\
12.9 \\
13.8\end{array}$ & $\begin{array}{l}11.3 \\
12.7 \\
13.2 \\
13.1 \\
13.7 \\
Q\end{array}$ & $\begin{array}{l}12.4 \\
13.0 \\
13.3 \\
12.3 \\
13.2 \\
15.3\end{array}$ & $\begin{array}{l}Q \\
15.5 \\
15.2 \\
Q \\
Q \\
Q\end{array}$ & $\begin{array}{r}12.3 \\
6.5 \\
7.9 \\
9.3 \\
11.9 \\
16.3\end{array}$ \\
\hline $\begin{array}{l}\text { Househoid Composition } \\
\text { Households with Children .................. } \\
\text { Age of Oldest Child }\end{array}$ & 12.3 & 6.4 & 9.2 & 11.3 & 11.6 & 13.2 & 13.5 & 13.3 & 14.5 & 5.7 \\
\hline 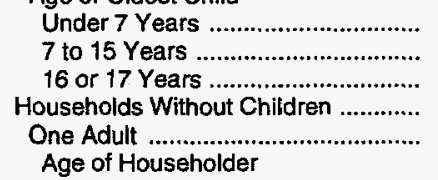 & $\begin{array}{l}12.1 \\
12.3 \\
12.5 \\
10.8 \\
10.1\end{array}$ & $\begin{array}{l}Q \\
5.7 \\
Q \\
5.0 \\
5.0\end{array}$ & $\begin{array}{l}Q \\
9.0 \\
Q \\
8.6 \\
Q\end{array}$ & $\begin{array}{r}11.4 \\
11.7 \\
10.5 \\
8.6 \\
6.9\end{array}$ & $\begin{array}{r}12.0 \\
11.7 \\
10.9 \\
9.8 \\
8.9\end{array}$ & $\begin{array}{l}12.7 \\
13.2 \\
13.7 \\
11.2 \\
10.5\end{array}$ & $\begin{array}{l}13.2 \\
13.9 \\
13.0 \\
12.4 \\
11.3\end{array}$ & $\begin{array}{l}12.4 \\
13.3 \\
14.4 \\
12.8 \\
12.4\end{array}$ & $\begin{array}{c}Q \\
14.7 \\
Q \\
14.9 \\
Q\end{array}$ & $\begin{array}{r}11.5 \\
7.9 \\
10.8 \\
5.4 \\
12.3\end{array}$ \\
\hline $\begin{array}{l}\text { Under } 35 \text { Years } \\
35 \text { to } 59 \text { Years } \\
60 \text { Years or More }\end{array}$ & $\begin{array}{r}13.0 \\
11.2 \\
7.6\end{array}$ & $\begin{array}{l}Q \\
Q \\
Q\end{array}$ & $\begin{array}{l}Q \\
Q \\
Q\end{array}$ & $\begin{array}{l}Q \\
Q \\
4.0\end{array}$ & $\begin{array}{c}Q \\
10.5 \\
7.2\end{array}$ & $\begin{array}{l}Q \\
10.7 \\
8.4\end{array}$ & $\begin{array}{c}Q \\
12.5 \\
9.7\end{array}$ & $\begin{array}{c}14.3 \\
13.3 \\
Q\end{array}$ & $\begin{array}{l}Q \\
Q \\
Q\end{array}$ & $\begin{array}{l}22.7 \\
17.7 \\
15.9\end{array}$ \\
\hline
\end{tabular}

See footnotes at end of table. 
Table 5.13. U.S. Average Vehicle-Miles Traveled by Vehicle Fuel Efficiency Category, 1994 (Continued)

(Thousand Miles per Vehicle)

\begin{tabular}{|c|c|c|c|c|c|c|c|c|c|c|}
\hline \multirow[b]{2}{*}{$\begin{array}{l}1993 \text { Household and } \\
1994 \text { Vehicle } \\
\text { Characteristics }\end{array}$} & \multirow[b]{2}{*}{$\begin{array}{l}\text { All Fuel- } \\
\text { Elficiency } \\
\text { Categories }\end{array}$} & \multicolumn{8}{|c|}{$\begin{array}{l}\text { Fuel Efficiency } \\
\text { (miles per gallon) }\end{array}$} & \multirow[b]{3}{*}{$\begin{array}{l}\text { RSE } \\
\text { Row } \\
\text { Factor: }\end{array}$} \\
\hline & & $\begin{array}{l}10.9 \\
\text { or } \\
\text { Less }\end{array}$ & $\begin{array}{c}11 \\
\text { to } \\
12.9\end{array}$ & $\begin{array}{c}13 \\
\text { to } \\
15.9\end{array}$ & $\begin{array}{c}16 \\
\text { to } \\
18.9\end{array}$ & $\begin{array}{l}19 \\
\text { to } \\
21.9\end{array}$ & $\begin{array}{c}22 \\
\text { to } \\
24.9\end{array}$ & $\begin{array}{c}25 \\
\text { to } \\
25.9\end{array}$ & $\begin{array}{l}30 \\
\text { or } \\
\text { More }\end{array}$ & \\
\hline RSE Column Factor: & 0.4 & 2.7 & 2.5 & 1.3 & 0.9 & 0.7 & 0.7 & 0.7 & 1.2 & \\
\hline \multicolumn{11}{|l|}{ Household Composition } \\
\hline $\begin{array}{l}\text { Two or More Adults .............................. } \\
\text { Age of Householder }\end{array}$ & 11.0 & 5.1 & 8.7 & 9.0 & 10.0 & 11.4 & 12.7 & 12.9 & 15.2 & 5.9 \\
\hline 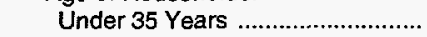 & 12.7 & 0 & $Q$ & $Q$ & 10.9 & 12.3 & 13.0 & 14.2 & 0 & 15.7 \\
\hline 35 to 59 Years ............................... & 11.6 & 6.5 & 10.4 & 9.4 & 10.9 & 11.7 & 13.2 & 13.0 & 15.3 & 7.8 \\
\hline 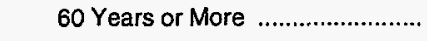 & 9.3 & 3.1 & 7.0 & 7.9 & 8.3 & 10.5 & 11.8 & 11.0 & $Q$ & 9.8 \\
\hline \multicolumn{11}{|l|}{ Race of Householder } \\
\hline White ............................ & 11.5 & 5.6 & 8.8 & 9.7 & 10.5 & 12.1 & 12.9 & 13.1 & 14.9 & 4.1 \\
\hline Black ……........................... & 11.2 & $Q$ & $\mathbf{Q}$ & $Q$ & 10.2 & 11.6 & 12.1 & 13.6 & $Q$ & 15.2 \\
\hline Other & 10.9 & $\mathbf{Q}$ & $Q$ & $Q$ & $\mathrm{Q}$ & $Q$ & $Q$ & 11.1 & $\bar{Q}$ & 16.9 \\
\hline \multicolumn{11}{|l|}{ Hispanic Descent } \\
\hline Yes ............................ & 11.4 & $\mathrm{Q}$ & $Q$ & $Q$ & 10.3 & 11.9 & 12.0 & $\mathrm{Q}$ & $\mathbf{Q}$ & 18.4 \\
\hline No & 11.4 & 5.5 & 8.9 & 9.6 & 10.5 & 12.0 & 12.9 & 13.0 & 14.8 & 4.0 \\
\hline \multicolumn{11}{|l|}{1993 family Income } \\
\hline Less than $\$ 5,000$ & 11.5 & 0 & $\mathrm{Q}$ & $Q$ & a & $Q$ & $Q$ & $Q$ & $Q$ & 22.4 \\
\hline$\$ 5,000$ to $\$ 9,999$ & 9.9 & $Q$ & $Q$ & 7.7 & 9.3 & $\mathrm{Q}$ & $\mathrm{Q}$ & $\mathrm{Q}$ & $Q$ & 22.4 \\
\hline$\$ 10,000$ to $\$ 14,999$. & 10.2 & 5.3 & $Q$ & 7.4 & 9.3 & 11.5 & 11.3 & 13.2 & $Q$ & 13.9 \\
\hline$\$ 15,000$ to $\$ 19,999$ & 10.7 & $\mathbf{Q}$ & $Q$ & 9.0 & 9.5 & 11.4 & 12.0 & 12.2 & $\widehat{Q}$ & 14.4 \\
\hline$\$ 20,000$ to $\$ 24,999$ & 11.0 & $Q$ & 8.2 & 8.7 & 11.0 & 10.9 & 11.9 & 13.5 & $Q$ & 14.7 \\
\hline$\$ 25,000$ to $\$ 34,999 \ldots \ldots \ldots \ldots \ldots \ldots \ldots \ldots$ & 11.9 & 4.7 & 10.8 & 10.6 & 10.8 & 12.6 & 12.5 & 14.2 & $\bar{Q}$ & 8.8 \\
\hline$\$ 35,000$ to $\$ 49,999$ & 11.6 & 4.5 & 9.4 & 10.7 & 10.4 & 11.8 & 13.1 & 12.8 & 14.9 & 7.6 \\
\hline$\$ 50,000$ to $\$ 74,999$ & 11.8 & $\mathrm{Q}$ & 6.4 & 9.3 & 11.2 & 12.7 & 13.6 & 13.2 & 14.1 & 8.4 \\
\hline$\$ 75,000$ or More & 12.3 & $\mathrm{O}$ & $Q$ & 11.4 & 10.8 & 12.8 & 13.9 & 12.7 & $\mathrm{Q}$ & 10.7 \\
\hline \multicolumn{11}{|l|}{ Below Poverty Line } \\
\hline 100 Percent .................. & 10.7 & $\mathrm{Q}$ & $\mathbf{Q}$ & 8.8 & 10.2 & 10.9 & 12.0 & $Q$ & $Q$ & 18.6 \\
\hline 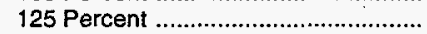 & 10.9 & 0 & 8.3 & 9.2 & 10.3 & 11.3 & 12.2 & 12.6 & $\vec{Q}$ & 12.3 \\
\hline 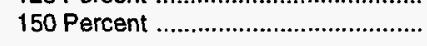 & 11.1 & 7.1 & 8.6 & 9.4 & 10.3 & 11.6 & 12.3 & 12.9 & 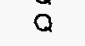 & 11.0 \\
\hline Eligible for Federal Assistance ${ }^{1}$....... & 10.9 & 6.6 & 8.4 & 8.9 & 10.2 & 11.4 & 12.1 & 12.8 & 15.5 & 10.4 \\
\hline \multicolumn{11}{|l|}{ Number of Drivers ${ }^{2}$ (Fall 1993) } \\
\hline 1 & 10.3 & 5.2 & 8.1 & 7.2 & 8.9 & 11.2 & 11.4 & 13.0 & Q & 9.3 \\
\hline 2 & 11.6 & 5.4 & 8.9 & 10.2 & 10.8 & 12.2 & 13.2 & 12.9 & 15.1 & 5.0 \\
\hline 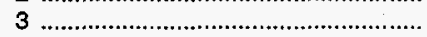 & 12.0 & $Q$ & 10.7 & 11.1 & 10.9 & 12.3 & 13.1 & 12.8 & 15.2 & 10.0 \\
\hline 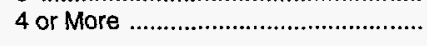 & 12.8 & $Q$ & $Q$ & $Q$ & 12.4 & 12.4 & 14.1 & 14.6 & $Q$ & 15.8 \\
\hline \multicolumn{11}{|l|}{ Age of Primary Driver } \\
\hline 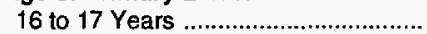 & 9.6 & 0 & $Q$ & $Q$ & $Q$ & $Q$ & $Q$ & $Q$ & $Q$ & 56.2 \\
\hline 18 to 22 Years ...................................... & 12.9 & $Q$ & $Q$ & $Q$ & $\bar{Q}$ & $\bar{Q}$ & 12.1 & 14.7 & $\mathbf{Q}$ & 16.6 \\
\hline 23 to 29 Years ..................................... & 13.3 & 0 & $Q$ & $\bar{Q}$ & 8.4 & 14.5 & 13.6 & 16.7 & $\vec{Q}$ & 12.5 \\
\hline 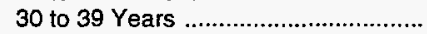 & 12.2 & 6.1 & 8.6 & 11.7 & 11.2 & 12.6 & 13.9 & 13.2 & 16.1 & 8.1 \\
\hline 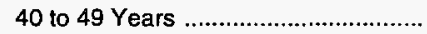 & 12.4 & 3.8 & 9.6 & 10.8 & 11.5 & 13.6 & 13.8 & 13.4 & $Q$ & 7.6 \\
\hline 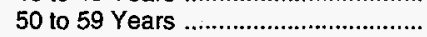 & 11.6 & 6.1 & 10.4 & 8.6 & 9.8 & 12.0 & 15.3 & 13.8 & $Q$ & 11.0 \\
\hline 60 to 69 Years & 9.3 & 3.7 & 4.5 & 10.0 & 8.4 & 10.7 & 11.5 & 10.7 & $\bar{Q}$ & 12.4 \\
\hline 70 to 79 Years & 7.9 & 2.7 & $Q$ & 4.9 & 7.4 & 9.8 & 9.1 & 11.8 & $Q$ & 13.4 \\
\hline 80 Years and Over & 6.1 & $Q$ & $\mathbf{Q}$ & 2.9 & $\mathrm{Q}$ & $Q$ & $\mathrm{Q}$ & $Q$ & $\mathrm{Q}$ & 26.7 \\
\hline 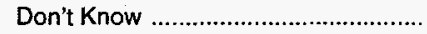 & 11.5 & $\mathrm{Q}$ & 9.0 & 10.6 & 11.3 & 11.7 & 12.1 & 12.3 & $\mathbf{Q}$ & 10.5 \\
\hline
\end{tabular}

See footnotes at end of table. 
Table 5.13. U.S. Average Vehicle-Miles Traveled by Vehicle Fuel Efficiency Category, 1994 (Continued)

(Thousand Miles per Vehicle)

\begin{tabular}{|c|c|c|c|c|c|c|c|c|c|c|}
\hline \multirow[b]{2}{*}{$\begin{array}{l}1993 \text { Household and } \\
1994 \text { Vehicle } \\
\text { Characteristics }\end{array}$} & \multirow[b]{2}{*}{$\begin{array}{l}\text { All Fuel- } \\
\text { Efficiency } \\
\text { Categories }\end{array}$} & \multicolumn{8}{|c|}{$\begin{array}{l}\text { Fuel Efficiency } \\
\text { (miles per gallon) }\end{array}$} & \multirow[b]{3}{*}{$\begin{array}{l}\text { RSE } \\
\text { Row } \\
\text { Factor: }\end{array}$} \\
\hline & & $\begin{array}{l}10.9 \\
\text { or } \\
\text { Less }\end{array}$ & $\begin{array}{c}11 \\
\text { to } \\
12.9\end{array}$ & $\begin{array}{c}13 \\
\text { to } \\
15.9\end{array}$ & $\begin{array}{c}16 \\
\text { to } \\
18.9\end{array}$ & $\begin{array}{c}19 \\
10 \\
21.9\end{array}$ & $\begin{array}{c}22 \\
10 \\
24.9\end{array}$ & $\begin{array}{c}25 \\
\text { to } \\
25.9\end{array}$ & $\begin{array}{l}30 \\
\text { or } \\
\text { More }\end{array}$ & \\
\hline RSE Column Factor: & 0.4 & 2.7 & 2.5 & 1.3 & 0.9 & 0.7 & 0.7 & 0.7 & 1.2 & \\
\hline \multicolumn{11}{|l|}{ Sex of Primary Driver } \\
\hline 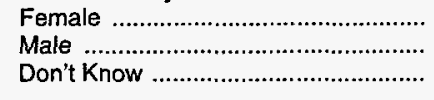 & $\begin{array}{l}11.7 \\
11.1 \\
11.5\end{array}$ & $\begin{array}{l}Q \\
4.7 \\
Q\end{array}$ & $\begin{array}{l}6.2 \\
9.4 \\
9.0\end{array}$ & $\begin{array}{r}8.0 \\
9.7 \\
10.6\end{array}$ & $\begin{array}{r}9.6 \\
10.2 \\
11.4\end{array}$ & $\begin{array}{l}11.5 \\
13.0 \\
11.7\end{array}$ & $\begin{array}{l}13.2 \\
13.4 \\
12.2\end{array}$ & $\begin{array}{l}13.7 \\
13.4 \\
12.3\end{array}$ & $\begin{array}{c}16.3 \\
15.6 \\
Q\end{array}$ & $\begin{array}{r}6.3 \\
5.4 \\
10.4\end{array}$ \\
\hline $\begin{array}{l}\text { Average Number of Vehicles per } \\
\text { Household During the Year } \\
\text { Part-Year Vehicle } \\
\text { Only } 1 \\
\text { Between } 1 \text { and } 2 \\
\text { Only } 2 \\
\text { Between } 2 \text { and } 3 \\
\text { Only } 3 \\
\text { Between } 3 \text { and } 4 \\
4 \text { or More }\end{array}$ & $\begin{array}{c}Q \\
10.7 \\
12.6 \\
11.6 \\
12.0 \\
10.9 \\
12.7 \\
11.4\end{array}$ & $\begin{array}{l}\mathrm{Q} \\
\mathrm{Q} \\
\mathrm{Q} \\
6.0 \\
\mathrm{Q} \\
5.2 \\
\mathrm{Q} \\
\mathrm{Q}\end{array}$ & $\begin{array}{l}\mathrm{Q} \\
\mathrm{Q} \\
\mathrm{Q} \\
8.9 \\
8.2 \\
9.4 \\
\mathrm{Q} \\
\mathrm{Q}\end{array}$ & $\begin{array}{c}Q \\
6.9 \\
11.9 \\
10.2 \\
10.3 \\
9.8 \\
Q \\
10.0\end{array}$ & $\begin{array}{r}\mathrm{Q} \\
9.5 \\
11.0 \\
10.6 \\
11.7 \\
9.4 \\
14.2 \\
10.3\end{array}$ & $\begin{array}{c}Q \\
11.4 \\
13.7 \\
11.9 \\
12.4 \\
12.1 \\
12.1 \\
12.4\end{array}$ & $\begin{array}{c}Q \\
11.8 \\
14.1 \\
12.9 \\
13.8 \\
12.9 \\
13.5 \\
12.7\end{array}$ & $\begin{array}{c}Q \\
12.7 \\
13.3 \\
12.7 \\
14.8 \\
12.5 \\
Q \\
14.6\end{array}$ & $\begin{array}{c}Q \\
Q \\
Q \\
14.6 \\
Q \\
Q \\
Q \\
Q\end{array}$ & $\begin{array}{r}100.0 \\
9.9 \\
14.8 \\
6.5 \\
10.2 \\
9.0 \\
18.1 \\
12.0\end{array}$ \\
\hline \multicolumn{11}{|l|}{ Vehicle Characteristics } \\
\hline 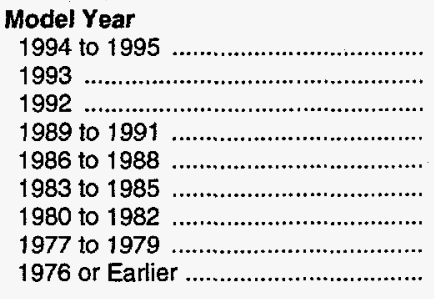 & $\begin{array}{r}14.3 \\
13.7 \\
13.1 \\
12.4 \\
11.3 \\
10.6 \\
9.8 \\
9.3 \\
8.2\end{array}$ & $\begin{array}{l}\mathrm{Q} \\
\mathrm{Q} \\
\mathrm{Q} \\
\mathrm{Q} \\
\mathrm{Q} \\
\mathrm{Q} \\
\mathrm{Q} \\
4.2 \\
6.5\end{array}$ & $\begin{array}{c}Q \\
Q \\
Q \\
11.6 \\
5.4 \\
5.9 \\
Q \\
9.8 \\
10.5\end{array}$ & $\begin{array}{r}12.0 \\
11.4 \\
Q \\
10.6 \\
9.4 \\
8.7 \\
8.4 \\
10.7 \\
8.2\end{array}$ & $\begin{array}{r}13.3 \\
12.5 \\
12.3 \\
11.0 \\
9.0 \\
9.8 \\
10.1 \\
Q \\
Q\end{array}$ & $\begin{array}{c}14.2 \\
13.8 \\
12.8 \\
12.2 \\
11.6 \\
11.6 \\
10.0 \\
Q \\
Q\end{array}$ & $\begin{array}{c}15.8 \\
14.5 \\
14.5 \\
13.3 \\
12.0 \\
10.5 \\
Q \\
Q \\
Q\end{array}$ & $\begin{array}{c}15.1 \\
14.7 \\
13.7 \\
13.3 \\
12.4 \\
12.5 \\
Q \\
Q \\
Q\end{array}$ & $\begin{array}{c}Q \\
Q \\
Q \\
Q \\
15.1 \\
Q \\
Q \\
Q \\
Q\end{array}$ & $\begin{array}{r}12.1 \\
12.1 \\
10.2 \\
7.3 \\
8.7 \\
10.4 \\
17.6 \\
10.7 \\
12.2\end{array}$ \\
\hline 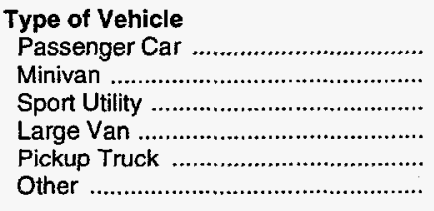 & $\begin{array}{l}11.3 \\
13.4 \\
12.7 \\
11.7 \\
11.1 \\
Q\end{array}$ & $\begin{array}{l}4.9 \\
Q \\
4.1 \\
Q \\
5.6\end{array}$ & $\begin{array}{l}7.4 \\
Q \\
Q \\
Q \\
9.8 \\
Q\end{array}$ & $\begin{array}{l}7.2 \\
Q \\
12.5 \\
12.5 \\
11.1 \\
Q\end{array}$ & $\begin{array}{c}8.6 \\
11.2 \\
13.5 \\
Q \\
12.5 \\
Q\end{array}$ & $\begin{array}{l}11.2 \\
15.2 \\
15.8 \\
Q \\
12.1 \\
Q\end{array}$ & $\begin{array}{c}12.7 \\
Q \\
Q \\
Q \\
13.1 \\
Q\end{array}$ & $\begin{array}{c}12.9 \\
Q \\
Q \\
Q \\
15.0 \\
Q\end{array}$ & $\begin{array}{l}14.8 \\
Q \\
Q \\
Q \\
Q \\
Q\end{array}$ & $\begin{array}{r}5.3 \\
7.1 \\
9.9 \\
11.7 \\
8.1 \\
100.0\end{array}$ \\
\hline 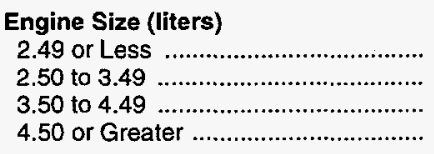 & $\begin{array}{l}11.7 \\
12.1 \\
11.7 \\
10.5\end{array}$ & $\begin{array}{l}\mathrm{Q} \\
\mathrm{Q} \\
\mathrm{Q} \\
5.8\end{array}$ & $\begin{array}{l}Q \\
Q \\
4.9 \\
9.6\end{array}$ & $\begin{array}{r}2.8 \\
5.1 \\
8.1 \\
11.1\end{array}$ & $\begin{array}{r}6.2 \\
9.7 \\
11.4 \\
11.3\end{array}$ & $\begin{array}{r}9.3 \\
12.3 \\
12.9 \\
14.3\end{array}$ & $\begin{array}{c}11.3 \\
14.5 \\
17.0 \\
Q\end{array}$ & $\begin{array}{c}12.9 \\
Q \\
Q \\
Q\end{array}$ & $\begin{array}{l}14.7 \\
Q \\
Q \\
Q\end{array}$ & $\begin{array}{l}7.2 \\
7.4 \\
9.4 \\
5.5\end{array}$ \\
\hline Number of Cylinders & $\begin{array}{l}11.6 \\
12.1 \\
10.5 \\
10.6\end{array}$ & $\begin{array}{l}Q \\
3.6 \\
5.9 \\
Q\end{array}$ & $\begin{array}{l}Q \\
6.5 \\
9.4 \\
Q\end{array}$ & $\begin{array}{c}3.3 \\
7.7 \\
11.2 \\
Q\end{array}$ & $\begin{array}{c}6.8 \\
11.2 \\
10.8 \\
Q\end{array}$ & $\begin{array}{r}9.2 \\
12.7 \\
14.1 \\
Q\end{array}$ & $\begin{array}{c}11.3 \\
15.5 \\
Q \\
Q\end{array}$ & $\begin{array}{c}13.0 \\
Q \\
Q \\
Q\end{array}$ & $\begin{array}{l}14.8 \\
Q \\
Q \\
Q\end{array}$ & $\begin{array}{r}6.7 \\
6.9 \\
5.6 \\
51.8\end{array}$ \\
\hline
\end{tabular}

See footnotes at end of table. 
Table 5.13. U.S. Average Vehicle-Miles Traveled by Vehicle Fuel Efficiency Category, 1994 (Continued)

(Thousand Miles per Vehicle)

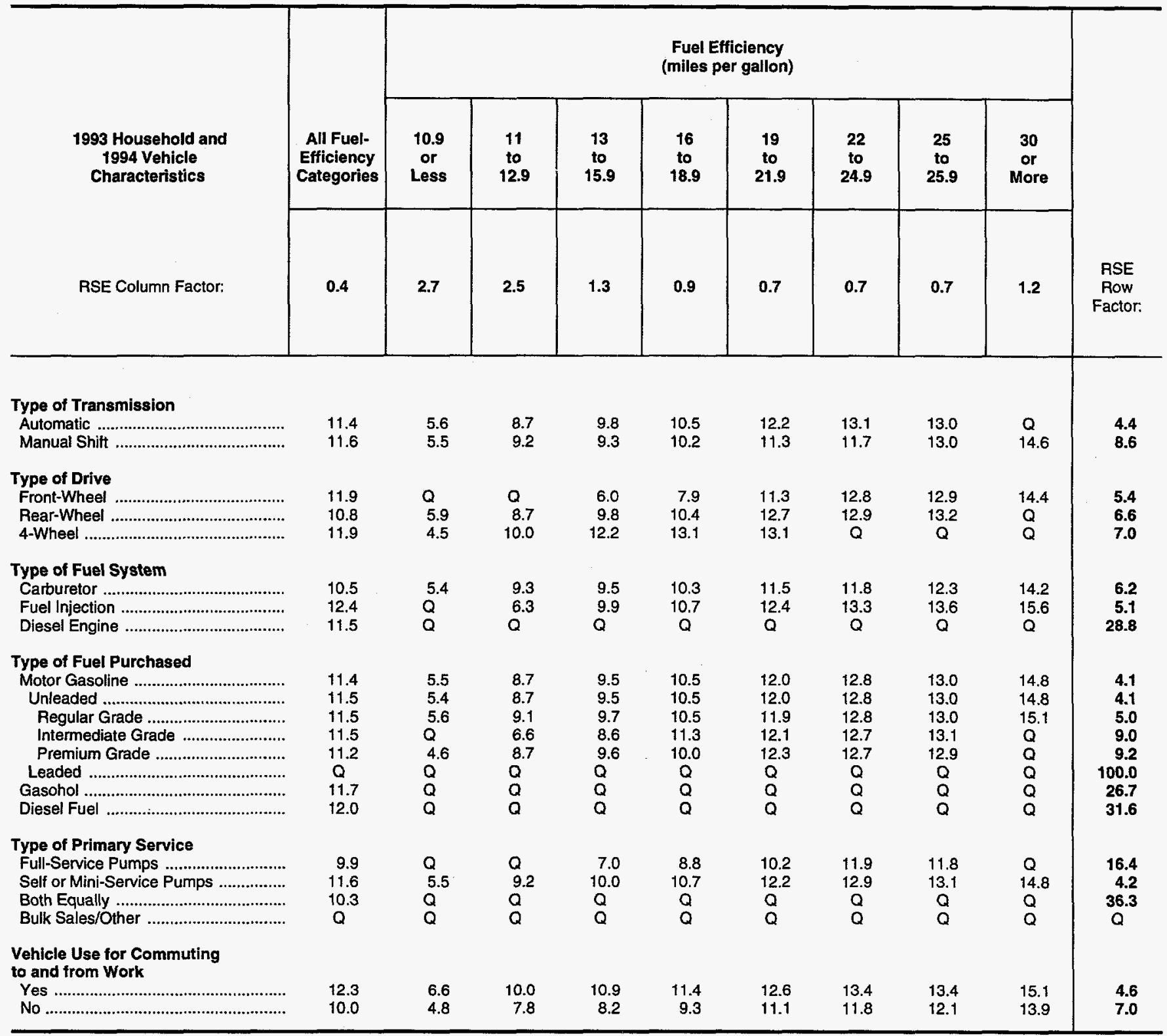

1 Below 150 percent of poverty line or 60 percent of median State income.

2 Approximately .5 percent of the vehicle stock was owned by households that had no drivers as of fall 1993.

NF = No applicable RSE row factor.

$Q=$ Data withheld either because the Relative Standard Error (RSE) was greater than 50 percent or fewer than 10 households were sampled.

Notes: "Households with Children" category includesmembers under age 18 unless the member is the householder or spouse.- To obtain the RSE percentage for any table cell, multiply the corresponding column and row factors. Because of rounding, data may not sum to totals. Data in this table are for households with vehicles for personal use. - Percentages are calculated on unrounded numbers. - See Glossary for definition of terms used in this report.

Source: Energy Information Administration, Office of Energy Markets and End Use, Form ElA-457 A of the 1993 Residential Energy Consumption Survey and Forms EIA-876 A, B, C, and D for the 1994 Residential Transportation Energy Consumption Survey. 
Table 5.14. U.S. Vehicle Fuel Consumption by Vehicle Type, 1994 (Billion Gallons)

\begin{tabular}{|c|c|c|c|c|c|c|c|c|c|c|}
\hline \multirow{3}{*}{$\begin{array}{c}1993 \text { Household and } \\
1994 \text { Vehicle } \\
\text { Characteristics }\end{array}$} & \multirow{3}{*}{$\begin{array}{c}\text { All } \\
\text { Vehicle } \\
\text { Types }\end{array}$} & \multicolumn{8}{|c|}{ Type of Vehicle } & \multirow[b]{4}{*}{$\begin{array}{c}\text { RSE } \\
\text { Aow } \\
\text { Factor: }\end{array}$} \\
\hline & & \multicolumn{3}{|c|}{ Passenger Cars } & \multirow[b]{2}{*}{$\begin{array}{c}\text { Mini- } \\
\text { van }\end{array}$} & \multirow[b]{2}{*}{$\begin{array}{c}\text { Sport } \\
\text { Utility }\end{array}$} & \multirow[b]{2}{*}{$\begin{array}{c}\text { Large } \\
\text { Van }\end{array}$} & \multirow[b]{2}{*}{$\begin{array}{l}\text { Pickup } \\
\text { Truck }\end{array}$} & \multirow[b]{2}{*}{ Other } & \\
\hline & & All & Sedan & $\begin{array}{l}\text { Station } \\
\text { Wagon }\end{array}$ & & & & & & \\
\hline RSE Column Factor: & 0.4 & 0.5 & 0.5 & 1.4 & 1.2 & 1.2 & 1.9 & 0.8 & 3.9 & \\
\hline \multicolumn{11}{|l|}{ Household Characteristics } \\
\hline Total & 90.6 & 54.7 & 51.3 & 3.4 & 5.5 & 7.4 & 2.9 & 19.6 & 0.4 & 8.0 \\
\hline \multicolumn{11}{|l|}{ Census Region and Division } \\
\hline 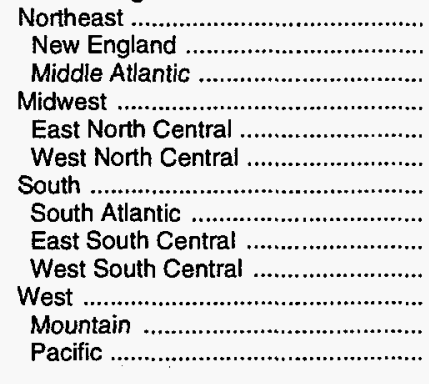 & $\begin{array}{r}14.5 \\
4.1 \\
10.4 \\
23.8 \\
16.7 \\
7.2 \\
33.5 \\
17.1 \\
6.3 \\
10.1 \\
18.8 \\
5.9 \\
12.9\end{array}$ & $\begin{array}{r}9.7 \\
2.5 \\
7.2 \\
15.1 \\
10.5 \\
4.6 \\
19.5 \\
10.5 \\
3.6 \\
5.4 \\
10.4 \\
2.7 \\
7.7\end{array}$ & $\begin{array}{r}8.7 \\
2.2 \\
6.5 \\
14.3 \\
10.0 \\
4.3 \\
18.6 \\
9.9 \\
3.5 \\
5.2 \\
9.7 \\
2.5 \\
7.2\end{array}$ & $\begin{array}{l}1.0 \\
0.3 \\
0.7 \\
0.8 \\
0.5 \\
0.3 \\
0.9 \\
0.6 \\
Q \\
Q \\
0.7 \\
Q \\
0.5\end{array}$ & $\begin{array}{l}0.8 \\
Q \\
0.6 \\
1.8 \\
1.3 \\
0.4 \\
1.9 \\
1.0 \\
0.3 \\
0.6 \\
1.0 \\
Q \\
0.7\end{array}$ & $\begin{array}{l}1.3 \\
0.4 \\
0.9 \\
1.0 \\
0.7 \\
Q \\
3.0 \\
1.7 \\
0.4 \\
0.9 \\
2.0 \\
0.7 \\
1.3\end{array}$ & $\begin{array}{l}Q \\
Q \\
Q \\
1.2 \\
1.0 \\
Q \\
0.9 \\
0.4 \\
Q \\
Q \\
0.5 \\
Q \\
Q\end{array}$ & $\begin{array}{l}2.3 \\
0.9 \\
1.5 \\
4.8 \\
3.2 \\
1.6 \\
8.0 \\
3.4 \\
1.7 \\
2.8 \\
4.6 \\
1.7 \\
2.9\end{array}$ & $\begin{array}{l}Q \\
Q \\
Q \\
Q \\
Q \\
Q \\
Q \\
Q \\
Q \\
Q \\
Q \\
Q \\
Q\end{array}$ & $\begin{array}{l}16.2 \\
29.7 \\
18.5 \\
13.4 \\
16.7 \\
20.1 \\
13.0 \\
16.0 \\
12.3 \\
31.9 \\
17.8 \\
24.7 \\
23.4\end{array}$ \\
\hline $\begin{array}{l}\text { Largest Populated States } \\
\text { California } \\
\text { Florida } \\
\text { New York } \\
\text { Texas }\end{array}$ & $\begin{array}{l}9.5 \\
5.1 \\
4.3 \\
6.6\end{array}$ & $\begin{array}{l}5.7 \\
3.2 \\
3.1 \\
3.6\end{array}$ & $\begin{array}{l}5.3 \\
2.9 \\
2.7 \\
3.4\end{array}$ & $\begin{array}{l}0.4 \\
Q \\
0.3 \\
Q\end{array}$ & $\begin{array}{l}0.6 \\
0.3 \\
Q \\
Q\end{array}$ & $\begin{array}{l}0.9 \\
0.6 \\
Q \\
0.7\end{array}$ & $\begin{array}{l}Q \\
Q \\
Q \\
Q\end{array}$ & $\begin{array}{l}2.1 \\
0.9 \\
0.5 \\
1.7\end{array}$ & $\begin{array}{l}Q \\
Q \\
Q \\
Q\end{array}$ & $\begin{array}{l}28.8 \\
18.8 \\
23.8 \\
31.3\end{array}$ \\
\hline 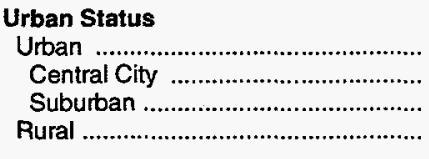 & $\begin{array}{l}67.5 \\
21.4 \\
46.1 \\
23.1\end{array}$ & $\begin{array}{l}42.6 \\
14.1 \\
28.5 \\
12.1\end{array}$ & $\begin{array}{l}39.8 \\
13.1 \\
26.6 \\
11.5\end{array}$ & $\begin{array}{l}2.8 \\
0.9 \\
1.9 \\
0.6\end{array}$ & $\begin{array}{l}4.4 \\
1.3 \\
3.1 \\
1.1\end{array}$ & $\begin{array}{l}5.8 \\
1.7 \\
4.1 \\
1.6\end{array}$ & $\begin{array}{l}2.3 \\
0.8 \\
1.5 \\
0.5\end{array}$ & $\begin{array}{r}12.1 \\
3.3 \\
8.8 \\
7.5\end{array}$ & $\begin{array}{l}Q \\
Q \\
Q \\
Q\end{array}$ & $\begin{array}{r}9.2 \\
18.8 \\
11.6 \\
15.0\end{array}$ \\
\hline 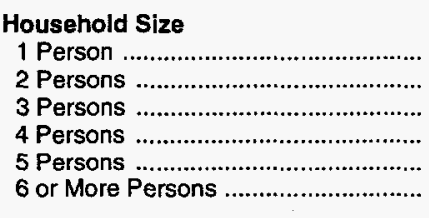 & $\begin{array}{r}9.6 \\
30.6 \\
18.9 \\
18.1 \\
9.1 \\
4.2\end{array}$ & $\begin{array}{r}7.1 \\
19.5 \\
11.4 \\
9.5 \\
4.8 \\
2.5\end{array}$ & $\begin{array}{r}6.7 \\
18.7 \\
10.5 \\
8.8 \\
4.2 \\
2.3\end{array}$ & $\begin{array}{l}0.3 \\
0.9 \\
0.9 \\
0.6 \\
0.5 \\
Q\end{array}$ & $\begin{array}{l}Q \\
0.8 \\
0.9 \\
1.9 \\
1.3 \\
0.5\end{array}$ & $\begin{array}{l}0.5 \\
2.2 \\
1.8 \\
1.7 \\
1.0 \\
Q\end{array}$ & $\begin{array}{l}Q \\
0.7 \\
0.5 \\
0.8 \\
Q \\
Q\end{array}$ & $\begin{array}{l}1.6 \\
7.1 \\
4.2 \\
4.3 \\
1.5 \\
0.8\end{array}$ & $\begin{array}{l}Q \\
Q \\
Q \\
Q \\
Q \\
Q\end{array}$ & $\begin{array}{l}21.7 \\
12.9 \\
17.1 \\
18.1 \\
25.9 \\
42.8\end{array}$ \\
\hline $\begin{array}{l}\text { Household Composition } \\
\text { Households with Children ................ } \\
\text { Age of Oldest Child }\end{array}$ & 40.4 & 22.1 & 20.3 & 1.8 & 4.3 & 3.8 & 1.5 & 8.5 & Q & 12.0 \\
\hline 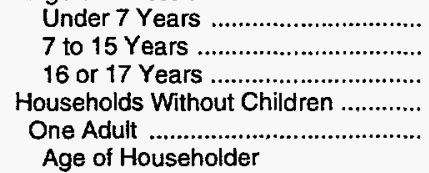 & $\begin{array}{r}10.3 \\
20.5 \\
9.6 \\
50.2 \\
9.6\end{array}$ & $\begin{array}{r}6.0 \\
10.5 \\
5.7 \\
32.6 \\
7.1\end{array}$ & $\begin{array}{r}5.5 \\
9.5 \\
5.3 \\
31.0 \\
6.7\end{array}$ & $\begin{array}{l}0.4 \\
1.0 \\
0.4 \\
1.6 \\
0.3\end{array}$ & $\begin{array}{l}0.8 \\
2.7 \\
0.8 \\
1.2 \\
Q\end{array}$ & $\begin{array}{l}1.2 \\
2.0 \\
0.6 \\
3.6 \\
0.5\end{array}$ & $\begin{array}{l}Q \\
0.9 \\
Q \\
1.4 \\
Q\end{array}$ & $\begin{array}{r}2.2 \\
4.3 \\
2.1 \\
11.1 \\
1.6\end{array}$ & $\begin{array}{l}Q \\
Q \\
Q \\
Q \\
Q\end{array}$ & $\begin{array}{l}22.5 \\
15.7 \\
26.2 \\
10.6 \\
21.7\end{array}$ \\
\hline $\begin{array}{l}\text { Under } 35 \text { Years .......................... } \\
35 \text { to } 59 \text { Years ............................ } \\
60 \text { Years or More ...................... }\end{array}$ & $\begin{array}{l}2.4 \\
4.1 \\
3.0\end{array}$ & $\begin{array}{l}1.6 \\
2.8 \\
2.6\end{array}$ & $\begin{array}{l}1.6 \\
2.6 \\
2.5\end{array}$ & $\begin{array}{l}Q \\
Q \\
Q\end{array}$ & $\begin{array}{l}Q \\
Q \\
Q\end{array}$ & $\begin{array}{l}Q \\
Q \\
Q\end{array}$ & $\begin{array}{l}Q \\
Q \\
Q\end{array}$ & $\begin{array}{l}0.5 \\
0.8 \\
0.3\end{array}$ & $\begin{array}{l}Q \\
Q \\
Q\end{array}$ & $\begin{array}{l}40.5 \\
32.5 \\
27.4\end{array}$ \\
\hline
\end{tabular}

See footnotes at end of table. 
Table 5.14. U.S. Vehicle Fuel Consumption by Vehicle Type, 1994 (Continued) (Billion Gallons)

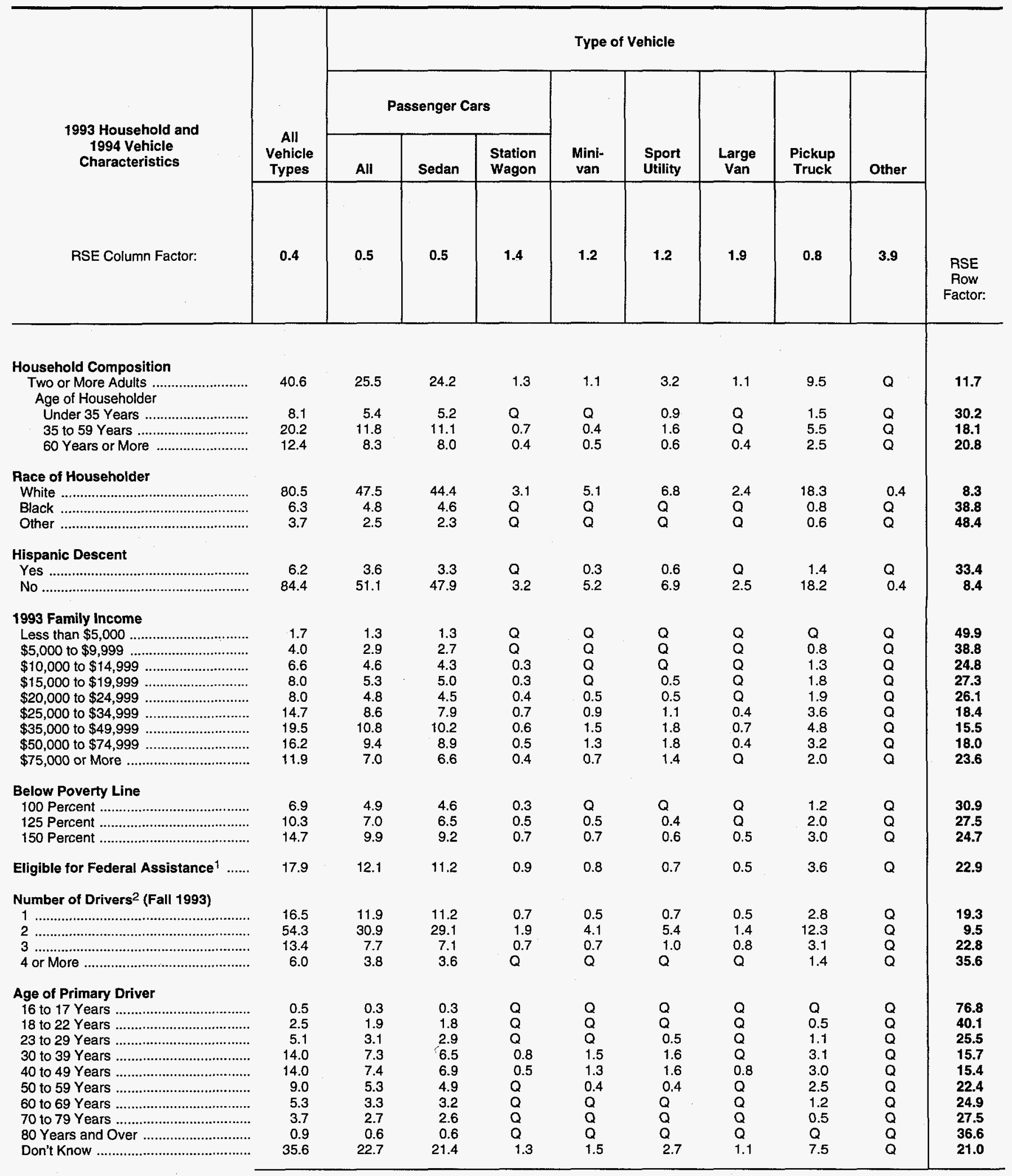

See footnotes at end of table. 
Table 5.14. U.S. Vehicle Fuel Consumption by Vehicle Type, 1994 (Continued) (Billion Gallons)

\begin{tabular}{|c|c|c|c|c|c|c|c|c|c|c|}
\hline \multirow{4}{*}{$\begin{array}{l}1993 \text { Household and } \\
1994 \text { Vehicle } \\
\text { Characteristics } \\
\text { RSE Column Factor: }\end{array}$} & \multirow{3}{*}{$\begin{array}{l}\text { All } \\
\text { Vehicle } \\
\text { Types }\end{array}$} & \multicolumn{8}{|c|}{ Type of Vehicle } & \multirow[b]{4}{*}{$\begin{array}{l}\text { RSE } \\
\text { Row } \\
\text { Factor: }\end{array}$} \\
\hline & & \multicolumn{3}{|c|}{ Passenger Cars } & \multirow[b]{2}{*}{$\begin{array}{c}\text { Mini- } \\
\text { van }\end{array}$} & \multirow[b]{2}{*}{$\begin{array}{r}\text { Sport } \\
\text { Utility }\end{array}$} & \multirow[b]{2}{*}{$\begin{array}{l}\text { Large } \\
\text { Van }\end{array}$} & \multirow[b]{2}{*}{$\begin{array}{l}\text { Pickup } \\
\text { Truck }\end{array}$} & \multirow[b]{2}{*}{ Other } & \\
\hline & & All & Sedan & $\begin{array}{l}\text { Station } \\
\text { Wagon }\end{array}$ & & & & & & \\
\hline & 0.4 & 0.5 & 0.5 & 1.4 & 1.2 & 1.2 & 1.9 & 0.8 & 3.9 & \\
\hline \multicolumn{11}{|l|}{ Sex of Primary Driver } \\
\hline 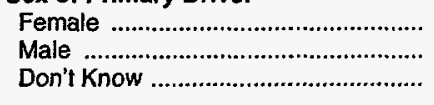 & $\begin{array}{l}23.5 \\
31.5 \\
35.6\end{array}$ & $\begin{array}{l}17.0 \\
15.0 \\
22.6\end{array}$ & $\begin{array}{l}16.0 \\
14.0 \\
21.3\end{array}$ & $\begin{array}{l}1.1 \\
1.1 \\
1.3\end{array}$ & $\begin{array}{l}2.9 \\
1.1 \\
1.5\end{array}$ & $\begin{array}{l}1.8 \\
3.0 \\
2.7\end{array}$ & $\begin{array}{l}0.6 \\
1.2 \\
1.1\end{array}$ & $\begin{array}{r}1.2 \\
10.9 \\
7.5\end{array}$ & $\begin{array}{l}Q \\
Q\end{array}$ & $\begin{array}{l}11.7 \\
11.9 \\
20.9\end{array}$ \\
\hline \multicolumn{11}{|l|}{$\begin{array}{l}\text { Average Number of Vehicles per } \\
\text { Household During the Year }\end{array}$} \\
\hline $\begin{array}{l}\text { Part-Year Vehicle } \\
\text { Only } 1 \\
\text { Between } 1 \text { and } 2 \\
\text { Only } 2 \\
\text { Between } 2 \text { and } 3 \\
\text { Only } 3 \\
\text { Between } 3 \text { and } 4 \\
4 \text { or More }\end{array}$ & $\begin{array}{r}0.7 \\
14.8 \\
5.4 \\
32.9 \\
8.5 \\
14.5 \\
4.9 \\
8.7\end{array}$ & $\begin{array}{r}0.6 \\
11.2 \\
3.4 \\
18.8 \\
4.7 \\
8.0 \\
2.9 \\
5.1\end{array}$ & $\begin{array}{r}0.6 \\
10.5 \\
3.2 \\
17.6 \\
4.5 \\
7.4 \\
2.7 \\
4.8\end{array}$ & $\begin{array}{l}Q \\
0.6 \\
0.2 \\
1.2 \\
0.2 \\
0.6 \\
Q \\
0.3\end{array}$ & $\begin{array}{l}Q \\
0.7 \\
0.4 \\
2.5 \\
0.6 \\
0.7 \\
Q \\
0.4\end{array}$ & $\begin{array}{l}Q \\
0.7 \\
0.4 \\
3.2 \\
1.1 \\
1.0 \\
0.4 \\
0.6\end{array}$ & $\begin{array}{l}Q \\
Q \\
Q \\
1.2 \\
Q \\
0.6 \\
Q \\
Q\end{array}$ & $\begin{array}{l}Q \\
1.9 \\
1.0 \\
7.2 \\
1.9 \\
4.0 \\
1.3 \\
2.2\end{array}$ & $\begin{array}{l}Q \\
Q \\
Q \\
Q \\
Q \\
Q \\
Q \\
Q\end{array}$ & $\begin{array}{r}102.4 \\
19.0 \\
32.1 \\
12.7 \\
22.2 \\
21.2 \\
37.4 \\
27.3\end{array}$ \\
\hline \multicolumn{11}{|l|}{ Vehicle Characteristics } \\
\hline 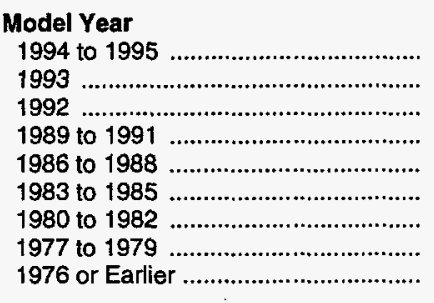 & $\begin{array}{r}5.0 \\
6.6 \\
6.9 \\
20.7 \\
19.0 \\
13.3 \\
5.6 \\
6.3 \\
7.2\end{array}$ & $\begin{array}{r}2.4 \\
3.8 \\
4.3 \\
11.8 \\
12.4 \\
8.7 \\
4.0 \\
3.6 \\
3.8\end{array}$ & $\begin{array}{r}2.3 \\
3.5 \\
4.1 \\
11.4 \\
11.7 \\
7.7 \\
3.6 \\
3.4 \\
3.6\end{array}$ & $\begin{array}{l}Q \\
0.3 \\
Q \\
0.4 \\
0.8 \\
1.0 \\
0.4 \\
Q \\
Q\end{array}$ & $\begin{array}{l}0.7 \\
0.8 \\
0.7 \\
2.0 \\
1.1 \\
0.3 \\
Q \\
Q \\
Q\end{array}$ & $\begin{array}{l}0.6 \\
0.8 \\
0.8 \\
2.1 \\
1.6 \\
0.8 \\
Q \\
Q \\
Q\end{array}$ & $\begin{array}{l}Q \\
Q \\
Q \\
0.7 \\
Q \\
0.7 \\
Q \\
Q \\
Q\end{array}$ & $\begin{array}{l}1.3 \\
1.1 \\
1.1 \\
4.0 \\
3.3 \\
2.8 \\
1.1 \\
2.2 \\
2.6\end{array}$ & $\begin{array}{l}Q \\
Q \\
Q \\
Q \\
Q \\
Q \\
Q \\
Q \\
Q\end{array}$ & $\begin{array}{l}25.3 \\
22.0 \\
21.5 \\
14.5 \\
15.1 \\
19.7 \\
29.8 \\
38.0 \\
34.9\end{array}$ \\
\hline $\begin{array}{l}\text { Fuel Efficiency (miles per gallon) } \\
10.9 \text { or Less } \\
11 \text { to } 12.9 \\
13 \text { to } 15.9 \\
16 \text { to } 18.9 \\
19 \text { to } 21.9 \\
22 \text { to } 24.9 \\
25 \text { to } 29.9\end{array}$ & $\begin{array}{r}4.1 \\
6.5 \\
12.6 \\
15.9 \\
20.8 \\
15.5 \\
12.0 \\
3.2\end{array}$ & $\begin{array}{r}1.0 \\
2.3 \\
3.8 \\
6.8 \\
13.2 \\
13.3 \\
11.3 \\
3.1\end{array}$ & $\begin{array}{r}0.9 \\
2.1 \\
3.6 \\
6.2 \\
12.5 \\
12.4 \\
10.6 \\
2.9\end{array}$ & $\begin{array}{l}Q \\
Q \\
Q \\
0.5 \\
0.7 \\
0.9 \\
0.7 \\
Q\end{array}$ & $\begin{array}{l}Q \\
Q \\
Q \\
1.7 \\
3.3 \\
0.4 \\
Q \\
Q\end{array}$ & $\begin{array}{l}0.3 \\
0.9 \\
1.5 \\
3.2 \\
1.3 \\
Q \\
Q \\
Q\end{array}$ & $\begin{array}{l}Q \\
Q \\
1.5 \\
Q \\
Q \\
Q \\
Q \\
Q\end{array}$ & $\begin{array}{l}1.9 \\
2.9 \\
5.7 \\
3.8 \\
2.9 \\
1.7 \\
0.7 \\
Q\end{array}$ & $\begin{array}{l}0.4 \\
Q \\
Q \\
Q \\
Q \\
Q \\
Q \\
Q\end{array}$ & $\begin{array}{l}25.7 \\
34.8 \\
20.9 \\
16.0 \\
15.2 \\
17.2 \\
16.1 \\
33.0\end{array}$ \\
\hline 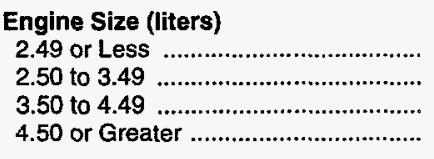 & $\begin{array}{l}27.4 \\
17.5 \\
15.1 \\
30.6\end{array}$ & $\begin{array}{r}22.9 \\
10.4 \\
8.1 \\
13.2\end{array}$ & $\begin{array}{r}21.2 \\
9.8 \\
7.8 \\
12.5\end{array}$ & $\begin{array}{l}1.7 \\
0.6 \\
0.4 \\
0.7\end{array}$ & $\begin{array}{l}0.7 \\
3.3 \\
1.5 \\
Q\end{array}$ & $\begin{array}{l}0.4 \\
1.6 \\
3.1 \\
2.3\end{array}$ & $\begin{array}{l}Q \\
Q \\
Q \\
2.5\end{array}$ & $\begin{array}{r}3.4 \\
2.0 \\
2.0 \\
12.1\end{array}$ & $\begin{array}{l}Q \\
Q \\
Q \\
0.4\end{array}$ & $\begin{array}{l}14.8 \\
13.5 \\
18.2 \\
14.5\end{array}$ \\
\hline $\begin{array}{l}\text { Number of Cylinders } \\
4 \\
6\end{array}$ & $\begin{array}{r}28.3 \\
32.3 \\
29.4 \\
0.5\end{array}$ & $\begin{array}{r}22.8 \\
17.4 \\
14.0 \\
0.5\end{array}$ & $\begin{array}{r}21.1 \\
16.4 \\
13.3 \\
0.5\end{array}$ & $\begin{array}{l}1.7 \\
1.0 \\
0.7 \\
Q\end{array}$ & $\begin{array}{l}1.1 \\
4.4 \\
Q \\
Q\end{array}$ & $\begin{array}{l}0.6 \\
4.6 \\
2.2 \\
Q\end{array}$ & $\begin{array}{l}Q \\
0.6 \\
2.3 \\
Q\end{array}$ & $\begin{array}{c}3.8 \\
5.3 \\
10.5 \\
0\end{array}$ & $\begin{array}{l}Q \\
Q \\
0.4 \\
Q\end{array}$ & $\begin{array}{l}12.9 \\
12.4 \\
14.4 \\
60.1\end{array}$ \\
\hline
\end{tabular}

See footnotes at end of table. 
Table 5.14. U.S. Vehicle Fuel Consumption by Vehicle Type, 1994 (Continued) (Billion Gallons)

\begin{tabular}{|c|c|c|c|c|c|c|c|c|c|c|}
\hline \multirow{3}{*}{$\begin{array}{l}1993 \text { Household and } \\
1994 \text { Vehicle } \\
\text { Characteristics }\end{array}$} & \multirow{3}{*}{$\begin{array}{l}\text { All } \\
\text { Vehicle } \\
\text { Types }\end{array}$} & \multicolumn{8}{|c|}{ Type of Vehicle } & \multirow[b]{4}{*}{$\begin{array}{l}\text { RSE } \\
\text { Row } \\
\text { Factor: }\end{array}$} \\
\hline & & \multicolumn{3}{|c|}{ Passenger Cars } & \multirow[b]{2}{*}{$\begin{array}{c}\text { Mini- } \\
\text { van }\end{array}$} & \multirow[b]{2}{*}{$\begin{array}{l}\text { Sport } \\
\text { Utility }\end{array}$} & \multirow[b]{2}{*}{$\begin{array}{c}\text { Large } \\
\text { Van }\end{array}$} & \multirow[b]{2}{*}{$\begin{array}{c}\text { Pickup } \\
\text { Truck }\end{array}$} & \multirow[b]{2}{*}{ Other } & \\
\hline & & All & Sedan & $\begin{array}{l}\text { Station } \\
\text { Wagon }\end{array}$ & & & & & & \\
\hline RSE Column Factor: & 0.4 & 0.5 & 0.5 & 1.4 & 1.2 & 1.2 & 1.9 & 0.8 & 3.9 & \\
\hline \multicolumn{11}{|l|}{ Type of Transmission } \\
\hline 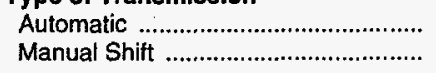 & $\begin{array}{l}69.8 \\
20.7\end{array}$ & $\begin{array}{r}45.0 \\
9.7\end{array}$ & $\begin{array}{r}42.4 \\
8.9\end{array}$ & $\begin{array}{l}2.6 \\
0.8\end{array}$ & $\begin{array}{l}5.1 \\
0.4\end{array}$ & $\begin{array}{l}5.8 \\
1.7\end{array}$ & $\begin{array}{l}2.7 \\
Q\end{array}$ & $\begin{array}{r}10.8 \\
8.8\end{array}$ & $Q^{0.4}$ & $\begin{array}{r}8.9 \\
16.7\end{array}$ \\
\hline $\begin{array}{l}\text { Type of Drive } \\
\text { Front-Wheel } \\
\text { Rear-Wheel }\end{array}$ & $\begin{array}{l}38.1 \\
39.7 \\
12.7\end{array}$ & $\begin{array}{r}32.7 \\
21.2 \\
0.9\end{array}$ & $\begin{array}{r}30.9 \\
19.8 \\
0.5\end{array}$ & $\begin{array}{l}1.8 \\
1.3 \\
0.3\end{array}$ & $\begin{array}{l}3.4 \\
1.6 \\
Q\end{array}$ & $\begin{array}{l}Q \\
1.6 \\
5.4\end{array}$ & $\begin{array}{l}Q \\
2.7 \\
Q\end{array}$ & $\begin{array}{r}1.4 \\
12.3 \\
5.9\end{array}$ & $\begin{array}{l}Q \\
0.3 \\
Q\end{array}$ & $\begin{array}{l}10.6 \\
12.9 \\
24.5\end{array}$ \\
\hline $\begin{array}{l}\text { Type of Fuel System } \\
\text { Carburetor } \\
\text { Fuel Injection } \\
\text { Diesel Engine }\end{array}$ & $\begin{array}{r}43.9 \\
45.3 \\
1.4\end{array}$ & $\begin{array}{r}25.7 \\
28.6 \\
0.4\end{array}$ & $\begin{array}{r}23.4 \\
27.4 \\
0.4\end{array}$ & $\begin{array}{l}2.3 \\
1.2 \\
Q\end{array}$ & $\begin{array}{l}0.9 \\
4.7 \\
Q\end{array}$ & $\begin{array}{l}2.7 \\
4.7 \\
Q\end{array}$ & $\begin{array}{l}2.1 \\
0.8 \\
Q\end{array}$ & $\begin{array}{r}12.3 \\
6.4 \\
0.9\end{array}$ & $\begin{array}{l}Q \\
Q \\
Q\end{array}$ & $\begin{array}{l}13.0 \\
10.4 \\
54.8\end{array}$ \\
\hline 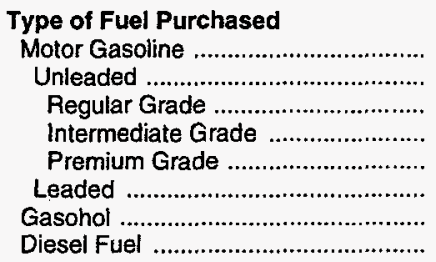 & $\begin{array}{r}88.3 \\
87.0 \\
59.9 \\
11.7 \\
15.4 \\
1.2 \\
0.9 \\
1.2\end{array}$ & $\begin{array}{c}53.8 \\
53.1 \\
35.7 \\
7.2 \\
10.2 \\
Q \\
0.6 \\
0.3\end{array}$ & $\begin{array}{l}50.4 \\
49.7 \\
33.4 \\
6.7 \\
9.7 \\
Q \\
0.6 \\
0.3\end{array}$ & $\begin{array}{l}3.4 \\
3.4 \\
2.4 \\
0.5 \\
Q \\
Q \\
Q \\
Q\end{array}$ & $\begin{array}{l}5.5 \\
5.5 \\
3.9 \\
0.7 \\
0.8 \\
Q \\
Q \\
Q\end{array}$ & $\begin{array}{l}7.3 \\
7.3 \\
4.7 \\
1.3 \\
1.4 \\
Q \\
Q \\
Q\end{array}$ & $\begin{array}{l}2.9 \\
2.9 \\
2.1 \\
Q \\
Q \\
Q \\
Q \\
Q\end{array}$ & $\begin{array}{c}18.4 \\
17.9 \\
13.2 \\
2.1 \\
2.6 \\
Q \\
Q \\
0.9\end{array}$ & $\begin{array}{l}0.4 \\
0.4 \\
Q \\
Q \\
Q \\
Q \\
Q \\
Q\end{array}$ & $\begin{array}{r}8.1 \\
8.1 \\
9.3 \\
18.7 \\
18.4 \\
97.7 \\
63.8 \\
59.3\end{array}$ \\
\hline 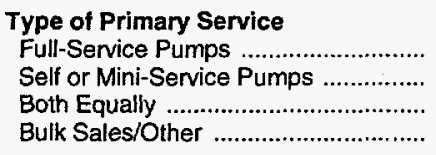 & $\begin{array}{r}6.8 \\
82.0 \\
1.6 \\
Q\end{array}$ & $\begin{array}{r}5.0 \\
48.7 \\
1.0 \\
a\end{array}$ & $\begin{array}{c}4.7 \\
45.6 \\
0.9 \\
Q\end{array}$ & $\begin{array}{l}0.3 \\
3.0 \\
Q \\
Q\end{array}$ & $\begin{array}{l}0.4 \\
5.1 \\
0 \\
0\end{array}$ & $\begin{array}{l}0.5 \\
6.7 \\
Q \\
Q\end{array}$ & $\begin{array}{l}Q \\
2.7 \\
Q \\
Q\end{array}$ & $\begin{array}{c}0.8 \\
18.4 \\
Q \\
Q\end{array}$ & $\begin{array}{l}Q \\
0.4 \\
Q \\
Q\end{array}$ & $\begin{array}{r}35.2 \\
8.4 \\
62.3 \\
Q\end{array}$ \\
\hline \multicolumn{10}{|l|}{$\begin{array}{l}\text { Vehicle Use for Commuting } \\
\text { to and from Work }\end{array}$} & $\begin{array}{r}9.5 \\
12.6\end{array}$ \\
\hline
\end{tabular}

1 Below 150 percent of poverty line or 60 percent of median State income.

2 Approximately .5 percent of the vehicle stock was owned by households that had no drivers as of fall 1993

NF $=$ No applicable RSE row factor.

$Q=$ Data withheld either because the Relative Standard Error (RSE) was greater than 50 percent or fewer than 10 households were sampled.

Notes: "Households with Children" category includes members under age 18 unless the member is the householder or spouse.* To obtain the RSE percentage for any table cell, multiply the corresponding column and row factors. - Because of rounding, data may not sum to totals. - Data in this table are for households with vehicles for personal use. - Percentages are calculated on unrounded numbers. - See Glossary for definition of terms used in this report.

Source: Energy Information Administration, Office of Energy Markets and End Use, Form ElA-457 A of the 1993 Residential Energy Consumption Survey and Forms ElA-876 A, B, C, and D for the 1994 Residential Transportation Energy Consumption Survey. 
Table 5.15. U.S. Average Vehicle-Miles Traveled by Vehicle Type, 1994

(Thousand Miles per Vehicle)

\begin{tabular}{|c|c|c|c|c|c|c|c|c|c|c|}
\hline \multirow{3}{*}{$\begin{array}{c}1993 \text { Househoid and } \\
1994 \text { Vehicle } \\
\text { Characteristics }\end{array}$} & \multirow{3}{*}{$\begin{array}{l}\text { All } \\
\text { Vehicle } \\
\text { Types }\end{array}$} & \multicolumn{8}{|c|}{ Type of Vehicle } & \multirow[b]{4}{*}{$\begin{array}{l}\text { RSE } \\
\text { Row } \\
\text { Factor: }\end{array}$} \\
\hline & & \multicolumn{3}{|c|}{ Passenger Cars } & \multirow[b]{2}{*}{$\begin{array}{c}\text { Mini- } \\
\text { van }\end{array}$} & \multirow[b]{2}{*}{$\begin{array}{l}\text { Sport } \\
\text { Utility }\end{array}$} & \multirow[b]{2}{*}{$\begin{array}{l}\text { Large } \\
\text { Van }\end{array}$} & \multirow[b]{2}{*}{$\begin{array}{l}\text { Pickup } \\
\text { Truck }\end{array}$} & \multirow[b]{2}{*}{ Other } & \\
\hline & & All & Sedan & $\begin{array}{l}\text { Station } \\
\text { Wagon }\end{array}$ & & & & & & \\
\hline RSE Column Factor: & 0.3 & 0.4 & 0.4 & $1: 4$ & 0.9 & 1.2 & 2.4 & 0.8 & 5.4 & \\
\hline Household Characteristics & & & & & & & & & & \\
\hline Total & 11.4 & 11.3 & 11.2 & 12.1 & 13.4 & 12.7 & 11.7 & 11.1 & 7.1 & 4.2 \\
\hline Census Region and Division & & & & & & & & & & \\
\hline 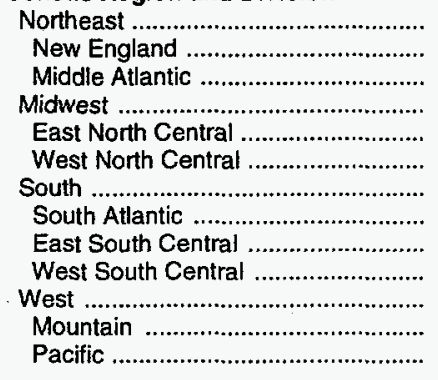 & $\begin{array}{l}11.3 \\
11.1 \\
11.3 \\
11.6 \\
11.6 \\
11.8 \\
11.7 \\
12.1 \\
10.9 \\
11.6 \\
10.9 \\
10.7 \\
10.9\end{array}$ & $\begin{array}{l}11.0 \\
10.5 \\
11.2 \\
11.6 \\
11.5 \\
12.0 \\
11.4 \\
11.9 \\
10.7 \\
11.0 \\
10.8 \\
10.5 \\
10.9\end{array}$ & $\begin{array}{l}10.9 \\
10.4 \\
11.1 \\
11.6 \\
11.5 \\
11.9 \\
11.4 \\
11.8 \\
10.7 \\
11.0 \\
10.7 \\
10.3 \\
10.9\end{array}$ & $\begin{array}{l}12.2 \\
11.1 \\
12.9 \\
11.6 \\
10.8 \\
13.0 \\
12.5 \\
13.6 \\
Q \\
Q \\
12.0 \\
Q \\
11.6\end{array}$ & $\begin{array}{c}12.5 \\
Q \\
12.5 \\
14.0 \\
13.9 \\
14.5 \\
13.8 \\
12.7 \\
14.4 \\
15.6 \\
12.6 \\
Q \\
12.4\end{array}$ & $\begin{array}{c}12.5 \\
12.0 \\
12.7 \\
11.0 \\
10.7 \\
Q \\
14.7 \\
15.0 \\
13.9 \\
14.6 \\
11.3 \\
11.0 \\
11.5\end{array}$ & $\begin{array}{c}Q \\
Q \\
Q \\
13.1 \\
13.1 \\
Q \\
11.4 \\
10.6 \\
Q \\
Q \\
9.4 \\
Q \\
Q\end{array}$ & $\begin{array}{l}11.4 \\
12.3 \\
10.9 \\
10.8 \\
10.9 \\
10.6 \\
11.4 \\
12.1 \\
10.2 \\
11.4 \\
10.8 \\
10.7 \\
10.9\end{array}$ & $\begin{array}{l}Q \\
Q \\
Q \\
Q \\
Q \\
Q \\
Q \\
Q \\
Q \\
Q \\
Q \\
Q \\
Q\end{array}$ & $\begin{array}{r}8.1 \\
10.3 \\
10.0 \\
6.2 \\
7.1 \\
9.5 \\
7.5 \\
10.1 \\
20.9 \\
14.1 \\
11.0 \\
19.4 \\
12.6\end{array}$ \\
\hline $\begin{array}{l}\text { Largest Populated States } \\
\text { California } \\
\text { Florida } \\
\text { New York } \\
\text { Texas }\end{array}$ & $\begin{array}{l}11.1 \\
11.8 \\
11.4 \\
11.3\end{array}$ & $\begin{array}{l}11.1 \\
11.0 \\
11.4 \\
10.6\end{array}$ & $\begin{array}{l}11.0 \\
10.9 \\
11.2 \\
10.7\end{array}$ & $\begin{array}{l}13.0 \\
Q \\
13.4 \\
Q\end{array}$ & $\begin{array}{c}12.6 \\
12.2 \\
Q \\
Q\end{array}$ & $\begin{array}{l}11.2 \\
15.5 \\
Q \\
14.6\end{array}$ & $\begin{array}{l}Q \\
Q \\
Q \\
Q\end{array}$ & $\begin{array}{l}11.5 \\
13.8 \\
10.7 \\
11.0\end{array}$ & $\begin{array}{l}Q \\
Q \\
Q \\
Q\end{array}$ & $\begin{array}{l}15.3 \\
13.3 \\
11.6 \\
11.7\end{array}$ \\
\hline 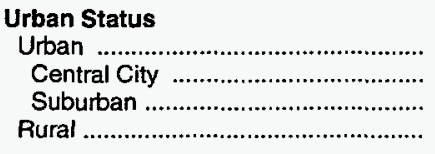 & $\begin{array}{l}11.4 \\
10.8 \\
11.7 \\
11.6\end{array}$ & $\begin{array}{l}11.2 \\
10.7 \\
11.5 \\
11.5\end{array}$ & $\begin{array}{l}11.1 \\
10.6 \\
11.4 \\
11.6\end{array}$ & $\begin{array}{l}12.3 \\
11.7 \\
12.6 \\
11.1\end{array}$ & $\begin{array}{l}13.2 \\
12.1 \\
13.7 \\
14.3\end{array}$ & $\begin{array}{l}12.5 \\
12.4 \\
12.6 \\
13.3\end{array}$ & $\begin{array}{l}11.5 \\
10.7 \\
12.0 \\
12.3\end{array}$ & $\begin{array}{l}11.2 \\
10.5 \\
11.5 \\
10.9\end{array}$ & $\begin{array}{l}Q \\
Q \\
Q \\
Q\end{array}$ & $\begin{array}{l}4.7 \\
9.3 \\
5.6 \\
7.6\end{array}$ \\
\hline 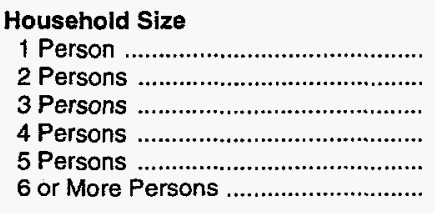 & $\begin{array}{l}10.1 \\
10.9 \\
11.9 \\
12.0 \\
12.2 \\
13.3\end{array}$ & $\begin{array}{r}9.9 \\
10.8 \\
12.0 \\
11.6 \\
12.2 \\
14.1\end{array}$ & $\begin{array}{l}10.0 \\
10.7 \\
11.9 \\
11.7 \\
11.9 \\
14.3\end{array}$ & $\begin{array}{l}9.5 \\
12.7 \\
12.5 \\
11.0 \\
14.6 \\
Q\end{array}$ & $\begin{array}{l}Q \\
12.8 \\
13.0 \\
14.0 \\
13.4 \\
14.4\end{array}$ & $\begin{array}{l}12.0 \\
12.4 \\
13.8 \\
12.3 \\
12.0 \\
a\end{array}$ & $\begin{array}{l}\mathrm{Q} \\
10.1 \\
12.5 \\
11.5 \\
14.4 \\
\mathrm{Q}\end{array}$ & $\begin{array}{l}10.6 \\
10.7 \\
11.0 \\
12.5 \\
11.2 \\
10.1\end{array}$ & $\begin{array}{l}Q \\
Q \\
Q \\
Q \\
Q \\
Q\end{array}$ & $\begin{array}{r}16.2 \\
7.9 \\
9.0 \\
9.7 \\
13.0 \\
18.8\end{array}$ \\
\hline $\begin{array}{l}\text { Household Composition } \\
\text { Households with Children ................. } \\
\text { Age of Oldest Child }\end{array}$ & 12.3 & 12.3 & 12.2 & 12.7 & 13.8 & 12.7 & 12.5 & 11.6 & $Q$ & 6.1 \\
\hline 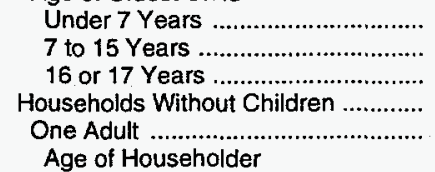 & $\begin{array}{l}12.1 \\
12.3 \\
12.5 \\
10.8 \\
10.1\end{array}$ & $\begin{array}{r}12.0 \\
12.1 \\
12.8 \\
10.7 \\
9.9\end{array}$ & $\begin{array}{l}12.0 \\
12.1 \\
12.7 \\
10.6 \\
10.0\end{array}$ & $\begin{array}{r}12.2 \\
12.9 \\
12.9 \\
11.5 \\
9.5\end{array}$ & $\begin{array}{c}12.7 \\
14.4 \\
13.3 \\
12.1 \\
Q\end{array}$ & $\begin{array}{l}12.7 \\
13.0 \\
11.8 \\
12.7 \\
12.0\end{array}$ & $\begin{array}{l}Q \\
12.3 \\
Q \\
10.8 \\
Q\end{array}$ & $\begin{array}{l}12.0 \\
11.4 \\
11.5 \\
10.8 \\
10.6\end{array}$ & $\begin{array}{l}Q \\
Q \\
Q \\
Q \\
Q\end{array}$ & $\begin{array}{r}12.2 \\
8.4 \\
12.9 \\
6.2 \\
16.2\end{array}$ \\
\hline 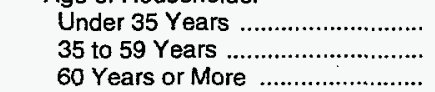 & $\begin{array}{r}13.0 \\
11.2 \\
7.6\end{array}$ & $\begin{array}{r}13.2 \\
11.2 \\
7.6\end{array}$ & $\begin{array}{r}13.2 \\
11.2 \\
7.6\end{array}$ & $\begin{array}{l}Q \\
Q \\
Q\end{array}$ & $\begin{array}{l}Q \\
Q \\
Q\end{array}$ & $\begin{array}{l}Q \\
Q \\
Q\end{array}$ & $\begin{array}{l}Q \\
Q \\
Q\end{array}$ & $\begin{array}{r}11.5 \\
12.0 \\
7.7\end{array}$ & $\begin{array}{l}Q \\
Q \\
Q\end{array}$ & $\begin{array}{l}25.8 \\
17.7 \\
18.6\end{array}$ \\
\hline
\end{tabular}

See footnotes at end of table. 
Table 5.15. U.S. Average Vehicle-Miles Traveled by Vehicle Type, 1994 (Continued)

(Thousand Miles per Vehicle)

\begin{tabular}{|c|c|c|c|c|c|c|c|c|c|c|}
\hline \multirow{4}{*}{$\begin{array}{l}1993 \text { Household and } \\
1994 \text { Vehicle } \\
\text { Characteristics }\end{array}$} & \multirow{3}{*}{$\begin{array}{l}\text { All } \\
\text { Vehicle } \\
\text { Types }\end{array}$} & \multicolumn{8}{|c|}{ Type of Vehicle } & \multirow[b]{4}{*}{$\begin{array}{l}\text { RSE } \\
\text { Row } \\
\text { Factor: }\end{array}$} \\
\hline & & \multicolumn{3}{|c|}{ Passenger Cars } & \multirow[b]{2}{*}{$\begin{array}{c}\text { Mini- } \\
\text { van }\end{array}$} & \multirow[b]{2}{*}{$\begin{array}{l}\text { Sport } \\
\text { Utility }\end{array}$} & \multirow[b]{2}{*}{$\begin{array}{c}\text { Large } \\
\text { Van }\end{array}$} & \multirow[b]{2}{*}{$\begin{array}{c}\text { Pickup } \\
\text { Truck }\end{array}$} & \multirow[b]{2}{*}{ Other } & \\
\hline & & All & Sedan & $\begin{array}{l}\text { Station } \\
\text { Wagon }\end{array}$ & & & & & & \\
\hline & 0.3 & 0.4 & 0.4 & 1.4 & 0.9 & 1.2 & 2.4 & 0.8 & 5.4 & \\
\hline $\begin{array}{l}\text { Household Composition } \\
\text { Two or More Adults ........................ } \\
\text { Age of Householder }\end{array}$ & 11.0 & 10.9 & 10.9 & 12.2 & 12.5 & 12.8 & 10.8 & 10.8 & $Q$ & 6.5 \\
\hline 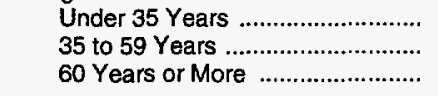 & $\begin{array}{r}12.7 \\
11.6 \\
9.3\end{array}$ & $\begin{array}{r}12.5 \\
11.5 \\
9.3\end{array}$ & $\begin{array}{r}12.5 \\
11.5 \\
9.2\end{array}$ & $\begin{array}{c}Q \\
11.4 \\
12.2\end{array}$ & $\begin{array}{c}Q \\
12.7 \\
11.3\end{array}$ & $\begin{array}{l}13.1 \\
12.6 \\
12.7\end{array}$ & $\begin{array}{r}Q \\
11.9 \\
9.1\end{array}$ & $\begin{array}{r}12.6 \\
11.5 \\
8.8\end{array}$ & $\begin{array}{l}Q \\
Q \\
Q\end{array}$ & $\begin{array}{l}17.8 \\
10.0 \\
10.7\end{array}$ \\
\hline $\begin{array}{l}\text { Race of Householder } \\
\text { White } \\
\text { Black } \\
\text { Other }\end{array}$ & $\begin{array}{l}11.5 \\
11.2 \\
10.9\end{array}$ & $\begin{array}{l}11.3 \\
11.1 \\
11.0\end{array}$ & $\begin{array}{l}11.3 \\
11.1 \\
10.8\end{array}$ & $\begin{array}{l}12.1 \\
Q\end{array}$ & $\begin{array}{c}13.6 \\
Q \\
Q\end{array}$ & $\begin{array}{l}12.5 \\
Q\end{array}$ & $\begin{array}{l}12.1 \\
Q \\
Q\end{array}$ & $\begin{array}{l}11.2 \\
10.7 \\
10.0\end{array}$ & $\begin{array}{l}7.1 \\
Q \\
Q\end{array}$ & $\begin{array}{r}4.3 \\
18.5 \\
24.3\end{array}$ \\
\hline 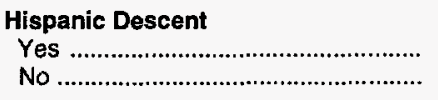 & $\begin{array}{l}11.4 \\
11.4\end{array}$ & $\begin{array}{l}11.4 \\
11.3\end{array}$ & $\begin{array}{l}11.2 \\
11.2\end{array}$ & $\begin{array}{l}Q \\
11.9\end{array}$ & $\begin{array}{l}11.5 \\
13.5\end{array}$ & $\begin{array}{l}12.4 \\
12.7\end{array}$ & $\stackrel{Q}{11.7}$ & $\begin{array}{l}11.1 \\
11.1\end{array}$ & $\frac{Q}{7.3}$ & $\begin{array}{r}19.2 \\
4.3\end{array}$ \\
\hline 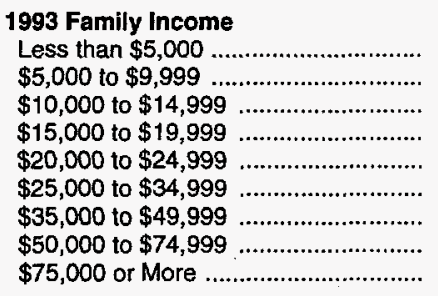 & $\begin{array}{r}11.5 \\
9.9 \\
10.2 \\
10.7 \\
11.0 \\
11.9 \\
11.6 \\
11.8 \\
12.3\end{array}$ & $\begin{array}{r}11.7 \\
9.8 \\
10.3 \\
10.6 \\
11.1 \\
11.9 \\
11.3 \\
11.7 \\
11.8\end{array}$ & $\begin{array}{r}11.6 \\
9.7 \\
10.3 \\
10.6 \\
11.0 \\
11.8 \\
11.3 \\
11.7 \\
11.8\end{array}$ & $\begin{array}{l}Q \\
Q \\
10.8 \\
11.1 \\
12.4 \\
12.2 \\
12.3 \\
13.0 \\
12.5\end{array}$ & $\begin{array}{c}Q \\
Q \\
Q \\
Q \\
12.7 \\
14.9 \\
13.8 \\
13.0 \\
13.5\end{array}$ & $\begin{array}{c}Q \\
Q \\
Q \\
10.8 \\
11.1 \\
12.8 \\
12.5 \\
13.9 \\
13.2\end{array}$ & $\begin{array}{c}Q \\
Q \\
Q \\
Q \\
Q \\
11.7 \\
12.6 \\
10.4 \\
Q\end{array}$ & $\begin{array}{c}Q \\
9.5 \\
9.6 \\
11.0 \\
10.6 \\
11.4 \\
11.3 \\
11.0 \\
13.3\end{array}$ & $\begin{array}{l}Q \\
Q \\
Q \\
Q \\
Q \\
Q \\
Q \\
Q \\
Q\end{array}$ & $\begin{array}{r}26.1 \\
29.9 \\
13.1 \\
16.8 \\
17.1 \\
9.5 \\
7.9 \\
8.9 \\
11.9\end{array}$ \\
\hline 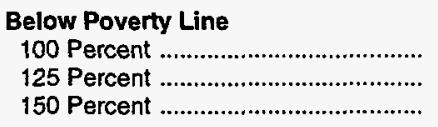 & $\begin{array}{l}10.7 \\
10.9 \\
11.1\end{array}$ & $\begin{array}{l}10.8 \\
11.0 \\
11.2\end{array}$ & $\begin{array}{l}10.8 \\
11.0 \\
11.2\end{array}$ & $\begin{array}{l}11.9 \\
11.4 \\
11.5\end{array}$ & $\begin{array}{c}\mathbf{Q} \\
12.5 \\
13.1\end{array}$ & $\begin{array}{c}Q \\
10.7 \\
10.5\end{array}$ & $\begin{array}{c}Q \\
Q \\
11.2\end{array}$ & $\begin{array}{r}9.5 \\
9.9 \\
10.3\end{array}$ & $\begin{array}{l}Q \\
Q \\
Q\end{array}$ & $\begin{array}{l}18.7 \\
14.5 \\
13.0\end{array}$ \\
\hline Eligible for Federal Assistance ${ }^{1} \ldots \ldots$ & 10.9 & 11.1 & 11.0 & 11.7 & 12.6 & 10.0 & 11.2 & 10.2 & $Q$ & 12.4 \\
\hline Number of Drivers ${ }^{2}$ (Fall 1993) & $\begin{array}{l}10.3 \\
11.6 \\
12.0 \\
12.8\end{array}$ & $\begin{array}{l}10.2 \\
11.4 \\
12.0 \\
12.9\end{array}$ & $\begin{array}{l}10.2 \\
11.3 \\
11.9 \\
12.9\end{array}$ & $\begin{array}{l}10.6 \\
12.5 \\
13.3 \\
Q\end{array}$ & $\begin{array}{c}10.8 \\
13.8 \\
14.9 \\
Q\end{array}$ & $\begin{array}{l}11.8 \\
12.9 \\
12.8 \\
11.3\end{array}$ & $\begin{array}{c}10.8 \\
10.7 \\
15.4 \\
Q\end{array}$ & $\begin{array}{l}10.3 \\
11.2 \\
10.7 \\
13.6\end{array}$ & $\begin{array}{l}Q \\
Q \\
Q \\
Q\end{array}$ & $\begin{array}{r}13.1 \\
5.1 \\
10.8 \\
16.4\end{array}$ \\
\hline 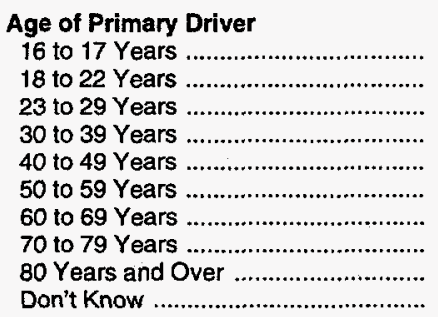 & $\begin{array}{r}9.6 \\
12.9 \\
13.3 \\
12.2 \\
12.4 \\
11.6 \\
9.3 \\
7.9 \\
6.1 \\
11.5\end{array}$ & $\begin{array}{r}10.1 \\
12.8 \\
13.0 \\
12.3 \\
12.2 \\
11.9 \\
9.1 \\
8.2 \\
5.4 \\
11.4\end{array}$ & $\begin{array}{r}10.1 \\
12.9 \\
13.0 \\
12.3 \\
12.1 \\
11.7 \\
9.1 \\
8.0 \\
5.4 \\
11.4\end{array}$ & $\begin{array}{l}Q \\
Q \\
Q \\
12.5 \\
12.8 \\
Q \\
Q \\
Q \\
Q \\
11.7\end{array}$ & $\begin{array}{c}Q \\
Q \\
Q \\
12.6 \\
15.6 \\
12.4 \\
Q \\
Q \\
Q \\
12.8\end{array}$ & $\begin{array}{c}Q \\
Q \\
15.9 \\
12.4 \\
12.7 \\
11.7 \\
Q \\
Q \\
Q \\
12.9\end{array}$ & $\begin{array}{c}Q \\
Q \\
Q \\
Q \\
13.6 \\
Q \\
Q \\
Q \\
Q \\
11.8\end{array}$ & $\begin{array}{c}Q \\
14.0 \\
12.8 \\
11.6 \\
11.6 \\
11.1 \\
9.1 \\
6.0 \\
Q \\
11.4\end{array}$ & $\begin{array}{l}Q \\
Q \\
Q \\
Q \\
Q \\
Q \\
Q \\
Q \\
Q \\
Q\end{array}$ & $\begin{array}{r}64.0 \\
17.0 \\
13.5 \\
8.4 \\
8.4 \\
12.1 \\
14.1 \\
17.1 \\
32.6 \\
11.5\end{array}$ \\
\hline
\end{tabular}

See footnotes at end of table. 
Table 5.15. U.S. Average Vehicle-Miles Traveled by Vehicle Type, 1994 (Continued)

(Thousand Miles per Vehicle)

\begin{tabular}{|c|c|c|c|c|c|c|c|c|c|c|}
\hline \multirow{3}{*}{$\begin{array}{l}1993 \text { Household and } \\
1994 \text { Vehicle } \\
\text { Characteristics }\end{array}$} & \multirow{3}{*}{$\begin{array}{c}\text { All } \\
\text { Vehicle } \\
\text { Types }\end{array}$} & \multicolumn{8}{|c|}{ Type of Vehicle } & \multirow[b]{4}{*}{$\begin{array}{l}\text { RSE } \\
\text { Row } \\
\text { Factor: }\end{array}$} \\
\hline & & \multicolumn{3}{|c|}{ Passenger Cars } & \multirow[b]{2}{*}{$\begin{array}{l}\text { Mini- } \\
\text { van }\end{array}$} & \multirow[b]{2}{*}{$\begin{array}{l}\text { Sport } \\
\text { Utility }\end{array}$} & \multirow[b]{2}{*}{$\begin{array}{l}\text { Large } \\
\text { Van }\end{array}$} & \multirow[b]{2}{*}{$\begin{array}{l}\text { Pickup } \\
\text { Truck }\end{array}$} & \multirow[b]{2}{*}{ Other } & \\
\hline & & All & Sedan & $\begin{array}{l}\text { Station } \\
\text { Wagon }\end{array}$ & & & & & & \\
\hline RSE Column Factor: & 0.3 & 0.4 & 0.4 & 1.4 & 0.9 & 1.2 & 2.4 & 0.8 & 5.4 & \\
\hline \multicolumn{11}{|l|}{ Sex of Primary Driver } \\
\hline Female & 11.7 & 11.2 & 11.2 & 12.2 & 14.1 & 14.0 & 15.4 & 11.0 & $Q$ & 6.4 \\
\hline Male …........................................... & 11.1 & 11.1 & 11.0 & 12.5 & 12.7 & 11.8 & 10.3 & 10.9 & $\mathrm{Q}$ & 6.3 \\
\hline 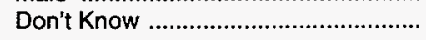 & 11.5 & 11.4 & 11.4 & 11.7 & 12.8 & 12.9 & 11.8 & 11.4 & $\vec{Q}$ & 11.5 \\
\hline \multicolumn{11}{|l|}{$\begin{array}{l}\text { Average Number of Vehicles per } \\
\text { Household During the Year }\end{array}$} \\
\hline 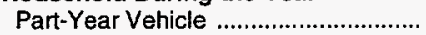 & 10.9 & 10.9 & 11.0 & $Q$ & $\mathrm{Q}$ & $\mathrm{Q}$ & $\mathrm{Q}$ & $\mathrm{Q}$ & $Q$ & 80.4 \\
\hline Only 1 & 10.7 & 10.5 & 10.4 & 11.3 & 12.5 & 12.6 & $\mathrm{Q}$ & 11.7 & $Q$ & 12.0 \\
\hline 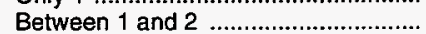 & 12.6 & 12.5 & 12.5 & 12.8 & 15.6 & 12.4 & $\mathrm{Q}$ & 12.7 & $\vec{Q}$ & 17.3 \\
\hline Only 2 & 11.6 & 11.3 & 11.3 & 11.9 & 13.7 & 12.9 & 13.3 & 11.0 & $\vec{Q}$ & 6.8 \\
\hline Between 2 and 3 ....................................... & 12.0 & 11.9 & 11.7 & 14.5 & 14.3 & 14.7 & $\mathrm{Q}$ & 11.1 & $\bar{Q}$ & 12.3 \\
\hline Only 3 & 10.9 & 10.8 & 10.7 & 13.1 & 13.9 & 12.2 & 10.5 & 10.4 & $\widehat{Q}$ & 11.9 \\
\hline Between 3 and 4 & 12.7 & 12.5 & 12.5 & $Q$ & $Q$ & 12.6 & $\mathrm{Q}$ & 13.7 & $\vec{Q}$ & 22.2 \\
\hline 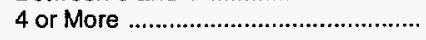 & 11.4 & 12.0 & 12.1 & 10.9 & 11.3 & 10.1 & $\mathrm{Q}$ & 10.3 & $\vec{Q}$ & 14.1 \\
\hline \multicolumn{11}{|l|}{ Vehicle Characteristics } \\
\hline \multicolumn{11}{|l|}{ Model Year } \\
\hline 1994 to 1995 & 14.3 & 13.9 & 13.8 & $\mathrm{Q}$ & 16.3 & 13.2 & Q & 14.8 & Q & 12.6 \\
\hline 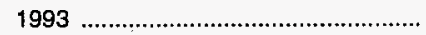 & 13.7 & 13.3 & 13.4 & 13.0 & 16.0 & 14.1 & Q & 13.9 & $Q$ & 11.4 \\
\hline 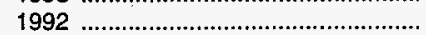 & 13.1 & 13.0 & 12.8 & $Q$ & 15.9 & 13.6 & $\bar{Q}$ & 12.5 & $\bar{Q}$ & 11.5 \\
\hline 1989 to 1991 & 12.4 & 11.8 & 11.8 & 12.6 & 13.2 & 14.6 & 16.0 & 13.3 & $Q$ & 7.2 \\
\hline 1986 to 1988 & 11.3 & 11.3 & 11.2 & 11.7 & 11.2 & 13.0 & 11.0 & 11.2 & $\bar{Q}$ & 9.1 \\
\hline 1983 to 1985 & 10.6 & 10.5 & 10.4 & 11.8 & 10.0 & 10.9 & 11.8 & 10.6 & $\bar{Q}$ & 12.2 \\
\hline 1980 to 1982 & 9.8 & 10.1 & 10.0 & 11.9 & $Q$ & $Q$ & $Q$ & 8.8 & $\bar{Q}$ & 22.4 \\
\hline 1977 to 1979 & 9.3 & 9.6 & 9.7 & $Q$ & $\mathrm{Q}$ & $\bar{Q}$ & $\mathrm{Q}$ & 8.9 & $\bar{Q}$ & 31.1 \\
\hline 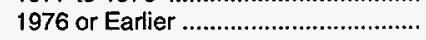 & 8.2 & 8.3 & 8.1 & $\bar{a}$ & $\bar{Q}$ & $\bar{Q}$ & $\vec{Q}$ & 8.4 & $\bar{Q}$ & 26.5 \\
\hline \multicolumn{11}{|l|}{ Fuel Efficiency (miles per gallon) } \\
\hline 10.9 or Less & 5.6 & 4.9 & 4.8 & a & $Q$ & 4.1 & $\mathrm{Q}$ & 5.6 & 7.1 & 21.4 \\
\hline 11 to 12.9 & 8.8 & 7.4 & 7.1 & $Q$ & $Q$ & 11.0 & Q & 9.8 & $\mathbf{Q}$ & 27.3 \\
\hline 13 to 15.9 & 9.7 & 7.2 & 7.3 & $\mathrm{Q}$ & $\bar{Q}$ & 12.5 & 12.5 & 11.1 & $\bar{Q}$ & 12.1 \\
\hline 16 to 18.9 & 10.5 & 8.6 & 8.4 & 11.8 & 11.2 & 13.5 & $Q$ & 12.5 & $\bar{Q}$ & 8.8 \\
\hline 19 to 21.9 & 12.0 & 11.2 & 11.1 & 12.6 & 15.2 & 15.8 & $Q$ & 12.1 & $Q$ & 7.6 \\
\hline 22 to 24.9 & 12.8 & 12.7 & 12.8 & 12.0 & 15.8 & $Q$ & $Q$ & 13.1 & $\mathrm{Q}$ & 7.0 \\
\hline 25 to 29.9 & 13.0 & 12.9 & 12.9 & 13.6 & $Q$ & $\vec{Q}$ & $\vec{Q}$ & 15.0 & $\hat{Q}$ & 8.2 \\
\hline 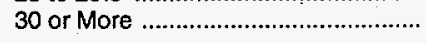 & 14.7 & 14.8 & 14.8 & $Q$ & $\bar{Q}$ & $\vec{Q}$ & $\mathrm{Q}$ & $Q$ & $\vec{Q}$ & 12.8 \\
\hline \multicolumn{11}{|l|}{ Engine Size (liters) } \\
\hline 2.49 or Less & 11.7 & 11.7 & 11.6 & 12.2 & 11.6 & 13.3 & $Q$ & 11.5 & $Q$ & 8.6 \\
\hline 2.50 to 3.49 & 12.1 & 11.6 & 11.6 & 12.3 & 13.9 & 12.5 & $\mathrm{Q}$ & 11.7 & $Q$ & 8.2 \\
\hline 3.50 to 4.49 & 11.7 & 11.1 & 11.2 & 10.7 & 13.3 & 13.4 & $\vec{Q}$ & 11.6 & $\mathbf{Q}$ & 11.1 \\
\hline 4.50 or Greater & 10.5 & 10.1 & 10.0 & 12.3 & $Q$ & 11.6 & 11.7 & 10.7 & 6.8 & 8.2 \\
\hline \multicolumn{11}{|l|}{ Number of Cylinders } \\
\hline 4 & 11.6 & 11.6 & 11.5 & 12.2 & 11.8 & 11.9 & $Q$ & 11.5 & $Q$ & 7.9 \\
\hline 6 & 12.1 & $\$ 1.6$ & 11.6 & 12.3 & 13.9 & 13.1 & 11.3 & 11.6 & $Q$ & 7.4 \\
\hline 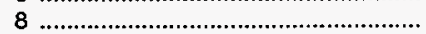 & 10.5 & 10.1 & 10.1 & 11.2 & $\mathrm{Q}$ & 11.9 & 11.8 & 10.6 & 6.7 & 8.4 \\
\hline 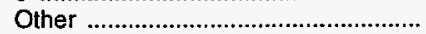 & 10.6 & 10.6 & 10.6 & $\mathbf{Q}$ & $Q$ & $Q$ & $\mathbf{Q}$ & $\mathbf{Q}$ & a & 50.1 \\
\hline
\end{tabular}

See footnotes at end of table. 
Table 5.15. U.S. Average Vehicle-Miles Traveled by Vehicle Type, 1994 (Continued)

(Thousand Miles per Vehicle)

\begin{tabular}{|c|c|c|c|c|c|c|c|c|c|c|}
\hline \multirow{3}{*}{$\begin{array}{l}1993 \text { Household and } \\
1994 \text { Vehicle } \\
\text { Characteristics }\end{array}$} & \multirow{3}{*}{$\begin{array}{l}\text { All } \\
\text { Vehicle } \\
\text { Types }\end{array}$} & \multicolumn{8}{|c|}{ Type of Vehicle } & \multirow[b]{4}{*}{$\begin{array}{l}\text { RSE } \\
\text { Row } \\
\text { Factor: }\end{array}$} \\
\hline & & \multicolumn{3}{|c|}{ Passenger Cars } & \multirow[b]{2}{*}{$\begin{array}{l}\text { Mini- } \\
\text { van }\end{array}$} & \multirow[b]{2}{*}{$\begin{array}{l}\text { Sport } \\
\text { Utility }\end{array}$} & \multirow[b]{2}{*}{$\begin{array}{l}\text { Large } \\
\text { Van }\end{array}$} & \multirow[b]{2}{*}{$\begin{array}{l}\text { Pickup } \\
\text { Truck }\end{array}$} & \multirow[b]{2}{*}{ Other } & \\
\hline & & All & Sedan & $\begin{array}{l}\text { Station } \\
\text { Wagon }\end{array}$ & & & & & & \\
\hline RSE Column Factor: & 0.3 & 0.4 & 0.4 & 1.4 & 0.9 & 1.2 & 2.4 & 0.8 & 5.4 & \\
\hline Type of Transmission & & & & & & & & & & \\
\hline Automatic & $\begin{array}{l}11.4 \\
11.6\end{array}$ & $\begin{array}{l}11.1 \\
12.0\end{array}$ & $\begin{array}{l}11.0 \\
12.0\end{array}$ & $\begin{array}{l}12.1 \\
12.0\end{array}$ & $\begin{array}{l}13.5 \\
12.5\end{array}$ & $\begin{array}{l}13.2 \\
11.3\end{array}$ & $\begin{array}{c}11.8 \\
Q\end{array}$ & $\begin{array}{l}11.1 \\
11.1\end{array}$ & $\mathrm{Q}^{7.4}$ & $\begin{array}{l}4.8 \\
9.2\end{array}$ \\
\hline 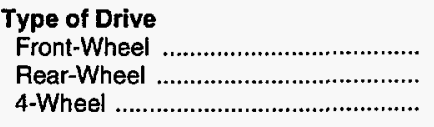 & $\begin{array}{l}11.9 \\
10.8 \\
11.9\end{array}$ & $\begin{array}{l}11.8 \\
10.4 \\
11.6\end{array}$ & $\begin{array}{l}11.7 \\
10.4 \\
11.4\end{array}$ & $\begin{array}{l}12.5 \\
11.5 \\
11.9\end{array}$ & $\begin{array}{c}13.7 \\
12.4 \\
Q\end{array}$ & $\begin{array}{l}Q \\
13.4 \\
12.4\end{array}$ & $\begin{array}{c}Q \\
11.8 \\
Q\end{array}$ & $\begin{array}{l}11.8 \\
11.0 \\
11.3\end{array}$ & $\begin{array}{l}Q \\
6.6 \\
Q\end{array}$ & $\begin{array}{r}5.4 \\
7.5 \\
12.4\end{array}$ \\
\hline 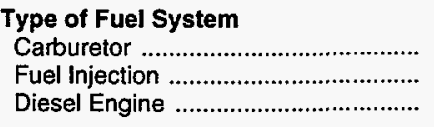 & $\begin{array}{l}10.5 \\
12.4 \\
11.5\end{array}$ & $\begin{array}{l}10.6 \\
11.9 \\
11.3\end{array}$ & $\begin{array}{c}10.4 \\
11.9 \\
11.3\end{array}$ & $\begin{array}{c}11.8 \\
12.6 \\
Q\end{array}$ & $\begin{array}{c}10.9 \\
14.0 \\
Q\end{array}$ & $\begin{array}{c}11.0 \\
13.8 \\
Q\end{array}$ & $\begin{array}{c}10.6 \\
15.0 \\
Q\end{array}$ & $\begin{array}{l}10.3 \\
12.7 \\
11.6\end{array}$ & $\begin{array}{l}Q \\
Q \\
Q\end{array}$ & $\begin{array}{r}7.6 \\
4.7 \\
32.8\end{array}$ \\
\hline 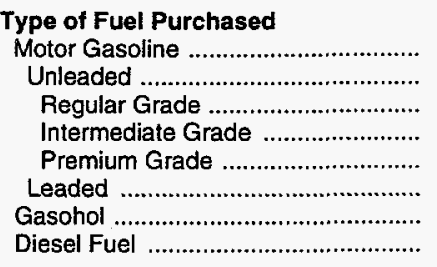 & $\begin{array}{l}11.4 \\
11.5 \\
11.5 \\
11.5 \\
11.2 \\
Q \\
11.7 \\
12.0\end{array}$ & $\begin{array}{l}11.3 \\
11.3 \\
11.5 \\
11.2 \\
10.8 \\
Q \\
12.3 \\
10.4\end{array}$ & $\begin{array}{l}11.2 \\
11.2 \\
11.4 \\
11.0 \\
10.8 \\
Q \\
12.3 \\
10.5\end{array}$ & $\begin{array}{l}12.1 \\
12.1 \\
12.0 \\
12.9 \\
11.8 \\
Q \\
Q \\
Q\end{array}$ & $\begin{array}{l}13.4 \\
13.4 \\
13.4 \\
13.0 \\
13.4 \\
Q \\
Q \\
Q\end{array}$ & $\begin{array}{l}12.7 \\
12.8 \\
12.8 \\
12.4 \\
13.0 \\
Q \\
Q \\
Q\end{array}$ & $\begin{array}{l}11.7 \\
11.9 \\
11.9 \\
Q \\
Q \\
Q \\
Q \\
Q\end{array}$ & $\begin{array}{c}11.0 \\
11.1 \\
10.8 \\
12.3 \\
11.4 \\
Q \\
Q \\
13.4\end{array}$ & $\begin{array}{l}7.0 \\
6.9 \\
Q \\
Q \\
Q \\
Q \\
Q \\
Q\end{array}$ & $\begin{array}{r}4.3 \\
4.2 \\
5.3 \\
10.1 \\
10.9 \\
100.0 \\
32.5 \\
40.6\end{array}$ \\
\hline 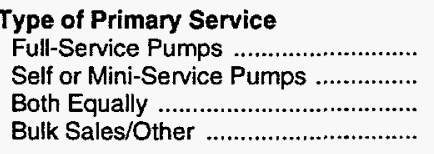 & $\begin{array}{c}9.9 \\
11.6 \\
10.3 \\
Q\end{array}$ & $\begin{array}{c}9.9 \\
11.5 \\
10.2 \\
Q\end{array}$ & $\begin{array}{r}9.9 \\
11.4 \\
10.1 \\
Q\end{array}$ & $\begin{array}{c}10.5 \\
12.3 \\
Q \\
Q\end{array}$ & $\begin{array}{c}10.4 \\
13.8 \\
Q \\
Q\end{array}$ & $\begin{array}{l}11.0 \\
12.8 \\
Q \\
Q\end{array}$ & $\begin{array}{c}Q \\
11.6 \\
Q \\
Q\end{array}$ & $\begin{array}{c}8.7 \\
11.3 \\
Q \\
Q\end{array}$ & $\begin{array}{l}Q \\
7.1 \\
Q \\
Q\end{array}$ & $\begin{array}{r}19.9 \\
4.3 \\
36.1 \\
Q\end{array}$ \\
\hline $\begin{array}{l}\text { Vehicle Use for Commuting } \\
\text { to and from Work } \\
\text { Yes }\end{array}$ & $\begin{array}{l}12.3 \\
10.0\end{array}$ & $\begin{array}{r}12.2 \\
9.9\end{array}$ & $\begin{array}{r}12.2 \\
9.8\end{array}$ & $\begin{array}{l}12.8 \\
10.9\end{array}$ & $\begin{array}{l}14.2 \\
12.2\end{array}$ & $\begin{array}{l}13.1 \\
11.6\end{array}$ & $\begin{array}{l}13.0 \\
10.2\end{array}$ & $\begin{array}{r}11.9 \\
9.8\end{array}$ & $\begin{array}{l}Q \\
7.1\end{array}$ & $\begin{array}{l}4.8 \\
7.4\end{array}$ \\
\hline
\end{tabular}

1 Below 150 percent of poverty line or 60 percent of median State income.

2 Approximately .5 percent of the vehicle stock was owned by households that had no drivers as of fall 1993.

NF $=$ No applicable RSE row factor.

$Q=$ Data withheld either because the Relative Standard Error (RSE) was greater than 50 percent or fewer than 10 households were sampled.

Notes: "Households with Children" category includesmembers under age 18 unless the member is the householder or spouse.* To obtain the RSE percentage for any table cell, multiply the corresponding column and row factors. - Because of rounding, data may not sum to totals. - Data in this table are for households with vehicles for personal use. - Percentages are calculated on unrounded numbers. - See Glossary for definition of terms used in this report.

Source: Energy Information Administration, Office of Energy Markets and End Use, Form ElA-457 A of the 1993 Residential Energy Consumption Survey and Forms EIA-876 A, B, C, and D for the 1994 Residential Transportation Energy Consumption Survey. 
Table 5.16. U.S. Number of Vehicles by Vehicle Type, 1994 (Million Vehicles)

\begin{tabular}{|c|c|c|c|c|c|c|c|c|c|c|}
\hline \multirow[b]{3}{*}{$\begin{array}{c}1993 \text { Household and } \\
1994 \text { Vehicle } \\
\text { Characteristics }\end{array}$} & \multirow{3}{*}{$\begin{array}{c}\text { All } \\
\text { Vehicle } \\
\text { Types }\end{array}$} & \multicolumn{8}{|c|}{ Type of Vehicle } & \multirow{4}{*}{$\begin{array}{l}\text { RSE } \\
\text { Row } \\
\text { Factor: }\end{array}$} \\
\hline & & \multicolumn{3}{|c|}{ Passenger Cars } & \multirow[b]{2}{*}{$\begin{array}{c}\text { Mini- } \\
\text { van }\end{array}$} & \multirow[b]{2}{*}{$\begin{array}{l}\text { Sport } \\
\text { Utility }\end{array}$} & \multirow[b]{2}{*}{$\begin{array}{c}\text { Large } \\
\text { Van }\end{array}$} & \multirow[b]{2}{*}{$\begin{array}{l}\text { Pickup } \\
\text { Truck }\end{array}$} & \multirow[b]{2}{*}{ Other } & \\
\hline & & All & Sedan & $\begin{array}{l}\text { Station } \\
\text { Wagon }\end{array}$ & & & & & & \\
\hline RSE Column Factor: & 0.4 & 0.4 & 0.5 & 1.4 & 1.3 & 1.2 & 2.0 & 0.8 & \multirow{2}{*}{4.0} & \\
\hline Household Characteristics & & & & & & & & & & \\
\hline Total & 156.8 & 106.4 & 100.3 & 6.2 & 8.1 & 9.5 & 3.4 & 28.8 & 0.6 & 6.7 \\
\hline \multicolumn{11}{|l|}{ Census Region and Division } \\
\hline 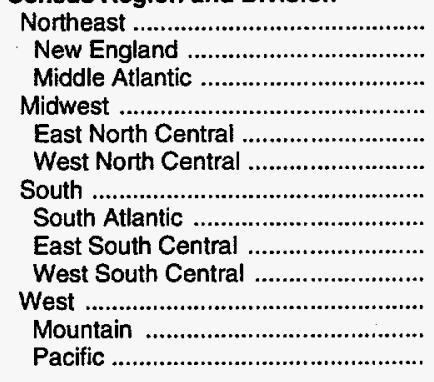 & $\begin{array}{r}26.6 \\
7.6 \\
19.0 \\
41.1 \\
29.0 \\
12.1 \\
56.0 \\
28.4 \\
11.1 \\
16.4 \\
33.1 \\
9.8 \\
23.3\end{array}$ & $\begin{array}{r}19.7 \\
5.3 \\
14.4 \\
28.7 \\
20.2 \\
8.5 \\
37.3 \\
19.7 \\
7.2 \\
10.4 \\
20.8 \\
5.5 \\
15.3\end{array}$ & $\begin{array}{r}17.9 \\
4.6 \\
13.3 \\
27.1 \\
19.2 \\
7.9 \\
35.7 \\
18.7 \\
7.0 \\
10.1 \\
19.6 \\
5.3 \\
14.3\end{array}$ & $\begin{array}{l}1.9 \\
0.7 \\
1.2 \\
1.6 \\
1.0 \\
0.6 \\
1.5 \\
1.0 \\
Q \\
Q \\
1.2 \\
Q \\
1.0\end{array}$ & $\begin{array}{l}1.3 \\
Q \\
0.9 \\
2.5 \\
1.8 \\
0.6 \\
2.7 \\
1.5 \\
0.4 \\
0.7 \\
1.6 \\
Q \\
1.1\end{array}$ & $\begin{array}{l}1.7 \\
0.6 \\
1.1 \\
1.5 \\
1.1 \\
Q \\
3.4 \\
1.9 \\
0.5 \\
1.0 \\
2.9 \\
1.0 \\
1.8\end{array}$ & $\begin{array}{l}Q \\
Q \\
Q \\
1.3 \\
1.1 \\
Q \\
1.1 \\
0.6 \\
Q \\
Q \\
0.7 \\
Q \\
Q\end{array}$ & $\begin{array}{r}3.5 \\
1.2 \\
2.3 \\
7.1 \\
4.7 \\
2.4 \\
11.3 \\
4.7 \\
2.7 \\
3.9 \\
6.8 \\
2.3 \\
4.6\end{array}$ & $\begin{array}{l}Q \\
Q \\
Q \\
Q \\
Q \\
Q \\
Q \\
Q \\
Q \\
Q \\
Q \\
Q \\
Q\end{array}$ & $\begin{array}{l}12.4 \\
27.1 \\
12.9 \\
11.1 \\
13.3 \\
18.0 \\
12.3 \\
15.3 \\
19.0 \\
28.4 \\
14.7 \\
19.1 \\
19.5\end{array}$ \\
\hline $\begin{array}{l}\text { Largest Populated States } \\
\text { Califomia } \ldots \ldots \ldots \ldots \ldots \ldots \ldots \ldots \ldots \ldots \ldots \ldots \ldots \\
\text { Florida } \\
\text { New York } \\
\text { Texas }\end{array}$ & $\begin{array}{r}17.0 \\
8.8 \\
7.8 \\
11.0\end{array}$ & $\begin{array}{r}11.1 \\
6.3 \\
6.0 \\
7.1\end{array}$ & $\begin{array}{r}10.5 \\
5.9 \\
5.5 \\
6.9\end{array}$ & $\begin{array}{l}0.6 \\
Q \\
0.5 \\
Q\end{array}$ & $\begin{array}{l}0.9 \\
0.5 \\
Q \\
Q\end{array}$ & $\begin{array}{l}1.3 \\
0.7 \\
Q \\
0.8\end{array}$ & $\begin{array}{l}Q \\
Q \\
Q \\
Q\end{array}$ & $\begin{array}{l}3.2 \\
1.1 \\
0.8 \\
2.4\end{array}$ & $\begin{array}{l}Q \\
Q \\
Q \\
Q\end{array}$ & $\begin{array}{l}24.2 \\
18.4 \\
16.8 \\
35.5\end{array}$ \\
\hline 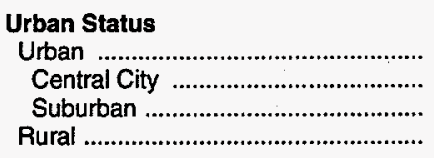 & $\begin{array}{r}119.4 \\
40.1 \\
79.3 \\
37.4\end{array}$ & $\begin{array}{l}84.1 \\
29.2 \\
55.0 \\
22.3\end{array}$ & $\begin{array}{l}79.1 \\
27.5 \\
51.6 \\
21.2\end{array}$ & $\begin{array}{l}5.0 \\
1.6 \\
3.4 \\
1.2\end{array}$ & $\begin{array}{l}6.5 \\
2.2 \\
4.4 \\
1.5\end{array}$ & $\begin{array}{l}7.5 \\
2.3 \\
5.3 \\
2.0\end{array}$ & $\begin{array}{l}2.7 \\
1.0 \\
1.8 \\
0.6\end{array}$ & $\begin{array}{r}18.1 \\
5.3 \\
12.7 \\
10.7\end{array}$ & $\begin{array}{l}Q \\
Q \\
Q \\
Q\end{array}$ & $\begin{array}{r}7.9 \\
15.9 \\
10.0 \\
12.9\end{array}$ \\
\hline 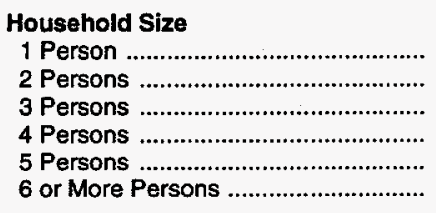 & $\begin{array}{r}19.5 \\
55.4 \\
31.7 \\
29.5 \\
14.4 \\
6.3\end{array}$ & $\begin{array}{r}15.6 \\
39.2 \\
21.3 \\
18.0 \\
8.5 \\
3.9\end{array}$ & $\begin{array}{r}14.8 \\
37.7 \\
19.8 \\
16.7 \\
7.7 \\
3.5\end{array}$ & $\begin{array}{l}0.8 \\
1.5 \\
1.5 \\
1.3 \\
0.7 \\
Q\end{array}$ & $\begin{array}{l}Q \\
1.3 \\
1.4 \\
2.6 \\
1.9 \\
0.7\end{array}$ & $\begin{array}{l}0.6 \\
2.9 \\
2.2 \\
2.2 \\
1.3 \\
Q\end{array}$ & $\begin{array}{l}Q \\
0.9 \\
0.5 \\
1.0 \\
0.4 \\
Q\end{array}$ & $\begin{array}{r}2.7 \\
10.6 \\
6.3 \\
5.7 \\
2.2 \\
1.3\end{array}$ & $\begin{array}{l}Q \\
Q \\
Q \\
Q \\
Q \\
Q\end{array}$ & $\begin{array}{l}16.7 \\
11.9 \\
15.7 \\
15.1 \\
23.2 \\
39.1\end{array}$ \\
\hline $\begin{array}{l}\text { Household Composition } \\
\text { Households with Children } \\
\text { Age of Oldest Child }\end{array}$ & 64.6 & 39.9 & 36.8 & 3.1 & 6.1 & 4.8 & 1.7 & 12.0 & $\mathbf{Q}$ & 10.3 \\
\hline 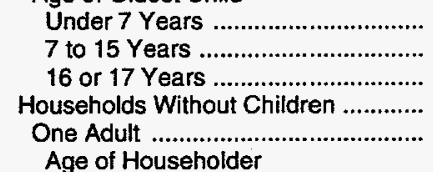 & $\begin{array}{l}17.1 \\
32.3 \\
15.2 \\
92.2 \\
19.5\end{array}$ & $\begin{array}{r}11.2 \\
18.9 \\
9.8 \\
66.6 \\
15.6\end{array}$ & $\begin{array}{r}10.4 \\
17.3 \\
9.1 \\
63.5 \\
14.8\end{array}$ & $\begin{array}{l}0.8 \\
1.7 \\
0.7 \\
3.1 \\
0.8\end{array}$ & $\begin{array}{l}1.3 \\
3.8 \\
1.1 \\
2.0 \\
Q\end{array}$ & $\begin{array}{l}1.5 \\
2.5 \\
0.9 \\
4.7 \\
0.6\end{array}$ & $\begin{array}{l}Q \\
1.0 \\
Q \\
1.7 \\
Q\end{array}$ & $\begin{array}{r}2.9 \\
6.0 \\
3.0 \\
16.8 \\
2.7\end{array}$ & $\begin{array}{l}Q \\
Q \\
Q \\
Q \\
Q\end{array}$ & $\begin{array}{r}21.4 \\
13.3 \\
24.3 \\
9.3 \\
16.7\end{array}$ \\
\hline 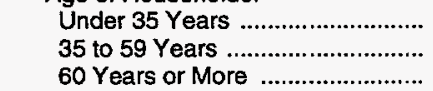 & $\begin{array}{l}4.1 \\
7.6 \\
7.9\end{array}$ & $\begin{array}{l}3.0 \\
5.6 \\
7.0\end{array}$ & $\begin{array}{l}2.9 \\
5.3 \\
6.6\end{array}$ & $\begin{array}{l}Q \\
Q \\
Q\end{array}$ & $\begin{array}{l}Q \\
Q \\
Q\end{array}$ & $\begin{array}{l}\mathbf{Q} \\
\mathbf{Q} \\
\mathbf{Q}\end{array}$ & $\begin{array}{l}Q \\
Q \\
Q\end{array}$ & $\begin{array}{l}0.7 \\
1.2 \\
0.8\end{array}$ & $\begin{array}{l}Q \\
Q \\
Q\end{array}$ & $\begin{array}{l}37.6 \\
28.8 \\
22.8\end{array}$ \\
\hline
\end{tabular}

See footnotes at end of table. 
Table 5.16. U.S. Number of Vehicles by Vehicle Type, 1994 (Continued) (Million Vehicles)

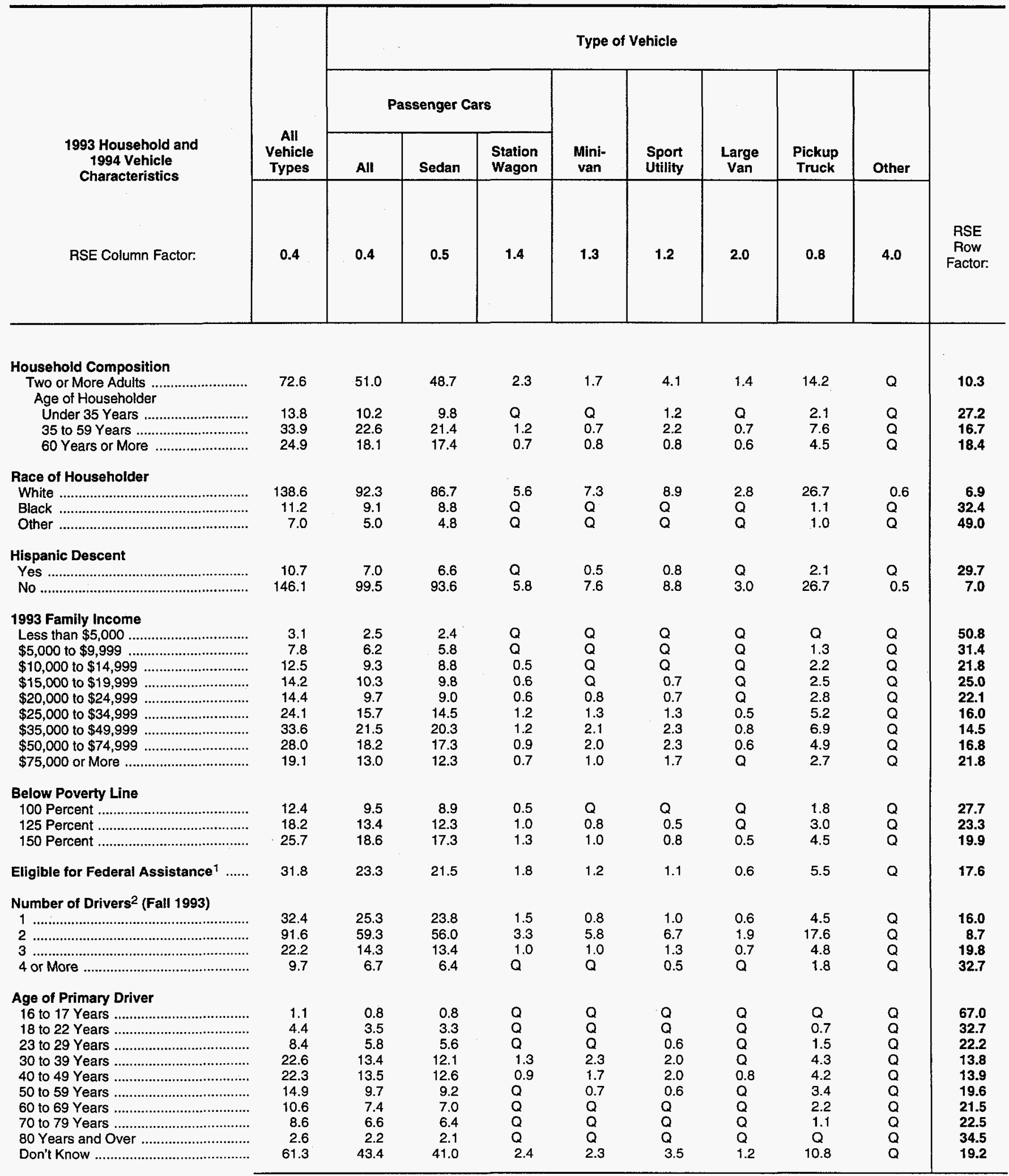

See footnotes at end of table. 
Table 5.16. U.S. Number of Vehicles by Vehicle Type, 1994 (Continued) (Million Vehicles)

\begin{tabular}{|c|c|c|c|c|c|c|c|c|c|c|}
\hline \multirow{3}{*}{$\begin{array}{l}1993 \text { Household and } \\
1994 \text { Vehicle } \\
\text { Characteristics }\end{array}$} & \multirow{3}{*}{$\begin{array}{c}\text { All } \\
\text { Vehicle } \\
\text { Types } \\
\end{array}$} & \multicolumn{8}{|c|}{ Type of Vehicle } & \multirow[b]{4}{*}{$\begin{array}{l}\text { RSE } \\
\text { Row } \\
\text { Factor }\end{array}$} \\
\hline & & \multicolumn{3}{|c|}{ Passenger Cars } & \multirow[b]{2}{*}{$\begin{array}{c}\text { Mini- } \\
\text { van }\end{array}$} & \multirow[b]{2}{*}{$\begin{array}{l}\text { Sport } \\
\text { Utility }\end{array}$} & \multirow[b]{2}{*}{$\begin{array}{c}\text { Large } \\
\text { Van }\end{array}$} & \multirow[b]{2}{*}{$\begin{array}{c}\text { Pickup } \\
\text { Truck }\end{array}$} & \multirow[b]{2}{*}{ Other } & \\
\hline & & All & Sedan & $\begin{array}{c}\text { Station } \\
\text { Wagon } \\
\end{array}$ & & & & & & \\
\hline RSE Column Factor: & 0.4 & 0.4 & 0.5 & 1.4 & 1.3 & 1.2 & 2.0 & 0.8 & 4.0 & \\
\hline \multicolumn{11}{|l|}{ Sex of Primary Driver } \\
\hline Female & 43.4 & 34.8 & 32.7 & 2.1 & 4.0 & 2.1 & 0.6 & 1.9 & $Q$ & 9.9 \\
\hline Male & 52.2 & 28.5 & 26.8 & 1.7 & 1.7 & 4.0 & 1.6 & 16.1 & $\mathbf{Q}$ & 9.8 \\
\hline 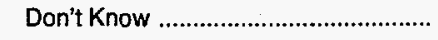 & 61.2 & 43.2 & 40.8 & 2.4 & 2.3 & 3.5 & 1.2 & 10.8 & $\mathbf{Q}$ & 19.3 \\
\hline \multicolumn{11}{|l|}{$\begin{array}{l}\text { Average Number of Vehicles per } \\
\text { Household During the Year }\end{array}$} \\
\hline 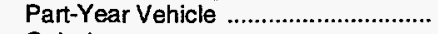 & 1.3 & 1.2 & 1.1 & Q & Q & $\mathbf{Q}$ & $Q$ & Q & $\mathbf{Q}$ & 88.4 \\
\hline Only 1 & 28.6 & 23.5 & 22.2 & 1.3 & 1.1 & 0.9 & $\mathbf{Q}$ & 2.7 & $\mathbf{Q}$ & 15.8 \\
\hline Between 1 and 2 & 8.4 & 5.9 & 5.5 & 0.4 & 0.5 & 0.6 & $Q$ & 1.3 & $\mathbf{Q}$ & 28.2 \\
\hline Only 2 & 56.9 & 37.0 & 34.7 & 2.3 & 3.5 & 4.0 & 1.2 & 11.1 & $\hat{Q}$ & 11.1 \\
\hline Between 2 and 3 & 13.5 & 8.6 & 8.2 & 0.4 & 0.9 & 1.2 & $Q$ & 2.6 & $\overrightarrow{\mathrm{Q}}$ & 20.4 \\
\hline Only 3 & 25.2 & 15.9 & 15.0 & 0.9 & 1.0 & 1.3 & 0.8 & 6.1 & $\mathbf{Q}$ & 17.8 \\
\hline Between 3 and $4 \ldots \ldots \ldots \ldots \ldots \ldots \ldots \ldots \ldots \ldots$ & 7.9 & 5.2 & 4.9 & $\mathbf{Q}$ & $\mathbf{Q}$ & 0.5 & $\mathbf{Q}$ & 1.7 & $\bar{Q}$ & 32.3 \\
\hline 4 or More & 14.8 & 9.3 & 8.6 & 0.6 & 0.7 & 1.0 & $\mathbf{Q}$ & 3.3 & $\mathbf{Q}$ & 25.2 \\
\hline \multicolumn{11}{|l|}{ Vehicle Characteristics } \\
\hline \multicolumn{11}{|l|}{ Model Year } \\
\hline 1994 to 1995 & 7.2 & 4.1 & 4.0 & $\mathbf{Q}$ & 0.8 & 0.8 & $\mathbf{Q}$ & 1.5 & $Q$ & 21.8 \\
\hline 1993 & 10.4 & 6.9 & 6.3 & 0.5 & 1.0 & 1.0 & Q & 1.4 & $\mathbf{Q}$ & 20.2 \\
\hline 1992 & 11.4 & 7.8 & 7.6 & $\mathbf{Q}$ & 0.8 & 1.0 & $\mathbf{Q}$ & 1.6 & $\bar{Q}$ & 20.0 \\
\hline 1989 to 1991 & 35.0 & 23.6 & 23.0 & 0.7 & 2.9 & 2.5 & 0.7 & 5.2 & $\mathbf{Q}$ & 12.7 \\
\hline 1986 to 1988 & 36.3 & 26.1 & 24.6 & 1.5 & 1.9 & 2.0 & $\mathbf{Q}$ & 5.6 & $\mathbf{Q}$ & 12.8 \\
\hline 1983 to 1985 & 25.6 & 18.2 & 16.3 & 1.9 & 0.5 & 1.1 & 0.8 & 4.7 & $\mathbf{Q}$ & 15.8 \\
\hline 1980 to 1982 & 10.9 & 8.1 & 7.4 & 0.7 & $\mathbf{Q}$ & $\mathbf{Q}$ & $\mathbf{Q}$ & 2.1 & $\mathbf{Q}$ & 23.5 \\
\hline 1977 to 1979 & 9.5 & 5.6 & 5.2 & $\mathbf{Q}$ & $\mathbf{Q}$ & $\mathbf{Q}$ & $\mathbf{Q}$ & 3.2 & $\mathbf{Q}$ & 24.0 \\
\hline 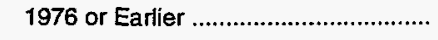 & 10.6 & 6.0 & 5.8 & $Q$ & $Q$ & 0.6 & $Q$ & 3.5 & $\mathbf{Q}$ & 29.3 \\
\hline \multicolumn{11}{|l|}{ Fuel Efficiency (miles per gallon) } \\
\hline 10.9 or Less & 7.0 & 1.9 & 1.9 & $\mathrm{Q}$ & $\mathbf{Q}$ & 0.7 & 0.6 & 3.3 & 0.6 & 21.1 \\
\hline 11 to 12.9 & 8.9 & 3.7 & 3.6 & $Q$ & $Q$ & 1.0 & Q & 3.5 & $\mathbf{Q}$ & 22.4 \\
\hline 13 to 15.9 & 18.9 & 7.7 & 7.2 & Q & $Q$ & 1.8 & 1.8 & 7.5 & $\mathbf{Q}$ & 15.2 \\
\hline 16 to 18.9 & 26.5 & 13.9 & 13.1 & 0.8 & 2.8 & 4.2 & $Q$ & 5.2 & $\mathrm{Q}$ & 14.2 \\
\hline 19 to 21.9 & 35.2 & 24.2 & 23.1 & 1.1 & 4.4 & 1.6 & $\mathbf{Q}$ & 4.9 & $\mathbf{Q}$ & 13.5 \\
\hline 22 to 24.9 & 28.2 & 24.4 & 22.7 & 1.7 & 0.6 & $\mathbf{Q}$ & $\mathbf{Q}$ & 3.1 & $\mathbf{Q}$ & 15.9 \\
\hline 25 to 29.9 & 24.9 & 23.6 & 22.2 & 1.4 & $Q$ & $Q$ & $\mathbf{Q}$ & 1.2 & $Q$ & 14.8 \\
\hline 30 or More & 7.2 & 7.0 & 6.6 & a & $\mathbf{Q}$ & $\mathbf{Q}$ & $Q$ & $Q$ & $\mathbf{Q}$ & 29.5 \\
\hline \multicolumn{11}{|l|}{ Engine Size (liters) } \\
\hline 2.49 or Less & 59.8 & 51.3 & 47.7 & 3.6 & 1.1 & 0.6 & Q & 6.7 & Q & 12.7 \\
\hline 2.50 to 3.49 & 30.1 & 19.4 & 18.4 & 1.0 & 4.8 & 2.3 & Q & 3.5 & Q & 12.6 \\
\hline 3.50 to 4.49 & 24.0 & 14.5 & 13.8 & 0.7 & 2.1 & 3.9 & $Q$ & 3.1 & $\mathbf{Q}$ & 15.9 \\
\hline 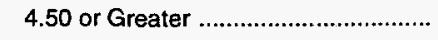 & 42.8 & 21.2 & 20.2 & 0.9 & $Q$ & 2.6 & 2.9 & 15.4 & 0.5 & 12.2 \\
\hline \multicolumn{11}{|l|}{ Number of Cylinders } \\
\hline 4 & 61.5 & 51.3 & 47.7 & 3.6 & 1.9 & 1.0 & $\mathbf{Q}$ & 7.4 & $\mathbf{Q}$ & 11.3 \\
\hline 6 & 52.3 & 31.3 & 29.7 & 1.6 & 6.2 & 6.1 & 0.8 & 8.0 & $Q$ & 10.8 \\
\hline 8 & 41.6 & 22.6 & 21.6 & 1.0 & $Q$ & 2.5 & 2.6 & 13.4 & 0.5 & 12.5 \\
\hline Other & 1.3 & 1.3 & 1.3 & $\mathbf{Q}$ & $Q$ & $\mathbf{Q}$ & $\mathbf{Q}$ & $Q$ & $\mathbf{Q}$ & 56.6 \\
\hline
\end{tabular}

See footnotes at end of table. 
Table 5.16. U.S. Number of Vehicles by Vehicle Type, 1994 (Continued)

(Million Vehicles)

\begin{tabular}{|c|c|c|c|c|c|c|c|c|c|c|}
\hline \multirow[b]{3}{*}{$\begin{array}{c}1993 \text { Household and } \\
1994 \text { Vehicle } \\
\text { Characteristics }\end{array}$} & \multirow{3}{*}{$\begin{array}{l}\text { All } \\
\text { Vehicle } \\
\text { Types }\end{array}$} & \multicolumn{8}{|c|}{ Type of Vehicle } & \multirow{4}{*}{$\begin{array}{l}\text { RSE } \\
\text { Row } \\
\text { Factor: }\end{array}$} \\
\hline & & \multicolumn{3}{|c|}{ Passenger Cars } & \multirow[b]{2}{*}{$\begin{array}{l}\text { Mini- } \\
\text { van }\end{array}$} & \multirow[b]{2}{*}{$\begin{array}{l}\text { Sport } \\
\text { Utility }\end{array}$} & \multirow[b]{2}{*}{$\begin{array}{c}\text { Large } \\
\text { Van }\end{array}$} & \multirow[b]{2}{*}{$\begin{array}{c}\text { Pickup } \\
\text { Truck }\end{array}$} & \multirow[b]{2}{*}{ Other } & \\
\hline & & All & Sedan & $\begin{array}{l}\text { Station } \\
\text { Wagon }\end{array}$ & & & & & & \\
\hline RSE Column Factor: & 0.4 & 0.4 & 0.5 & 1.4 & 1.3 & 1.2 & 2.0 & 0.8 & 4.0 & \\
\hline 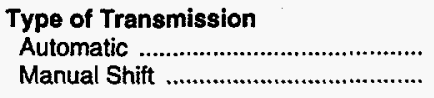 & $\begin{array}{r}117.5 \\
39.3\end{array}$ & $\begin{array}{l}84.8 \\
21.7\end{array}$ & $\begin{array}{l}80.3 \\
19.9\end{array}$ & $\begin{array}{l}4.5 \\
1.7\end{array}$ & $\begin{array}{l}7.5 \\
0.6\end{array}$ & $\begin{array}{l}7.0 \\
2.5\end{array}$ & $\begin{array}{l}3.2 \\
Q\end{array}$ & $\begin{array}{l}14.6 \\
14.2\end{array}$ & $\begin{array}{l}0.5 \\
Q\end{array}$ & $\begin{array}{r}7.5 \\
15.0\end{array}$ \\
\hline 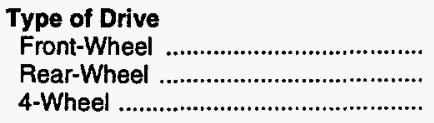 & $\begin{array}{l}74.9 \\
64.3 \\
17.6\end{array}$ & $\begin{array}{r}66.9 \\
37.8 \\
1.7\end{array}$ & $\begin{array}{r}63.5 \\
35.8 \\
1.0\end{array}$ & $\begin{array}{l}3.5 \\
2.0 \\
0.7\end{array}$ & $\begin{array}{l}5.1 \\
2.5 \\
Q\end{array}$ & $\begin{array}{l}Q \\
2.0 \\
7.1\end{array}$ & $\begin{array}{l}Q \\
3.1 \\
Q\end{array}$ & $\begin{array}{r}2.2 \\
18.4 \\
8.3\end{array}$ & $\begin{array}{l}0 \\
0.5 \\
Q\end{array}$ & $\begin{array}{r}9.6 \\
10.4 \\
21.2\end{array}$ \\
\hline 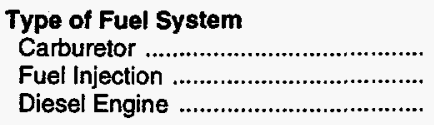 & $\begin{array}{r}76.4 \\
78.2 \\
2.2\end{array}$ & $\begin{array}{r}49.6 \\
55.9 \\
0.9\end{array}$ & $\begin{array}{r}45.5 \\
53.8 \\
0.9\end{array}$ & $\begin{array}{l}4.0 \\
2.1 \\
Q\end{array}$ & $\begin{array}{l}1.6 \\
6.5 \\
Q\end{array}$ & $\begin{array}{l}3.6 \\
5.9 \\
Q\end{array}$ & $\begin{array}{l}2.6 \\
0.8 \\
Q\end{array}$ & $\begin{array}{r}18.7 \\
8.8 \\
1.2\end{array}$ & $\begin{array}{l}Q \\
Q \\
a\end{array}$ & $\begin{array}{r}10.0 \\
9.2 \\
48.0\end{array}$ \\
\hline 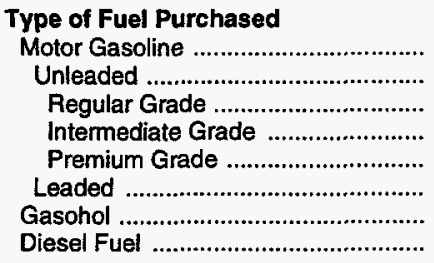 & $\begin{array}{r}153.4 \\
151.5 \\
104.2 \\
20.6 \\
26.7 \\
1.9 \\
1.4 \\
1.8\end{array}$ & $\begin{array}{c}104.8 \\
103.7 \\
69.8 \\
14.4 \\
19.5 \\
Q \\
0.9 \\
0.7\end{array}$ & $\begin{array}{l}98.6 \\
97.6 \\
65.5 \\
13.5 \\
18.6 \\
Q \\
0.9 \\
0.7\end{array}$ & $\begin{array}{l}6.2 \\
6.1 \\
4.3 \\
0.9 \\
0.9 \\
Q \\
Q \\
Q\end{array}$ & $\begin{array}{l}8.0 \\
8.0 \\
5.8 \\
1.1 \\
1.2 \\
Q \\
Q \\
Q\end{array}$ & $\begin{array}{l}9.4 \\
9.3 \\
5.9 \\
1.7 \\
1.7 \\
Q \\
Q \\
Q\end{array}$ & $\begin{array}{l}3.4 \\
3.3 \\
2.4 \\
Q \\
Q \\
Q \\
Q \\
Q\end{array}$ & $\begin{array}{c}27.3 \\
26.7 \\
19.9 \\
3.0 \\
3.8 \\
Q \\
Q \\
1.0\end{array}$ & $\begin{array}{l}0.5 \\
0.5 \\
Q \\
Q \\
Q \\
Q \\
Q \\
Q\end{array}$ & $\begin{array}{r}6.8 \\
6.8 \\
7.5 \\
16.4 \\
16.2 \\
77.9 \\
61.7 \\
54.5\end{array}$ \\
\hline 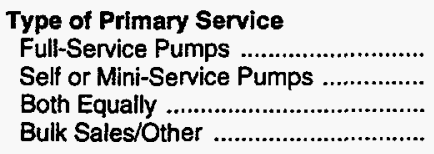 & $\begin{array}{r}13.8 \\
139.8 \\
2.9 \\
Q\end{array}$ & $\begin{array}{c}10.8 \\
93.6 \\
2.0 \\
Q\end{array}$ & $\begin{array}{c}10.0 \\
88.3 \\
1.9 \\
Q\end{array}$ & $\begin{array}{l}0.8 \\
5.3 \\
Q \\
Q\end{array}$ & $\begin{array}{l}0.7 \\
7.3 \\
Q \\
Q\end{array}$ & $\begin{array}{l}0.7 \\
8.6 \\
Q \\
Q\end{array}$ & $\begin{array}{l}Q \\
3.2 \\
Q \\
Q\end{array}$ & $\begin{array}{c}1.5 \\
26.5 \\
Q \\
Q\end{array}$ & $\begin{array}{l}Q \\
0.6 \\
Q \\
Q\end{array}$ & $\begin{array}{c}30.1 \\
7.3 \\
55.5 \\
Q\end{array}$ \\
\hline $\begin{array}{l}\text { Vehicle Use for Commuting } \\
\text { to and from Work } \\
\text { Yes }\end{array}$ & $\begin{array}{l}95.3 \\
61.5\end{array}$ & $\begin{array}{l}63.8 \\
42.6\end{array}$ & $\begin{array}{l}59.9 \\
40.3\end{array}$ & $\begin{array}{l}3.9 \\
2.3\end{array}$ & $\begin{array}{l}4.8 \\
3.2\end{array}$ & $\begin{array}{l}6.7 \\
2.9\end{array}$ & $\begin{array}{l}1.8 \\
1.6\end{array}$ & $\begin{array}{l}18.1 \\
10.6\end{array}$ & $\begin{array}{l}Q \\
0.6\end{array}$ & $\begin{array}{r}8.5 \\
10.0\end{array}$ \\
\hline
\end{tabular}

1 Below 150 percent of poverty line or 60 percent of median State income.

2 Approximately .5 percent of the vehicle stock was owned by households that had no drivers as of fall 1993.

$\mathrm{NF}=$ No applicable RSE row factor.

$\mathrm{Q}=$ Data withheld either because the Relative Standard Error (RSE) was greater than 50 percent or fewer than 10 households were sampled.

Notes: "Households with Children" category includesmembers under age 18 unless the member is the householder or spouse." To obtain the RSE percentage for any table cell, multiply the corresponding column and row factors. - Because of rounding, data may not sum to totals. $\bullet$ Data in this table are for households with vehicles for personal use. - Percentages are calculated on unrounded numbers. - See Glossary for definition of terms used in this report.

Source: Energy Information Administration, Office of Energy Markets and End Use, Form ElA-457 A of the 1993 Residential Energy Consumption Survey and Forms EIA-876 A, B, C, and D for the 1994 Residential Transportation Energy Consumption Survey. 
Table 5.17. U.S. Number of Households by Vehicle Fuel Expenditures, 1994

(Million Households)

\begin{tabular}{|c|c|c|c|c|c|c|c|c|c|c|c|c|}
\hline \multirow[b]{2}{*}{$\begin{array}{c}1993 \text { Household and } \\
1994 \text { Vehicle } \\
\text { Characteristics }\end{array}$} & \multirow[b]{2}{*}{$\begin{array}{c}\text { All } \\
\text { Expenditure } \\
\text { Categories }\end{array}$} & \multicolumn{5}{|c|}{$\begin{array}{l}\text { Motor Fuel Expenditures } \\
\text { (dollars per household) }\end{array}$} & \multicolumn{5}{|c|}{ Percent of Income for Motor Fuel } & \multirow[b]{3}{*}{$\begin{array}{l}\text { RSE } \\
\text { Row } \\
\text { Factor: }\end{array}$} \\
\hline & & $\begin{array}{l}\$ 500 \\
\text { or } \\
\text { Less }\end{array}$ & $\begin{array}{c}\$ 501 \\
\text { to } \\
\$ 1,000\end{array}$ & $\begin{array}{c}\$ 1,001 \\
\text { to } \\
\$ 1,500\end{array}$ & $\begin{array}{l}\$ 1,501 \\
\text { to } \\
\$ 2,000\end{array}$ & $\begin{array}{c}\$ 2,001 \\
\text { or } \\
\text { More }\end{array}$ & $\begin{array}{c}\text { Less } \\
\text { than } \\
2\end{array}$ & $\begin{array}{c}2 \\
\text { to } \\
3.9\end{array}$ & $\begin{array}{c}4 \\
\text { to } \\
5.9\end{array}$ & $\begin{array}{c}6 \\
\text { to } \\
7.9\end{array}$ & $\begin{array}{c}8 \\
\text { or } \\
\text { More }\end{array}$ & \\
\hline RSE Column Factor: & 0.5 & 1.1 & 0.9 & 1.1 & 1.2 & 1.1 & 1.0 & 0.9 & 1.1 & 1.6 & 1.2 & \\
\hline \multicolumn{13}{|l|}{ Household Characteristics } \\
\hline Total & 84.9 & 15.2 & 24.8 & 18.7 & 13.0 & 13.2 & 23.2 & 26.5 & 14.6 & 8.1 & 12.5 & 5.6 \\
\hline \multicolumn{13}{|l|}{ Census Region and Division } \\
\hline 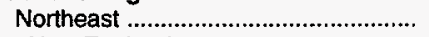 & 14.7 & 2.9 & 4.7 & 3.0 & 2.1 & 2.0 & 5.1 & 4.8 & 2.2 & 1.3 & 1.4 & 13.9 \\
\hline 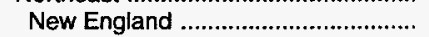 & 4.1 & 0.7 & 1.4 & 0.9 & 0.7 & $Q$ & 1.2 & 1.4 & 0.9 & $Q$ & $Q$ & 24.4 \\
\hline 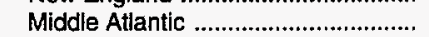 & 10.7 & 2.2 & 3.3 & 2.1 & 1.5 & 1.5 & 3.9 & 3.3 & 1.3 & 1.0 & 1.1 & 15.4 \\
\hline 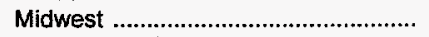 & 21.6 & 3.5 & 6.5 & 5.2 & 3.1 & 3.3 & 5.1 & 7.3 & 4.3 & 1.8 & 3.0 & 10.0 \\
\hline East North Central ............................... & 15.1 & 2.5 & 4.6 & 3.6 & 2.1 & 2.3 & 3.8 & 5.4 & 2.8 & 1.2 & 1.9 & 13.5 \\
\hline 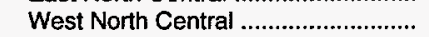 & 6.5 & 1.0 & 1.9 & 1.7 & 1.0 & 0.9 & 1.3 & 1.9 & 1.5 & 0.6 & 1.2 & 12.9 \\
\hline South & 30.2 & 5.8 & 8.0 & 6.8 & 4.7 & 4.9 & 7.2 & 9.4 & 5.7 & 3.1 & 4.9 & 9.7 \\
\hline 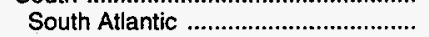 & 15.4 & 2.6 & 4.4 & 3.7 & 2.2 & 2.5 & 3.8 & 4.7 & 3.1 & 1.2 & 2.5 & 12.9 \\
\hline 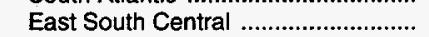 & 5.4 & 0.7 & 1.6 & 1.0 & 1.1 & 1.0 & 1.0 & 1.6 & 1.2 & 0.7 & 1.0 & 18.1 \\
\hline 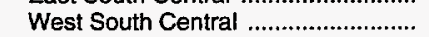 & 9.4 & 2.5 & 2.0 & 2.0 & 1.4 & 1.5 & 2.4 & 3.0 & 1.5 & 1.1 & 1.4 & 19.0 \\
\hline West & 18.4 & 2.9 & 5.6 & 3.7 & 3.1 & 3.0 & 5.7 & 5.0 & 2.5 & 1.9 & 3.2 & 12.5 \\
\hline 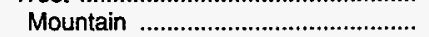 & 5.4 & 0.7 & 1.4 & 1.1 & 1.4 & 0.8 & 1.0 & 1.4 & 0.8 & 0.7 & 1.4 & 20.8 \\
\hline 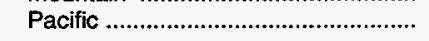 & 13.0 & 2.2 & 4.2 & 2.6 & 1.8 & 2.2 & 4.7 & 3.6 & 1.7 & 1.1 & 1.8 & 15.6 \\
\hline \multicolumn{13}{|l|}{ Largest Populated States } \\
\hline 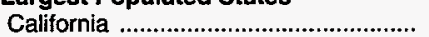 & 9.5 & 1.4 & 3.3 & 1.8 & 1.3 & 1.7 & 3.5 & 2.8 & 1.1 & 0.8 & 1.3 & 17.3 \\
\hline Florida & 4.9 & 0.9 & 1.4 & 1.3 & 0.7 & 0.7 & 1.3 & 1.4 & 1.2 & $Q$ & $Q$ & 16.9 \\
\hline 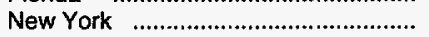 & 4.7 & 1.0 & 1.7 & 1.1 & 0.6 & $Q$ & 1.9 & 1.5 & 0.6 & $\vec{Q}$ & $\vec{Q}$ & 18.5 \\
\hline Texas & 6.1 & 1.6 & 1.3 & 1.3 & 1.0 & 0.9 & 1.7 & 1.9 & 0.9 & $Q$ & 1.0 & 21.5 \\
\hline \multicolumn{13}{|l|}{ Urban Status } \\
\hline 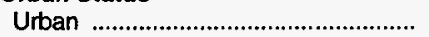 & 65.6 & 11.9 & 19.7 & 14.8 & 9.9 & 9.3 & 20.2 & 21.5 & 10.5 & 5.3 & 8.1 & 6.8 \\
\hline 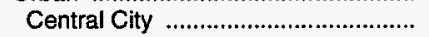 & 24.1 & 5.6 & 8.1 & 5.1 & 3.1 & 2.2 & 7.7 & 7.9 & 3.4 & 2.1 & 3.1 & 13.8 \\
\hline 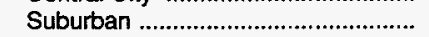 & 41.5 & 6.3 & 11.7 & 9.7 & 6.8 & 7.1 & 12.6 & 13.5 & 7.2 & 3.2 & 5.0 & 8.7 \\
\hline Rural & 19.3 & 3.2 & 5.1 & 3.9 & 3.1 & 3.9 & 3.0 & 5.0 & 4.1 & 2.8 & 4.4 & 11.0 \\
\hline \multicolumn{13}{|l|}{ Household Size } \\
\hline 1 Person & 17.0 & 7.7 & 6.5 & 1.9 & 0.6 & $Q$ & 6.3 & 4.8 & 2.6 & 1.3 & 2.0 & 13.0 \\
\hline 2 Persons & 30.1 & 5.0 & 9.4 & 7.4 & 4.7 & 3.6 & 8.9 & 9.4 & 4.9 & 3.0 & 3.9 & 9.0 \\
\hline 3 Persons & 15.0 & 1.2 & 3.7 & 4.1 & 2.7 & 3.4 & 3.2 & 4.9 & 2.7 & 1.5 & 2.7 & 14.1 \\
\hline 4 Persons & 13.3 & 0.8 & 3.1 & 3.4 & 2.7 & 3.3 & 2.9 & 4.7 & 2.4 & 1.2 & 2.1 & 15.1 \\
\hline 5 Persons & 6.7 & $Q$ & 1.6 & 1.3 & 1.6 & 1.7 & 1.3 & 2.1 & 1.4 & 0.8 & 1.1 & 21.3 \\
\hline 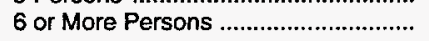 & 2.7 & $\widehat{Q}$ & $Q$ & $Q$ & 0.6 & 0.8 & $Q$ & 0.7 & 0.6 & $Q$ & 0.7 & 32.5 \\
\hline \multicolumn{13}{|l|}{ Household Composition } \\
\hline $\begin{array}{l}\text { Households with Children } \\
\text { Age of Oldest Child }\end{array}$ & 32.1 & 2.8 & 8.2 & 8.2 & 6.1 & 6.8 & 6.8 & 10.6 & 6.0 & 3.2 & 5.5 & 9.8 \\
\hline Under 7 Years .............................. & 9.2 & 1.0 & 2.6 & 2.7 & 1.8 & 1.3 & 2.4 & 3.3 & 1.5 & 0.8 & 1.3 & 21.4 \\
\hline 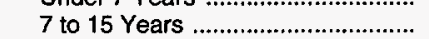 & 16.5 & 1.4 & 4.3 & 4.1 & 3.1 & 3.5 & 3.4 & 5.4 & 3.2 & 1.6 & 2.9 & 13.1 \\
\hline 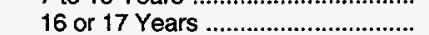 & 6.4 & $Q$ & 1.3 & 1.4 & 1.2 & 2.1 & 1.0 & 1.9 & 1.3 & 0.9 & 1.3 & 20.8 \\
\hline Households Without Children ............ & 52.7 & 12.4 & 16.6 & 10.5 & 6.9 & 6.4 & 16.4 & 15.9 & 8.6 & 4.9 & 7.0 & 7.3 \\
\hline $\begin{array}{l}\text { One Adult ............................................... } \\
\text { Age of Householder }\end{array}$ & 17.0 & 7.7 & 6.5 & 1.9 & 0.6 & $Q$ & 6.3 & 4.8 & 2.6 & 1.3 & 2.0 & 13.0 \\
\hline Under 35 Years ............................ & 3.4 & 1.2 & 1.2 & $\mathbf{Q}$ & $Q$ & $Q$ & 1.1 & 1.0 & $Q$ & $Q$ & $\mathbf{Q}$ & 27.3 \\
\hline 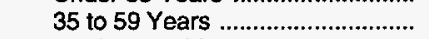 & 6.6 & 2.1 & 3.2 & 0.9 & $\mathbf{Q}$ & $\vec{Q}$ & 2.8 & 1.7 & 0.9 & $\hat{Q}$ & 0.8 & 20.8 \\
\hline 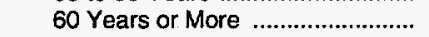 & 7.0 & 4.4 & 2.1 & $Q$ & $\bar{Q}$ & $\vec{Q}$ & 2.5 & 2.0 & 1.2 & $\bar{Q}$ & 0.8 & 16.5 \\
\hline
\end{tabular}

See footnotes at end of table. 
Table 5.17. U.S. Number of Households by Vehicle Fuel Expenditures, 1994 (Continued)

(Million Households)

\begin{tabular}{|c|c|c|c|c|c|c|c|c|c|c|c|c|}
\hline \multirow[b]{2}{*}{$\begin{array}{c}1993 \text { Household and } \\
1994 \text { Vehicle } \\
\text { Characteristics }\end{array}$} & \multirow[b]{2}{*}{$\begin{array}{c}\text { All } \\
\text { Expenditure } \\
\text { Categories }\end{array}$} & \multicolumn{5}{|c|}{$\begin{array}{l}\text { Motor Fuel Expenditures } \\
\text { (dollars per household) }\end{array}$} & \multicolumn{5}{|c|}{ Percent of Income for Motor Fuel } & \multirow[b]{3}{*}{$\begin{array}{c}\text { RSE } \\
\text { Row } \\
\text { Factor: }\end{array}$} \\
\hline & & $\begin{array}{l}\$ 500 \\
\text { or } \\
\text { Less }\end{array}$ & $\begin{array}{c}\$ 501 \\
\text { to } \\
\$ 1,000\end{array}$ & $\begin{array}{c}\$ 1,001 \\
\text { to } \\
\$ 1,500\end{array}$ & $\begin{array}{c}\$ 1,501 \\
\text { to } \\
\$ 2,000\end{array}$ & $\begin{array}{c}\$ 2,001 \\
\text { or } \\
\text { More }\end{array}$ & $\begin{array}{l}\text { Less } \\
\text { than } \\
2\end{array}$ & $\begin{array}{c}2 \\
\text { to } \\
3.9\end{array}$ & $\begin{array}{c}4 \\
\text { to } \\
5.9\end{array}$ & $\begin{array}{l}6 \\
\text { to } \\
7.9\end{array}$ & $\begin{array}{c}8 \\
\text { or } \\
\text { More }\end{array}$ & \\
\hline RSE Column Factor: & 0.5 & 1.1 & 0.9 & 1.1 & 1.2 & 1.1 & 1.0 & 0.9 & 1.1 & 1.6 & 1.2 & \\
\hline \multicolumn{13}{|l|}{ Household Composition } \\
\hline $\begin{array}{l}\text { Two or More Adults ........................... } \\
\text { Age of Householder }\end{array}$ & 35.8 & 4.7 & 10.2 & 8.6 & 6.2 & 6.1 & 10.1 & 11.1 & 6.0 & 3.6 & 5.0 & 8.6 \\
\hline 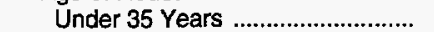 & 7.2 & 0.6 & 2.0 & 1.9 & 1.6 & 1.0 & 1.6 & 2.2 & 1.0 & 0.7 & 1.6 & 26.0 \\
\hline 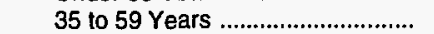 & 15.0 & 1.1 & 3.4 & 3.7 & 2.9 & 3.9 & 4.2 & 4.8 & 2.8 & 1.6 & 1.6 & 14.7 \\
\hline 60 Years or More .......................... & 13.6 & 3.0 & 4.7 & 3.0 & 1.8 & 1.2 & 4.3 & 4.0 & 2.2 & 1.3 & 1.8 & 13.6 \\
\hline \multicolumn{13}{|l|}{ Race of Householder } \\
\hline 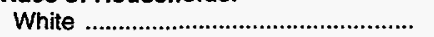 & 73.3 & 12.0 & 21.2 & 16.4 & 11.7 & 11.9 & 19.9 & 23.4 & 12.7 & 6.9 & 10.3 & 5.9 \\
\hline Black & 7.3 & 2.2 & 2.1 & 1.4 & 0.8 & 0.8 & 1.9 & 2.3 & 1.2 & $Q$ & 1.4 & 23.2 \\
\hline Other & 4.3 & 0.9 & 1.5 & 0.8 & 0.6 & $\mathbf{Q}$ & 1.4 & 0.8 & 0.8 & $a$ & $Q$ & 34.6 \\
\hline \multicolumn{13}{|l|}{ Hispanic Descent } \\
\hline 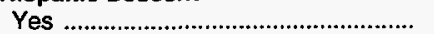 & 6.3 & 1.1 & 2.1 & 1.2 & 1.1 & 0.8 & 1.5 & 1.6 & 1.0 & 0.8 & 1.3 & 28.2 \\
\hline No & 78.6 & 14.1 & 22.7 & 17.5 & 11.9 & 12.4 & 21.7 & 24.9 & 13.6 & 7.3 & 11.2 & 5.9 \\
\hline \multicolumn{13}{|l|}{1993 family Income } \\
\hline 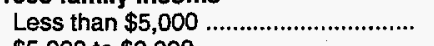 & 2.2 & Q & 1.1 & 0 & $Q$ & $Q$ & $Q$ & $Q$ & $Q$ & $Q$ & 2.0 & 35.5 \\
\hline$\$ 5,000$ to $\$ 9,999$ & 6.3 & 2.7 & 2.2 & 0.8 & $\vec{a}$ & $\vec{Q}$ & 0.7 & 0.7 & 1.0 & 1.1 & 2.9 & 24.3 \\
\hline$\$ 10,000$ to $\$ 14,999$ & 8.9 & 3.0 & 3.0 & 1.6 & 0.7 & 0.6 & 0.8 & 2.2 & 1.9 & 1.5 & 2.6 & 19.4 \\
\hline$\$ 15,000$ to $\$ 19,999$ & 8.5 & 1.9 & 3.0 & 1.7 & 1.0 & 0.8 & 1.0 & 2.2 & 2.0 & 1.3 & 2.1 & 18.8 \\
\hline 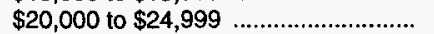 & 8.6 & 2.1 & 2.8 & 1.5 & 1.3 & 0.9 & 1.7 & 2.7 & 1.6 & 1.2 & 1.4 & 18.4 \\
\hline$\$ 25,000$ to $\$ 34,999 \ldots \ldots \ldots \ldots \ldots \ldots \ldots \ldots \ldots$ & 13.3 & 2.2 & 3.6 & 3.6 & 2.0 & 1.9 & 2.7 & 4.8 & 3.2 & 1.4 & 1.2 & 13.7 \\
\hline$\$ 35,000$ to $\$ 49,999$ & 16.5 & 1.9 & 4.3 & 4.0 & 3.0 & 3.3 & 4.9 & 6.6 & 3.5 & 1.3 & $Q$ & 12.8 \\
\hline$\$ 50,000$ to $\$ 74,999$ & 12.3 & $\mathbf{Q}$ & 3.0 & 3.4 & 2.7 & 2.7 & 5.3 & 5.5 & 1.1 & $Q$ & $\vec{Q}$ & 14.6 \\
\hline 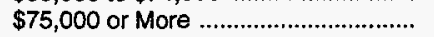 & 8.2 & $\bar{Q}$ & 1.7 & 1.9 & 1.7 & 2.6 & 6.1 & 2.0 & $\mathbf{Q}$ & $\vec{Q}$ & $\bar{Q}$ & 16.7 \\
\hline \multicolumn{13}{|l|}{ Below Poverty Line } \\
\hline 100 Percent & 9.0 & 2.5 & 3.6 & 1.3 & 1.0 & 0.6 & $\mathbf{Q}$ & 0.6 & 1.2 & 1.4 & 5.3 & 24.2 \\
\hline 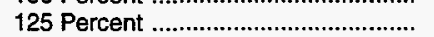 & 13.0 & 3.9 & 4.7 & 2.1 & 1.3 & 1.1 & 0.8 & 1.4 & 2.0 & 2.2 & 6.7 & 18.6 \\
\hline 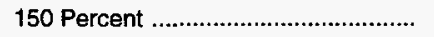 & 17.4 & 4.6 & 6.4 & 2.8 & 2.0 & 1.7 & 1.0 & 2.4 & 2.9 & 2.9 & 8.2 & 15.9 \\
\hline Eligible for Federal Assistance ${ }^{1} \ldots \ldots$ & 21.6 & 6.0 & 7.9 & 3.8 & 2.1 & 1.9 & 1.4 & 3.8 & 4.1 & 3.4 & 8.9 & 13.6 \\
\hline \multicolumn{13}{|l|}{ Number of Drivers ${ }^{2}$ (Fall 1993) } \\
\hline 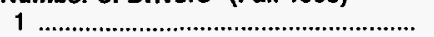 & 27.3 & 11.0 & 10.8 & 3.5 & 1.3 & 0.7 & 9.3 & 7.6 & 4.1 & 2.5 & 3.7 & 11.6 \\
\hline 2 & 45.7 & 3.3 & 12.2 & 13.5 & 9.2 & 7.6 & 12.0 & 14.9 & 8.2 & 4.3 & 6.4 & 7.8 \\
\hline 3 & 8.0 & $Q$ & 1.1 & 1.4 & 2.1 & 3.2 & 1.3 & 3.1 & 1.4 & 0.9 & 1.4 & 18.7 \\
\hline 4 or More & 2.9 & $\mathbf{Q}$ & $Q$ & $Q$ & $Q$ & 1.7 & $\mathbf{Q}$ & 0.7 & 0.8 & $Q$ & 0.6 & 27.5 \\
\hline
\end{tabular}

See footnotes at end of table. 
Table 5.17. U.S. Number of Households by Vehicle Fuel Expenditures, 1994 (Continued)

(Million Households)

\begin{tabular}{|c|c|c|c|c|c|c|c|c|c|c|c|c|}
\hline \multirow[b]{2}{*}{$\begin{array}{l}1993 \text { Household and } \\
1994 \text { Vehicle } \\
\text { Characteristics }\end{array}$} & \multirow[b]{2}{*}{$\begin{array}{c}\text { All } \\
\text { Expenditure } \\
\text { Categories }\end{array}$} & \multicolumn{5}{|c|}{$\begin{array}{l}\text { Motor Fuel Expenditures } \\
\text { (dollars per household) }\end{array}$} & \multicolumn{5}{|c|}{ Percent of Income for Motor Fuel } & \multirow[b]{3}{*}{$\begin{array}{l}\text { RSE } \\
\text { Row } \\
\text { Factor }\end{array}$} \\
\hline & & $\begin{array}{l}\$ 500 \\
\text { or } \\
\text { Less }\end{array}$ & $\begin{array}{c}\$ 501 \\
\text { to } \\
\$ 1,000\end{array}$ & $\begin{array}{c}\$ 1,001 \\
\text { to } \\
\$ 1,500\end{array}$ & $\begin{array}{c}\$ 1,501 \\
\text { to } \\
\$ 2,000\end{array}$ & $\begin{array}{c}\$ 2,001 \\
\text { or } \\
\text { More }\end{array}$ & $\begin{array}{l}\text { Less } \\
\text { than } \\
2\end{array}$ & $\begin{array}{c}2 \\
\text { to } \\
3.9\end{array}$ & $\begin{array}{c}4 \\
\text { to } \\
5.9\end{array}$ & $\begin{array}{c}6 \\
\text { to } \\
7.9\end{array}$ & $\begin{array}{c}8 \\
\text { or } \\
\text { More }\end{array}$ & \\
\hline RSE Column Factor: & 0.5 & 1.1 & 0.9 & 1.1 & 1.2 & 1.1 & 1.0 & 0.9 & 1.1 & 1.6 & 1.2 & \\
\hline \multicolumn{13}{|l|}{$\begin{array}{l}\text { Average Number of Vehicles per } \\
\text { Household During the Year }\end{array}$} \\
\hline $\begin{array}{l}\text { Part-Year Vehicle } \\
\text { Only } 1 \\
\text { Between } 1 \text { and } 2 \\
\text { Only } 2 \\
\text { Between } 2 \text { and } 3 \\
\text { Only } 3 \\
\text { Between } 3 \text { and } 4 \\
4 \text { or More }\end{array}$ & $\begin{array}{r}2.4 \\
28.6 \\
5.5 \\
28.4 \\
5.6 \\
8.4 \\
2.3 \\
3.4\end{array}$ & $\begin{array}{l}2.1 \\
11.6 \\
Q \\
0.7 \\
Q \\
Q \\
Q \\
Q\end{array}$ & $\begin{array}{l}Q \\
13.9 \\
2.4 \\
7.2 \\
Q \\
Q \\
Q \\
Q\end{array}$ & $\begin{array}{r}Q \\
2.5 \\
1.6 \\
11.4 \\
1.6 \\
1.2 \\
Q \\
Q\end{array}$ & $\begin{array}{l}Q \\
Q \\
0.8 \\
6.2 \\
1.8 \\
3.0 \\
Q \\
Q\end{array}$ & $\begin{array}{l}Q \\
Q \\
Q \\
2.9 \\
1.6 \\
3.7 \\
1.5 \\
3.0\end{array}$ & $\begin{array}{l}Q \\
11.0 \\
1.1 \\
6.8 \\
0.9 \\
1.4 \\
Q \\
Q\end{array}$ & $\begin{array}{r}Q \\
8.2 \\
1.7 \\
10.0 \\
2.1 \\
2.1 \\
0.6 \\
1.1\end{array}$ & $\begin{array}{l}Q \\
4.4 \\
1.0 \\
4.6 \\
1.2 \\
1.8 \\
0.8 \\
0.7\end{array}$ & $\begin{array}{l}\mathbf{Q} \\
2.1 \\
0.6 \\
2.9 \\
\mathbf{Q} \\
1.3 \\
\mathbf{Q} \\
\mathbf{Q}\end{array}$ & $\begin{array}{l}Q \\
3.0 \\
1.0 \\
4.1 \\
1.0 \\
1.8 \\
Q \\
0.9\end{array}$ & $\begin{array}{l}56.5 \\
10.8 \\
23.8 \\
10.6 \\
17.4 \\
16.0 \\
26.1 \\
21.4\end{array}$ \\
\hline
\end{tabular}

1 Below 150 percent of poverty line or 60 percent of median State income.

2 Approximately .5 percent of the vehicle stock was owned by households that had no drivers as of fall 1993.

$\mathrm{NF}=$ No applicable RSE row factor.

$Q=$ Data withheld either because the Relative Standard Error (RSE) was greater than 50 percent or fewer than 10 households were sampled.

Notes: - "Households with Children" category includesmembers under age 18 unless the member is the householder or spouse.- To obtain the RSE percentage for any table cell, multiply the corresponding column and row factors. Because of rounding, data may not sum to totals. $\bullet$ Data in this table are for households with vehicles for personal use. - Percentages are calculated on unrounded numbers. - See Glossary for definition of terms used in this report.

Source: Energy Information Administration, Office of Energy Markets and End Use, Form ElA-457 A of the 1993 Residential Energy Consumption Survey and Forms ElA-876 A, B, C, and D for the 1994 Residential Transportation Energy Consumption Survey. 
Table 5.18. U.S. Average Household and Vehicle Energy Expenditures, 1994

(Dollars per Household)

\begin{tabular}{|c|c|c|c|c|c|c|c|c|c|}
\hline \multirow[b]{2}{*}{$\begin{array}{l}1993 \text { Household } \\
\text { Characteristics }\end{array}$} & \multicolumn{2}{|c|}{ All Households } & \multicolumn{2}{|c|}{$\begin{array}{l}\text { Households Without } \\
\text { Vehicles }\end{array}$} & \multicolumn{4}{|c|}{ Households with Vehicles } & \multirow{3}{*}{$\begin{array}{l}\text { RSE } \\
\text { Row } \\
\text { Factor: }\end{array}$} \\
\hline & $\begin{array}{l}\text { Number } \\
\text { of } \\
\text { House- } \\
\text { holds } \\
\text { (mil- } \\
\text { lions) }\end{array}$ & $\begin{array}{l}\text { Household } \\
\text { and } \\
\text { Vehicle } \\
\text { Energy } \\
\text { Expendi- } \\
\text { tures } \\
\text { (dollars) }\end{array}$ & $\begin{array}{l}\text { Number } \\
\text { of } \\
\text { House- } \\
\text { holds } \\
\text { (mil- } \\
\text { lions) }\end{array}$ & $\begin{array}{l}\text { Household } \\
\text { Energy } \\
\text { Expendi- } \\
\text { tures } \\
\text { (dollars) }\end{array}$ & $\begin{array}{l}\text { Number } \\
\text { of } \\
\text { House- } \\
\text { holds } \\
\text { (mil- } \\
\text { tions) }\end{array}$ & $\begin{array}{l}\text { Household } \\
\text { and } \\
\text { Vehicle } \\
\text { Energy } \\
\text { Expendi- } \\
\text { tures } \\
\text { (dollars) }\end{array}$ & $\begin{array}{l}\text { Household } \\
\text { Energy } \\
\text { Expendi- } \\
\text { tures } \\
\text { (dollars) }\end{array}$ & $\begin{array}{l}\text { Motor Fuel } \\
\text { Expendi- } \\
\text { tures } \\
\text { (dollars) }\end{array}$ & \\
\hline RSE Column Factor: & t.1 & 0.7 & 2.3 & 1.1 & 1.4 & 0.6 & 0.7 & 0.9 & \\
\hline \multicolumn{10}{|l|}{ Household Characteristics } \\
\hline Total & 97.3 & 2,365 & 12.5 & 962 & 84.9 & 2,571 & 1,337 & 1,234 & 2.1 \\
\hline \multicolumn{10}{|l|}{$\begin{array}{l}\text { Energy Used in the Home-- } \\
\text { January } 1993 \text { Through December } \\
1993 \\
\text { (million Btu per household) }\end{array}$} \\
\hline 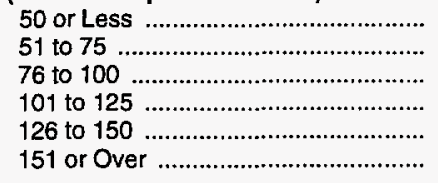 & $\begin{array}{l}20.0 \\
17.4 \\
16.0 \\
13.8 \\
10.0 \\
20.1\end{array}$ & $\begin{array}{l}1,415 \\
2,083 \\
2,362 \\
2,566 \\
2,692 \\
3,256\end{array}$ & $\begin{array}{l}4.7 \\
2.1 \\
1.8 \\
1.4 \\
0.7 \\
1.7\end{array}$ & $\begin{array}{r}569 \\
858 \\
1,019 \\
1,260 \\
1,259 \\
1,770\end{array}$ & $\begin{array}{r}15.3 \\
15.3 \\
14.2 \\
12.4 \\
9.2 \\
18.5\end{array}$ & $\begin{array}{l}1,677 \\
2,251 \\
2,536 \\
2,713 \\
2,807 \\
3,389\end{array}$ & $\begin{array}{r}715 \\
1,069 \\
1,275 \\
1,429 \\
1,530 \\
1,964\end{array}$ & $\begin{array}{r}962 \\
1,182 \\
1,260 \\
1,284 \\
1,277 \\
1,425\end{array}$ & $\begin{array}{l}4.6 \\
4.8 \\
5.0 \\
5.2 \\
5.2 \\
4.4\end{array}$ \\
\hline \multicolumn{10}{|l|}{$\begin{array}{l}\text { Expenditures for Energy Used in } \\
\text { the Home-January } 1993 \\
\text { Through December } 1993 \\
\text { (dollars per household) }\end{array}$} \\
\hline 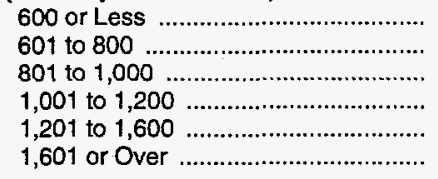 & $\begin{array}{l}10.5 \\
10.8 \\
13.4 \\
14.2 \\
23.2 \\
25.3\end{array}$ & $\begin{array}{r}998 \\
1,450 \\
1,830 \\
2,115 \\
2,554 \\
3,576\end{array}$ & $\begin{array}{l}3.4 \\
2.3 \\
2.0 \\
1.5 \\
1.8 \\
1.5\end{array}$ & $\begin{array}{r}430 \\
702 \\
910 \\
1,094 \\
1,354 \\
2,017\end{array}$ & $\begin{array}{r}7.1 \\
8.5 \\
11.4 \\
12.7 \\
21.4 \\
23.7\end{array}$ & $\begin{array}{l}1,273 \\
1,648 \\
1,989 \\
2,232 \\
2,655 \\
3,676\end{array}$ & $\begin{array}{r}453 \\
701 \\
905 \\
1,101 \\
1,392 \\
2,116\end{array}$ & $\begin{array}{r}820 \\
947 \\
1,084 \\
1,131 \\
1,263 \\
1,561\end{array}$ & $\begin{array}{l}6.4 \\
5.2 \\
5.6 \\
4.1 \\
3.2 \\
3.2\end{array}$ \\
\hline \multicolumn{10}{|l|}{$\begin{array}{l}\text { Measured Heated Area of } \\
\text { Residence (square feet) }\end{array}$} \\
\hline 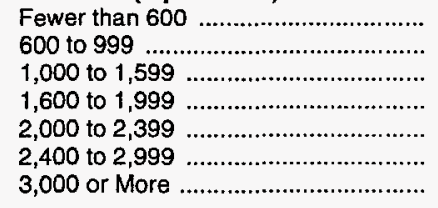 & $\begin{array}{r}8.0 \\
22.0 \\
26.9 \\
12.8 \\
9.9 \\
8.1 \\
9.8\end{array}$ & $\begin{array}{l}1,099 \\
1,730 \\
2,338 \\
2,604 \\
2,848 \\
3,224 \\
3,385\end{array}$ & $\begin{array}{l}3.5 \\
4.8 \\
2.5 \\
0.7 \\
Q \\
Q \\
0.4\end{array}$ & $\begin{array}{c}636 \\
892 \\
1,239 \\
1,361 \\
Q \\
Q \\
1,652\end{array}$ & $\begin{array}{r}4.5 \\
17.1 \\
24.3 \\
12.1 \\
9.6 \\
7.9 \\
9.4\end{array}$ & $\begin{array}{l}1,459 \\
1,965 \\
2,453 \\
2,681 \\
2,899 \\
3,256 \\
3,461\end{array}$ & $\begin{array}{r}711 \\
972 \\
1,247 \\
1,417 \\
1,576 \\
1,707 \\
1,878\end{array}$ & $\begin{array}{r}748 \\
993 \\
1,205 \\
1,264 \\
1,323 \\
1,549 \\
1,583\end{array}$ & $\begin{array}{r}10.1 \\
5.1 \\
4.0 \\
6.2 \\
5.6 \\
5.5 \\
6.8\end{array}$ \\
\hline \multicolumn{10}{|l|}{ Main Heating Fuel } \\
\hline 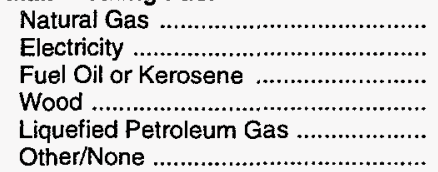 & $\begin{array}{r}52.1 \\
25.0 \\
11.6 \\
3.1 \\
4.4 \\
1.2\end{array}$ & $\begin{array}{l}2,331 \\
2,264 \\
2,528 \\
2,757 \\
2,840 \\
1,589\end{array}$ & $\begin{array}{l}7.0 \\
2.7 \\
2.0 \\
Q \\
0.4 \\
Q\end{array}$ & $\begin{array}{c}1,013 \\
763 \\
1,075 \\
Q \\
1,197 \\
Q\end{array}$ & $\begin{array}{r}45.1 \\
22.3 \\
9.6 \\
2.9 \\
4.0 \\
1.0\end{array}$ & $\begin{array}{l}2,536 \\
2,445 \\
2,828 \\
2,903 \\
3,002 \\
1,791\end{array}$ & $\begin{array}{r}1,343 \\
1,219 \\
1,594 \\
1,184 \\
1,571 \\
731\end{array}$ & $\begin{array}{l}1,193 \\
1,226 \\
1,233 \\
1,719 \\
1,431 \\
1,060\end{array}$ & $\begin{array}{r}3.2 \\
5.3 \\
5.6 \\
8.6 \\
9.1 \\
25.3\end{array}$ \\
\hline
\end{tabular}

See footnotes at end of table. 
Table 5.18. U.S. Average Household and Vehicle Energy Expenditures, 1994 (Continued)

(Dollars per Household)

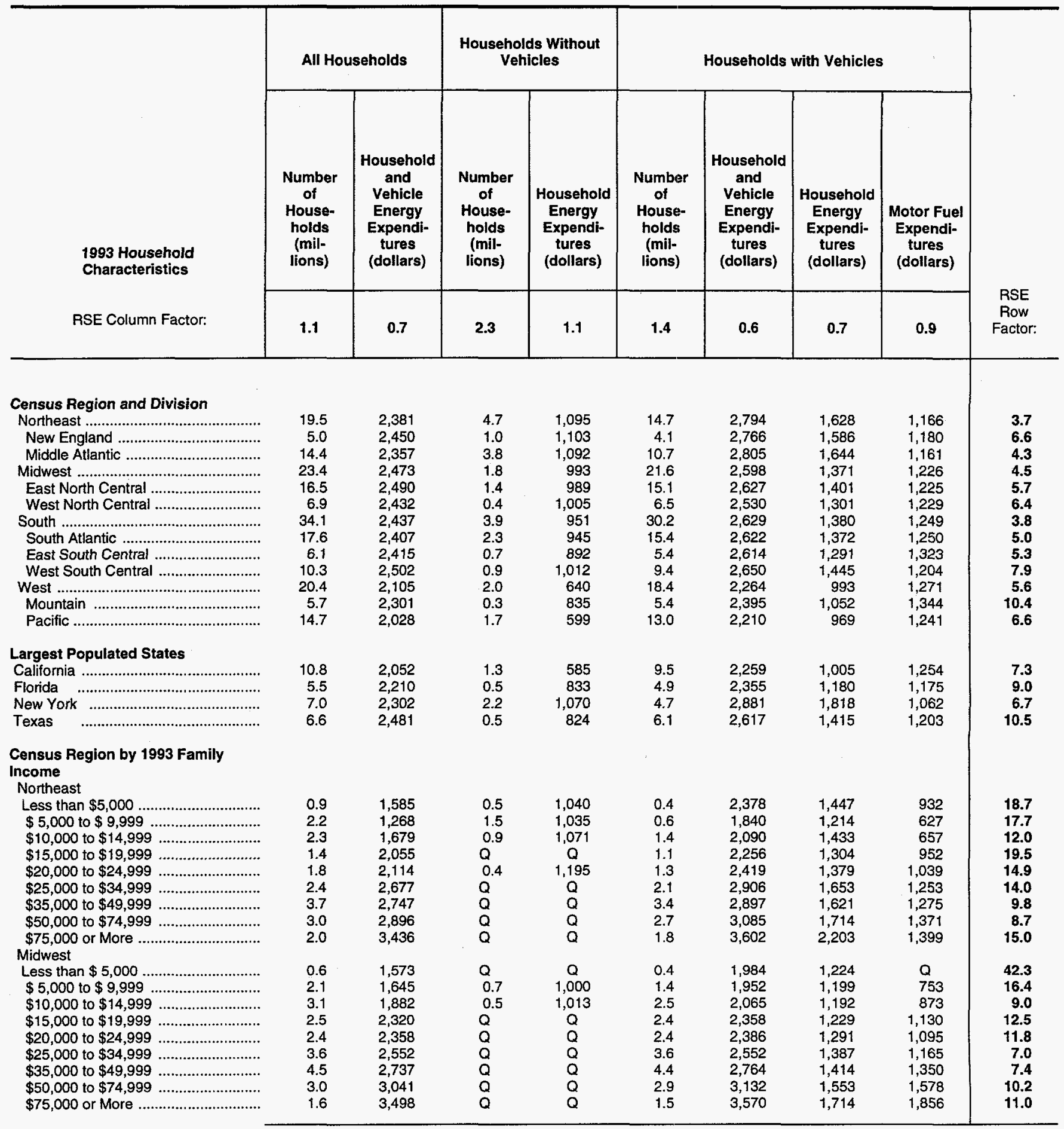

See footnotes at end of table. 
Table 5.18. U.S. Average Household and Vehicle Energy Expenditures, 1994 (Continued)

(Dollars per Household)

\begin{tabular}{|c|c|c|c|c|c|c|c|c|c|}
\hline \multirow{3}{*}{$\begin{array}{l}1993 \text { Household } \\
\text { Characteristics }\end{array}$} & \multicolumn{2}{|c|}{ All Households } & \multicolumn{2}{|c|}{$\begin{array}{c}\text { Households Without } \\
\text { Vehicles }\end{array}$} & \multicolumn{4}{|c|}{ Households with Vehicles } & \multirow{3}{*}{$\begin{array}{l}\text { RSE } \\
\text { Row } \\
\text { Factor }\end{array}$} \\
\hline & $\begin{array}{l}\text { Number } \\
\text { of } \\
\text { House- } \\
\text { holds } \\
\text { (mil- } \\
\text { lions) }\end{array}$ & $\begin{array}{l}\text { Household } \\
\text { and } \\
\text { Vehicle } \\
\text { Energy } \\
\text { Expendi- } \\
\text { tures } \\
\text { (dollars) }\end{array}$ & $\begin{array}{l}\text { Number } \\
\text { of } \\
\text { House- } \\
\text { holds } \\
\text { (mil- } \\
\text { lions) }\end{array}$ & $\begin{array}{l}\text { Household } \\
\text { Energy } \\
\text { Expendi- } \\
\text { tures } \\
\text { (dollars) }\end{array}$ & $\begin{array}{l}\text { Number } \\
\text { of } \\
\text { House- } \\
\text { holds } \\
\text { (mil- } \\
\text { lions) }\end{array}$ & $\begin{array}{l}\text { Household } \\
\text { and } \\
\text { Vehicle } \\
\text { Energy } \\
\text { Expendi- } \\
\text { tures } \\
\text { (dollars) }\end{array}$ & $\begin{array}{l}\text { Household } \\
\text { Energy } \\
\text { Expendi- } \\
\text { tures } \\
\text { (dollars) }\end{array}$ & $\begin{array}{c}\text { Motor Fuel } \\
\text { Expendi- } \\
\text { tures } \\
\text { (dollars) }\end{array}$ & \\
\hline & 1.1 & 0.7 & 2.3 & 1.1 & 1.4 & 0.6 & 0.7 & 0.9 & \\
\hline \multicolumn{10}{|l|}{ South } \\
\hline 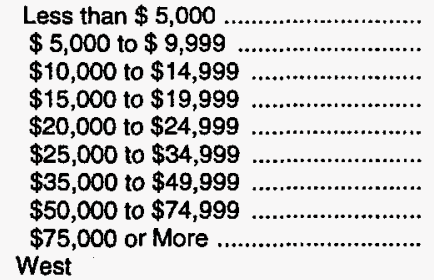 & $\begin{array}{l}1.5 \\
4.7 \\
3.6 \\
3.4 \\
3.2 \\
5.3 \\
5.6 \\
4.0 \\
2.8\end{array}$ & $\begin{array}{l}1,379 \\
1,463 \\
1,662 \\
2,289 \\
2,272 \\
2,688 \\
2,882 \\
3,203 \\
3,545\end{array}$ & $\begin{array}{l}0.7 \\
1.7 \\
0.6 \\
Q \\
Q \\
Q \\
Q \\
Q \\
Q\end{array}$ & $\begin{array}{c}1,010 \\
871 \\
832 \\
Q \\
Q \\
Q \\
Q \\
Q \\
Q\end{array}$ & $\begin{array}{l}0.8 \\
3.0 \\
3.0 \\
3.3 \\
3.0 \\
5.1 \\
5.4 \\
3.9 \\
2.7\end{array}$ & $\begin{array}{l}1,712 \\
1,798 \\
1,828 \\
2,337 \\
2,406 \\
2,735 \\
2,930 \\
3,253 \\
3,587\end{array}$ & $\begin{array}{l}1,000 \\
1,102 \\
1,081 \\
1,283 \\
1,290 \\
1,396 \\
1,433 \\
1,600 \\
1,893\end{array}$ & $\begin{array}{r}712 \\
697 \\
747 \\
1,054 \\
1,116 \\
1,339 \\
1,497 \\
1,653 \\
1,694\end{array}$ & $\begin{array}{r}19.5 \\
9.5 \\
11.8 \\
10.5 \\
13.0 \\
8.1 \\
7.5 \\
8.4 \\
11.0\end{array}$ \\
\hline 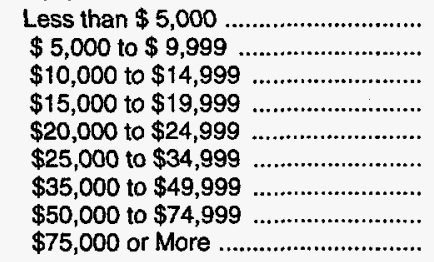 & $\begin{array}{l}0.8 \\
2.3 \\
2.4 \\
1.9 \\
2.0 \\
2.7 \\
3.3 \\
2.8 \\
2.2\end{array}$ & $\begin{array}{c}a \\
1,099 \\
1,746 \\
1,781 \\
1,918 \\
2,064 \\
2,334 \\
2,540 \\
3,298\end{array}$ & $\begin{array}{l}Q \\
1.0 \\
0.3 \\
Q \\
Q \\
Q \\
Q \\
Q \\
Q\end{array}$ & $\begin{array}{l}Q \\
557 \\
598 \\
Q \\
Q \\
Q \\
Q \\
Q \\
Q\end{array}$ & $\begin{array}{l}Q \\
1.3 \\
2.0 \\
1.7 \\
1.9 \\
2.6 \\
3.3 \\
2.7 \\
2.2\end{array}$ & $\begin{array}{c}Q \\
1,527 \\
1,931 \\
1,930 \\
1,934 \\
2,134 \\
2,334 \\
2,557 \\
3,329\end{array}$ & $\begin{array}{r}Q \\
751 \\
815 \\
822 \\
905 \\
844 \\
1,069 \\
1,105 \\
1,519\end{array}$ & $\begin{array}{c}Q \\
776 \\
1,117 \\
1,108 \\
1,029 \\
1,290 \\
1,265 \\
1,452 \\
1,810\end{array}$ & $\begin{array}{l}36.9 \\
18.0 \\
17.7 \\
16.1 \\
16.5 \\
13.3 \\
10.8 \\
10.2 \\
12.5\end{array}$ \\
\hline 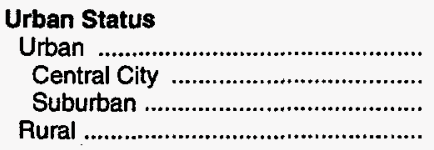 & $\begin{array}{l}76.2 \\
31.1 \\
45.1 \\
21.1\end{array}$ & $\begin{array}{l}2,308 \\
1,976 \\
2,537 \\
2,569\end{array}$ & $\begin{array}{r}10.6 \\
6.9 \\
3.7 \\
1.9\end{array}$ & $\begin{array}{r}951 \\
941 \\
970 \\
1,025\end{array}$ & $\begin{array}{l}65.6 \\
24.1 \\
41.5 \\
19.3\end{array}$ & $\begin{array}{l}2,528 \\
2,274 \\
2,675 \\
2,718\end{array}$ & $\begin{array}{l}1,330 \\
1,232 \\
1,386 \\
1,363\end{array}$ & $\begin{array}{l}1,198 \\
1,042 \\
1,289 \\
1,355\end{array}$ & $\begin{array}{l}2.6 \\
4.5 \\
3.8 \\
4.3\end{array}$ \\
\hline 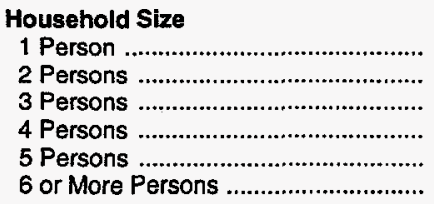 & $\begin{array}{r}23.6 \\
33.0 \\
16.2 \\
14.5 \\
7.0 \\
3.0\end{array}$ & $\begin{array}{l}1,380 \\
2,328 \\
2,778 \\
3,028 \\
3,094 \\
3,374\end{array}$ & $\begin{array}{l}6.6 \\
2.9 \\
1.2 \\
1.1 \\
0.3 \\
0.3\end{array}$ & $\begin{array}{r}804 \\
998 \\
1,198 \\
1,422 \\
1,208 \\
1,170\end{array}$ & $\begin{array}{r}17.0 \\
30.1 \\
15.0 \\
13.3 \\
6.7 \\
2.7\end{array}$ & $\begin{array}{l}1,605 \\
2,456 \\
2,905 \\
3,162 \\
3,189 \\
3,618\end{array}$ & $\begin{array}{r}949 \\
1,284 \\
1,450 \\
1,592 \\
1,618 \\
1,789\end{array}$ & $\begin{array}{r}657 \\
1,171 \\
1,455 \\
1,570 \\
1,571 \\
1,829\end{array}$ & $\begin{array}{r}4.0 \\
3.3 \\
4.8 \\
5.5 \\
8.4 \\
12.9\end{array}$ \\
\hline
\end{tabular}

See footnotes at end of table. 
Table 5.18. U.S. Average Household and Vehicle Energy Expenditures, 1994 (Continued)

(Dollars per Household)

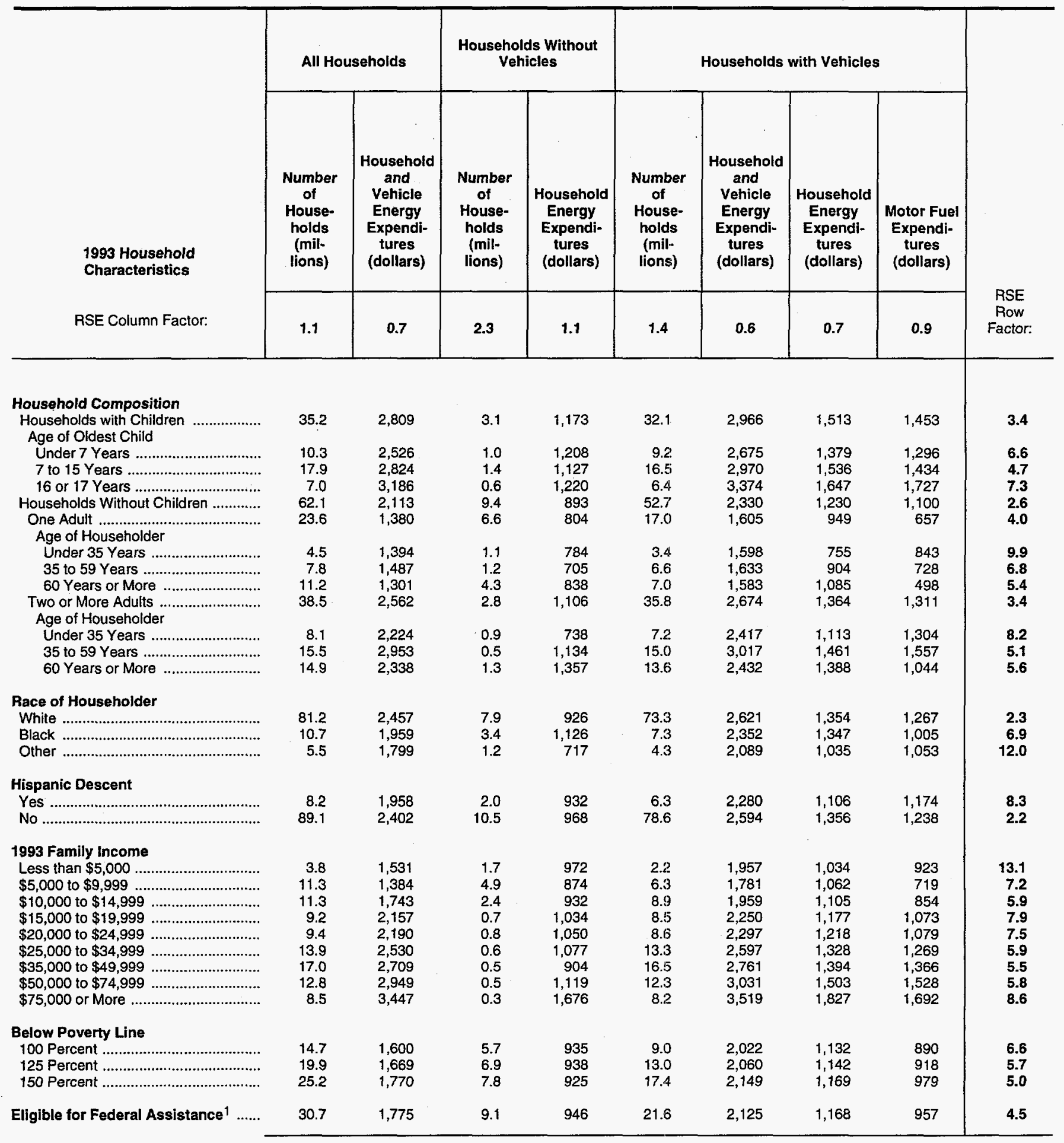

See footnotes at end of table. 
Table 5.18. U.S. Average Household and Vehicle Energy Expenditures, 1994 (Continued)

(Dollars per Household)

\begin{tabular}{|c|c|c|c|c|c|c|c|c|c|}
\hline \multirow[b]{2}{*}{$\begin{array}{l}1993 \text { Household } \\
\text { Characteristics }\end{array}$} & \multicolumn{2}{|c|}{ All Households } & \multicolumn{2}{|c|}{$\begin{array}{l}\text { Households Without } \\
\text { Vehicles }\end{array}$} & \multicolumn{4}{|c|}{ Households with Vehicles } & \multirow{3}{*}{$\begin{array}{c}\text { RSE } \\
\text { Row } \\
\text { Factor }\end{array}$} \\
\hline & $\begin{array}{l}\text { Number } \\
\text { of } \\
\text { House- } \\
\text { holds } \\
\text { (mil- } \\
\text { lions) }\end{array}$ & $\begin{array}{l}\text { Household } \\
\text { and } \\
\text { Vehicle } \\
\text { Energy } \\
\text { Expendi- } \\
\text { tures } \\
\text { (dollars) }\end{array}$ & $\begin{array}{c}\text { Number } \\
\text { of } \\
\text { House- } \\
\text { holds } \\
\text { (mil- } \\
\text { lions) }\end{array}$ & $\begin{array}{l}\text { Household } \\
\text { Energy } \\
\text { Expendi- } \\
\text { tures } \\
\text { (dollars) }\end{array}$ & $\begin{array}{c}\text { Number } \\
\text { of } \\
\text { House- } \\
\text { holds } \\
\text { (mil- } \\
\text { lions) }\end{array}$ & $\begin{array}{l}\text { Household } \\
\text { and } \\
\text { Vehicle } \\
\text { Energy } \\
\text { Expendi- } \\
\text { tures } \\
\text { (dollars) }\end{array}$ & $\begin{array}{l}\text { Household } \\
\text { Energy } \\
\text { Expendi- } \\
\text { tures } \\
\text { (dollars) }\end{array}$ & $\begin{array}{c}\text { Motor Fuel } \\
\text { Expendi- } \\
\text { tures } \\
\text { (dollars) }\end{array}$ & \\
\hline RSE Column Factor: & 1.1 & 0.7 & 2.3 & 1.1 & 1.4 & 0.6 & 0.7 & 0.9 & \\
\hline \multicolumn{10}{|l|}{ Number of Drivers ${ }^{2}$ (Fall 1993) } \\
\hline None 1 & $\begin{array}{r}11.0 \\
28.9 \\
46.3 \\
8.2 \\
3.0\end{array}$ & $\begin{array}{r}962 \\
1,709 \\
2,778 \\
3,559 \\
4,217\end{array}$ & $\begin{array}{l}10.0 \\
1.6 \\
0.5 \\
Q \\
Q\end{array}$ & $\begin{array}{c}918 \\
986 \\
1,296 \\
Q \\
Q\end{array}$ & $\begin{array}{r}1.0 \\
27.3 \\
45.7 \\
8.0 \\
2.9\end{array}$ & $\begin{array}{l}1,413 \\
1,751 \\
2,795 \\
3,604 \\
4,281\end{array}$ & $\begin{array}{r}951 \\
1,048 \\
1,422 \\
1,683 \\
1,894\end{array}$ & $\begin{array}{r}462 \\
703 \\
1,373 \\
1,921 \\
2,387\end{array}$ & $\begin{array}{r}10.9 \\
4.4 \\
3.8 \\
5.9 \\
8.9\end{array}$ \\
\hline \multicolumn{10}{|l|}{$\begin{array}{l}\text { Average Number of Vehicles per } \\
\text { Household During the Year }\end{array}$} \\
\hline 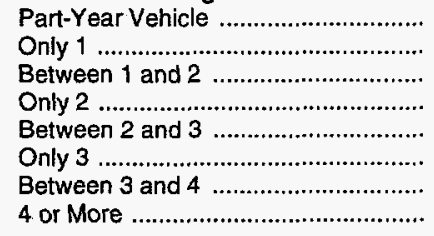 & $\begin{array}{r}2.4 \\
28.6 \\
5.5 \\
28.4 \\
5.6 \\
8.4 \\
2.3 \\
3.4\end{array}$ & $\begin{array}{l}1,434 \\
1,715 \\
2,440 \\
2,740 \\
3,262 \\
3,625 \\
4,153 \\
4,692\end{array}$ & $\begin{array}{l}Q \\
Q \\
Q \\
Q \\
Q \\
Q \\
Q \\
Q\end{array}$ & $\begin{array}{l}Q \\
Q \\
Q \\
Q \\
Q \\
Q \\
Q \\
Q\end{array}$ & $\begin{array}{r}2.4 \\
28.6 \\
5.5 \\
28.4 \\
5.6 \\
8.4 \\
2.3 \\
3.4\end{array}$ & $\begin{array}{l}1,434 \\
1,715 \\
2,440 \\
2,740 \\
3,262 \\
3,625 \\
4,153 \\
4,692\end{array}$ & $\begin{array}{l}1,110 \\
1,109 \\
1,297 \\
1,399 \\
1,514 \\
1,635 \\
1,672 \\
1,721\end{array}$ & $\begin{array}{r}324 \\
605 \\
1,142 \\
1,341 \\
1,749 \\
1,991 \\
2,481 \\
2,972\end{array}$ & $\begin{array}{r}31.3 \\
3.8 \\
7.3 \\
2.9 \\
5.0 \\
5.0 \\
9.0 \\
7.5\end{array}$ \\
\hline
\end{tabular}

1 Below 150 percent of poverty line or 60 percent of median State income.

2 Approximately 5 percent of the vehicle stock was owned by households that had no drivers as of fall 1993.

NF = No applicable RSE row factor.

$\mathrm{Q}=$ Data withheld either because the Relative Standard Error (RSE) was greater than 50 percent or fewer than 10 households were sampled.

Notes: "Households with Children" category includesmembers under age 18 unless the member is the householder or spouse.- To obtain the RSE percentage for any table cell, multiply the corresponding column and row factors. - Because of rounding, data may not sum to totals. - Data in this table are for households with vehicles for personal use. - Percentages are calculated on unrounded numbers. - See Glossary for definition of terms used in this report.

Source: Energy Information Administration, Office of Energy Markets and End Use, Form ElA-457 A of the 1993 Residential Energy Consumption Survey and Forms EIA-876 A, B, C, and D for the 1994 Residential Transportation Energy Consumption Survey. 


\section{Glossary}

Aggregate Ratio: See Mean and Ratio Estimate.

AMPD: Average miles driven per day.

Annual Vehicle-Miles Traveled: See Vehicle-Miles Traveled.

Automobile: Includes standard passenger car, 2-seater car and station wagons; excludes passenger vans, cargo vans, motor homes, pickup trucks, and sport-utility or similar vehicles. See Vehicle.

Average Household Energy Expenditures: A ratio estimate defined as the total household energy expenditures for all RTECS households divided by the total number of households. See Ratio Estimate, and Combined Household Energy Expenditures.

Average Number of Vehicles per Household: The average number of vehicles used by a household for personal transportation during 1994. For this report, the average number of vehicles per household is computed as the ratio of the total number of vehicles to the total number of households within any subgroup or "table cell." The total number of vehicles used by a household is based on the number of days each vehicle is used. For example, a total of one vehicle may represent two vehicles, each used for half of the year. See Vehicle.

Average Vehicle Fuel Consumption: A ratio estimate defined as total gallons of fuel consumed by all vehicles, divided by: (1) the total number of vehicles (for average fuel consumption per vehicle) or (2) the total number of households (for average fuel consumption per household). See Ratio Estimate.

Average Vehicle-Miles Traveled: A ratio estimate defined as total miles traveled by all vehicles, divided by: (1) the total number of vehicles (for average miles traveled per vehicle) or (2) the total number of households (for average miles traveled per household). See Ratio Estimate and Vehicle Miles Traveled.

BLS: Bureau of Labor Statistics within the U.S. Department of Labor. See Price.

British Thermal Unit (Btu): The amount of energy required to raise the temperature of 1 pound of water 1 degree Fahrenheit $(\mathrm{F})$ at or near 39.1 degrees $\mathrm{F}$ and 1 atmosphere of pressure. One Btu is about equal to the heat given off by a blue-tip match. See Conversion Factor.

Btu: See British Thermal Unit.

Btu Conversion Factor: See Conversion Factor.

Bureau of Labor Statistics (BLS) Pump Price Series: See Price.

Carburetor: A fuel delivery device for producing a proper mixture of gasoline and air, and delivering it to the intake manifold of an internal combustion engine. The efficiency of carburetors is more temperature dependent than fuel injection systems. See Fuel Injection and Diesel Fuel System. 
Census Division: A geographic area consisting of several States as defined by the U.S. Department of Commerce, Bureau of the Census. The States are grouped into nine divisions and four regions:

\section{Region}

Northeast

Midwest

South

West

\section{Division}

New England

Middle Atlantic

East North Central

West North Central

East South Central

West South Central

Mountain

Pacific

\section{States}

Connecticut, Maine, Massachusetts, New Hampshire, Rhode Island, and Vermont

New Jersey, New York, and Pennsylvania

Illinois, Indiana, Michigan, Ohio, and Wisconsin

Iowa, Kansas, Minnesota, Missouri, Nebraska, North Dakota, and South Dakota

Delaware, the District of Columbia, Florida, Georgia, Maryland, North Carolina, South Carolina, Virginia, and West Virginia

\author{
Alabama, Kentucky, \\ Mississippi, \\ and Tennessee
}

Arkansas, Louisiana, Oklahoma, and Texas

Arizona, Colorado, Idaho, Montana, Nevada, New Mexico, Utah, and Wyoming
Alaska, California, Hawaii, Oregon, and Washington

\section{Census Region: See Census Division.}

Central City: Usually one or more legally incorporated cities within the Metropolitan Statistical Area (MSA) that is significantly large by itself or large relative to the largest city in the MSA. Additional criteria for being classified "central city" include having at least 75 jobs for each 100 employed residents and having at least 40 percent of the resident workers employed within the city limits. Every MSA has at least one central city, which is usually the largest 
city. Central cities are commonly regarded as relatively large communities with a denser population and a higher concentration of economic activities than the outlying or suburban areas of the MSA. "Suburban" are those parts of the MSA that are not designated as central city. (See Metropolitan Statistical Areas, Urban, Suburban, and Rural.)

\section{Certification Files: See Environmental Protection Agency (EPA) Certification Files.}

\section{Change in Vehicle Stock: See Vehicle Acquisition and Vehicle Disposition.}

CID: Cubic Inch Displacement. See Engine Size.

Cold-Deck Imputation: A statistical procedure that replaces a missing value of an item with a constant value from an external source such as a value from a previous survey.

Combined Household Energy Expenditures: The total amount of funds spent for energy consumed in, or delivered to, a housing unit during a given period of time; and for fuel used to operate the motor vehicles that are owned or used on a regular basis by the household. For this report, expenditures for energy consumed in the housing unit are presented on an annual basis for calendar year 1993 as collected during the 1993 Residential Energy Consumption Survey. All vehicle expenditure statistics calculated for this report are on an annual basis for calendar year 1994.

The total dollar amount for energy consumed in a housing unit includes State and local taxes but excludes merchandise repairs or special service charges. Electricity expenditures and natural gas expenditures are for the amount of those energy sources consumed. Fuel oil, kerosene, and LPG expenditures are for the amount of fuel purchased, which may differ from the amount of fuel consumed.

The total dollar amount of fuel spent for vehicles is the product of fuel consumption and price. In the 1994 survey, price data were obtained from the Bureau of Labor Statistics price data and the Lundberg Survey Inc. price series. See Vehicle Fuel Expenditures, Average Household Energy Expenditures, and Price.

Conversion Factor: A number that translates units of one system into corresponding values of another system. Conversion factors are used to translate physical units of measures for various fuels into Btu equivalents. Conversion factors used in this report are:

\begin{tabular}{|c|c|}
\hline Motor Gasoline & 0.125 million Btu per gallon \\
\hline Diesel Fuel .. & 0.139 million Btu per gallon \\
\hline Propane & 0.091 million Btu per gallon \\
\hline Gasohol & 0.121 million Btu per gallon \\
\hline $\begin{array}{l}\text { Gasohol }=90 \text { percent motor } \\
1 \text { barrel }=42 \text { gallons }\end{array}$ & 10 percem etran \\
\hline
\end{tabular}

Diesel Fuel: A fuel composed of distillates obtained in petroleum refining operation or blends of such distillates with residual oil used in motor vehicles. The boiling point and specific gravity are higher for diesel fuels than for gasoline. See Diesel Fuel System.

Diesel Fuel System: Diesel engines are internal combustion engines that burn diesel oil rather than gasoline. Injectors are used to spray droplets of diesel oil into the combustion chambers, at or near the top of the compression stroke. Ignition follows due to the very high temperature of the compressed in-take air, or to the use of "glow plugs," which retain heat from previous ignitions (spark plugs are not used). Diesel engines are generally more fuel economic than gasoline engines, but must be stronger and heavier due to high compression ratios. See Diesel Fuel, Carburetor, and Fuel Injection.

Drivers: Household members who drove a vehicle on a regular basis at the time of the 1993 RECS personal interviews. 
Electricity: See Main Heating Fuel.

Energy Used in the Home: For electricity or natural gas, the quantity is the amount used by the household during the 365- or 366-day period. For fuel oil, kerosene, and liquefied petroleum gas (LPG), the quantity consists of fuel purchased, not fuel consumed. If the level of fuel in the storage tank was the same at the beginning and end of the annual period, then the quantity consumed would be the same as the quantity purchased. Information on the level of fuel in the storage tank was not included in the data collection. The time period for household consumption for energy used in the home is January 1993 through December 1993 and was collected during the 1993 Residential Energy Consumption Survey.

Engine Size: The total volume within all cylinders of an engine, when pistons are at their lowest positions. The engine is usually measured in "liters" or "cubic inches of displacement (CID)." Generally, larger engines result in greater engine power, but less fuel economy. There are 61.024 cubic inches in a liter. See Number of Cylinders.

Environmental Protection Agency (EPA) Certification Files: Computer files produced by EPA for analysis purposes. For each vehicle make, model, and year, the files contain the EPA test miles-per-gallon (mpg) values (city, highway, and 55/45 composite). These mpg values are associated with various combinations of engine and drive-train technologies (e.g., number of cylinders, engine size, gasoline or diesel fuel, and automatic or manual transmission). These files also contain information similar to that in the DOE/EPA Gas Mileage Guide, although the mpg values in that publication are adjusted for shortfall.

EPA Certification Files: See Environmental Protection Agency (EPA) Certification Files.

EPA Composite Miles per Gallon (mpg): The harmonic mean of the EPA city and highway mpg, weighted under the assumption of 55 percent city driving and 45 percent highway driving.

Family Income: The total combined annual income in 1993 of all members of the family from all sources before taxes and deductions as collected in the 1993 RECS. It includes wages, salaries, tips, commissions, income from Social Security, pensions, interest, dividends, rent, public assistance, and unemployment insurance. This includes the total income for all family members who lived in the household in 1993. Income of nonfamily members of the household is not included. "Family" includes the following types of relationships: mother, father, sister, brother, son, daughter, father-in-law, uncle, aunt, niece, grandchild, foster child, and similar relationships.

Four-Wheel Drive: See Type of Drive.

Front-Wheel Drive: See Type of Drive.

Fuel Consumption: See Vehicle Fuel Consumption.

Fuel Economy: See Miles per Gallon.

Fuel Expenditures: See Vehicle Fuel Expenditures.

Fuel Injection: A fuel delivery system whereby gasoline is pumped to one or more fuel injectors under high pressure. The fuel injectors are valves that, at the appropriate times, open to allow fuel to be sprayed or atomized into a throttle bore or into the intake manifold ports. The fuel injectors are usually solenoid operated valves under the control of the vehicle's on-board computer (thus the term "electronic fuel injection"). The efficiency of fuel-injection systems is less temperature dependent than carburetor systems. Diesel engines always use injectors. See Carburetor and Diesel Fuel Systems.

Fuel Oil: See Main Heating Fuel.

Fuel Type: See Type of Vehicle Fuel Purchased. 


\section{Full Service: See Type of Primary Service.}

\section{GPMR (Gallons per Mile Ratio): See Miles-per-Gallon (mpg) Shortfall.}

Gasohol: A fuel used in motor vehicles that is a blend of finished motor gasoline (leaded or unleaded) and alcohol (generally ethanol, but sometimes methanol), limited to 10 percent alcohol by volume. See Gasoline.

Gasoline: A complex mixture of relatively volatile hydrocarbons, with or without small quantities of additives, obtained by blending appropriate refinery streams to form a fuel suitable for use in spark ignition engines. Motor gasoline includes both leaded or unleaded grades of finished motor gasoline, blending components, and gasohol.

Hispanic Descent: This, as the question on origin, was self-determined by the respondent and is collected in the 1993 RECS. The respondent was asked, "Is the householder of Spanish or Hispanic descent?" and the respondent's answer was recorded. See Origin.

Hot-Deck Imputation: A statistical procedure for deriving a probable response to a questionnaire item concerning a household or vehicle, where no response was given during the survey. To perform the procedure, the households or vehicles are sorted by variables related to the missing item. Thus, a series of "sort categories" are formed, which are internally homogeneous with respect to the sort variables. Within each category, households or vehicles for which the questionnaire item is not missing are randomly selected to serve as "donors" to supply values for the missing item of "recipient" households or vehicles.

Household: A family, an individual, or a group of up to nine unrelated persons occupying the same housing unit at the time of the 1993 RECS interview. "Occupy" means the housing unit was the person's usual or permanent place of residence at the time of the first field contact. The household includes babies, lodgers, boarders, employed persons who live in the housing unit, and persons who usually live in the household but are away traveling or in a hospital. The household does not include persons who are normally members of the household but who were away from home as college students or members of the armed forces at the time of the contact. The household does not include persons temporarily visiting with the household if they have a place of residence elsewhere, persons who take their meals with the household but usually lodge or sleep elsewhere, domestic employees or other persons employed by the household who do not sleep in the same housing unit, or persons who are former members of the household, but have since become inmates of correction or penal institutions, mental institutions, homes for the aged or needy, homes or hospitals for the chronically ill or handicapped, nursing homes, convents or monasteries, or other places in which residents may remain for long periods of time. By definition, the number of households is the same as the number of occupied housing units. The number or households for a subgroup or table cell is estimated by summing the survey weights over all sample households in that subgroup.

Householder: The person (or one of the people) in whose name the home is owned or rented. If there is no lease or similar agreement, or if the person who owns the home or pays the rent does not live in the housing unit, the householder is the person responsible for paying the household bills, or whoever is generally in charge.

Household Composition: The configuration of the household members including number of children, number of household members, and age of household members. For this report, households were divided into households with children and households without children. Within the households with children, a further division was made depending on the age of the oldest child. Within households without children, a further division was made depending on the number of adults and then within that category, the age of the households. See Household, Householder, and Housing Unit.

Household Energy Expenditures: The total amount of funds spent for energy consumed in, or delivered to, a housing unit during a given period of time. See Combined Household Energy Expenditures.

Household Size: Number of individuals occupying a housing unit. See Household, and Housing Unit. 
Housing Unit: A structure or part of a structure where a household lives. It has direct access from the outside of the building, either directly or through a common hall. Housing units do not include group quarters such as prisons or nursing homes where 10 or more unrelated persons live. Hotel and motel rooms are considered housing units if occupied as the usual or permanent place of residence.

Imputation: A group of statistical techniques for estimating probable responses to questionnaire items concerning households or vehicles, where no responses or poor quality responses were given during the survey. The three most common techniques employed in this survey were "hot-deck," "regression," and "predictive mean matching." See HotDeck Imputation, Cold-Deck Imputation, Predictive Mean Matching, and Regression Imputation.

Intermediate-Grade Gasoline: An increasingly common grade of unleaded gasoline with an octane rating intermediate between "regular" and "premium." Octane boosters are added to gasolines to control engine pre-ignition or "knocking" by slowing combustion rates. See Regular-Grade Gasoline and Premium-Grade Gasoline.

In-Use Miles per Gallon (mpg): A mpg value that was adjusted for seasonality and annual miles traveled.

Jeep-like Vehicle: These vehicles are now referred to as sport-utility. See Sport-Utility Vehicle.

Kerosene: See Main Heating Fuel.

Large Van: See Van.

Leaded Gasoline: A fuel that contains more than 0.05 gram of lead per gallon or more than 0.005 gram of phosphorus per gallon. See Gasoline and Unleaded Gasoline.

Liquefied Petroleum Gas (LPG): See Main Heating Fuel.

Liters: See Engine Size.

Lundberg Survey Inc. Price Series: See Price.

Main Heating Fuel: The primary fuel delivered to a residential site. It may be converted to some other form of energy at the site. The following are defined as primary fuels for this report:

Electricity: Metered electric power supplied by a central utility company to a residence via underground or above-ground power lines. It does not refer to electricity generated onsite for the exclusive use of a residence. When a residence has its own generating capability, the fuel used for the generator will be specified. The Btu equivalent for electricity is the energy value of electricity as received by the household $(3,412$ Btu per kilowatthour). For this report, energy losses that occur in generating and transmitting electricity are not included in the conversion of electricity into a Btu equivalent. If these losses were to be included, the conversion rate would generally be about 10,353 Btu per kilowatthour.

Fuel Oil: No. 1, No. 2, or No. 4 grade fuel oil or residual oil that is burned for space- or water-heating purposes. No. 1 distillate fuel oil is a form of heating oil used mostly as a blending stock to insure that heavier grades of fuel flow under severe cold weather conditions. No. 2 distillate refers to both No. 2 heating oil and No. 2 diesel fuel. Although these products are not identical, they are essentially interchangeable for most applications. No. 2 fuel oil is the most common form of heating oil. No. 4 distillate is a blend of No. 2 and No. 5 or No. 6 residual fuel oil, used in large stationary diesel engines and boilers equipped with fuel preheating equipment. Residual fuel oil refers to the heavier oils that remain after the distillate fuel oils and lighter hydrocarbons are boiled off in refinery operations. 
Kerosene: The generic name for a distilled product of oil or coal, having properties similar to those of No. 1 fuel oil. Kerosene is used for cooking stoves, for space heating or water heating, or for lighting equipment that uses wicks. It is sometimes sold under the names "range oil," "stove oil," or "coal oil."

Liquefied Petroleum Gas (LPG): Any fuel gas supplied to a residence in liquid form, such as propane or butane. It is usually delivered by tank truck and stored near the residence in a tank or cylinder until used. Propane was the most common liquefied petroleum gas supplied to RECS households. Household use of LPG solely for outdoor gas grills is not considered sufficient use to mark the household as a user of LPG.

Natural Gas: Utility gas supplied by underground pipeline to individual housing units by a central utility company. It does not refer to privately owned gas wells operated by the household, nor to LPG.

Mean: The simple arithmetic average for a population; that is, the sum of all the values in a population divided by the size of the population. For this report, population means are estimated by computing the weighted sum of the sample values, then dividing by the sum of the sample weights. The mean is, thus, an aggregate ratio whose denominator is the total number of households or vehicles. See Ratio Estimate.

Measured Heated Area of Residence: The floor area of the housing unit that is enclosed from the weather and heated as collected in the 1993 RECS. Basements are included whether or not they contain finished space. Garages are included if they have a wall in common with the house. Attics that have finished space and attics that have some heated space are included. Crawl spaces are not included even if they are enclosed from the weather. Sheds and other buildings that are not attached to the house are not included. "Measured" area means that the measurement of the dimensions of the home did not rely on the respondent's reports but was an actual measurement by the interviewer using a metallic, retractable, 50-foot tape measure. "Heated area" is that portion of the measured area that is heated during most of the season. Rooms that are shut off during the heating season to save on fuel are not counted. Attached garages that are unheated and unheated areas in the attics and basements are also not counted.

\section{Metropolitan: See Urban.}

Metropolitan Statistical Area (MSA): Areas defined by the U.S. Office of Management and Budget in 1992. An MSA is (1) a county or group of contiguous counties that contain at least one city of 50,000 inhabitants or more, or (2) an urbanized area of at least 50,000 inhabitants and a total MSA population of at least 100,000 (75,000 in New England). The contiguous counties are included in an MSA if, according to certain criteria, they are essentially metropolitan in character and are socially and economically integrated with the central city. In New England, MSAs consist of towns and cities, rather than counties. (See Urban, Central City, Suburban, and Rural.)

Metropolitan Status: Refers to the geographic location of the households in relationship to MSA's. See Metropolitan, Nonmetropolitan, and Central City.

Miles per Gallon (mpg): A measure of vehicle fuel economy. Miles per gallon (mpg) as presented in this report represents "fleet miles per gallon." For each subgroup or "table cell," mpg is computed as the ratio of the total number of miles traveled by all vehicles in the subgroup to the total number of gallons consumed. For the 1994 RTECS, mpg values were assigned to each vehicle using the EPA certification files and adjusted for on-road driving.

\section{Mini-Service Pumps: See Type of Primary Service.}

Minivan: New type of small van that first appeared with that designation in 1984. Any of the smaller vans built on an automobile-type frame. Earlier models such as the Volkswagen van are now included in this category.

Model Year: As determined by the manufacturer, the model year is the year that appears in the vehicle identification number. 


\section{Motor Fuel Consumption: See Vehicle Fuel Consumption.}

Motor Fuel Expenditures: See Vehicle Fuel Expenditures.

mpg: See Miles per Gallon.

Miles-per-Gallon (mpg) Shortfall: The difference between actual on-road mpg and EPA laboratory test mpg. Milesper-gallon (mpg) shortfall is expressed as gallons-per-mile ratio (GPMR).

MSA: See Metropolitan Statistical Area (MSA).

Multistage Area Probability Sample: A sample design executed in stages with geographic "clusters" of sampling units selected at each stage. This procedure reduces survey expense while maintaining national coverage.

Natural Gas: See Main Heating Fuel.

Nonmetropolitan: Households not located within MSAs as defined by the U.S. Office of Management and Budget. See Metropolitan Statistical Area (MSA).

Number of Cylinders: In a reciprocating engine, a cylinder is the chamber in which combustion of fuel occurs and the piston moves, ultimately delivering power to the wheels. Common engine configurations include 4,6 , and 8 cylinders. Generally, the more cylinders a vehicle has, the greater the amount of engine power it has. However, more cylinders often result in less fuel economy. See Engine Size.

Number of Households: The total number of households in the United States that are represented by the sample households. In this report, most statistics are shown for the number of households with vehicles, which is a subset of the total number of households.

Number of Vehicles: See Vehicle and Vehicle Stock.

Occupied Housing Unit: A unit someone was living in as his or her usual or permanent place of residence when the first field contact was made. See Housing Unit.

On-Road Miles per Gallon (mpg): A composite mpg that was adjusted to account for the difference between the test value and the fuel economy actually obtained on the road.

Origin: The primary ethnic background of the person considered to be the householder as self-determined by the respondent. Origin of householder was collected in the 1993 RECS. Each respondent was asked, "Which of the groups on this exhibit best describes the householder?" The groups included: white, black or Negro, American Indian, Alaskan native, Asian, and Pacific Islander. The word "race" was not used in either the questionnaire or the instructions. See Hispanic Descent.

\section{Outside Central City: See Central City.}

Passenger Car: See Vehicle and Automobile.

Pickup Truck: Includes compact and full-size pickup trucks. See Vehicle.

Poverty: Low-income classifications to which certain households are assigned based on the household's annual income reported in the 1993 RECS. "Below 100 percent of poverty" encompasses a group of households with incomes below the poverty level as defined by the Bureau of the Census. "Below 125 percent of poverty" includes a group of households with incomes below 125 percent of the poverty level. These groups of the poor and near-poor represent 
alternative levels for defining poverty. The definitions of "poor" are based on the number of family members in the household and the income of the entire family.

Premium-Grade Gasoline: A grade of unleaded gasoline with a high octane rating, (approximately 92) designed to minimize preignition or engine "knocking" by slowing combustion rates. See Regular-Grade Gasoline and Intermediate-Grade Gasoline.

Predictive Mean Matching: A model-based procedure used to impute for item nonresponse. This method uses logistic models to compute predicted means that are used to statistically match each nonrespondent to a respondent with the closest predicted mean. The respondent's value is directly imputed to the nonrespondent.

Price: The dollar amount per gallon of fuel purchased. For the 1994 RTECS, fuel prices were not collected directly from the respondent. Instead fuel prices were estimated from the Bureau of Labor Statistics Retail Pump Price Survey and from the Lundberg Survey Inc. Prices

Primary Sampling Unit (PSU): A sampling unit selected at the first stage in multistage area probability sampling. A PSU typically consists of one to several contiguous counties--for example, a metropolitan area with surrounding suburban counties. The approximately 3,100 counties and independent cities of the contiguous United States were grouped into about 1,800 PSUs by a procedure similar to the one used by the Census Bureau for its Current Population Survey. PSUs can be composed of one or more MSAs or can be composed of rural counties.

\section{PSU: See Primary Sampling Unit.}

Quadrillion: The number $1,000,000,000,000,000$ or $10^{15}$.

Ratio Estimate: The ratio of two population aggregates (totals). For example, "average miles traveled per vehicle" is the ratio of total miles driven by all vehicles, over the total number of vehicles, within any subgroup or "table cell." In this report, there are two types of ratio estimates: those computed using aggregates for vehicles and those computed using aggregates for households. See Mean.

\section{Rear-Wheel Drive: See Type of Drive.}

\section{RECS: See Residential Energy Consumption Survey (RECS).}

Regression Imputation: A statistical technique for predicting the value of a numerical variable that is missing. The technique involves developing a regression equation that predicts the value of the missing variable based upon variables that are not missing or have already been imputed.

Regular-Grade Gasoline: A grade of unleaded gasoline with a lower octane rating (approximately 87) than other grades. Octane boosters are added to gasoline to control engine preignition or "knocking" by slowing combustion rates. See Intermediate-Grade Gasoline and Premium-Grade Gasoline.

\section{Relative Standard Error: See RSE (Relative Standard Error).}

Residential: Occupied housing units, including mobile homes, single-family housing units (attached and detached), and apartments. The definition of "occupied housing units" is the same as that used by the U.S. Bureau of the Census. See Household and Housing Unit.

Residential Energy Consumption Survey (RECS): A national multistage probability sample survey conducted by the Energy End Use Division of the Energy Information Administration. The RECS provides baseline information on how households in the United States use energy. The RTECS sample is a subset of the RECS. Household demographic characteristics reported in the RTECS publication are collected during the RECS personal interview. 
RSE (Relative Standard Error): A measure of the reliability or precision of a survey statistic. Variability occurs in survey statistics because the different samples that could be drawn would each produce different values for the survey statistics. The RSE is a measure of precision on a percentage scale. The RSE is defined as the standard error of a survey estimate, divided by the survey estimate and multiplied by 100. (Standard error is the square root of the variance.) For example, an RSE of 50 percent means that the standard error is half as large as the survey estimate.

RSE Column Factor: An adjustment factor that appears above each column of the tables and is used to compute RSEs. For a survey estimate in a particular row and column of a table (that is, a particular "cell"), the approximate RSE is obtained by multiplying the RSE row factor by the RSE column factor for that cell. See RSE and RSE Row Factor.

RSE Row Factor: A factor that appears to the right of each row of the tables, and is used to compute RSE's. For a survey estimate in a particular row and column of a table (that is, a particular "cell"), the approximate RSE is obtained by multiplying the RSE row factor by the RSE column factor for that particular cell. The row factor is equal to the geometric mean of the RSE's in a particular row of the tables. See RSE and RSE Column Factor

Rural: Households not located within Metropolitan Statistical Areas as defined by the U.S. Office of Management and Budget for 1993. See Metropolitan Statistical Area and Urban Status.

Sampling: The procedure used to select housing units for interview from the population of residential housing units in the United States. See Multistage Area Probability Sample.

Self-Service or Mini-Service: See Type of Primary Service.

Shortfall: See Miles-per-Gallon (mpg) Shortfall.

Sport-Utility Vehicle: Includes light trucks that are similar to jeeps. Other common terms for these vehicles are sportutility, special purpose, utility or off-the-road vehicles. They may have a four- or two-wheel drive. See Vehicle.

Suburban: Those parts of the MSA that are not designated as central city. Suburban areas are referred to as "outside central city." See Metropolitan Statistical Area.

Transmission Type: The householder was asked if each vehicle had an automatic or manual shift transmission. The transmission is the part of a vehicle that transmits motive force from the engine to the wheels, usually by means of gears for different speeds using either a hydraulic "torque-converter" (automatic) or clutch assembly (manual). On front wheel drive cars, the transmission is often called a "transaxle." Fuel economy is usually higher with manual transmissions than automatic transmissions, although newer automatic transmissions are narrowing the difference.

Transportation Energy Expenditures: See Vehicle Fuel Expenditures and Combined Household Energy Expenditures.

Type of Drive: Refers to which wheels the engine power is delivered to, the so-called "drive wheels." Rear-wheel drive, has drive wheels on the rear of the vehicle. Front-wheel drive, a newer technology, has drive wheels on the front of the vehicle. Four-wheel drive uses all four wheels as drive wheels, and is found mostly on sport-utility vehicles and trucks, though it is becoming increasingly more common on station wagons and vans.

Type of Fuel System: See Carburetor, Fuel-Injection, and Diesel Fuel Systems.

Type of Vehicle Fuel Purchased: The predominant type of fuel purchased during 1994. Data categories are leaded and unleaded gasoline, diesel motor fuel, and "other" which includes propane and gasohol. See Gasoline, Gasohol, Unleaded Gasoline, Leaded Gasoline, Regular-Grade Gasoline, Intermediate-Grade Gasoline, and PremiumGrade Gasoline. 
Type of Primary Service: The dominant type of service the respondent uses at the service station. Response categories include "full-service pumps," "self- or mini-service pumps," or "both equally." Mini-service is provided when attendants pump the vehicle fuel but do not provide any other service, such as checking the tire pressure.

Urban: Urban refers to a group of households located within Metropolitan Statistical Areas (MSAs) as defined by the U.S. Office of Management and Budget in 1993. For this report, urban is composed of central city and suburban areas. An MSA is (1) a county or group of contiguous counties that contain at least one city of 50,000 inhabitants or more, or (2) an urbanized area of at least 50,000 inhabitants and a total MSA population of at least 100,000 (75,000 in New England). The contiguous counties are included in an MSA if, according to certain criteria, they are essentially metropolitan in character and are socially and economically integrated with the central city. In New England, MSAs consist of towns and cities rather than counties. (See Central City, Suburban, and Rural.)

Unleaded Gasoline: Contains not more than 0.05 gram of lead per gallon and not more than 0.005 gram of phosphorus per gallon. Premium, regular and intermediate grades are included, depending on the octane rating. See Gasoline, Leaded Gasoline, Regular-Grade Gasoline, Intermediate-Grade Gasoline, and Premium-Grade Gasoline.

Van: Includes large vans. Generally, the distinction between large vans and minivans is made by the respondents' answers to "Type of Vehicle" question. Exceptions were: (1) Volkswagen vans were categorized as minivans, and (2) all other pre-1983 vans were categorized as vans.

Vehicle: For the RTECS, vehicles were any motorized vehicles used by U.S. households for personal transportation. Excluded were: motorcycles, mopeds, large trucks, and buses. Included were: automobiles, station wagons, passenger vans, cargo vans, motor homes, pickup trucks, and sport-utility or similar vehicles. In order to be included, vehicles must be: (1) owned by members of the household; (2) company cars not owned by household members but regularly available to household members for their personal use and are ordinarily kept at home; or (3) rented or leased for 1 month or more. See Vehicle Stock, Vehicles Used on the Job, Automobile, Minivans, Vans, Pickup Trucks, and Sport-Utility Vehicles.

Vehicle Acquisition: The number of vehicles a household acquires or obtains during the RTECS survey year. The average number of vehicles in the stock is computed using these data. See Vehicle Disposition.

Vehicle Disposition: The number of vehicles a household disposes of during the RTECS survey year. Disposed vehicles include those sold, traded, or the owner moved out of the household. The average number of vehicles in the stock is computed using these data. See Vehicle Acquisition.

Vehicle Fuel Consumption: Vehicle fuel consumption is computed as the vehicle-miles traveled divided by the fuel economy reported in miles per gallon (mpg). For the 1994 RTECS, vehicle fuel consumption was derived from the actual vehicle mileage collected in the RTECS and the assigned mpg values were obtained from the EPA certification files and adjusted for on-road driving.

Vehicle Fuel Economy: See Miles per Gallon (mpg).

Vehicle Fuel Expenditures: The cost, including taxes, of the gasoline, gasohol or diesel fuel added to the vehicle's tank. Expenditures do not include the cost of oil or other items that may have been purchased at the same time as the vehicle fuel.

Vehicle Identification Number (VIN): A set of codes, usually alpha-numeric characters, assigned to a vehicle at the factory and inscribed on the vehicle. When decoded, the VIN provides vehicle characteristics. The VIN was used in the 1994 RTECS to help match vehicles to the EPA certification file for calculating miles-per-gallon values. See Environmental Protection Agency (EPA) Certification Files. 
Vehicle-Miles Traveled (VMT): The number of miles traveled nationally by the RTECS vehicles for a period of 1 year. In the RTECS, VMT were either calculated using two odometer readings or, for vehicles with less than two odometer readings, imputed using a regression estimate. See Average Vehicle-Miles Traveled.

Vehicle Stock: The number of vehicles owned or used by a household for personal transportation. In the RTECS, with the exception of the statistics reported as of July 1994, a vehicle was defined in terms of a "vehicle year." If a vehicle was present in a household for the entire year, it was counted as one vehicle. If a vehicle was present in a household for one-half of the year, it was counted as only one-half of a vehicle. Therefore, the number of vehicles a sample household was considered as having during the survey year was computed as the days of possession summed over all vehicles in the household, divided by 366 days (1994 was a leap year). See Average Number of Vehicles and Vehicles.

Vehicle Used on the Job: A vehicle used by anyone in the household for job-related activities, excluding commuting to and from work. These vehicles are included in the RTECS. See Vehicle.

VIN: See Vehicle Identification Number.

VMT: See Vehicle-Miles Traveled. 


\section{Publication Order Form}

\section{Household Vehicles Energy Consumption 1994}

Published: August 1997

Energy Information Administration

GPO Stock No. 061-003-01001-6

Price per copy: $\$ 10.00$

Personal Name or Attention Line:

Company Name, if applicable:

Street Address:

City, State, Zip Code:

Daytime Phone Number (area code first):

Purchase Order No:

May we make your name and address available to other mailers? yes no

Household Vehicles Energy Consumption 1994 presents statistics about energy-related characteristics of highway vehicles available for personal use by members of U.S. households. The data were collected in the 1994 Residential Transportation Energy Consumption Survey and covered household and vehicle characteristics, vehicle-miles traveled, and vehicle-fuel consumption and costs.

Please include payment with this order form. Allow a minimum of 4 weeks for domestic delivery and an additional 6 weeks for international delivery.

Quantity

$$
\mathrm{X} \$ 10.00=\$
$$
(total due). (International customers add 25\%)

Check payable to Superintendent of Documents

GPO Deposit Account No.

VISA or MasterCard Account No.

Authorizing Signature Credit Card Expiration Date

Note: Price includes regular domestic postage and handling. It is subject to change.

Mail order form to: U.S. Government Printing Office

P.O. Box 371954

Pittsburgh, PA 15250-7954

Or fax order form to: $202-512-2250$ 
